RODRIGO JULIANI CORREA DE GODOY

\title{
PLANTWIDE CONTROL: A REVIEW AND PROPOSAL OF AN AUGMENTED HIERARCHICAL PLANTWIDE CONTROL DESIGN TECHNIQUE
}

Thesis presented to the Polytechnic School of the University of São Paulo to acquire the title of Doctor of Science

Tese apresentada à Escola Politécnica da Universidade de São Paulo para obtenção do título de Doutor em Ciências 


\section{PLANTWIDE CONTROL: A REVIEW AND PROPOSAL OF AN AUGMENTED HIERARCHICAL PLANTWIDE CONTROL DESIGN TECHNIQUE}

Thesis presented to the Polytechnic School of the University of São Paulo to acquire the title of Doctor of Science

Concentration area:

System Engineering

Supervisor:

Prof. Dr. Claudio Garcia
Tese apresentada à Escola Politécnica da Universidade de São Paulo para obtenção do título de Doutor em Ciências

Área de Concentração: Engenharia de Sistemas

Orientador:

Prof. Dr. Claudio Garcia 
Este exemplar foi revisado e corrigido em relação à versão original, sob responsabilidade única do autor e com a anuência de seu orientador.

São Paulo, de de

Assinatura do autor:

Assinatura do orientador:

Catalogação-na-publicação

\section{Godoy, Rodrigo Juliani Correa de}

Plantwide Control: A Review and Proposal of an Augmented Hierarchical Plantwide Control Design Technique / R. J. C. Godoy -- versão corr. -- São Paulo, 2017.

$198 \mathrm{p}$.

Tese (Doutorado) - Escola Politécnica da Universidade de São Paulo. Departamento de Engenharia de Telecomunicações e Controle.

1.Controle Plantwide 2.Controle (Teoria de sistemas e controle) 3.Controle de Processos 4.Sistemas de Controle 5.Controle Automático I.Universidade de São Paulo. Escola Politécnica. Departamento de Engenharia de Telecomunicações e Controle Il.t. 


\section{AGRADECIMENTOS (In Portuguese)}

Primeiramente gostaria de agradecer a todos que contribuíram, direta ou indiretamente, com a realização deste trabalho.

Ao orientador e amigo, Professor Dr. Claudio Garcia, por todas as suas sugestões e contribuições para o desenvolvimento e melhorias do trabalho. Também agradeço pelos inúmeros ensinamentos e oportunidades oferecidos nestes dez anos em que trabalhamos juntos em diversas frentes.

À Fernanda, pelo contínuo apoio e companheirismo, pelo interesse nos meus trabalhos e pelas diversas sugestões que ajudaram na conclusão desta tese.

À minha família, pelo apoio e incentivo à realização deste trabalho.

Finalmente, a todos os amigos e colegas que incentivaram a realização deste estudo e meus trabalhos em pesquisa e desenvolvimento. 
"My time here is ended. Take what I have taught you and use it well." 


\begin{abstract}
The problem of designing control systems for entire plants is studied. A review of previous works, available techniques and current research challenges is presented, followed by the description of some theoretical tools to improve plantwide control, including the proposal of an augmented lexicographic multi-objective optimization procedure. With these, an augmented hierarchical plantwide control design technique and an optimal multi-objective technique for integrated control structure selection and controller tuning are proposed. The main contributions of these proposed techniques are the inclusion of system identification and optimal control tuning as part of the plantwide design procedure for improved results, support to multi-objective control specifications and support to any type of plant and controllers. Finally, the proposed techniques are applied to industrial benchmarks to demonstrate and validate its applicability.
\end{abstract}

Keywords: Plantwide control, control structure selection, control design, control tuning, optimal control tuning, multi-objective optimization, system identification, optimal control. 


\section{RESUMO}

O problema de projetar sistemas de controle para plantas inteiras é estudado. Uma revisão de trabalhos anteriores, técnicas disponíveis e atuais desafios de pesquisa é apresentada, seguida da descrição de algumas ferramentas teóricas para melhorar o controle plantwide, incluindo a proposta de um procedimento de otimização multi-objetivo lexicográfico aumentado. Com tais elementos, são propostas uma nova técnica hierárquica aumentada de projeto de sistemas de controle plantwide e uma técnica multi-objetivo para seleção de estrutura de controlador integrada à sintonia ótima do controlador. As principais contribuições das técnicas propostas são a inclusão de identificação de sistemas e sintonia ótima de controladores como parte do procedimento de projeto de controle plantwide para melhores resultados, suporte a especificações multi-objetivo e suporte a quaisquer tipos de plantas e controladores. Finalmente, as técnicas propostas são aplicadas a benchmarks industriais para demonstrar e validar sua aplicabilidade.

Palavras-chave: Controle plantwide, seleção de estrutura de controle, projeto de sistemas de controle, sintonia de controladores, sintonia ótima de controladores, otimização multi-objetivo, identificação de sistemas, controle ótimo. 


\section{LIST OF FIGURES}

Figure 1.1 - Number of Plantwide Control articles published during the period 19902010 (Rangaiah \& Kariwala, 2012).

Figure 2.1 - Classification of plantwide control architectures (Ochoa, et al., 2010). .10

Figure 2.2 - Typical hierarchical control (Skogestad, 2000a)..............................11

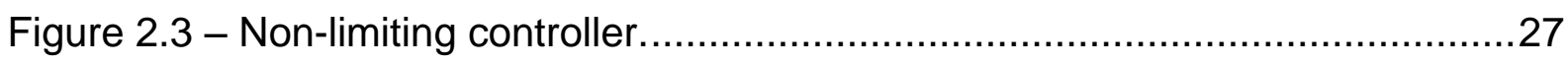

Figure 3.1 - A simple process with recycle. ..............................................36

Figure 3.2 - A simple process with multiple controllers. ....................................40

Figure 3.3 - Goal programming publications in the period 1975-2008 (Jones \& Tamiz, 2010).

Figure 3.4 - Augmented Lexicographic Multi-Objective Optimization. .46

Figure 3.5 - Pareto front. 47

Figure 3.6 - Optimal Control Tuner (Juliani, 2012) ........................................51

Figure 5.1 - Wood and Berry distillation column (Wood \& Berry, 1973).................66

Figure 5.2 - Results for the Wood and Berry Distillation Column - Process Outputs.

Figure 5.3 - Results for the Wood and Berry Distillation Column - Process Inputs. .67 Figure 5.4 - III-Conditioned High Purity Distillation Column (Skogestad, et al., 1988).

Figure 5.5 - Results for the High Purity Distillation Column - Process Outputs........70

Figure 5.6 - Results for the High Purity Distillation Column - Process Inputs...........71

Figure 5.7 - Binary Distillation Column (Luyben, 1989). ......................................72

Figure 5.8 - Results for the Binary Column for PID controllers with T = $60 \mathrm{~s}$ - Process Outputs.

Figure 5.9 - Results for the Binary Column for PID controllers with $\mathrm{T}=60 \mathrm{~s}-$ Process Inputs.

Figure 5.10 - Results for the Binary Column for PID controllers with $T=1 \mathrm{~s}-$ Process Outputs......

Figure 5.11 - Results for the Binary Column for PID controllers with $\mathrm{T}=1 \mathrm{~s}-$ Process Inputs. .74

Figure 5.12 - Results for the Industrial Furnace - Process Outputs. .77

Figure 5.13 - Results for the Industrial Furnace - Process Inputs. .77 
Figure $5.14-3 \times 3$ binary distillation column (Ogunnaike, et al., 1983) ....................79

Figure 5.15 - Results for the $3 \times 3$ Distillation Column - Process Outputs. ...............81

Figure 5.16 - Results for the $3 \times 3$ Distillation Column - Process Inputs. ..................81

Figure 5.17 - Shell HOF and control problem (Zheng, et al., 1994).......................87

Figure 5.18 - PID control. Configurations 1 (red) and 2 (blue).............................90

Figure 5.19 - Identified model for the HOF Plant in closed loop with PID controllers.

Figure 5.20 - Results for the Shell HOF Benchmark............................................94

Figure 5.21 - FCC benchmark (Grosdidier, et al., 1993)....................................96

Figure 5.22 - PID Control for the FCC Benchmark. ............................................ 99

Figure 5.23 - Identified model for the FCC Plant in closed loop with PID controllers. Process (grey) and model (blue) responses.....................................100

Figure 5.24 - Results for the FCC Benchmark.................................................102

Figure 6.1 - The Tennessee Eastman Challenge Process. .................................107

Figure 6.2 - Tennessee Eastman Challenge Model Implementation.....................111

Figure 6.3 - Tennessee Eastman with stabilizing control..................................118

Figure 6.4 - Tennessee Eastman Challenge with regulatory control. ....................124

Figure 6.5 - Tennessee Eastman Challenge with regulatory and supervisory control

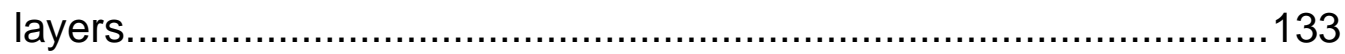

Figure 6.6 - Step responses for the feed flow controllers. .................................135

Figure 6.7 - Feed flow regulatory responses. ................................................136

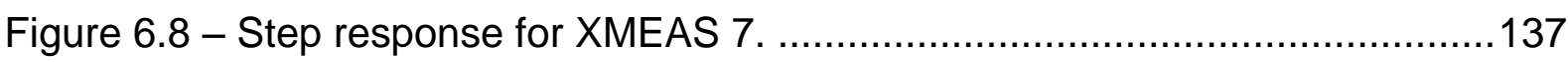

Figure 6.9 - Regulatory response for XMEAS 7 and XMEAS 10 ......................137

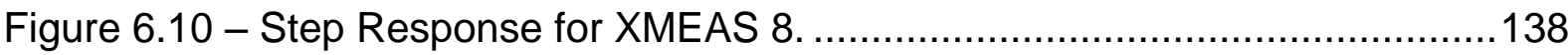

Figure 6.11 - Regulatory response for XMEAS 8 and XMEAS 11 .....................139

Figure 6.12 - Step response for XMEAS 9. ..................................................140

Figure 6.13 - Regulatory Response for XMEAS 9. .......................................140

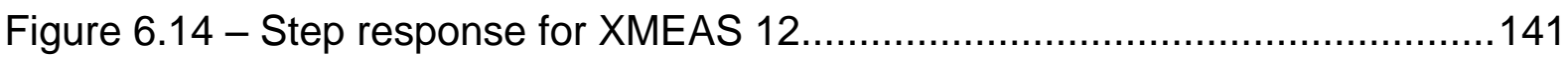

Figure 6.15 - Regulatory response for XMEAS 12 and XMEAS 14 .....................141

Figure 6.16 - Step response for XMEAS 15 ...................................................... 142

Figure 6.17 - Regulatory response for XMEAS 15 and XMEAS 17 ....................142

Figure 6.18 - Step response for XMEAS 18 .................................................143

Figure 6.19 - Regulatory response for XMEAS 18 and XMEAS 19...................143 


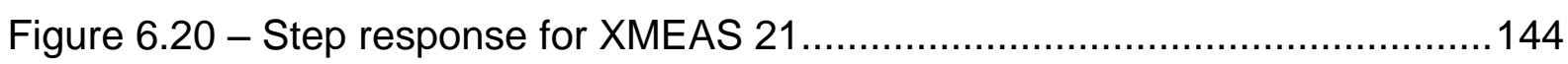

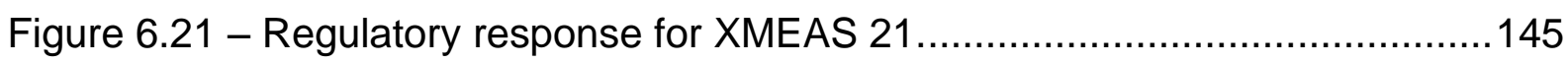

Figure 6.22 - Process variables for the Tennessee Eastman in nominal operation.

Figure 6.23 - Manipulated variables for the Tennessee Eastman in nominal operation.

Figure 6.24 - Process variables for the Tennessee Eastman in mode 1 operation. 148 Figure 6.25 - Manipulated variables for the Tennessee Eastman in mode 1 operation. 149

Figure 6.26 - Process variables for the Tennessee Eastman in mode 3 operation....... 149

Figure 6.27 - Manipulated variables for the Tennessee Eastman in mode 3 operation. 150

Figure 6.28 - Operating costs for the Tennessee Eastman Challenge Process. ....150

Figure A.1 - Original dataset..................................................................171

Figure A.2 - Filtered and normalized dataset.................................................172

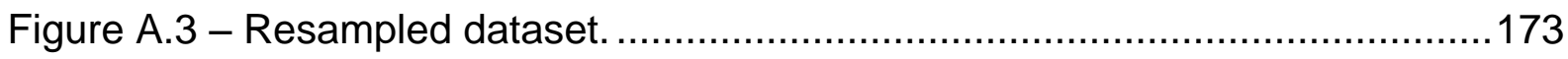

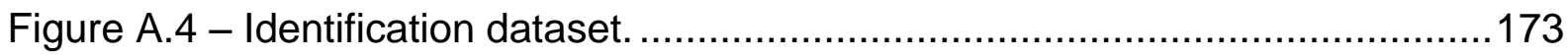

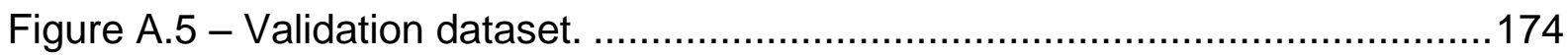

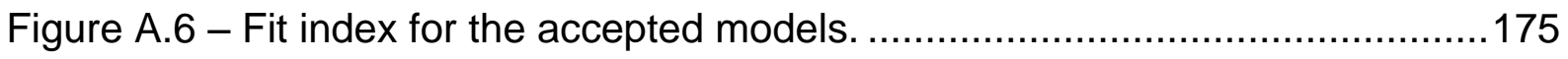

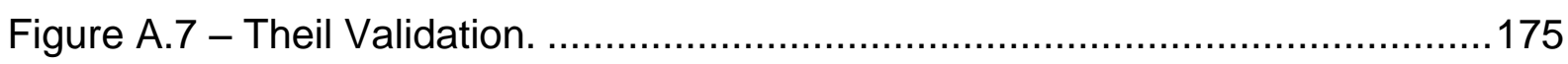

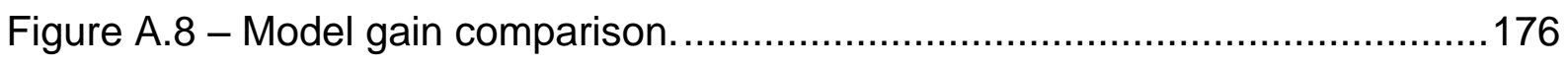

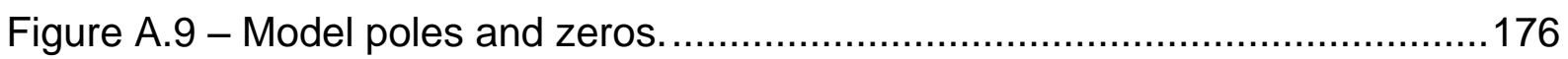

Figure A.10 - Final model performance for the validation dataset. .......................177

Figure A.11 - Final model performance for the identification dataset.....................177 


\section{LIST OF TABLES}

Table 2.1 - Plantwide control design techniques proposed between 2000 and 2009 (Vasudevan, et al., 2009). .13

Table 2.2 - Qualifications of the described plantwide control design techniques......20 Table 2.3 - Benchmarks for Plantwide Control (Vasudevan, et al., 2009), (updated and reorganized).

Table 5.1 - Optimal PID Tuning Parameters for the Wood and Berry Benchmark...67

Table 5.2 - Performance Indicators for the Wood and Berry Benchmark .68

Table 5.3 - Optimal PID Tuning Parameters for the Wood and Berry Benchmark....70 Table 5.4 - Performance Indicators for the High Purity Distillation Column Benchmark

Table 5.5 - Optimal PID Tuning Parameters for the Binary Distillation Column Benchmark .73

Table 5.6 - Performance Indicators for the Binary Distillation Column Benchmark...75 Table 5.7 - Optimal PID Tuning Parameters for the Industrial Furnace Benchmark.76 Table 5.8 - Performance Indicators for the Industrial Furnace Benchmark. .78

Table 5.9 - Optimal PID Tuning Parameters for the 3x3 Distillation Column Benchmark

Table 5.10 - Performance Indicators for the 3x3 Distillation Column Benchmark [1] 82 Table 5.11 - Performance Indicators for the 3x3 Distillation Column Benchmark [2] 83

Table 5.12 - Variable constraints for the Shell HOF (Zheng, et al., 1994). 89

Table 5.13 - Optimal PID Tuning Parameters for the Shell HOF Benchmark .90

Table 5.14 - Performance Indicators for the Regulatory Control of the Shell HOF Benchmark.

Table 5.15 - Optimal MPC Tuning Parameters for the Shell HOF Benchmark .93

Table 5.16 - SSE Performance Indexes for the Shell HOF.

Table 5.17 - Variable constraints for the FCC benchmark (Grosdidier, et al., 1993).97

Table 5.18 - Optimal PID Tuning Parameters for the FCC Benchmark .99

Table 5.19 - Optimal MPC Tuning Parameters for the FCC Benchmark 101

Table 5.20 - Performance Indicators for the FCC Benchmark [1] 102

Table 5.21 - Performance Indicators for the FCC Benchmark [2] 103

Table 5.22 - SSE Performance Indexes for the FCC Benchmark. 104 
Table 6.1 - Tennessee Eastman Process Manipulated Variables (Downs \& Vogel, 1993). 107

Table 6.2 - Tennessee Eastman Process Continuous Measured Variables (Downs \& Vogel, 1993). 108

Table 6.3 - Tennessee Eastman Process Sampled Analytical Variables (Downs \& Vogel, 1993) 109

Table 6.4 - Tennessee Eastman Process Operating Constraints (Downs \& Vogel, 1993).

Table 6.5 - I/O effect matrix for unstable variables in open loop.

Table 6.6 - I/O effect matrix for the stabilized plant. 119

Table 6.7 - I/O effect matrix for the stabilized plant. 121

Table 6.8 - I/O Pairings and PID tunings $(T s=1 s)$ for the regulatory control layer. 123

Table 6.9 - I/O effect matrix for the process with active regulatory controllers. 127

Table 6.10 - Scale factors for the MPC manipulated variables. 129

Table 6.11 - Scale factors for the output variables used in the MPC controller. .....130

Table 6.12 - MPC constraints for the controlled variables. 131

Table 6.13 - Constraints for the MPC manipulated variables 132

Table 6.14 - Setpoints for the three tested scenarios. 146

Table 6.15 - Average operating cost for $72 \mathrm{~h}$ of operation in three operating modes. 


\section{LIST OF EQUATIONS}

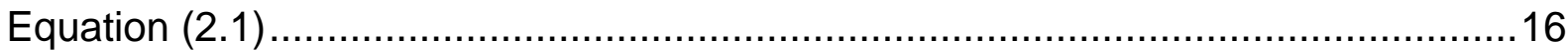

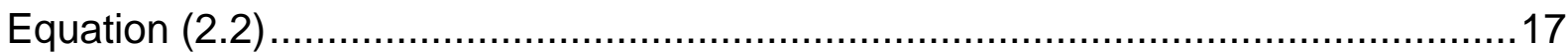

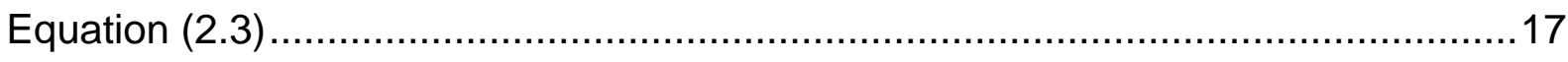

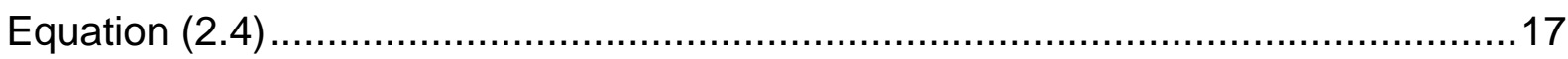

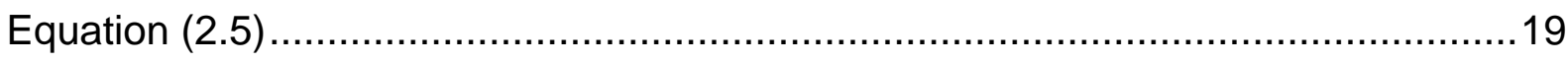

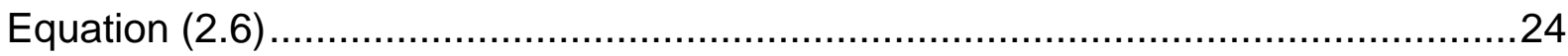

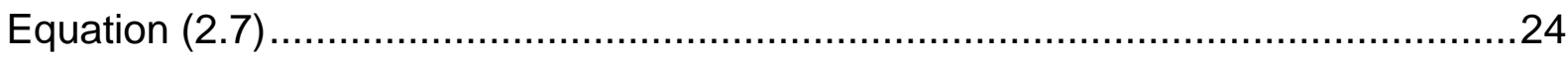

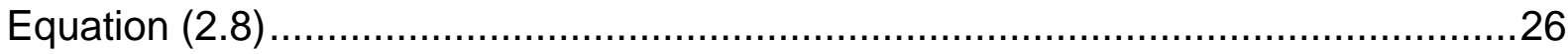

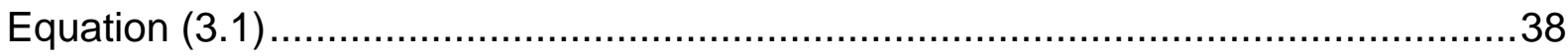

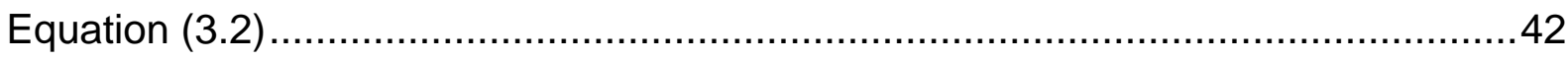

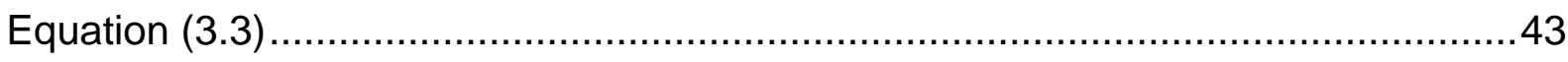

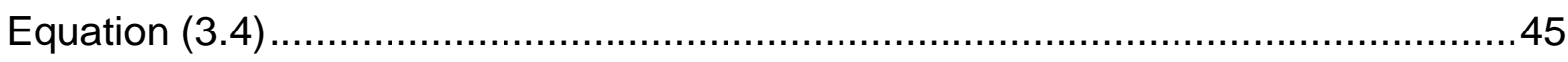

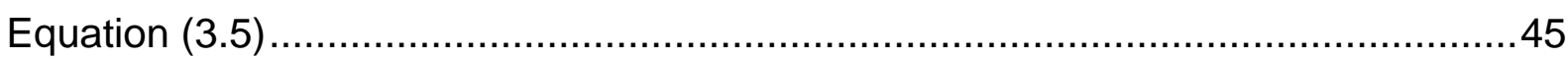

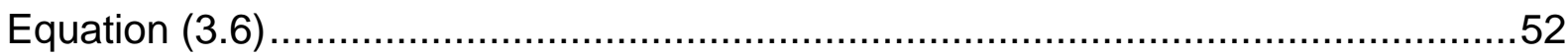

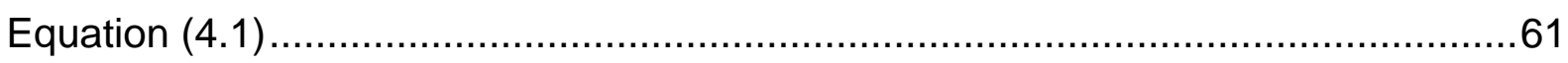

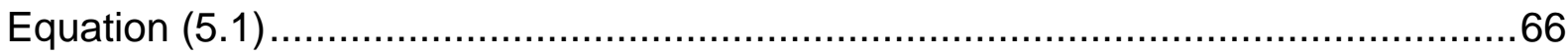

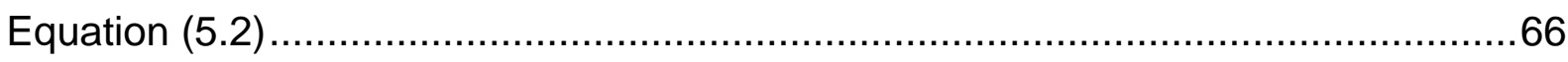

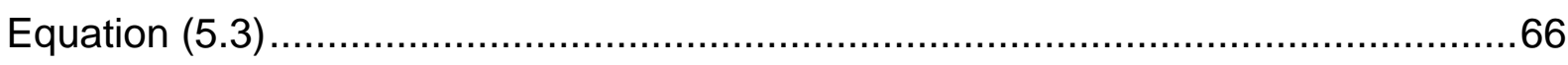

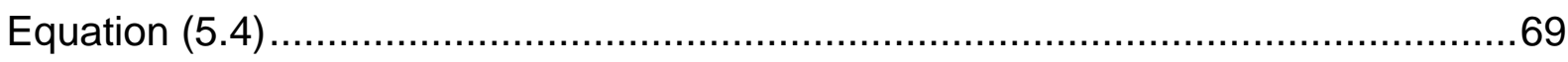

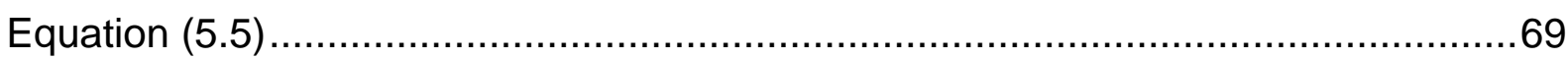

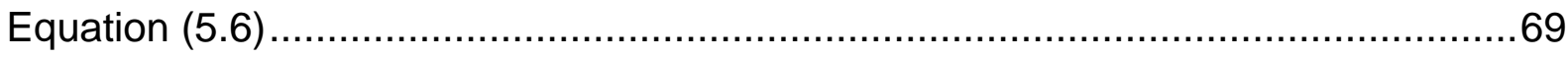

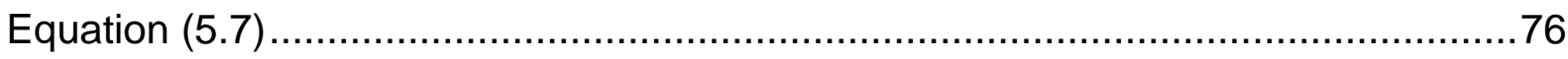

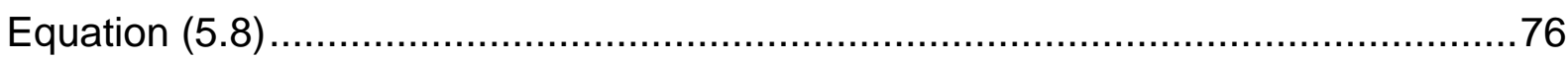

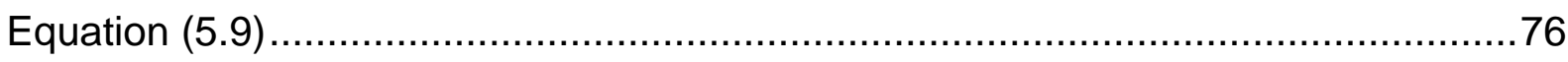

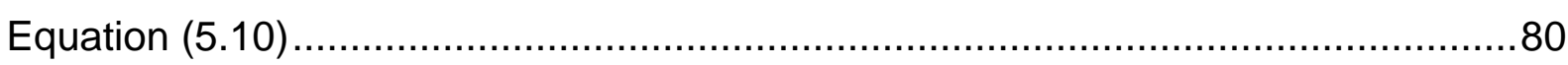

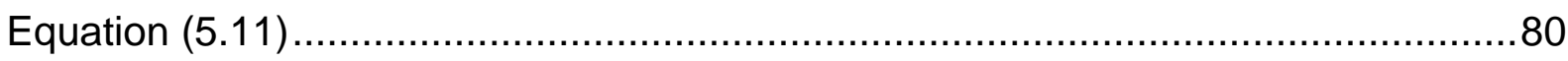

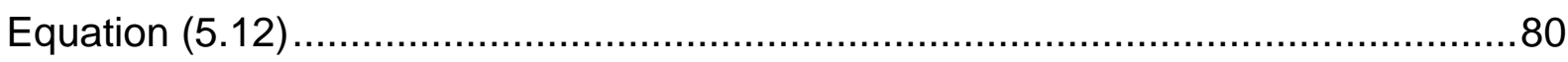

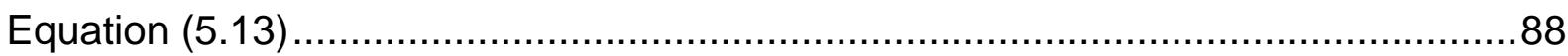

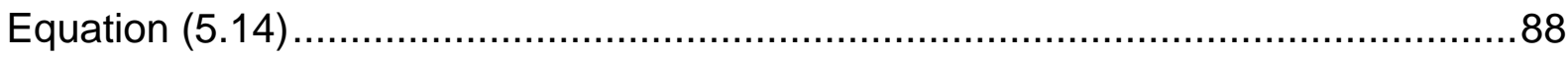

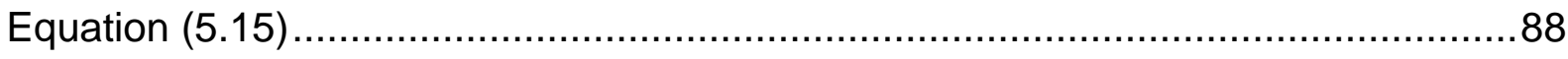

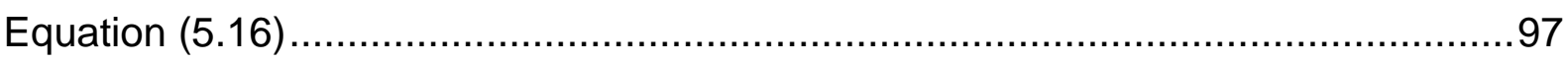




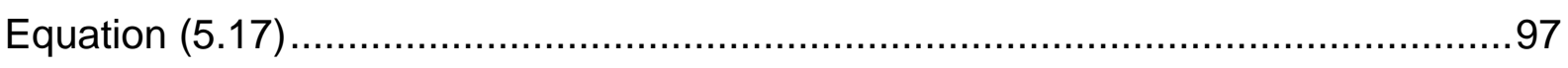

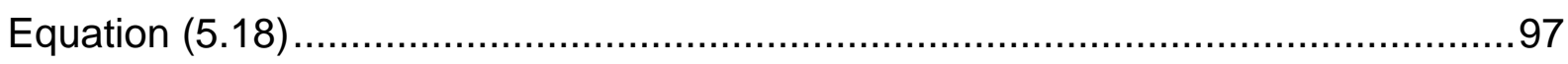

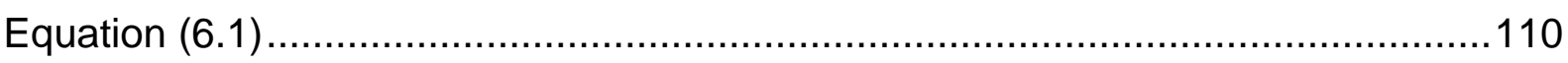




\section{LIST OF ABBREVIATIONS AND ACRONYMS}

$\begin{array}{ll}\text { BAB } & \text { Branch and Bound } \\ \text { BJ } & \text { Box-Jenkins } \\ \text { CV } & \text { Controlled Variable } \\ \text { D-RTO } & \text { Dynamic-RTO } \\ \text { IMC } & \text { Internal Model Control } \\ \text { MILP } & \text { Mixed Integer Linear Programming } \\ \text { MIMO } & \text { Multiple-Input and Multiple-Output } \\ \text { MINLP } & \text { Mixed Integer Nonlinear Programming } \\ \text { MISO } & \text { Multiple-Input and Single-Output } \\ \text { MPC } & \text { Model Predictive Control } \\ \text { MV } & \text { Manipulated Variable } \\ \text { NMPC } & \text { Nonlinear Model Predictive Control } \\ \text { PID } & \text { Proportional-Integral-Derivative } \\ \text { RGA } & \text { Relative Gain Array } \\ \text { RTO } & \text { Real Time Optimization } \\ \text { SISO } & \text { Single-Input and Single-Output } \\ \text { SSE } & \text { Sum of Squared Errors }\end{array}$




\section{LIST OF SYMBOLS}

$\begin{array}{cl}x_{p}(t) & \text { Process state vector } \\ x_{c}(t) & \text { Controller state vector } \\ t & \text { Time vector } \\ r(t) & \text { Reference signal } \\ u(t) & \text { Process input variable vector } \\ j(x) & \text { Objective function } \\ j^{*} & \text { Optimal value of function } j(\cdot) \\ \alpha & \text { Relative (multiplicative) tolerance } \\ \delta & \text { Absolute (additive) tolerance } \\ m_{v}(t) & \text { Manipulate variable vector } \\ G(s) & \text { Process model (continuous time) } \\ H(s) & \text { Disturbance model (continuous time) } \\ G(z) & \text { Process model (discrete time) } \\ H(z) & \text { Disturbance model (discrete time) } \\ d(s) & \text { Disturbance variable vector (continuous time) } \\ d(z) & \text { Disturbance variable vector (discrete time) }\end{array}$




\section{CONTENTS}

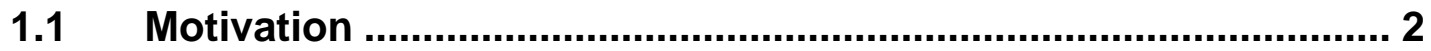

$1.2 \quad$ The Plantwide Control Problem ................................................... 4

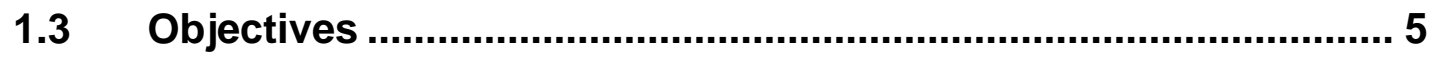

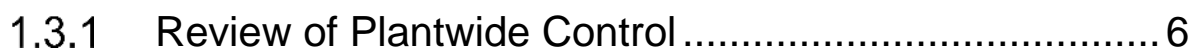

1.3.2 Description and Solution of the Problem of MultiObjective Optimization ...

1.3.3 Formulation of the Requisites of a Plantwide Control Technique 6 5 
2.4 Plantwide Control Benchmarks................................................ 21

2.5 Important Topics in Plantwide Control ..................................... 23

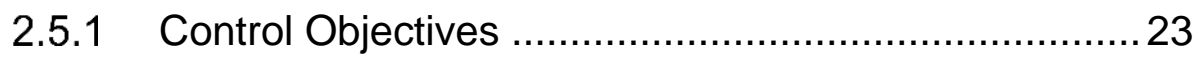

2.5.2 Distributed versus Centralized Approaches ................24

2.5.3 Steady-State and Dynamic Approaches .....................25

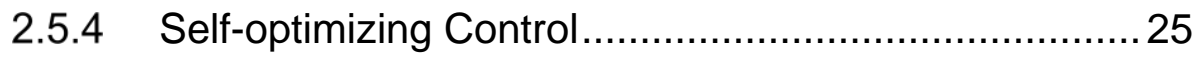

2.5.5 Closed Loops and Degrees of Freedom ....................26

2.5.6 Selection of Controlled Variables ................................27

2.5.7 Control of Recycling Systems ..................................... 28

2.5.8 Control of Unstable Units ............................................29

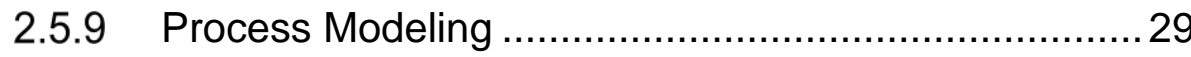

2.5.10 Design of the Plantwide Control System: Before or After the Plant is Built?............................................. 30

2.6 Concluding Remarks.............................................................. 31

3 Theoretical Tools for an Augmented Plantwide Control Design

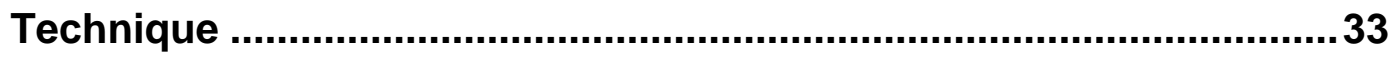

3.1 System Identification for Large Scale Systems .......................... 33

3.2 Optimality in Plantwide Control ................................................. 34

3.2.1 Optimality Sensitivity .................................................. 35

3.2.2 Optimality Robustness .............................................. 35

3.3 Bellman's Principle of Optimality and Plantwide Control .......... 36

3.3.1 Example Scenario with Recycle...................................36

3.3.2 Bellman's Principle of Optimality ............................... 37

3.3.3 Ensuring Optimality in Plantwide Control .....................38

3.4 Nash Equilibrium and Pareto Optimality ..................................... 39

3.5 Cooperation between Multiple Controllers ................................ 39

3.6 Multi-Objective Optimization ................................................... 42

3.6.1 The Multi-Objective Optimization Problem.................. 42

3.6.2 Choice of the Best Solution in a Multi-Objective Optimization Problem................................................ 43

3.6.2.1 Composite Function Methods ...................... 43

3.6.2.2 Goal Programming ....................................... 44 
3.6.2.3 Augmented Lexicographic Multi-Objective Optimization Procedure................................ 45

3.6.2.4 Approaches Comparison.............................. 47

3.6.3 Multi-Objective Optimization in Plantwide Control Design................................................................. 49

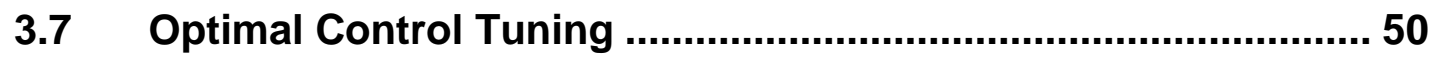

3.7.1 The Optimal Control Tuning Problem...........................50

3.7.2 A Procedure for Control Tuning .................................51

3.7.3 Standard Optimal Tuning Problem Formulation ...........51

3.7.4 Control Tuning in Plantwide Control Design ................53

3.8 Concluding Remarks................................................................ 53

4 Proposed Techniques for Plantwide Control Design.

4.1 Specification of a Novel Plantwide Control Design Technique 55

4.2 Augmented Hierarchical Plantwide Control Design Technique 56

4.3 Optimal Multi-Objective Technique for Integrated Control Structure Selection and Controller Tuning 60

4.4 Concluding Remarks 62

Investigatory Tests and Application Examples .64

5.1 Investigation of Control Structure Selection and Controller Tuning for PID Controllers Applied to Classical Industrial Benchmarks 64

5.1.1 Tests with the Wood and Berry Distillation Column Benchmark. 65

5.1.2 Tests with the III-Conditioned High Purity Distillation Column Benchmark 69

5.1.3 Tests with the Binary Distillation Column Benchmark

5.1.4 Tests with the Industrial Furnace Benchmark ..............75

5.1.5 Tests with the $3 \times 3$ Distillation Column Benchmark .....79

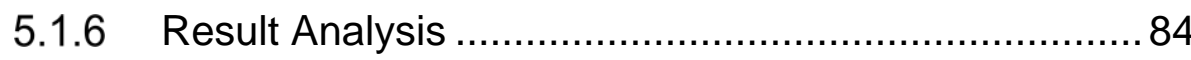

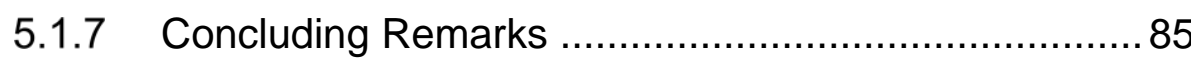


5.2 Applications of the Proposed Techniques

5.2.1 Plantwide Control Design for the HOF Benchmark ..... 86

5.2.1.1 The Shell HOF Benchmark .......................... 87

5.2.1.2 Regulatory Control Layer Design ..................89

5.2.1.3 Supervisory Control Layer Design ............... 92

5.2.1.4 Results for the Shell HOF Benchmark ......... 95

5.2.2 Plantwide Control Design for the FCC Benchmark ..... 95

5.2.2.1 The FCC Benchmark ...................................96

5.2.2.2 Regulatory Control Layer Design .................. 98

5.2.2.3 Supervisory Control Layer Design ............. 100

5.2.2.4 Results for the FCC Benchmark ................ 104

5.2.3 Concluding Remarks .............................................. 105

6 Design of a Plantwide Control System to the Tennessee Eastman Challenge .106

6.1 The Tennessee Eastman Challenge Process............................ 106

6.2 Implementation of the Tennessee Eastman Model.................... 110

6.3 The Augmented Hierarchical Plantwide Control Design Technique

6.4 The Optimal Multi-Objective Technique for Integrated Control Structure Selection and Controller Tuning.................. 112

6.5 The System Identification Platform Used ................................. 112

6.6 Design of the Control System .............................................. 112

6.6.1 I - Specification (Top-Down) Steps .......................... 113

6.6.2 II - Design (Bottom-Up) Steps .................................. 116

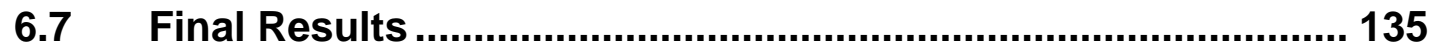

6.7.1 XMEAS 1, XMEAS 2, XMEAS 3 and XMEAS 4 (Gas

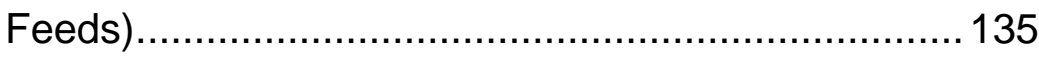

6.7.2 XMEAS 7 (Reactor Pressure) and XMEAS 10 (Purge

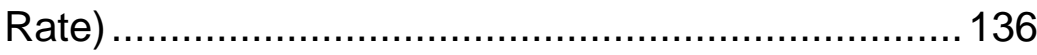

6.7.3 XMEAS 8 (Reactor Level) and XMEAS 11 (Product Separator Temperature) ......................................... 138

6.7.4 XMEAS 9 (Reactor Temperature) ............................ 139 
6.7.5 XMEAS 12 (Product Separator Level) and XMEAS 14 (Product Separator Underflow) ........................... 140

6.7.6 XMEAS 15 (Stripper Level) and XMEAS 17 (Stripper Underflow) ....................................................... 142

6.7.7 XMEAS 18 (Stripper Temperature) and XMEAS 19 (Stripper Steam Flow)

6.7.8 XMEAS 21 (Reactor Cooling Outlet Temperature) ... 144

6.7.9 Concluding Remarks ............................................. 145

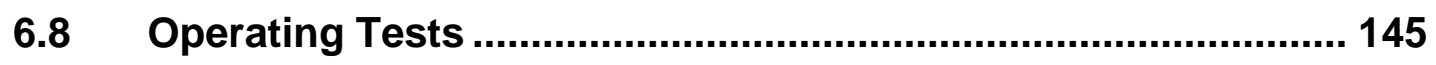

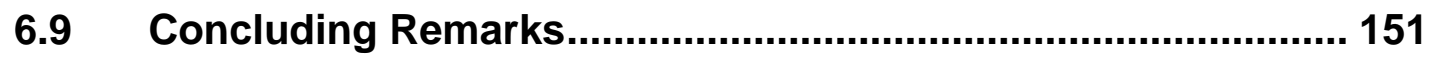

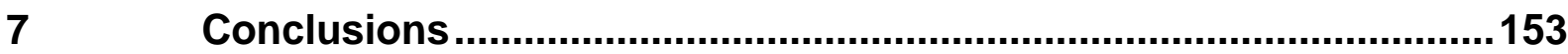

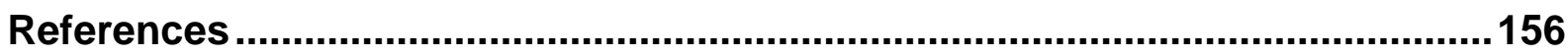

Appendix A - System Identification Procedure ..............................................171

A.1 Identification Experiment............................................................ 171

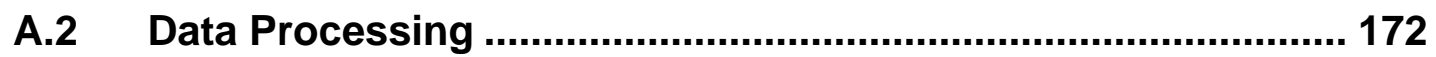

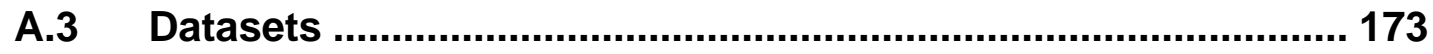

A.4 Model Identification............................................................... 174

A.5 Model Validation and Selection ................................................ 174

A.6 Identified Model .................................................................. 177

Appendix B - Identified Model for the Tennessee Eastman Challenge Process with Regulatory Control in Closed Loop ..........................................178

Index 


\section{Introduction}

A control system regulates the behavior of a dynamical system so that it follows, or gets as close as possible to, a desired specification. The control of a process is performed by the manipulation of some of its variables based on observations of some of the system's outputs.

When the dimension of the system to be controlled increases, so does the complexity of the control problem. The classical approaches become unsolvable for large systems and new ones are needed to design efficient control systems. Such approaches are studied under the subject of Plantwide Control.

Traditionally, control theory faces the control design problem expressed as follows: given a system described by a certain model, a controller that follows a certain specification must be obtained. However, when addressing a whole plant, several problems arise. For example, a complete process model may not be available, the variables to be controlled or manipulated may still need to be chosen, and the control specification can be unclear or incomplete (i.e., specifications are expressed in a process operation or economic manner and not in a precise Control Engineering language). Thus, most traditional control design techniques are unable to deal with problems of large processes.

In several plants, control design is handled as a pure art, rather than a science, based solely on heuristics acquired through experience and trial and error. Although this approach is broadly and successfully applied to design functional control systems that stabilize and improve the operation of the processes, significant gains can be achieved by using a systematic plantwide control design technique that generates a control system that not only automates a plant, but also optimizes it in several ways, from operation to economics.

The problem of designing controllers for complete systems is studied and presented in this thesis, with an emphasis on obtaining a controller that is able not only to stabilize and automatize a process, but also to robustly optimize its behavior according to a given set of feasible specifications.

The main contributions of this work are the presentation of a broad review on Plantwide Control, the identifications of new tools to improve plantwide control design, 
and the proposal of a new Augmented Hierarchical Plantwide Control Design Technique and of a new Optimal Multi-Objective Technique for Integrated Control Structure Selection and Controller Tuning. These proposed techniques include system identification and optimal control tuning as part of the plantwide control design, support multi-objective specifications and are applicable to different plants and controllers. Application examples are also presented to validate and illustrate the proposals.

\subsection{Motivation}

The need for a plantwide control theory was first highlighted in (Foss, 1973): "The central issue to be resolved by the new theories is the determination of the control system structure. Which variables should be measured, which inputs should be manipulated and which links should be made between the two sets? There is more than a suspicion that the work of a genius is needed here, for without it the control configuration problem will likely remain in a primitive, hazily stated and wholly unmanageable form. The gap is present indeed, but contrary to the views of many, it is the theoretician who must close it."

Some decades after this statement, much progress has been achieved in the subject, but the need for new improvements is still present, as stated in (Skogestad, 2000a): "Even though control engineering is well developed in terms of providing optimal control algorithms, it is clear that most of the existing theories provide little help when it comes to making decisions about control structure."

Regarding the current industrial practices, Downs and Skogestad (2011) state that a commonly employed control strategy is to set production rates using the process feed rates and then to design the control system around each unit through the process. Although this approach can be successfully used in many processes, those with less in-process inventory or that are more complex need better approaches.

Another important topic is related to the selection of control objectives. Even though most common control specifications are to regulate variables at a certain level, regulation of all measurable variables is not always necessary. In fact, the choice of variables to be controlled and its control objectives are very complex decisions to be made in control design (Foss, 1973). 
Another important and not broadly considered topic on Plantwide Control is process dynamics. Usually, the design of plantwide control systems focus on steadystate stability and optimality. However, if the control is not perfect, which happens in most situations, this approach fails to provide an optimal system. In fact, process economics is not only a function of steady-state, but also of dynamics for most processes.

A design that presents optimal steady-state operation at nominal point does not guarantee optimal operation in a real scenario with disturbances (Zheng, et al., 1999) and control design techniques that are based solely on steady-state information can result in poor performance in several cases (Skogestad, et al., 1990) and should be avoided.

Regarding the process description needed for a good control design, static information is usually not enough, but a complete model may also be impractical and unnecessary (Foss, 1973). The process model must represent the relevant dynamics and its nature should be determined by the control specifications and the design procedure to be pursued (Foss, 1973), (Juliani, 2012).

Considering the presented aspects, it is noticeable that techniques for plantwide process control design are needed, that result in processes with near-optimal operation and that can be operated without the need of control experts (Downs \& Skogestad, 2011).

Even though plantwide control research started some decades ago, formally with the publication of (Buckley, 1964) and then with the emphasis given in (Foss, 1973), most of the research performed in the topic prior to the year 2000 addressed the problem as the selection of input-output pairing for independent controllers (Stephanopoulos \& $\mathrm{Ng}, 2000$ ). Only recent research has taken optimal operation, multivariable controllers, optimality and other relevant aspects into consideration.

Despite the research and development of new techniques, the literature on Plantwide Control is also relatively scarce when the practical importance of the subject is considered (Gernaey, et al., 2012). Most of the available literature consists of scientific papers, with some book chapters in process engineering books, such as (Seider, et al., 2004), and only a few dedicated books, namely, (Buckley, 1964), 
(Luyben, et al., 1998), (Erickson \& Hedrick, 1999) and (Rangaiah \& Kariwala, 2012). The latter presents a summary of the number of publications on the subject in the last two decades, as reproduced in Figure 1.1.

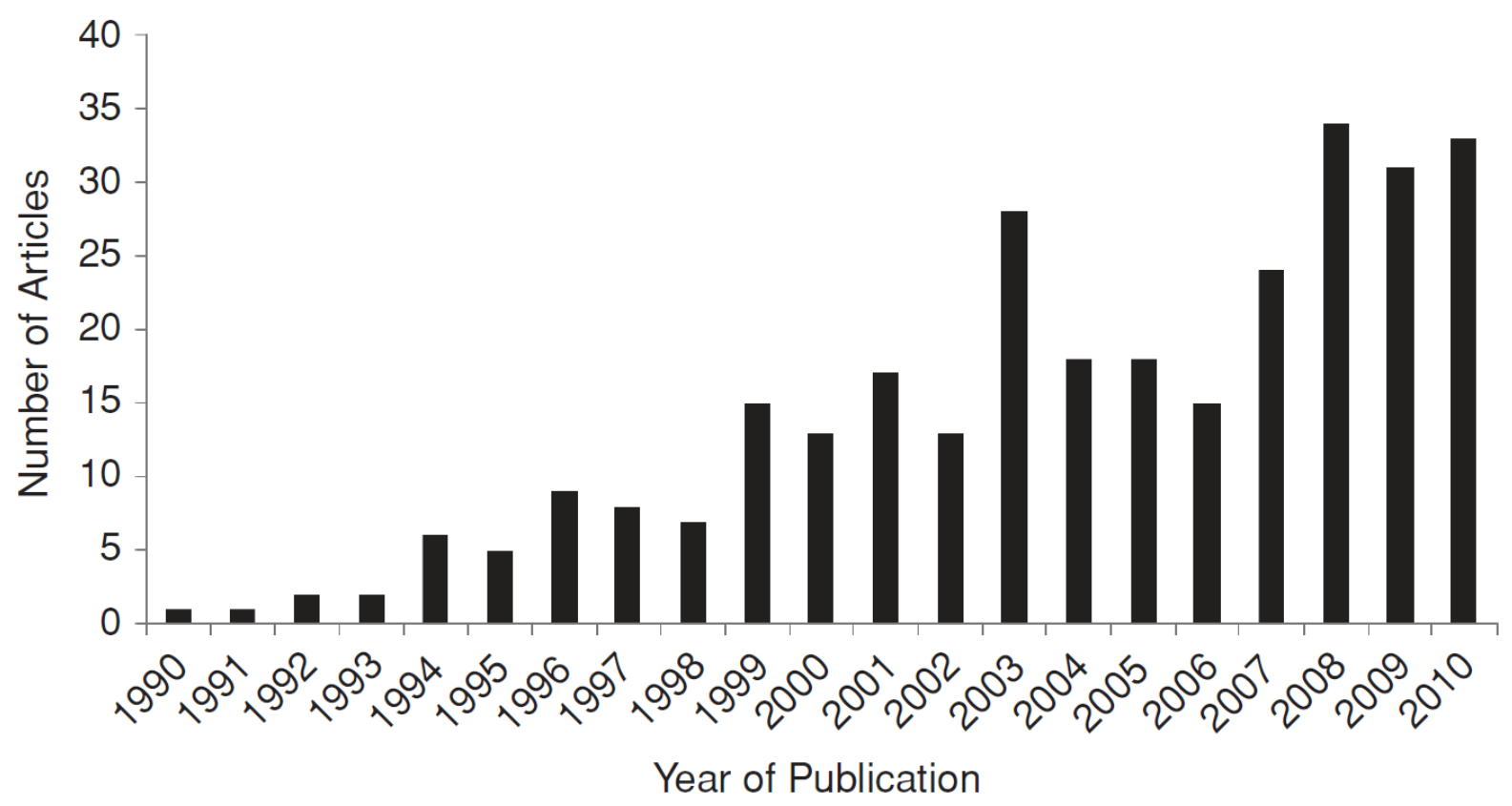

Figure 1.1 - Number of Plantwide Control articles published during the period 1990-2010 (Rangaiah \& Kariwala, 2012).

This demonstrates that not only new studies in the subject are needed, but also that a comprehensive literature could greatly enhance the development and applications of plantwide control.

The present work is focused on process control, area in which most applications and studies of plantwide control design are performed. However, these design approaches also have application in other areas (van de Wal \& Jager, 1995), such as aircraft control (Reeves, 1991), (Samar \& Postlethwaite, 1994), design of active suspensions for vehicles (Al-Sulaiman \& Zaman, 1994), (van de Wal, 1994), control of flexible structures (Abdel-Mooty \& Roorda, 1991), (Byeongsik, et al., 1994), (Norris \& Skelton, 1989) and satellite attitude control (Müller \& Weber, 1972).

\subsection{The Plantwide Control Problem}

A brief definition of the Plantwide Control problem is presented. Although the problem itself is not perfectly defined, the following summary presents a concise idea 
of it: In a mathematical sense, the plantwide control problem is a formidable and almost hopeless combinatorial problem involving a large number of discrete decision variables. In addition, the problem has been poorly defined in terms of its objectives (Skogestad, 2012), (Gernaey, et al., 2012). Generally, it involves the following tasks (Foss, 1973), (Skogestad \& Postlethwaite, 1996):

1. Selection of control objectives;

2. Selection of controlled variables (CVs);

3. Selection of manipulated variables (MVs);

4. Selection of extra measurements;

5. Selection of control configuration (i.e., the structure of the overall controller that interconnects the controlled, manipulated and measured variables);

6. Selection of controller type.

Briefly, Plantwide Control design includes all structural decisions of a control system, but not the actual design of the controllers.

\subsection{Objectives}

This thesis has as main goal the proposal of a new plantwide control design technique. An approach is sought that is well defined and capable of been applied without much knowledge of the process, but that allows the use of process knowledge to improve results. The technique must provide a control system that not only stabilizes and automates a process, but also drives it to optimal and robust operation. Finally, robustness and optimality should be possible to be defined as a single performance function (usually an economic index) or as a set of multiple performance indexes.

To achieve this goal, the following set of objectives is proposed:

O-1. Review of Plantwide Control;

O-2. Description and solution of the problem of multi-objective optimization;

O-3. Formulation of the requisites of a plantwide control technique;

O-4. Proposal of a novel plantwide control technique;

O-5. Application of the proposed approach to an industrial benchmark. 
To achieve these objectives, some tasks are enumerated for each of them. In the following sections, these five objectives are detailed and 20 tasks associates with them are presented.

\subsubsection{Review of Plantwide Control}

As mentioned in the Introduction, the literature in Plantwide Control is scarce, especially when compared with other topics in Control Engineering. A broad review on the topic is then needed for this work. For this review, the following tasks are contemplated:

O1-T1. Enumeration of previous reviews on Plantwide Control;

O1-T2. Enumeration of existing plantwide control techniques;

O1-T3. Enumeration of large scale benchmarks;

O1-T4. Identification of open issues in Plantwide Control; and

O1-T5. Description of the most relevant plantwide control techniques.

\subsubsection{Description and Solution of the Problem of Multi-Objective Optimization}

When designing a control system, multiple process characteristics can present themselves as good candidates to be optimized. However, the classical optimization theory only allows the optimization of a single performance index and it is necessary to choose a single index, or to build an index composed of partial indexes to be optimized. This problem is analyzed and a true multi-objective optimization approach is proposed. The related tasks are:

O2-T1. Description and analysis of the multi-objective optimization problem;

O2-T2. Proposal of a technique for true multi-objective optimization;

O2-T3. Discussion of the proposed technique; and

O2-T4. Description of the application of the proposed multi-objective optimization approach to control design. 


\subsubsection{Formulation of the Requisites of a Plantwide Control Technique}

Along the development of the subject, each new design technique seeks an improvement in some aspect. In order to propose a technique that is an innovative one, some characteristics are sought, and, to achieve that, the following tasks are initially proposed:

O3-T1. Identification of possible contributions;

O3-T2. Description of optimality and robustness;

O3-T3. Description of Bellman's principle of optimality;

O3-T4. Discussion about cooperation between independent controllers; and O3-T5. Presentation of an optimal control tuning technique.

\subsubsection{Proposal of a Novel Plantwide Control Technique}

Considering the performed analysis and employing the presented theoretical tools, a novel plantwide control technique is presented. For this, the following tasks are selected:

O4-T1. Proposal of a novel plantwide control technique;

O4-T2. Analysis and discussion of the proposed technique.

\subsubsection{Application of the Proposed Approach to an Industrial Benchmark}

In order to assess the applicability, advantages and limitations of the proposed technique, it is applied to a selected industrial benchmark. The tasks related to this objective are:

O5-T1. Selection of a benchmark;

O5-T2. Application of the proposed technique to the selected benchmark;

O5-T3. Tests of the resulting control system; and

O5-T4. Analysis of the results and evaluation of the technique. 


\subsection{Organization of the Thesis}

This thesis is organized as follows.

In Chapter 2, Review on Plantwide Control, the major reviews on the subject previously published are summarized and updated. Several works are considered in this review, which seeks to present a concise introduction to the topic for researchers and plant engineers and also to summarize recent advances for those familiar with Plantwide Control. The most important topics on the subject and the most relevant plantwide control design techniques currently available are also presented.

In Chapter 3, Theoretical Tools for an Augmented Plantwide Control Design Technique, some practices and theories are presented and their application to improve plantwide control design is discussed. System Identification, optimality, Bellman's principle of optimality, Nash equilibrium, Pareto optimality, cooperation between multiple controllers and optimal control tuning are addressed and a multi-objective optimization procedure is proposed.

In Chapter 4, Proposed Techniques for Plantwide Control Design, a novel plantwide design technique is specified and proposed. An optimal multi-objective technique for integrated control structure selection and controller tuning is also proposed and described.

In Chapter 5, Investigatory Tests and Application Examples, the proposed techniques are applied to some benchmarks to illustrate and validate its applicability.

In Chapter 6, Design of a Plantwide Control System to the Tennessee Eastman Challenge, a complete plantwide control design is performed to a classic industrial benchmark.

Finally, in Chapter 7, Conclusions, the work is summarized and the obtained results and contributions are analyzed. 


\section{Review on Plantwide Control}

This chapter presents a broad review of Plantwide Control, presenting both an introductory view for those new to the subject and also summarizing recent advances for those familiar with it. Previous reviews were studied, summarized and updated with new publications in the area. The major advances since the beginning of its formal studies are described, with the highlighting of the most noticeable contributions. Plantwide control architectures, design techniques and some important open research topics are addressed. Additionally, notorious literature and benchmarks are enumerated.

Classically, plantwide control design is the selection of controlled variables (CVs), selection of manipulated variables (MVs), selection of extra measurements, selection of control configuration and selection of controller type (Skogestad, 2012). In other words, it typically includes all structural decisions of the control systems, but not the actual design of the system.

Two important introductory works to Plantwide Control are (Foss, 1973) and (Stephanopoulos, 1983), in which the authors perform critical analysis of classical process and control engineering and highlight some of its weaknesses and the need for plantwide procedures. Although these works were published four and three decades ago, respectively, their statements continue to be true and a motivation for research topics that are still open.

The major reviews published on the subject are: (Findeisen, et al., 1980), (Morari, 1982), (Stephanopoulos, 1983), (Balchen \& Mummé, 1988), (Rijnsdorp, 1991), (Rinard \& Downs, 1992), (van de Wal \& Jager, 1995), (Skogestad \& Postlethwaite, 1996), (Luyben, et al., 1998), (Larsson \& Skogestad, 2000) and (Vasudevan, et al., 2009).

Consolidated practices and the most noticeable research results on Plantwide Control are presented in a few dedicated books, namely: (Buckley, 1964), (Luyben, et al., 1998), (Erickson \& Hedrick, 1999) and (Rangaiah \& Kariwala, 2012).

In this chapter, the main topics of the subject are summarized and updated. ${ }^{1}$

${ }^{1}$ The present review was published in (Juliani \& Garcia, 2017b). 


\subsection{Plantwide Control Architectures}

A plantwide control system, regardless of the design technique employed to build it, can be classified according to its level of integration into some main architectures, as depicted in Figure 2.1 (Ochoa, et al., 2010).

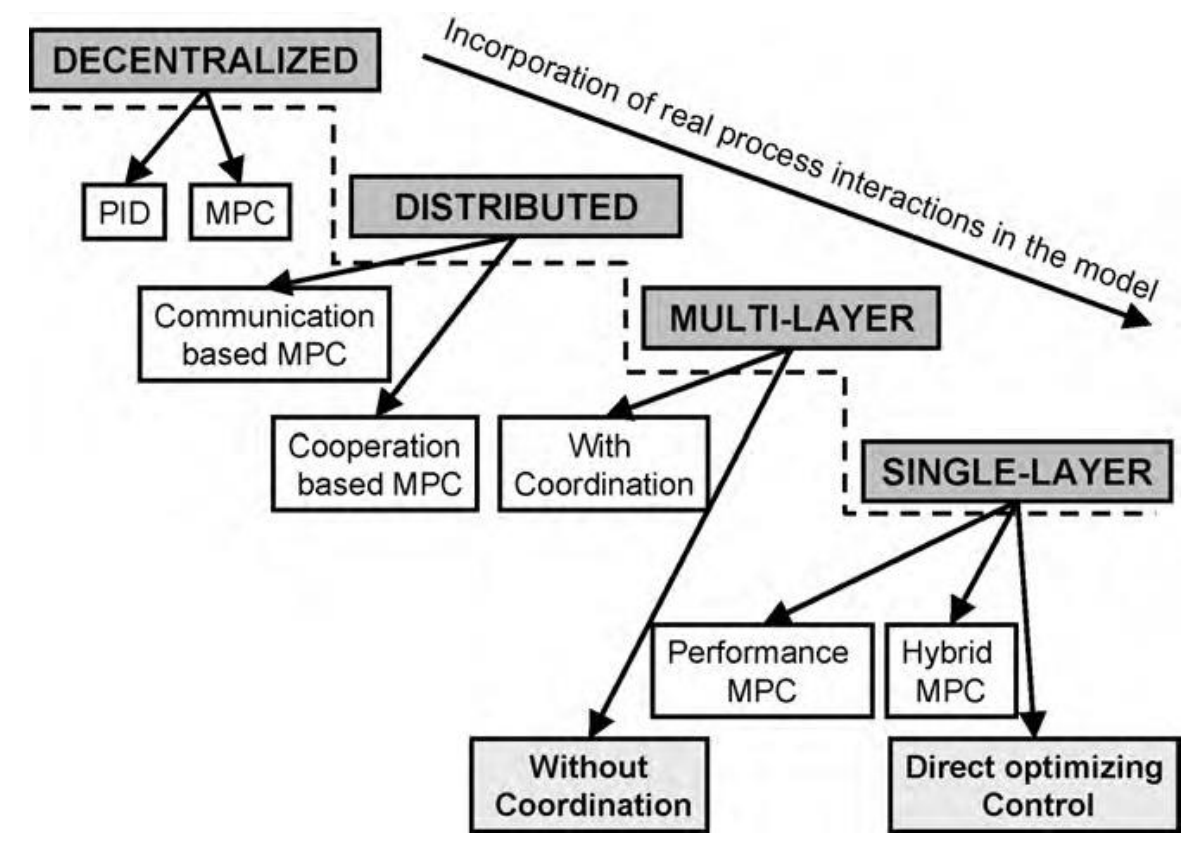

Figure 2.1 - Classification of plantwide control architectures (Ochoa, et al., 2010).

The decentralized architecture consists of independent controllers, such as PID (Proportional-Integral-Derivative) or MPC (Model Predictive Control) that does not share any kind of information, i.e., does not communicate with each other when operating, even if the selection of their controlled and manipulated variables or their tuning considers the process interactions.

Distributed architectures consist of multiple controllers that interact for a better global performance. The two main architectures in this category are the communication-based MPC and the cooperation-based MPC which respectively employ controllers with a local objective function and controllers with a copy of the total objective function for the complete plant (Rawlings \& Steward, 2008), (Ochoa, et al., 2010).

The multilayer architecture contains algorithms connected in a hierarchical manner, such that higher-level controllers coordinate the lower-level ones, which deal with more detailed dynamics (Ochoa, et al., 2010). This architecture may or may not 
include a coordination layer. In the multilayer architecture with a coordination layer, this layer is found between the higher layer, usually an RTO (Real-Time Optimization) and the lower layer, usually an MPC, and is responsible for managing the information coming from both layers and finding locally feasible references for the MPC that are close to the global solution found by the RTO (Ochoa, et al., 2010). When no coordination is used, the RTO is replaced by a D-RTO (Dynamic-RTO), which sends the references directly to the controllers, such as MPC or NMPC (Nonlinear Model Predictive Control). Figure 2.2 depicts a typically applied multilayer architecture with common layers and time scales (Skogestad, 2000a).

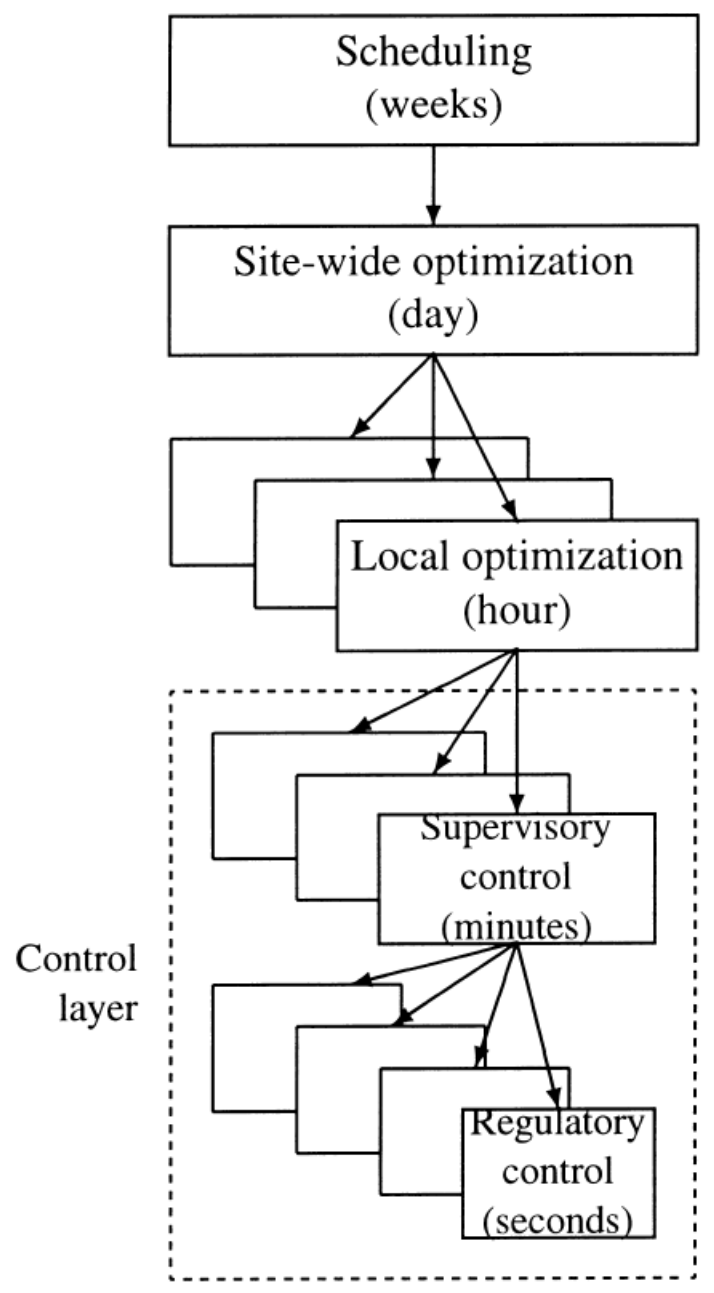

Figure 2.2 - Typical hierarchical control (Skogestad, 2000a).

Finally, the single-layer architecture applies a single large controller to regulate the whole process. A first relevant group in this category is the performance MPC, which uses an MPC with a performance-type objective function, in which the tracking of reference values for the controlled and manipulated variables is penalized (Ochoa, et al., 2010). A second group of controllers, hybrid MPC (Engell, 2007), include an 
economic penalization term in the objective function along with the performance term. The last group, direct optimizing control, uses a pure economic objective function, with the control specifications used as constraints.

\subsection{Plantwide Control Design Techniques}

Since the start of formal studies on Plantwide Control, many design techniques have been proposed. In this section, these techniques are enumerated, and the most noteworthy are detailed.

Plantwide control design techniques are usually organized in three categories. The first one is process-oriented, without much systematic procedure. The second category is mathematically oriented, usually referred to as control structure design. Finally, the third one contains approaches that are hybrids of the two previous categories. All of these approaches present some advantages and limitations.

The process-oriented approaches are usually easy to understand and to implement for process engineers, but are greatly dependent on experience and process knowledge and often lead to non-optimal solutions. The main process-oriented procedures are discussed in (Buckley, 1964), (Shinskey, 1984), (Douglas, 1988), (Downs, 1992), (Luyben, et al., 1997), (Luyben, et al., 1998), (Seider, et al., 2004) and (Konda, et al., 2005).

Mathematical and optimization-based methodologies may not be easy to formulate and require extensive computation to be solved, but result in more reliable and rigorous solutions (Vasudevan, et al., 2009). Mathematically oriented procedures are proposed in (Narraway \& Perkins, 1993), (Hansen, et al., 1998), (Heath, et al., 2000), (Groenendijk, et al., 2000), (Dimian, et al., 2001), (Kookos \& Perkins, 2001), (Kookos \& Perkins, 2002), (Chen \& McAvoy, 2003), (Chen, et al., 2003), (Cao \& Saha, 2005), (Engell, 2007), (Cao \& Kariwala, 2008), (Molina, et al., 2011), (Sharifzadeh \& Thornhill, 2012) and (Psaltis, et al., 2013).

Hybrid approaches try to merge the best of the former two, employing some systematic mathematical approach that includes the use of process knowledge. Procedures in this category include those described by (Zheng, et al., 1999), 
(Jørgensen \& Jørgensen, 2000), (Zhu, et al., 2000), (Larsson \& Skogestad, 2000), (Skogestad, 2000a), (Skogestad, 2000b) (Robinson, et al., 2001), (Wang \& McAvoy, 2001), (Vasbinder \& Hoo, 2003), (Skogestad, 2004), (Ward, et al., 2006) and (Baldea, et al., 2008).

The major recent plantwide control methodologies are briefly described in Table 2.1 (Vasudevan, et al., 2009).

Table 2.1 - Plantwide control design techniques proposed between 2000 and 2009 (Vasudevan, et al., 2009).

\begin{tabular}{|c|c|}
\hline References & Main Features \\
\hline $\begin{array}{l}\text { (Skogestad, 2000a), } \\
\text { (Skogestad, 2000b), } \\
\text { (Skogestad, 2004) }\end{array}$ & $\begin{array}{l}\text { In this self-optimizing control methodology, control system } \\
\text { design is divided into three layers based on time scale: local } \\
\text { optimization, supervisory control, and regulatory control. }\end{array}$ \\
\hline $\begin{array}{l}\text { (Jørgensen \& } \\
\text { Jørgensen, 2000) }\end{array}$ & $\begin{array}{l}\text { The control structure selection problem is formulated as an } \\
\text { MILP (Mixed-Integer Linear Programming) problem } \\
\text { employing cost coefficients. }\end{array}$ \\
\hline (Zhu, et al., 2000) & Hybrid strategy integrating linear and nonlinear MPC. \\
\hline $\begin{array}{l}\text { (Groenendijk, et al., } \\
\text { 2000), (Dimian, et al., } \\
\text { 2001) }\end{array}$ & $\begin{array}{l}\text { Combination of steady-state and dynamic controllability } \\
\text { analysis for evaluating the dynamic inventory of impurities. }\end{array}$ \\
\hline $\begin{array}{l}\text { (Robinson, et al., } \\
\text { 2001) }\end{array}$ & $\begin{array}{l}\text { Design of a decentralized plantwide control system using an } \\
\text { optimal control-based approach. }\end{array}$ \\
\hline $\begin{array}{l}\text { (Kookos \& Perkins, } \\
\text { 2001) }\end{array}$ & $\begin{array}{l}\text { Mixed-integer nonlinear programming problem to minimize } \\
\text { overall interaction and sensitivity of the closed-loop system } \\
\text { to disturbances. }\end{array}$ \\
\hline $\begin{array}{l}\text { (Wang \& McAvoy, } \\
\text { 2001) }\end{array}$ & $\begin{array}{l}\text { MILP problem in each of the three stages of the control } \\
\text { system synthesis: control of safety, production, and } \\
\text { remaining process variables. }\end{array}$ \\
\hline $\begin{array}{l}\text { (Chen \& McAvoy, } \\
\text { 2003), (Chen, et al., } \\
\text { 2003) }\end{array}$ & $\begin{array}{l}\text { This hierarchical method was based on linear dynamic } \\
\text { process models and optimal static output feedback } \\
\text { controllers and later extended to processes with multiple } \\
\text { steady states. }\end{array}$ \\
\hline
\end{tabular}




\begin{tabular}{l|l}
\hline \multicolumn{1}{c|}{ References } & \multicolumn{1}{c}{ Main Features } \\
\hline (Vasbinder \& Hoo, & $\begin{array}{l}\text { Decision-based approach in which the plant is decomposed } \\
\text { into smaller modules using a modified analytical hierarchical } \\
\text { process. }\end{array}$ \\
\hline (Cao \& Saha, 2005), & This is an improved and more efficient algorithm of the \\
(Cao \& Kariwala, & "branch and bound (BAB)" method for control structure \\
2008) & $\begin{array}{l}\text { screening. Later, the authors presented a bidirectional BAB } \\
\text { algorithm for efficient handling of large-scale processes. }\end{array}$ \\
\hline (Konda, et al., 2005) & $\begin{array}{l}\text { Integrated framework of simulation and heuristics that uses } \\
\text { steady-state and dynamic simulation to take or support the }\end{array}$ \\
& \begin{tabular}{l} 
Cocisions taken by heuristics. \\
\hline (Baldea, et al., 2008)
\end{tabular} \\
\hline
\end{tabular}

\subsection{Main Plantwide Control Design Techniques}

With the continuous increase in the size and complexity of industrial plants, and the increasing need for operational safety and efficiency, the use of plantwide control is acquiring more and more importance. Next, the design techniques most capable of dealing with the challenges of modern processes are briefly described.

\subsubsection{Optimization Procedure by Narraway and Perkins (1993)}

A control structure selection technique based on optimization is proposed in (Narraway \& Perkins, 1993) and demonstrated in (Narraway \& Perkins, 1994). In this approach, the problem is written as a classical optimization formalization, in which dynamic optimization and mixed integer nonlinear programming (MINLP) are employed to determine the best control structure, according to an economic objective function.

Although very direct and precise, this technique cannot be efficiently applied to large systems due to the high complexity of the resulting optimization problem, being an approach suitable to design control systems for simple plant units. 


\subsubsection{Luyben's Nine-Step Plantwide Control Procedure (1997)}

The first systematic plantwide control procedure was suggested in (Luyben, et al., 1997), (Luyben, et al., 1998) and consists of the following nine steps:

1. Establish the control objectives;

2. Determine the control degrees of freedom;

3. Establish the energy management system;

4. Set the production rate;

5. Control the product quality and handle safety, environmental and operation constraints;

6. Fix a flow in every recycle loop and control inventories;

7. Check component balances;

8. Control individual unit operations;

9. Optimize the economics and improve dynamic controllability.

This procedure is noticeable for being a first systematic approach to solve the problem, but has a major weakness of only including economics in the last step, which can result in a control structure with poor performance (Gernaey, et al., 2012).

\subsubsection{Hierarchical Procedure by Zheng, Mahajanam and Douglas (1999)}

A hierarchical procedure for plantwide control synthesis was proposed in (Zheng, et al., 1999), in which the problem is solved not by evaluating all the possible alternatives (exhaustively or by optimization), but rather by its decomposition into a hierarchy of decisions based on process economics, in which the decisions with greater economic impact are considered first and then only the most economically attractive alternatives are kept for the next decisions.

The steps of the referenced hierarchical procedure are the following (Zheng, et al., 1999):

1. Steady-state robust feasibility;

2. Selection of controlled variables;

3. Steady-state control structure screening;

4. Dynamic control structure synthesis; 


\section{Economic ranking; and \\ 6. Dynamic simulations.}

Of particular interest is the first step, which deals with the robust feasibility of the process by solving the following problem: let $\mathcal{U}, \mathcal{Y}$ and $\mathcal{D}$ be the sets of allowed inputs, allowed outputs and expected disturbances, respectively, and consider the steady-state model of the process. Then the system is feasible if there is some $u \in$ $\mathcal{U} \in \mathbb{R}^{n_{u}}$ that results in $y \in \mathcal{Y} \in \mathbb{R}^{n_{y}}$ for any $d \in \mathcal{D} \in \mathbb{R}^{n_{d}}$.

The great advantage of a hierarchical approach compared to a pure optimization one is that while a direct optimization is usually too complex to be solved due to the necessity of an accurate process model and the large number of decision variables involved (Zheng, et al., 1999), the hierarchical approach allows a rigorous optimization procedure to be applied in several stages, without creating a mathematically unsolvable problem.

\subsubsection{Optimization Procedure by Jørgensen and Jørgensen (2000)}

A concise mathematical approach to the plantwide control problem is presented in (Jørgensen \& Jørgensen, 2000). In this method, the decentralized control configuration is obtained by the solution of the MILP problem (2.1).

subject to:

$$
\min _{p} \phi(p)=\sum_{\mathrm{i}} \sum_{j} c_{i j} p_{i j}
$$

$$
\begin{array}{lc}
\sum_{j} p_{i j}=1 & \forall i \\
\sum_{i} p_{i j} \leq 1 & \forall j \\
p_{i j} \in\{0,1\} & \forall i, j
\end{array}
$$

in which $p_{i j}$ indicates the pairing between output $i$ and input $j, \phi(p)$ represents the sum of relative interaction of a given control configuration. The constraints ensure that each output is assigned to a single input, and that each input is assigned up to a single output. For the objective function $c_{i j}$, forms (2.2),(2.3) and (2.4) are available, depending on the chosen performance criteria (Jørgensen \& Jørgensen, 2000). 


$$
\begin{gathered}
c_{i j}=\left\{\begin{array}{ll}
\left|\phi_{i j}(0)\right| & \phi_{i j}(0)>-1+\left|\frac{\Delta g_{i j}(0)}{g_{i j}(0)}\right| \\
\infty & \text { otherwise }
\end{array} \mid\right. \\
c_{i j}=\theta_{\mathrm{ij}}+2 \sum_{k i j} \frac{\operatorname{Re}\left(\xi_{k_{i j}}\right)}{\left|\varsigma_{k_{i j}}\right|^{2}} \\
c_{i j}=\left(\frac{1}{2 \pi} \int_{-\infty}^{\infty}\left|\frac{1-\hat{g}_{A i j}(i \omega)}{+\hat{g}_{A i j}(i \omega) \phi(i \omega) i \omega} \cdot \frac{1}{i \omega}\right| d \omega\right)^{\frac{1}{2}}
\end{gathered}
$$

Although this technique is limited to linear systems and lacks generalization, it is a very straightforward and rigorous approach.

\subsubsection{Skogestad's Seven-Step Plantwide Control Procedure (2000)}

The seven-step plantwide control design procedure of Skogestad was inspired in Luyben's procedure and is divided into a top-down part, mainly concerned with steady-state economics, and a bottom-up part, mainly concerned with stabilization and pairing of loops (Larsson \& Skogestad, 2000), (Skogestad, 2000a), (Skogestad, 2000b), (Skogestad, 2004a).

The top-down steps are (Skogestad, 2012):

1. Define operational objectives (economic cost function $J$ and constraints);

2. Identify steady-state degrees of freedom $u$ and determine the optimal steadystate operation conditions, including active constraints;

3. Identify candidate measurements $y$ and select primary controlled variables $C V_{1}=H y$ (decision 1);

4. Select the location of TPM (throughput manipulator) (decision 3).

The bottom-up steps are (Skogestad, 2012):

5. Select the structure of regulatory (stabilizing) control layer:

a) Select "stabilizing" controlled variables $\mathrm{CV}_{2}=\mathrm{H}_{2} y$ (decision 2);

b) Select inputs and "pairings" for controlling $\mathrm{CV}_{2}$ (decision 4).

6. Select the structure of supervisory control layer;

7. Select structure of (or assess need for) optimization layer (RTO). 
This approach was presented in a series of papers, which include some applications to large-scale processes. A verbose analysis of each step with practical consideration were presented in (Skogestad, 2002) and in (Gernaey, et al., 2012).

The main advantage of this method is that it is a systematic approach that does not heavily rely on either a heuristic knowledge of the process or a vast process control engineering experience. It also includes an economic optimization since the start of the procedure.

\subsubsection{Integrated Framework of Simulation and Heuristics by Konda, Rangaiah and Krishnaswamy (2005)}

A hybrid plantwide control technique is presented in (Konda, et al., 2005) as an improvement of Luyben's 9-step heuristic procedure (Luyben, et al., 1998). This is an interesting approach because of the use of heuristics with the aid of computer simulation in each of its steps.

The steps of this integrated framework are (Konda, et al., 2005):

1. Definition of plantwide control objectives;

2. Determination of control degrees of freedom;

3. Identification and analysis of plantwide disturbances;

4. Setting of performance and tuning criteria;

5. Selection of production rate manipulator;

6. Selection of product quality manipulator;

7. Selection of manipulators for more severe controlled variables;

8. Selection of manipulators for less severe controlled variables;

9. Design of control for unit operations;

10. Checking of component material balances;

11. Analysis and treatment of effects due to integration and recycles; and

12. Enhancement of control system performance, if possible.

While this approach is similar to Luyben's and Skogestad's procedures, the use of simulation in each step to aid and validate design decisions is a very interesting and useful contribution. 


\subsubsection{Optimal Selection of Control Structure Using a Steady-State Inversely Controlled Process Model by Sharifzadeh and Thornhill (2011)}

A simplified direct optimization framework is presented in (Sharifzadeh \& Thornhill, 2012). This framework applies the concepts of perfect control and that the economics are solely determined by steady-state to simplify the problem.

The formulation of this optimization framework is presented in the form (2.5) (Sharifzadeh \& Thornhill, 2011).

subject to:

$$
\min E\left\{J\left(\chi_{c}, \mu\right\}\right.
$$

$$
\begin{gathered}
f\left[x, z, u, y, \chi_{p} . p\right]=0 \\
h\left[x, z, u, y, \chi_{p}, p\right]=0 \\
g\left[x, z, u, y, \chi_{p}, p\right] \leq 0 \\
\psi[\mu]=0 \\
\chi_{c, k} \times\left(y_{i}-\eta_{i}\right)=0 \\
\Omega\left(\chi_{c, k}\right) \geq 0 \quad k \in K
\end{gathered}
$$

in which $\chi_{c, k}$ are binary variables for the selection of controlled variables, $x$ are the process states, $z$ are the process algebraic variables, $u$ is the vector of candidate manipulated variables, $y$ is the vector of candidate controlled variables, $\mu$ is the vector of stochastic disturbance variables, $\chi_{p}$ is the vector of structural process variables, $f[\ldots]=0$ is the vector of the process differential equations, $h[\ldots]=0$ is the vector of process algebraic equations, $g[\ldots] \leq 0$ are the inequality constraints and $\Omega(\cdot)$ represents the vector of inequality constraints. The minimized objective function is the expected value $E\left\{J\left(\chi_{c}, \mu\right\}\right.$.

This is remarkably a well-defined optimization formulation for the plantwide design problem, with the advantage of including all decisions in a single problem. However, this framework is restricted to linear (or linearized) models and it is limited to processes in which the dynamics and disturbances are negligible to the operation cost, with the economics determined solely by the steady-state. Furthermore, it employs the concept of perfect control, i.e., that the controllers keep the controlled variables at their setpoint all the time, which is a very poor and restrictive concept, as real control is never perfect and approaching a perfect behavior can be very costly. 


\subsubsection{Qualifications of the Described Techniques}

The qualifications of the described techniques are summarized in Table 2.2.

Table 2.2 - Qualifications of the described plantwide control design techniques.

\begin{tabular}{|c|c|}
\hline Tech & Qualifications \\
\hline $\begin{array}{l}\text { Optimization Procedure } \\
\text { by Narraway and Perkins } \\
\text { (1993) }\end{array}$ & $\begin{array}{l}\text { This technique is direct and precise but cannot be } \\
\text { efficiently applied to large systems. It provides a formal } \\
\text { MINLP optimization problem that is a good approach to } \\
\text { design control systems for simple plant units. }\end{array}$ \\
\hline $\begin{array}{l}\text { Luyben's Nine-Step } \\
\text { Plantwide Control } \\
\text { Procedure (1997) }\end{array}$ & $\begin{array}{l}\text { It is a systematic procedure to design a plantwide } \\
\text { control system. However, it only includes economic } \\
\text { optimization in its last step, which can limit the final } \\
\text { economic performance. }\end{array}$ \\
\hline $\begin{array}{l}\text { Hierarchical Procedure } \\
\text { by Zheng, Mahajanam } \\
\text { and Douglas (1999) }\end{array}$ & $\begin{array}{l}\text { This technique creates a hierarchical optimization to } \\
\text { determine the control structure. It allows a rigorous } \\
\text { optimization procedure without creating a } \\
\text { mathematically unsolvable problem. }\end{array}$ \\
\hline $\begin{array}{l}\text { Optimization Procedure } \\
\text { by Jørgensen and } \\
\text { Jørgensen (2000) }\end{array}$ & $\begin{array}{l}\text { This method finds a control structure by the solution of } \\
\text { a MILP optimization problem. This is a rigorous and } \\
\text { direct approach, but it is limited to linear systems. }\end{array}$ \\
\hline $\begin{array}{l}\text { Skogestad's Seven-Step } \\
\text { Plantwide Control } \\
\text { Procedure (2000) }\end{array}$ & $\begin{array}{l}\text { This method proposes a systematic approach to design } \\
\text { plantwide control systems, including an economic } \\
\text { optimization of the process. }\end{array}$ \\
\hline $\begin{array}{l}\text { Integrated Framework of } \\
\text { Simulation and Heuristics } \\
\text { by Konda, Rangaiah and } \\
\text { Krishnaswamy (2005) }\end{array}$ & $\begin{array}{l}\text { This is a systematic approach similar to Luyben's and } \\
\text { Skogestad's procedures. It employs simulations of the } \\
\text { process to validate the design decisions. }\end{array}$ \\
\hline $\begin{array}{l}\text { Optimal Selection of } \\
\text { Control Structure Using a } \\
\text { Steady-State Inversely } \\
\text { Controlled Process Model } \\
\text { by Sharifzadeh and } \\
\text { Thornhill (2011) }\end{array}$ & $\begin{array}{l}\text { This method proposes a well-defined optimization } \\
\text { formulation for the plantwide control design. It is } \\
\text { restricted to linear models and it is limited to processes } \\
\text { in which the economics is determined solely by the } \\
\text { steady-state. Its main disadvantage is the reliance on } \\
\text { the concept of perfect control. }\end{array}$ \\
\hline
\end{tabular}




\subsection{Plantwide Control Benchmarks}

Benchmarks are very useful to test and compare control techniques. Plantwide benchmarks are characterized by their large number of variables and/or high complexity. The major benchmark processes employed in Plantwide Control studies are summarized in Table 2.3, originally presented in (Vasudevan, et al., 2009) and here updated with new publications and one additional plant, the pulp mill.

Table 2.3 - Benchmarks for Plantwide Control (Vasudevan, et al., 2009), (updated and reorganized).

\begin{tabular}{|c|c|c|}
\hline Process & Authors (Proposers) & Authors (Appliers) \\
\hline $\begin{array}{l}\text { Hydrodealkylation } \\
\text { (HAD) Plant }\end{array}$ & (Stephanopoulos, 1984) & $\begin{array}{l}\text { (Ponton \& Laing, 1993), (Fonyo, } \\
\text { 1994), (Ng \& Stephanopoulos, } \\
\text { 1996), (Cao \& Rossister, 1997), } \\
\text { (Luyben, et al., 1998), (Luyben, et al., } \\
\text { 1997), (Kookos \& Perkins, 2001), } \\
\text { (Luyben, 2002), (Herrmann, et al., } \\
\text { 2003), (Qiu, et al., 2003), (Bildea \& } \\
\text { Dimian, 2003), (Vasbinder, et al., } \\
\text { 2004), (Konda, et al., 2005), (Konda, } \\
\text { et al., 2006), (Araujo, et al., 2007a), } \\
\text { (Araujo, et al., 2007b), (Bouton \& } \\
\text { Luyben, 2008). }\end{array}$ \\
\hline $\begin{array}{l}\text { Styrene Monomer } \\
\text { Plant }\end{array}$ & (Turkay, et al., 1993) & (Zhu \& Henson, 2002). \\
\hline
\end{tabular}




\begin{tabular}{|c|c|c|}
\hline Process & Authors (Proposers) & Authors (Appliers) \\
\hline $\begin{array}{l}\text { Tennessee } \\
\text { Eastman (TE) } \\
\text { Challenge }\end{array}$ & $\begin{array}{l}\text { (Downs \& Vogel, 1993) } \\
\text { and (Bathelt, et al., 2015) } \\
\text { (revised and extended } \\
\text { version) }\end{array}$ & $\begin{array}{l}\text { (McAvoy \& Ye, 1994), (Price, et al., } \\
\text { 1994), (Lyman \& Georgakis, 1995), } \\
\text { (Ye, et al., 1995), (Ricker \& Lee, } \\
\text { 1995), (Banerjee \& Arkun, 1995), } \\
\text { (Baughman \& Liu, 1995), (Luyben \& } \\
\text { Luyben, 1995), (McAvoy, et al., } \\
\text { 1996), (Ricker, 1996), (Luyben, et } \\
\text { al., 1997), (Luyben, et al., 1998), } \\
\text { (Tyreus, 1998), (McAvoy, 1999), } \\
\text { (Larsson \& Skogestad, 2000), } \\
\text { (Stephanopoulos \& Ng, 2000), } \\
\text { (Kookos \& Perkins, 2001), (Wang \& } \\
\text { McAvoy, 2001), (Chen, et al., } \\
\text { 2003), (Jockenhövel, et al., 2003), } \\
\text { (Cheng, et al., 2004), (Tian \& Hoo, } \\
\text { 2005), (Antelo, et al., 2008), } \\
\text { (Molina, et al., 2011). }\end{array}$ \\
\hline $\begin{array}{l}\text { Vinyl Acetate } \\
\text { Monomer (VAM) } \\
\text { Plant }\end{array}$ & $\begin{array}{l}\text { (Luyben, et al., 1997), } \\
\text { (Luyben, et al., 1998) }\end{array}$ & $\begin{array}{l}\text { (Chen \& McAvoy, 2003), (Olsen, et } \\
\text { al., 2005), (Psaltis, et al., 2014). }\end{array}$ \\
\hline $\begin{array}{l}\text { Vinyl Chloride } \\
\text { Monomer } \\
\text { (VCM) Plant }\end{array}$ & (Groenendijk, et al., 2000) & $\begin{array}{l}\text { (Dimian, et al., 2001), (Seider, et } \\
\text { al., 2004). }\end{array}$ \\
\hline $\begin{array}{l}\text { Dimethyl Ether } \\
\text { (DME) Plant }\end{array}$ & $\begin{array}{l}\text { (Vasbinder \& Hoo, 2003), } \\
(\mathrm{Hoo}, 2010)\end{array}$ & \\
\hline $\begin{array}{l}\text { Tert-Amyl } \\
\text { Methyl Ether } \\
\text { (TAME) Process }\end{array}$ & (Al-Arfaj \& Luyben, 2004) & \\
\hline $\begin{array}{l}\text { Pulp Mill } \\
\text { Benchmark }\end{array}$ & $\begin{array}{l}\text { (Castro \& Doyle, 2002), } \\
\text { (Castro \& Doyle, 2004a), } \\
\text { (Castro \& Doyle, 2004b) }\end{array}$ & $\begin{array}{l}\text { (Mercangöz \& Doyle, 2006), } \\
\text { (Marcangöz \& Doyle, 2008), } \\
\text { (Luppi, et al., 2011), (Luppi, et al., } \\
\text { 2013) }\end{array}$ \\
\hline
\end{tabular}


Of the seven plantwide control methods described in Section 2.3, none specify control tuning methods for the overall plant. The authors who designed plantwide control systems for the benchmarks cited in Table 2.3 had to tune the PID or MPC controllers. These authors applied plantwide control design techniques to define the control structure and used classical tuning methods to adjust the controllers. For example, in the pulp mill case, (Castro \& Doyle, 2002) employed independent PI controllers tuned with internal model control (IMC) rules in the regulatory layer and MPC manually tuned in the supervisory layer. In (Castro \& Doyle, 2004a), the authors used IMC and autorelay rules to tune the PID controllers. Later, (Luppi, et al., 2011) and (Luppi, et al., 2013) used IMC and a modified IMC tuning rule to tune PID controllers. In the Tennessee Eastman (TE) case, (Molina, et al., 2011) used the IMC rules to tune the PID controllers in a designed plantwide control system, and (Tian \& Hoo, 2005) designed and manually tuned an MPC to control the process.

As a final remark, all of the application papers about plantwide control shown in Table 2.3 presented a control structure that was successfully tested for at least one simulated scenario.

\subsection{Important Topics in Plantwide Control}

Many important topics in Plantwide Control are addressed by the specialized literature. The ones most relevant to the challenges of modern processes and to present study are described in this section.

\subsubsection{Control Objectives}

One of the central points in designing a control system is the specification of the control objectives and constraints (Foss, 1973). These will determine what characteristics the control system will try to provide to the process, and their correct selection is crucial to a good design. A broad review of several relevant performance indexes and constraints is presented in (Juliani, 2012).

As a general economic performance function, Zheng et. al. (1999) proposed the use of the plant profit $(P)$, which is defined as the difference between the revenues $(R)$ and sum of the raw materials and utility cost $\left(C_{R U}\right)$, labor costs $\left(C_{\text {Labor }}\right)$ and annualized 
cost for the control system hardware and software $\left(C_{C S}\right)$, as shown in Equation (2.6) (Zheng, et al., 1999), which is employed in many control design procedures.

$$
P=R-C_{R U}-C_{\text {Labor }}-C_{C S}
$$

More generally, Equation (2.7) is a generic performance function, which can be specified to describe any continuous or discrete performance function or constraint as a function of the process states $x_{p}$, controller states $x_{c}$, references $r$ and time, continuous $\left(t_{c}\right)$ or discrete $\left(t_{d}\right)$.

$$
\begin{aligned}
J & =h\left(x_{p}\left(t_{f}\right), x_{c}\left(t_{f}\right), t_{f}\right)+\int_{t_{c_{0}}}^{t_{c_{f}}} g\left(x_{p}\left(t_{c}\right), x_{c}\left(t_{c}\right), r\left(t_{c}\right), t_{c}\right) d t_{c} \\
& +\sum_{t_{d_{0}}} g\left(x_{p}\left(t_{d}\right), x_{c}\left(t_{d}\right), r\left(t_{d}\right), t_{d}\right)
\end{aligned}
$$

It should be noted that even for small plants, a single objective function that describes its optimal operation can be very complex or even unattainable. Instead, process control objectives and constraints are better described by sets of equations, and it is a challenge how to properly deal with these many objectives.

While the majority of plantwide control design techniques employ a single and simplified performance equation as specification for optimality, an approach that can optimize multiple performance indexes and allows a more complete control specification will be developed here.

\subsubsection{Distributed versus Centralized Approaches}

From optimal control theory, it is immediately obvious that a true optimal plantwide controller is a single large multivariable controller. However, such controller is impractical and almost impossible to be designed and tuned for large-scale systems. Such single controller can be applied to simple process, but it is considered that it will never be well-applicable to any standard plant (Gernaey, et al., 2012).

Moreover, unit control and distributed control strategies are easy to understand for operators and engineers, making it simpler to fix the process when something goes wrong, with no need of much expertise in the control (Downs \& Skogestad, 2011). 
Therefore, to provide an optimal and safe plant operation, a good plantwide control system should integrate all the controllers in a single interacting system, but the controllers themselves must be of reduced dimensions. In other words, it is possible to employ controllers that concentrate all variables from a unit, but the use of such big controller can create serious problems and should be avoided.

\subsubsection{Steady-State and Dynamic Approaches}

As observed in (Downs \& Skogestad, 2011), it is usually assumed, for steadystate processes, that the rate of accumulation of each component is zero. However, that must be ensured by the control system and, moreover, the material balance must be maintained locally and globally in steady-state. When the control system is not capable of performing a perfect control, which happens in most situations, the steadystate approach fails to provide optimal process behavior. In other words, while the common notion in process engineering is that the process economics are solely determined by steady-state process design, this does not hold true if the control system is unable to keep the processes at the desired operating point all the time.

In fact, the dynamics caused by disturbances, changes in active constraints, controllability, and process and product variability can have great impact on the process economics and must be taken into account (Foss, 1973), (Downs \& Skogestad, 2011), (Juliani, 2012). Moreover, the process dynamics can greatly affect process economics and it is heavily dependent on the implemented control system (Narraway \& Perkins, 1994). That way, optimal steady-state operation does not imply optimal operation in a real scenario with disturbances (Zheng, et al., 1999).

Finally, although many control design techniques are based on steady-state information, especially the RGA (Relative Gain Array) and its variants, this has been proven not to be a good approach. Such approaches can even be misleading in several cases (Skogestad, et al., 1990) and should be avoided.

\subsubsection{Self-optimizing Control}

The concept of self-optimizing control was first proposed in (Morari, et al., 1980) as "a function c of the process variables which when held constant, leads automatically 
to the optimal adjustments of the manipulated variables". The term was then first introduced by (Skogestad, 2000a) with a revised definition: "self-optimizing control is achieved if a constant setpoint policy results in an acceptable loss (without the need to re-optimize when disturbance occurs)". In other words, a self-optimizing control is one that will always take the process to an optimal state with its setpoint constant, without the need of reoptimization to find new setpoints when a disturbance occurs. A clear discussion of this topic, with several examples, is presented in (Skogestad, 2004b). A recent review on self-optimizing control was presented in (Jäschke, et al., 2017).

The self-optimizing control concept proposed by (Morari, et al., 1980) and (Skogestad, 2000a) is quite promising, but has not been effectively applied in many works. Thus, an open research area would be to further develop the concept of selfoptimizing control and to apply it in practical cases.

\subsubsection{Closed Loops and Degrees of Freedom}

Theorem (Larsson \& Skogestad, 1998). "The closing of a lower-layer (partial) control system, involving the manipulated input $u_{2}$ and the measured and controlled variable $y_{2}$, introduces no new control limitations (e.g. in terms of right half plane zeros) provided:

1. The setpoints $y_{2_{s}}$ (for $y_{2}$ ) are available as degrees of freedom at the next layer;

2. The measurements $y_{2}$ are available at the next layer;

3. The controller interconnecting $y_{2}$ and $u_{2}$ is minimum phase and stable (but may have integrators)."

More generally, a controller $c_{1}$ will introduce no limitation to the actions of higher layers in the process if it is possible to generate a filtered setpoint $f(r(t))$ so that the controller manipulated variable $u(t)$ will follow the original setpoint $r(t)$, as described by Equation (2.8) and illustrated in Figure 2.3.

$$
\left\{\begin{array}{c}
u(t)=c_{1}(f(r(t))) \\
u(t)=r(t)
\end{array}\right.
$$




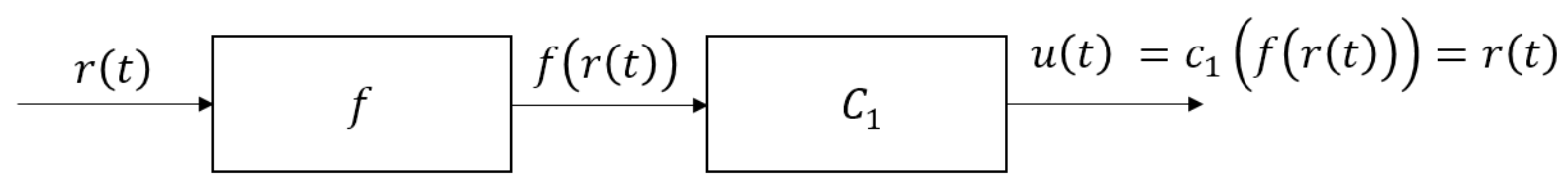

Figure 2.3 - Non-limiting controller.

It should be noted that $c_{1}$ does not need to be invertible or linear, it is only needed that a signal $f(r(t))$ can be obtainable for the desired references $r(t)$ such that $c_{1}(f(r(t)))=r(t)$.

This is an important remark to consider when designing a multilayer control system, where different layers take care of different control tasks, but should not limit each other if the system is properly designed.

This theorem should be applied to multilayer control design procedures to guarantee that an over-restrained lower level control does not limit the action of a higher-level controller. For example, Skogestad's Seven-Step Plantwide Control Procedure results in a multilayer control system and the regulatory controllers should respect the theorem's premises in order to allow the supervisory control to optimize trajectories without being limited by the regulatory level constraints.

\subsubsection{Selection of Controlled Variables}

In a given control structure, two errors are present: the setpoint error and the implementation error. The setpoint error is defined as the difference between the setpoint and its global optimal value, while the implementation error is the difference between the process variable value and its respective setpoint. The former can be caused by changes in the operating point of the process, while the latter is due to measurement error and poor control. Considering this, a good candidate for controlled variable must satisfy the following requirements (Skogestad, 2000a) :

1. Its optimal value should be insensitive to disturbances (i.e., the control should present a near-optimal performance without the need of constant reoptimization);

2. It should be easy to measure and control accurately; 
3. It should be sensitive to changes in the manipulated variables, that is, the gain from the manipulated variables to the controlled variable should be large;

4. For cases with two or more controlled variables, the selected variables should not be closely correlated.

Some quantitative methods to determine controlled variables are presented in (Halvorsen, et al., 2003), (Skogestad, 2000a), (Larsson, et al., 2001).

A major decision to be taken in the design of a control system is the choice of the process variables to be controlled and most plantwide control design procedures include a step to make this choice. However, how to choose such controlled variables is still an open research topic and well-defined techniques to make this decision need to be developed.

\subsubsection{Control of Recycling Systems}

Control of cascade systems is widely present in the literature and commonly used in the industry. Processes with recycles, although very common, do not have so many control techniques available, rather, the typical approach to this problem is to use large surge tanks to decouple the recycling dynamics and turn the process into an almost-non-recycling one, which can be a very expensive solution, with impact in operation performance and process safety (Luyben, 1993) and also increases delays and time constants due to the longer residence times.

Processes with recycles need to receive special attention when designing a control system, "since the recycle can change the process dynamics in a way that may not be apparent from the dynamics of the individual unit operations" (Konda, et al., 2005). Furthermore, processes with recycle are prone to the "snowball effect", defined in (Luyben, 1994) as: "a small change in a load variable causes a very large change in the recycle flow rate around the system", which can be a consequence of an unsatisfactory flow control in a recycle stream. This effect is also discussed in (Luyben \& Luyben, 1995), (Luyben, et al., 1998) and (Seider, et al., 2004).

Although the changes in dynamics caused by closing loops are widely studied in Control Engineering, this matter still needs to be well addressed in Plantwide Control. 


\subsubsection{Control of Unstable Units}

Unstable loops are very common in a wide variety of processes. Stabilizing them can be a primary control specification, but these variables can be part of a larger system to be controlled.

When taking the steady-state approach in processes with integrators or other unstable loops, the usual approach is to first close and stabilize these loops and then perform a steady-state analysis, even if dynamics are taken into consideration. This approach, however, is sub-optimal and should be properly addressed (Arkun \& Downs, 1990).

By simply stabilizing unstable loops before starting the proper plantwide control design, these stabilizing controllers early uses degrees of freedom and become new constraints to the problem, which can deviate the final control performance from its actual optimal behavior. To avoid that, a good design technique should properly address these variables with all others, considering the stabilization of such variables as part of the design objectives, instead of firstly stabilizing them with the available manipulated variables.

\subsubsection{Process Modeling}

Process modeling can be a very important and time-consuming task in the design of control systems (Foss, 1973). However, the authors of the previously cited methods do not refer neither to the kind of models that their methods require (e.g.: rigorous phenomenological models, identified models, low order models obtained by steps) or to the accuracy expected from such models. What is normally mentioned is if the models are linear or nonlinear. Thus, a clearer specification of the desired model is a topic that should be addressed in plantwide control design procedures.

A model is a representation of a system, it can be presented in a wide variety of forms and each can be obtained from different ways. The most suitable model representation and modeling technique for control design depends on the controller being designed, the design technique being applied, the target process, the control specifications, the available data and a series of other factors. 
The main model representations available for plantwide control design in its various stages are: graphical models (Ogata, 2009), impulse response, step response, frequency response (Ogata, 2009), transfer functions (Aguirre, 2007), polynomial models (Ljung, 1999), neural networks (Haykin, 2009), state space representation (Monteiro, 2006), (Hespanha, 2009) and computational models, which are computational simulators built with the other cited representations. A concise review of such representations is presented in (Juliani, 2012).

As for the modeling techniques, there is the theoretical modeling (Garcia, 2005) and the system identification (Ljung, 1999), (Aguirre, 2007) approaches. The first approach is the construction of a model applying the physical knowledge of the process behavior, while the second one is the estimation of a model from process data.

It is important to notice that there is no single best representation of a process, but a most suitable one for each task. Considering that it is possible to conclude that the usual approach of finding a good process model and then performing the complete plantwide control design with such model can provide a good result, but a better one can be achieved if, for each task of the design procedure, the most adequate model is employed. This will be further explored in the next chapters.

\subsubsection{Design of the Plantwide Control System: Before or After the Plant is Built?}

A topic that is not normally addressed in the plantwide control literature is whether the design of the control system should be performed after the plant is built or simultaneously with the process design. This is a topic that should be addressed in future researches and could be of particular importance to increase the number of practical applications of the plantwide control design procedures.

If the plant is under design, the control design builder will certainly need a reliable heuristic plantwide approach that is not based on a precise process model, as considered, for instance, in Luyben's Nine-Step procedure (Luyben, et al., 1997), (Luyben, et al., 1998). However, if the plant is under design, this presents some advantages, such as the possibility to insert new sensors and actuators or to reallocate them (to avoid long dead times, for instance). If the plant is built and operating or if the 
process synthesis has been completed and a dynamic simulator is available, a precise mathematical model can be obtained for the process, which can be used in mathematical-oriented approaches, such as the Integrated Framework of Simulation and Heuristics method. Nonetheless, when the plant is already operating, adding new or relocating the existing instruments may not be viable.

\subsection{Concluding Remarks}

This chapter presented a review of Plantwide Control, focusing on a general presentation of the topic, with the highlighting of the most relevant publications and the main open issues. The major architectures were presented and briefly commented, followed by the enumeration of plantwide control design techniques, with the major ones briefly described. Benchmarks employed in Plantwide Control were also identified.

A close study of the available plantwide design techniques reveals that the majority of them focus on small and midscale processes and they are only adequate for the selection of control structures of process units. When it comes to designing control systems for a complete process or a large-scale system, only a few published techniques are applicable, mainly the ones described in Section 2.3. This fact shows that there are still many opportunities of improvement in this matter and corroborates the need of new general techniques that can be applied to large processes.

In regard to the cited plantwide control design techniques, it is also possible to note that most of them aim to design a functional and operator-friendly control system. Optimal operation is only primarily sought by a few recent techniques, and mostly in a partial way. This demonstrates the need of a plantwide design technique that not only provides an operational and operator-friendly control system, but also enhances optimality and robustness of the process.

Section 2.5 briefly details some of the main research opportunities in Plantwide Control to be solved in the near future and that can greatly enhance the practical use of plantwide control design techniques. 
Research in Plantwide Control has had advances in the recent years, but there are still many gaps needing improvements to spread its application in the industry.

When the control objectives are analyzed, it can be observed that the recent design techniques tend to include economical functions as main objectives. However, the procedures are limited to a single and simplified objective function. An opportunity of overall performance improvement and enhancement of the specifications flexibility lies in the development of a design technique that allows the use of multiple objective functions to be optimized or used as constraints.

Analyzing the use of process models in the various design techniques, it is noticeable that the approaches usually seek the minimal use of process models or are based on a single supposedly perfect model. However, from System Identification, it is known that multiple models can better describe a process, each focused on a different aspect or with a certain dimension and uncertainty. Therefore, a technique that makes use of this fact can provide better results when, at the same time, would be able to use simpler models for some decisions and more precise and costly models for other decisions, according to the availability and feasibility of each model.

Finally, as stated by the Plantwide Control Design definition, controller design and tuning is commonly handled after the control system is designed. However, the choice of the best control structure and type is dependent on the final controller and its tuning, which are also dependent on the available process information (Juliani, 2012). Thus, improvements in the final control system quality can be achieved if the controllers' design and tuning are included in the plantwide design procedure.

These opportunities will be explored in this study and tools to address them and other plantwide control issues will be presented in the next chapter. 


\section{Theoretical Tools for an Augmented Plantwide Control Design Technique}

With the previous works and current open issues of Plantwide Control Design in mind, some theoretical tools are presented in this chapter to be used further ahead in the proposition of an augmented plantwide control design technique.

First, some considerations about the use and the impact of System Identification in plantwide control design are made. Next, the concept of optimality is defined and its application to plantwide control is discussed. Then, Bellman's principle of optimality is presented in the context of plantwide control for assuring plantwide optimality over local optimality in the final control system. Next, the concepts of Nash equilibrium and Pareto optimality are presented. A discussion regarding the cooperation of multiple independent controllers follows. Then, a technique for control optimal tuning is presented. Finally, multi-objective optimization is discussed, some solution techniques presented and a new augmented lexicographic approach proposed.

\subsection{System Identification for Large Scale Systems}

When obtaining models for model-based control design, phenomenological modeling can be used to some extent, but the use of system identification will be more practical, and needed, for most cases, especially for complex and large systems.

System Identification is well established for simple and small processes, but still has many open research topics for large and complex systems. When the identification experiments are taken into consideration, another important matter that arises is that highly informative experiments are only performable for some variables simultaneously and identifying a complete large system at once can cause many issues, such as keeping the process in safe operating regions and avoiding unstable modes, being it almost impractical to perform. Thus, regardless of the identification approach employed, a plantwide model can only be obtained through several small to mid-scale identification experiments and this can be an expensive and very time-consuming task, as widely stated in the literature. 
Therefore, a good plantwide control design technique should be able to rely on partial and multiple models. Moreover, system identification should be included as part of the plantwide control design procedure, instead of being considered a previously concluded task. This approach can result in several benefits, specifically:

$>\quad$ It will be possible to specify the objectives for system identification according to the necessities of the plantwide procedure, instead of it simply being "to obtain a good process model";

$>\quad$ The identification experiments can be planned to necessity, minimizing their cost and total length, while optimizing the model quality;

$>\quad$ The plantwide procedure steps can be adapted to the available models, instead of requiring a specific model type and quality;

$>\quad$ The system identification can be performed progressively across the various design steps, according to the needs, instead of being performed all at once before the design procedure; and

> All decisions and design steps performed using identified models will be based on more precise dynamic information obtained from the process and the final implemented results will be closer to the intended during the design.

For example, at an initial step of the plantwide control design procedure, an expected model or a model derived from historical data or from similar plants can be used. Later on, a simple model focused on low-frequency band can be identified in small blocks, with the variable groupings made according to the groupings chosen for control. Finally, more detailed models for final decisions and control tuning can be identified for groups of variables and with some controllers already in place, such as stabilizing controllers.

Considering the ideas exposed, while most of the plantwide control techniques suppose that a model is initially available, a new approach that includes system identification as one of its tasks can provide some important advantages.

\subsection{Optimality in Plantwide Control}

Any control system that aims to be optimal in some way needs to optimize a performance function or a set of functions in the form of Equation (2.7). 
Besides the performance function itself, two aspects should also be considered, its sensitivity to process variables and its robustness to uncertainties.

\subsubsection{Optimality Sensitivity}

Sensitivity of a performance function to a process variable is defined as the intensity of changes in this function value when there is a change in the process variable or, mathematically, as the partial derivative of the performance function to the process variable.

This concept is employed in many techniques of selection of manipulated variables, controlled variables and performance functions. Some guidelines related to the sensitivity of a performance function should be observed:

A A performance function should be sensible to all its variables:

- If a variable does not influence significantly a performance function, it should not be included in this function in order to decrease the system's complexity and reduce computational efforts;

- It is important to notice that, in some cases, the performance function is sensible to certain variables in only certain process scenarios, which still make these variables relevant to the performance function.

$>\quad$ When building a controller to indirectly optimize a performance function (i.e., the function is not explicitly included in the controller), the variables to which the function is most sensible are good candidates for controlled and manipulated variables; and

$>$ If a performance function is sensible to a disturbance, the effects of this disturbance should be compensated by a controller.

\subsubsection{Optimality Robustness}

The concept of optimality robustness describes to how the optimal value of a performance function changes face to uncertainties in the model used to build a control system. If a system has good optimality robustness, the performance of the implemented control system will remain optimal even if there are errors in the models used to design the control system (Juliani, 2012). 
It is very important to include the optimality robustness in the design and tuning of a control system, since the models available are always subject to uncertainties, structured and unstructured (Juliani, 2012).

Finally, it is preferable a control system with worse nominal optimality and better optimality robustness than the opposite. Quantitatively, however, this trade-off needs to be specified appropriately for a good overall control.

An objective discussion about optimality robustness and robustness in general is presented in (Juliani, 2012).

\subsection{Bellman's Principle of Optimality and Plantwide Control}

In this section, general optimality in plantwide control is studied. For that, a simple example with recycle is presented, followed by the description of Bellman's Principle of Optimality and a discussion of how it can be applied to enhance optimality in plantwide control.

\subsubsection{Example Scenario with Recycle}

Consider a simple process with a recycle, in which a controller $(A C)$ controls a composition (measured by AT) in a reactor, through the manipulation of the feed flow (regulated by FC-1 through the valve FV-1) and the recycle flow (regulated by FC-2 through the valve FV-2), as shown in Figure 3.1.

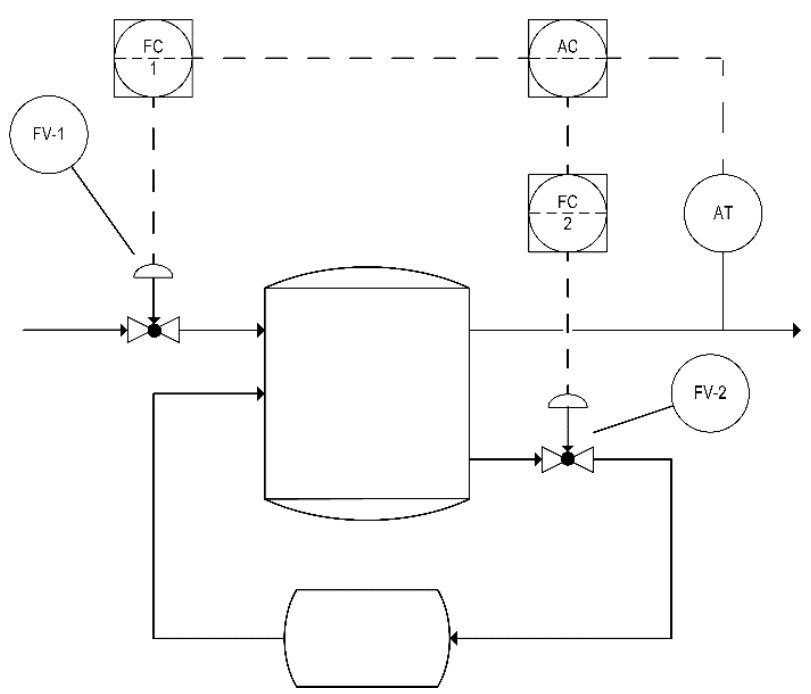

Figure 3.1 - A simple process with recycle. 
Consider this system controlled by an ideal Model Predictive Controller (MPC) built with the main process model $G$, which describes the direct effect of FV-1 and FV2 on AT, but has no explicit information about the recycle.

Suppose the occurrence of a large disturbance in the feed input. To keep the output in desired conditions, the controller acts on both manipulated variables in an optimal way, reducing the feed flow and increasing the recycling flow to compensate the disturbance. If the recycling dynamics are negligible, this does correspond to a global optimal behavior and the process will move back to the optimal operating point.

However, if the recycling dynamics are significant, the disturbance will return as a new disturbance to the process, which will be optimally rejected by the controller, by further increasing the recycling flow, triggering the so-called snowball effect (Luyben \& Luyben, 1995), (Luyben, et al., 1998). In this scenario, the controller continues to pursue an optimal response, but the snowball effect on the recycle takes the whole process away from its optimal operating point.

For this problem, the usual approach is to have a controller in the main process and to install a surge tank on the recycle to slow the recycling dynamics. This, however, is not optimal. A better solution would be to employ a controller with a different structure.

A first candidate for a better controller is a multivariable controller that includes the recycling dynamics. While this is a very good solution for simple processes, the recycle can include other units and many variables, cases in which a very large controller would be needed. In these cases, however, it is not a generally viable solution due to the various factors previously mentioned.

The resulting question from this discussion is then how to specify a control system that is optimal for the whole process.

\subsubsection{Bellman's Principle of Optimality}

"An optimal policy has the property that whatever the initial state and initial decisions are, the remaining decisions must constitute an optimal policy with regard to 
the state resulting from the first decision" (Bellman, 1957). This is known as Bellman's Principle of Optimality.

The principle of optimality can enhance the process of decision-making in the following manner (Kirk, 1970):

Consider a process in a state $b$ and the allowable decisions that can take the process to the states $c, d$ or $e$ in the next step with a cost of, respectively, $J_{b c}, J_{b d}$ and $J_{b e}$. From each of these states, the system can be taken to a desired final state $f$ with optimal costs $J_{c f}^{*}, J_{d f}^{*}$ and $J_{e f}^{*}$. The principle of optimality implies that, if $b-c$ is the initial step, then $c-f$ is the subsequent optimal path to $f$, with the analogous concept valid for the other states. So, the optimal path from $b$ to $f$ can be found by the minimal cost of the set (3.1).

$$
\left\{\begin{array}{l}
C_{b c f}^{*}=J_{b c}+J_{c f}^{*} \\
C_{b d f}^{*}=J_{b d}+J_{d f}^{*} \\
C_{b e f}^{*}=J_{b e}+J_{e f}^{*}
\end{array}\right.
$$

This concept is employed in dynamic programming, and can be extended to plantwide control to enhance controller specifications and, consequently, the plant behavior, as the discussion that follows.

\subsubsection{Ensuring Optimality in Plantwide Control}

If the principle of optimality is applied to the described scenario, the considered controller is locally optimal for its objective function and model by taking control actions to optimally take the process from any state to the targeted optimal state.

However, when the complete system is considered, the control is suboptimal. This is easy to verify if it is considered that, in the final steady state (supposing no new disturbances occur), the system should be back to the target state, with this achieved by a series of control actions that use the recycle, but keeps it manageable. However, if the controller has no information about the recycle, it will reject the disturbance in a local optimal manner, increasing the recycle flow and initiating the snowball effect.

Finally, to ensure optimality to the complete process, the control system must follow Bellman's Principle of Optimality in a general dimension, and not only locally. In 
other words, it must be ensured that, for every local control action taken, the future trajectory of the complete process states is not taken away from its optimal path.

\subsection{Nash Equilibrium and Pareto Optimality}

In a multi-agent system, the Nash equilibrium is defined as the stable equilibrium in which local optimality is achieved by each local agent (Rawlings \& Steward, 2008).

A Pareto set, or Pareto front, is the set of Pareto optimal solutions of a multiobjective problem. A Pareto optimal solution is such that an improvement in any objective function will result in a loss in at least one other objective function.

All Pareto optimal solutions are a Nash equilibrium, however, not all Nash equilibria are in the Pareto front. In fact, for dynamic systems, Nash equilibrium can be stable and close to the Pareto front, stable but far from the Pareto front or unstable (Rawlings \& Steward, 2008).

In a process controlled by decentralized controllers, the system will be attracted to a Nash equilibrium, in which each controller is locally optimal. However, this does not imply that the system is Pareto optimal, i.e., there may be another control configuration, with another Nash equilibrium, for which at least one objective function is improved without implying any loss in the other objective functions.

The important conclusion from this discussion is that a control system in which each controller optimizes a local objective function (a Nash equilibrium) does not guarantee overall optimality, and that cooperation must be enforced between the controllers to achieve an overall optimality (a Pareto optimal solution).

\subsection{Cooperation between Multiple Controllers}

This section focuses on the problem of the cooperation of multiple controllers acting on the same process. For that, a simple example scenario is described next.

Consider a process with multiple stages, each with an independent controller, as shown in Figure 3.2. 


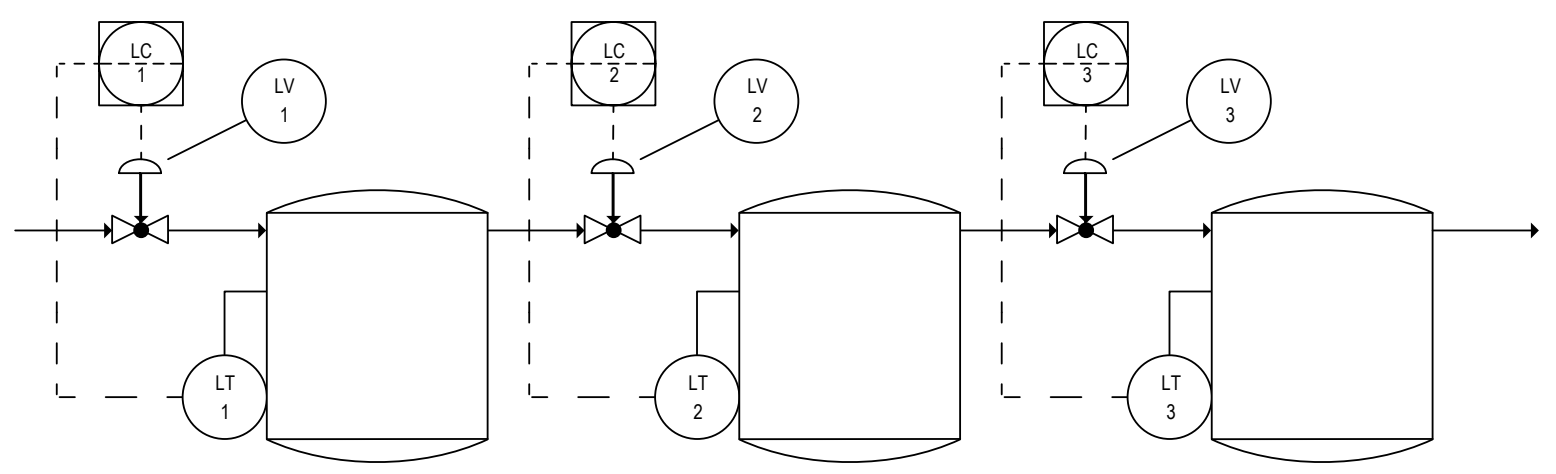

Figure 3.2 - A simple process with multiple controllers.

In this process, each level controller (LC-1, LC-2 and LC-3) regulates the level, measured by a transmitter (LT-1, LT-2 and LT-3) in the associated vessel, by the manipulation of the respective feed flow (regulated by the valves LV-1, LV-2 and LV3). In addition, other controllers can be present on the process as, for example, analytical regulators, in such a way that the level in each reactor can be a secondary controlled variable.

A scenario is then supposed, in which a strong disturbance occurs in the level of the final reactor, which can be caused, for example, by a sudden reduction in the output flow. If the level controllers have a tight tuning, the input flow regulated by LV-3 will be reduced and the disturbance will be propagated to the second reactor. Then, LC-2 will reduce the flow through LV-2 and the disturbance will be further propagated to the first reactor. Until the disturbance is fully compensated, it will continue to be propagated upstream, which is, in most cases, an undesired phenomenon. If the control structure is changed and the output flows are used to regulate the tanks levels, the same disturbance propagation will occur for a strong disturbance entering the first tank.

The use of a loose tuning for the level controllers could mitigate this propagation phenomenon, but there can always be a disturbance strong enough to trigger the propagation, which makes the definition of optimal looseness quite imprecise. Furthermore, this is not a viable solution for every process variables. A possible direct solution would be to employ a single large controller that regulates all variables in an optimal way, but this is not, as previously stressed, a generally viable solution. A better control structure is then desired. 
This discussion can be extended to several other cases with multiple controllers, in which, due to the interaction between loops, the action of one of them can be prejudicial to the others. Or, even worse, when trying to be independently locally optimal, the various controllers start to compete and trigger phenomena that can be very prejudicial to the system as a whole, such as the propagation of disturbances and oscillations.

It is then clear that simply to optimize independently the units of a plant does not consist in a plantwide optimal strategy and that cooperation between controllers and plantwide optimality needs to be included in the plantwide control design.

The resulting question from this discussion is how to ensure cooperation among controllers to achieve an optimal plantwide behavior in a system with multiple controllers.

A first approach to enforce cooperation between controllers is to use an hierarchical control architecture, in which the higher layers employ multivariable controllers that coordinate the lower layers through their setpoints while the lower layers deal with fast dynamics, simplifying the dynamic problem to be solved by the higher layers of controllers.

A second solution to this problem is to tune the independent controllers in a multi-loop approach, instead of independently. This way, the complete system is considered in the tuning of each controller and destabilizing and oscillatory competitions between controllers are avoided.

These two suggested approaches can be used together to seek a plantwide optimality, as will be done in the plantwide control design technique proposed in this work.

Other solutions are also possible, such as the use of complementary variables to adjust control constraints, communication-based controllers, or cooperation-based controllers (Rawlings \& Steward, 2008). Since these approaches are still under development and are not yet widely employed in the industry, they will not be addressed in the present work, but are interesting topics for future research. 


\subsection{Multi-Objective Optimization}

Plantwide control design is intrinsically a multi-objective problem, i.e., there are multiple objectives that are desired to be optimized, some of them conflicting and some of them unrelated, and it is a design challenge to choose which Pareto optimal solution should be chosen in detriment of others. Multi-objective optimization problems also arise in many other areas in which there are conflicting objectives to be optimized, such as optimal control tuning (Juliani, 2012).

This section describes the multi-objective optimization problem, some solution approaches, and its application to plantwide control design.

\subsubsection{The Multi-Objective Optimization Problem}

Classical optimization theory and practice deal with the optimization of a single objective function subject to a set of constraints. In an optimization problem, if there is a single objective, there is a single optimal solution or a set of equivalent solutions, i.e., solutions that result in the same value for the objective function. When there are multiple conflicting objective functions to be optimized, a multi-objective optimization problem is characterized and can result in multiple different solutions.

A multi-objective problem can be formally represented as in (3.2).

subject to:

$$
P=\arg \min _{\mathrm{x}} j(x)=\left\{j_{1}(x), j_{2}(x), \cdots, j_{n}(x)\right\}
$$

$$
\begin{aligned}
& h(x)=0 \\
& g(x) \leq 0 \\
& x \in \mathcal{X}
\end{aligned}
$$

in which $P$ is the Pareto set, $j(x)$ is the set of the independent objective functions $j_{i}(x)$, $h(x)$ and $g(x)$ are the problem constraints and $x$ is the optimization domain.

Many problems can be modeled as a multi-objective optimization problem, which can be solved to obtain a Pareto set. For many applications, such as the plantwide control, a Pareto set is not a satisfactory solution, since it is a set of many possible solutions and a single solution from this set must be chosen. The problem that then arises is the choice of a single best solution from the Pareto set. 


\subsubsection{Choice of the Best Solution in a Multi-Objective Optimization Problem}

With the multi-objective problem well formulated, the problem of choosing a single best solution from the resulting Pareto set is here focused.

Finding the optimal value of an objective function can be a challenging problem, but it is mathematically a well-defined problem, i.e., the solution can be infeasible, unique or a set of equivalent solutions (multiple feasible solutions with the same value for the objective function). Choosing a solution from a Pareto set, however, can be a subjective problem instead of a pure mathematical problem.

Approaches for choosing a solution can be categorized into scalarizing, nopreference, a priori, posteriori, interactive and hybrid approaches. A general review on these approaches and their application is presented in (Marler \& Arora, 2004).

The following methods were considered suitable for plantwide control design and are detailed next: composite function methods (present in scalarizing, nopreference and a priori approaches), goal programming and lexographic multiobjective optimization.

\subsubsection{Composite Function Methods}

The most common approach to multi-objective problems is to employ a composite function of the various objective functions, converting them into a single objective function that can be optimized as a classical single-objective optimization problem. Some examples of composite functions are weighted sums, $L_{p}$ norms and utility functions. Equation (3.3) expresses the general composition function for scalarizing (convert a vector into a scalar) multiple objective functions.

$$
f_{c}(x)=c\left(f_{1}(x), f_{2}(x), \cdots, f_{n}(x)\right)
$$

While this is a very straightforward approach, finding an appropriate composite function can be a very difficult problem, especially if the objective functions are of different nature, such as performance functions in different engineering units. 


\subsubsection{Goal Programming}

Goal programming is a classical multi-objective optimization solution technique. It consists in setting a goal solution and then solving a new optimization problem that minimizes the error between this ideal solution and a feasible solution.

The first step in formulating a goal programming problem is specifying a goal solution, which is a desirable solution and not necessarily a feasible solution. A good candidate goal is the ideal solution, which is the point in the objective space at which each objective function takes its optimal value if optimized individually (Jones \& Tamiz, 2010). Next, a new optimization problem is formulated to minimize the error between each objective function and the goal solution, which results in a new multi-objective problem with the same dimensions of the original problem.

This new problem must then be solved by composite function, lexicographic or other multi-objective methods. The advantage of this reformulation is that the new problem is easier to treat than the original one, especially if some normalization is applied to the errors (Jones \& Tamiz, 2010). Moreover, this approach allows an easy measurement of how far any candidate solution is from the ideal solution.

Goal programming was first proposed in (Charnes, et al., 1955) and formalized in (Charnes \& Cooper, 1961). Some major contributions were later introduced in (Ijiri, 1965), (Lee, 1972) and (Ignizio, 1976), followed by an increasing number of publications applying goal programming, as presented in Figure 3.3. A recent review on the topic is presented in (Jones \& Tamiz, 2010).

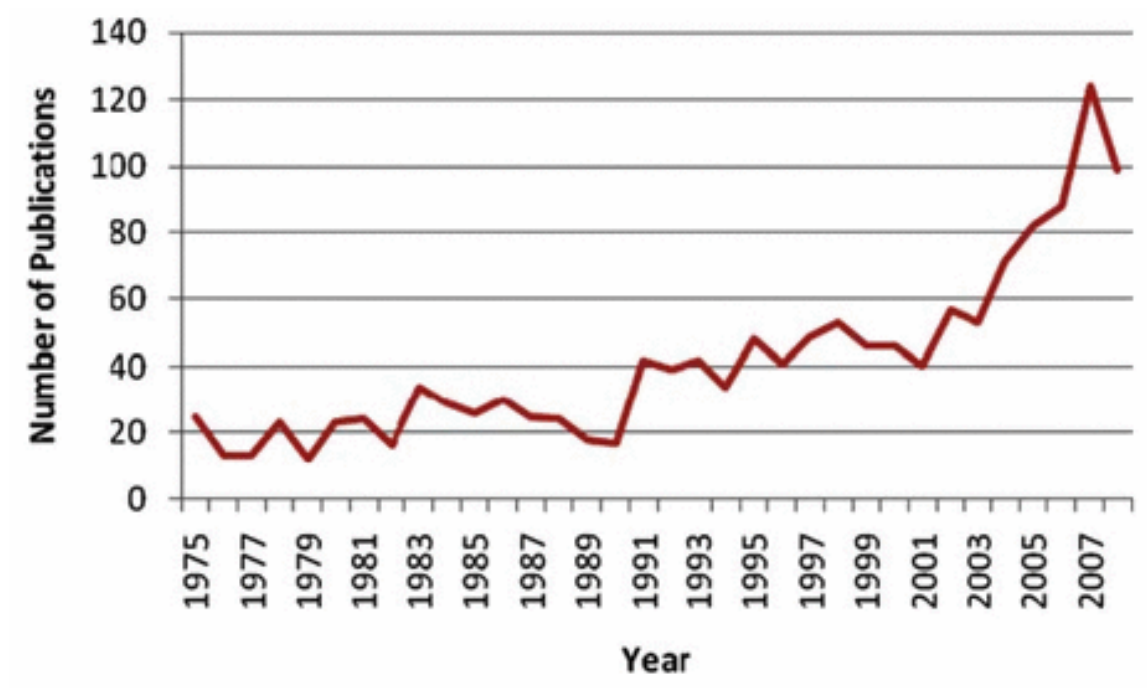

Figure 3.3 - Goal programming publications in the period 1975-2008 (Jones \& Tamiz, 2010). 


\subsubsection{Augmented Lexicographic Multi-Objective Optimization Procedure}

A lexicographic multi-objective optimization offers a more direct way of solving a multi-objective optimization problem. In this approach, the objectives are ranked in order of importance and then the problem is solved as a sequence of single-objective optimizations in the form (3.4).

subject to:

$$
x^{*}=\arg \min _{\mathrm{x}} j_{i}(x)
$$

$$
\begin{aligned}
& j_{k}(x) \leq j_{k}^{*}, \quad k=1, \cdots, i-1 \\
& h(x)=0 \\
& g(x) \leq 0 \\
& x \in \mathcal{X}
\end{aligned}
$$

in which $x^{*}$ is the final optimal solution, $j_{l}(x)$ are the independent objectives, $j_{k}^{*}$ is the value of $j_{k}(x)$ for the step $i=k, h(x)$ and $g(x)$ are the problem constraints and $x$ is the optimization domain.

Therefore, the lexicographic approach finds an optimal solution prioritizing the most important objective functions and optimizing the less important ones without causing any loss to the more important ones.

An augmented lexicographic multi-objective optimization is proposed in (3.5).

subject to:

$$
x^{*}=\arg \min _{\mathrm{x}} j_{i}(x)
$$

$$
\begin{aligned}
& j_{k}(x) \leq \alpha_{k} \cdot j_{k}^{*}+\delta_{k}, \quad k=1, \cdots, i-1 \\
& \alpha_{k} \cdot j_{k}^{*}+\delta_{k} \geq j_{k}^{*} \\
& h(x)=0 \\
& g(x) \leq 0 \\
& x \in X
\end{aligned}
$$

in which $x^{*}$ is the final optimal solution, $j_{l}(x)$ are the independent objectives, $j_{k}^{*}$ is the value of $j_{k}(x)$ for the step $i=k, \alpha_{k}$ is a relative acceptable deviation, $\delta_{k}$ is an absolute acceptable deviation, $h(x)$ and $g(x)$ are the problem constraints and $\mathcal{X}$ is the optimization domain.

In this formulation, each objective function is optimized keeping the more important objectives close to their optimal values, according to a desired precision and following a chosen prioritization order. The introduction of this acceptable deviation improves the quality of the final solution by making the problem more flexible. 
Compared with the classical approach, while the introduction of equality constraints consumes degrees of freedom of the problem, the introduction of acceptable deviation does not, allowing small deviations in some objectives if that leads to gains in others. This is an interesting feature for applications in which small losses in an objective function (even if a more important one) can lead to significant gains in other objectives.

Another important characteristic of this approach is that, if the problem in the first step is feasible, all other steps and the complete problem are also feasible.

The first versions of this augmented approach were applied and successfully tested in control tuning in (Juliani, 2012), (Juliani \& Garcia, 2012) and (Juliani \& Garcia, 2014) and in system identification in (Alves, et al., 2012a), (Alves, et al., 2012b), (Alves, et al., 2013) and (Juliani, et al., 2013).

The augmented lexicographic multi-objective optimization can be described as an algorithm, as shown in Figure 3.4.

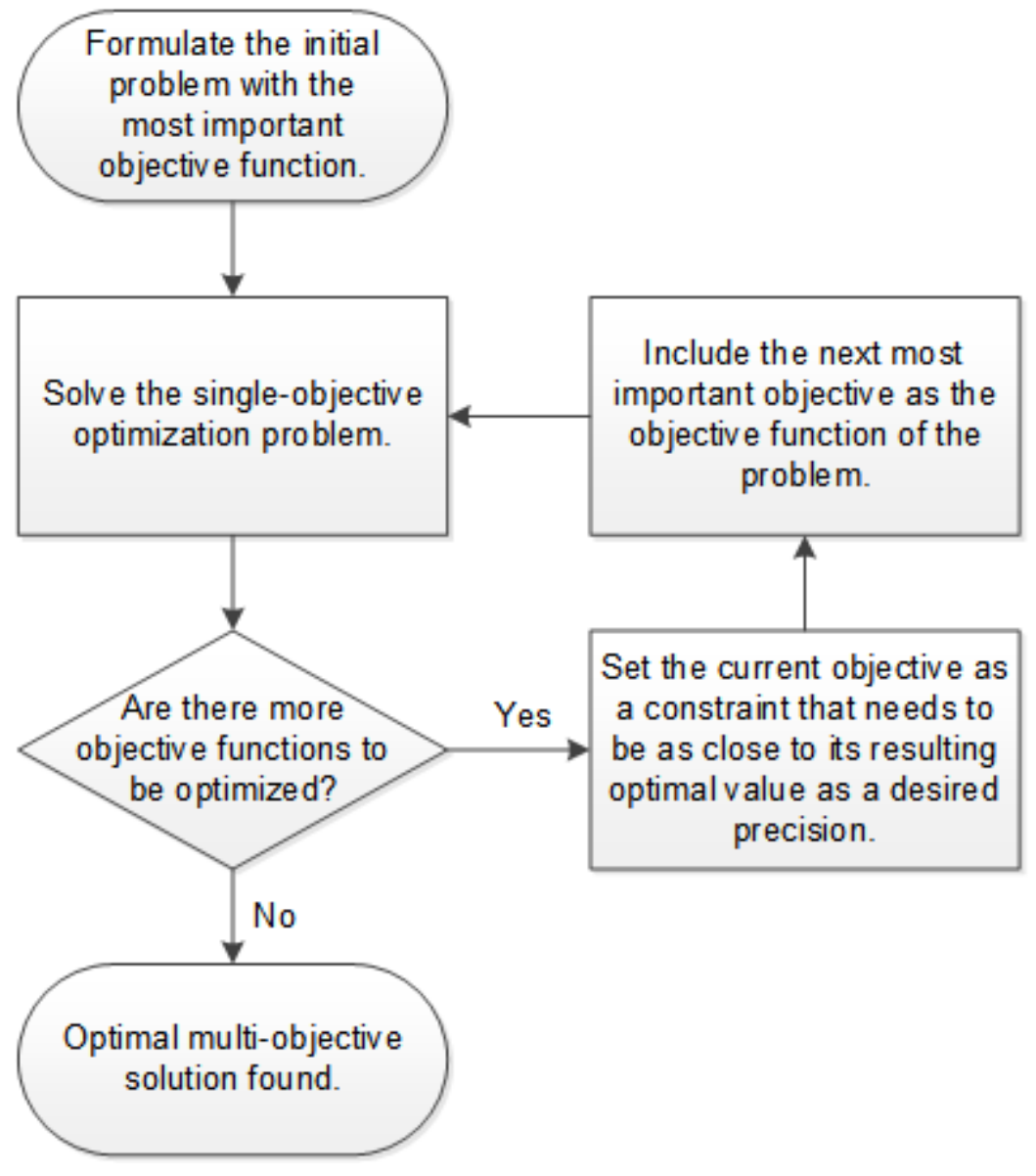

Figure 3.4 - Augmented Lexicographic Multi-Objective Optimization. 
The augmented lexicographic optimization has the benefit of providing a solution that is Pareto optimal without evaluating the whole Pareto set and allowing an easy measurement control of the multi-objective trade-offs.

Compared with the goal programming approach, the lexicographic multiobjective optimization does not employ an infeasible explicit ideal solution as reference, but starts the problem from a Pareto optimal solution and introduces and optimizes a new dimension in the Pareto set at each step. This can be a very appealing feature when optimizing multiple objectives of different nature, as occurs in plantwide control design. This approach also reduces the total number of evaluations of the objective function, once the ideal solution does not need to be calculated, which decreases the computational effort needed to solve the optimization problem.

It is important to stress that, for both classical and augmented lexicographic procedures, the Pareto set is not estimated, and the optimization starts from a Paretooptimal solution and navigates the set to new Pareto optimal solutions as new objectives are optimized.

\subsubsection{Approaches Comparison}

Some of the concepts exposed in this section are illustrated in Figure 3.5.

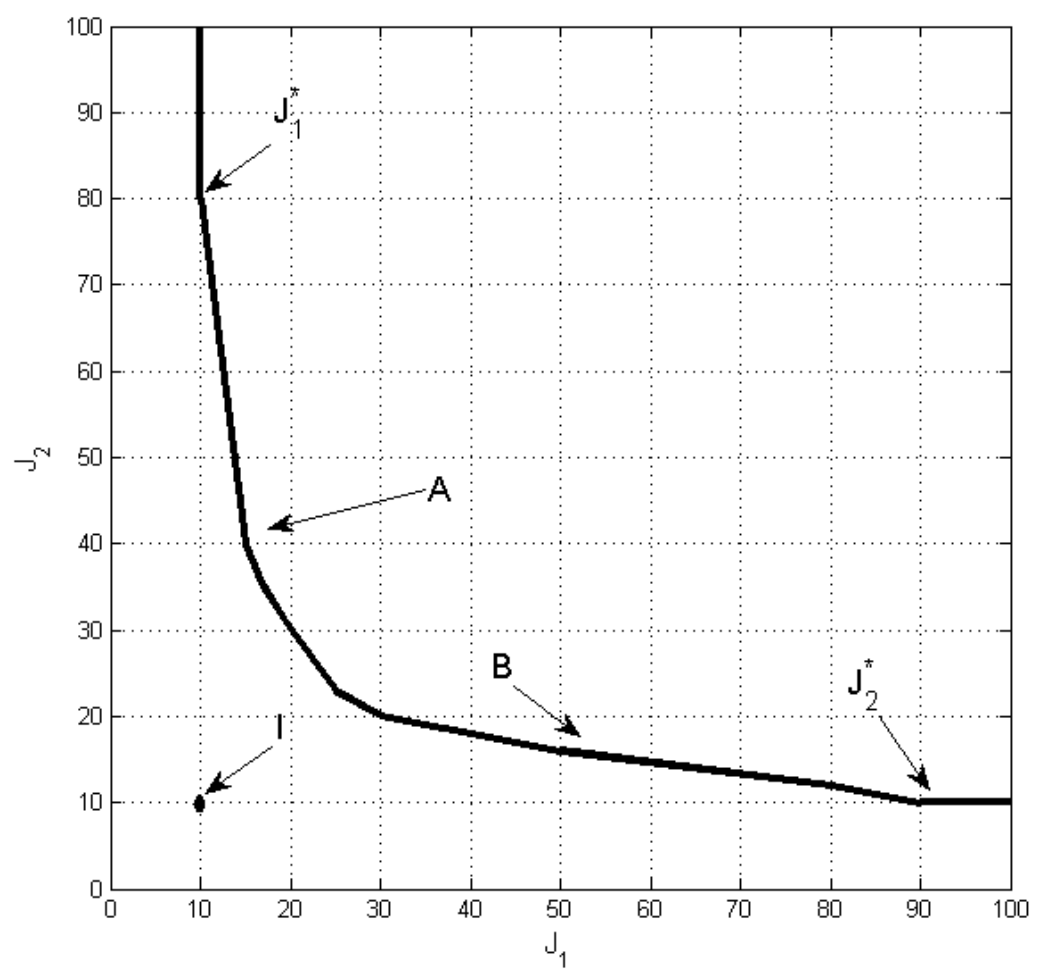

Figure 3.5 - Pareto front. 
In Figure 3.5, the line presents a Pareto set of a multi-objective optimization of the objective functions $J_{1}$ and $J_{2}$, whose independent optimal values are $J_{1}^{*}$ and $J_{2}^{*}$, respectively, and $I$ is the ideal solution for the problem, corresponding to both objectives at their optimal values, which is an unfeasible solution. Furthermore, $J_{1}^{*}$ determines the limit of the Pareto set for which $J_{2}$ can be improved without any loss to $J_{1}$, and $J_{2}^{*}$ limits the same region for the objective $J_{2}$.

If a composite function method is employed to choose a single solution, then a new objective function that scalarizes $J_{1}$ and $J_{2}$ needs to be created and optimized. The optimization of this new function will give a solution from the Pareto front between $J_{1}^{*}$ and $J_{2}^{*}$. This is the easiest approach, but is highly dependent on the scalarizing function, which can be difficult to obtain, especially if $J_{1}$ and $J_{2}$ are of different nature.

If a goal programming approach is taken, then $I$ is taken as the ideal solution and a new optimization that minimizes the distance (measured according to a chosen criteria) from the solution to $I$ is solved to choose the best solution. This will result in an optimal solution from the Pareto front between $J_{1}^{*}$ and $J_{2}^{*}$. The final solution is dependent on the distance measurement chosen to evaluate the solution error, but this method allows a good evaluation of the loss in each objective function optimized.

In the classical lexicographic approach, if $J_{1}$ is prioritized, the multi-objective optimal solution is the point $J_{1}^{*}$, and, if $J_{2}$ is prioritized, it is $J_{2}^{*}$. Observing these solutions, it is noticeable the impact of the prioritizing order on the final solution. The lexicographic approach allows the prioritization of any function, but can cause significant losses in other variables when holding some at their optimal values.

When the augmented lexicographic multi-objective optimization is applied, the introduction of acceptable deviations allows the selection of the solutions $A$ and $B$ (depending on the prioritizing order), for which a small loss on one objective functions leads to a significant gain on the other. This proposed approach does not need the estimation of the complete Pareto set, allows the choice of any absolute or relative loss tolerances and also allows the adjusting of these tolerances at any step of the procedure, even after the final solution was found, allowing an interactive navigation through the Pareto front when needed. 


\subsubsection{Multi-Objective Optimization in Plantwide Control Design}

Plantwide control design is intrinsically a multi-objective problem with several conflicting objectives. However, even if a few design techniques include the optimization of a performance index, all of them consider only a single-objective formulation.

While some performance indicators can be easily merged into a composite function for single-objective formulation, others cannot be merged in an easy, intuitive, and efficient way.

For example, profit generated by final product and costs of energy and raw material can be easily integrated into a single composite cost function for the optimization of plant efficiency. In fact, most plantwide control design techniques with optimization included employ this cost function as objective function. On the other hand, the control effort of each actuator on the plant cannot be easily fully integrated into the main cost function, even if these efforts can influence the overall operating cost. While raw material and energy costs are a good indirect measure of control efforts, they are not a complete description of it.

For example, a controller that responds to disturbances with a fast and highly oscillating underdamped control action can reject the disturbance as well as a slow and non-oscillating overdamped control action and with almost the same cost. However, the excessive stress of actuators leads to their continuous deterioration and can insert new dynamics over time in the system due to actuator deterioration.

While actuator deterioration is hard to be measured directly and does not affect the process in a short time window, it can lead to severe loss of controllability and of control quality and profitability over time. Thus, while two different control systems can result in very similar operating costs at start of operation, one that stresses more the actuators will cause profitability decay over time and generate the need of earlier corrective maintenance.

Therefore, while the deterioration of process actuators cannot be easily integrated into a general cost function, due to the difficult of compatibility of engineering units of indicators and the high number of variables needed to describe it, the process 
behavior can be greatly affected over time and this future deterioration should be considered in the plantwide control design.

The introduction of constraints that limit the control efforts that lead to manipulator deterioration is a possible solution to this problem, but specifying the values for these constraints can be a difficult task and limit overall control optimality if not well performed. On the other hand, employing a specification such as "minimize control actions characteristics that lead to manipulator decay (such as oscillatory control efforts) without limiting optimal operation" in a multi-objective formulation, is a much more direct and flexible specification.

Concluding, while the employment of a single composite function can be a good approach in some design scenarios, the introduction of multi-objective formulation in plantwide control design can lead to significant gains in control quality and increased the flexibility of control system specifications.

\subsection{Optimal Control Tuning}

Typically, the tuning of the control system is not considered as part of the plantwide control design, falling as a posterior task that is part of the control implementation.

This section describes the control tuning problem, presents an optimal tuning technique applicable to any controller and control specifications and discusses the benefits of the inclusion of control tuning as part of the plantwide control design.

\subsubsection{The Optimal Control Tuning Problem}

The problem of optimal control tuning is stated as (Juliani, 2012):

"Given a plant and chosen a controller, a tuning for this controller is desired such that the behavior of the controlled system follows a set of requisites and is optimal according to a set of performance indexes."

An optimal tuner is depicted in Figure 3.6 (Juliani, 2012). 


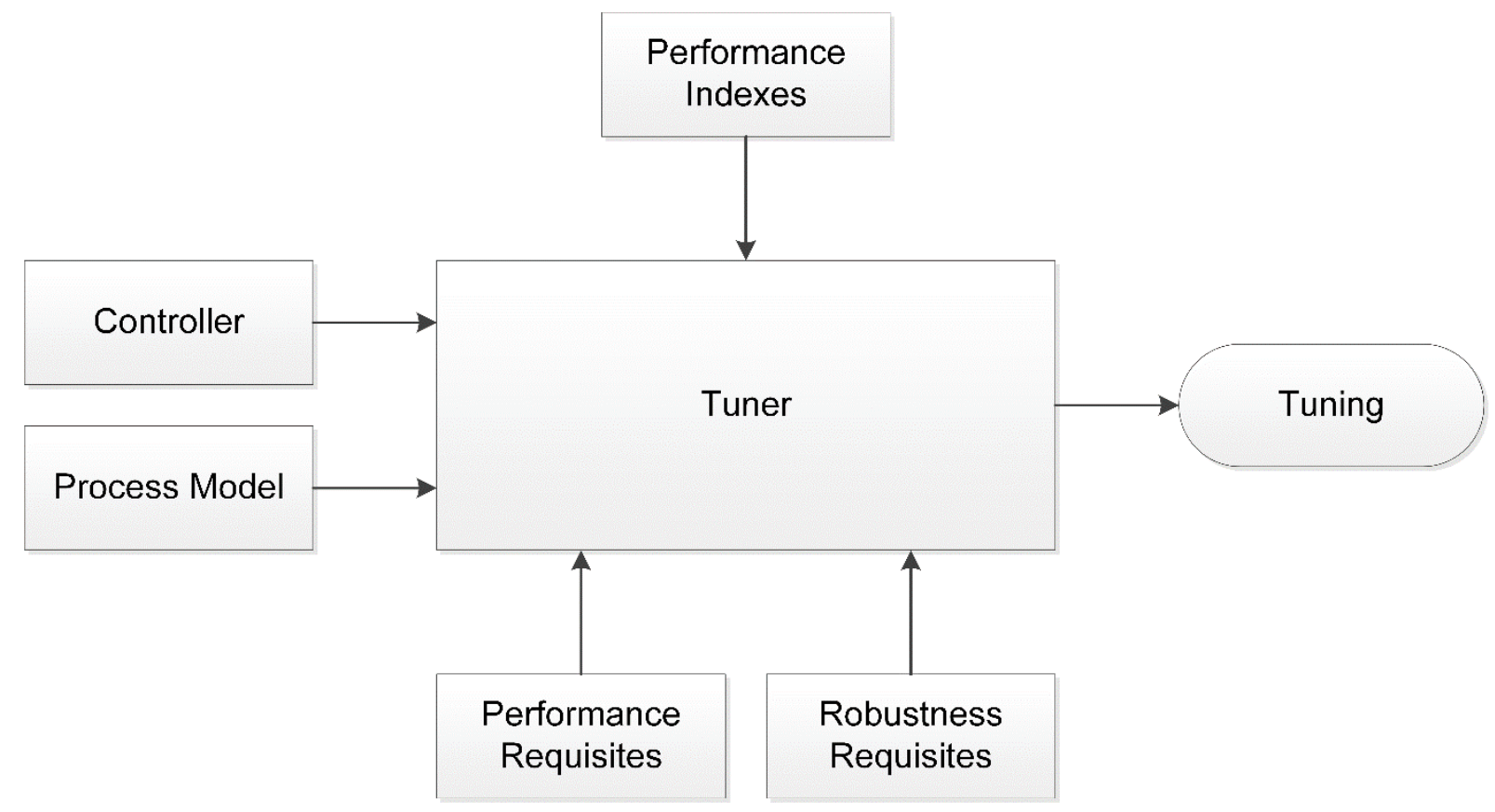

Figure 3.6 - Optimal Control Tuner (Juliani, 2012).

\subsubsection{A Procedure for Control Tuning}

To tune a controller, the following procedure is advised (Juliani, 2012):

1. Obtain a process model for the controlled, manipulated and relevant disturbance variables;

2. Choose the controller type to be employed;

3. Specify the performance requisites for the system;

4. Specify the robustness requisites for the system;

5. Specify the performance indexes to be optimized;

6. Write the problem in the standardized optimal tuning problem form;

7. Solve the resulting standard optimization problem and obtain the optimal tuning.

A comprehensive compendium of performance and robustness requisites and performance indexes is provided in (Juliani, 2012).

\subsubsection{Standard Optimal Tuning Problem Formulation}

If the control tuning problem is written in a standard optimization problem form, it can be solved by any standard optimization technique (Juliani, 2012), i.e., the control engineering problem is translated into an optimization problem that can be solved 
analytically (for some specific cases) or numerically by a series of widely available mathematical theories and computational tools.

A good standard representation for the tuning problem is (3.6) (Juliani, 2012).

subject to:

$$
S^{*}=\arg \min _{S} J=j\left(x_{p}(t), y_{p}(t), m_{v}(t), r(t), t\right)
$$

$$
\begin{aligned}
& \left\{\begin{array}{l}
\dot{x}_{p}(t)=a\left(x_{p}(t), u_{p}(t), t\right) \\
y_{p}(t)=h\left(x_{p}(t), u_{p}(t), t\right)
\end{array}\right\} \text { Process Model } \\
& \left\{\begin{array}{l}
\dot{x}_{c}(t)=g_{c}\left(x_{c}(t), c_{v}(t), d_{v}(t), r(t), S\right) \\
m_{v}(t)=h_{c}\left(x_{c}(t), r(t), S\right)
\end{array}\right\} \text { Controller Model }
\end{aligned}
$$

$\{S \in \mathcal{S}\}$ Controller Contructive Restrictions

$$
\begin{aligned}
& \left\{R_{P}\left(y_{p}(t), x_{p}(t), u_{p}(t)\right) \in \mathfrak{R}_{P}\right\} \text { Performance Requirements } \\
& \left\{R_{R}\left(y_{p}(t), x_{p}(t), u_{p}(t), \varepsilon_{p}\right) \in \mathfrak{R}_{R}\right\} \text { Robustness Requirements }
\end{aligned}
$$

in which $S^{*}$ is the optimal tuning parameter set, $S$ is the set of candidate tuning parameters, $J$ is a performance index (scalar), $j(\cdot)$ is a performance function, $x_{p}$ are the process states, $u_{p}$ are the process inputs (from which some or all are manipulated or disturbance variables, $y_{p}$ are the process outputs (from which some or all are controlled variables), $x_{c}$ are the controller states, $m_{v}$ are the manipulated variables, $c_{v}$ are the controlled variables, $d_{v}$ are the disturbance variables, $r$ are the references, $t$ is the time vector, $a(\cdot)$ represents the plant model, $h(\cdot)$ represents the plant output model, $g_{c}(\cdot)$ represents the controller model, $h_{c}(\cdot)$ represents the controller output model, $\mathcal{S}$ is the domain of the tuning parameters, $R_{P}$ are the performance requirements indicators, $R_{R}$ are the robustness requirements indicators, $\mathfrak{R}_{P}$ is the set of performance requirements, $\mathfrak{R}_{R}$ is the set of robustness requirements and $\varepsilon_{p}$ are the model errors.

The formulation (3.6) results in a standard optimization problem whose solution is a tuning parameter set $S^{*}$ from the tuning domain $\delta$ that optimizes a performance index (or a set of multi-objective indexes) $\mathrm{J}$, respecting the sets of performance and robustness requirements $\Re_{P}$ and $\Re_{R}$ for a controller $\left(g_{c}, h_{c}\right)$ acting on a plant $(a, h)$.

This formulation is general and can be applied different controller types, process model and control specifications. Moreover, it can be shown that any tuning technique is the solution of a particular standardized optimal tuning formulation. 


\subsubsection{Control Tuning in Plantwide Control Design}

Typically, "by the term plantwide control it is not meant the tuning and behavior of each control loop in a plant, but rather the control philosophy of the overall plant with emphasis on the structural decisions", which include the selection of manipulated and controlled variables, the arrangement of these variables and the choice of the controllers that will regulate the system (Larsson \& Skogestad, 2000).

Choosing between different controller types, or even between different control structures, however, is a tuning-dependent decision. The final controller behavior and, consequently, the plantwide performance, is not dependent only on the controller type, but is highly affected by its tuning (Juliani, 2012).

For example, it is very common in the literature to compare MPC and PID performances. While the MPC controller has a more complex structure and is capable of a better control, its implementations are heavily dependent on tuning (the process model employed and the controller adjustable parameters). On the other hand, PID is a simpler algorithm, with a simpler tuning process, and is reported to perform better in several applications. In fact, it cannot be stated that one of these controllers is a/ways the best option, but one or the other will be a better option depending on the implemented controllers, which depends on the controller tuning, which, in turn, depends on the process to be controlled, the control specifications, the employed tuning technique and the available data for the tuning (process model and expected control scenarios) (Juliani, 2012).

Therefore, there can be significant gains if control tuning or the tuning specification are taken into account in the plantwide control design, instead of choosing controllers only based on type and heuristics or assuming a perfect control.

\subsection{Concluding Remarks}

This chapter presented some theoretical tools that can be employed to enhance plantwide control design.

It is proposed that the classical approach of supposing a perfect model can be improved if the identification of a process model is included in the plantwide control 
design procedure, in order to best adapt the models to the design needs. When the concepts from System Identification are considered, this proposed approach also brings the advantage of limiting identification experiment costs (and length) while focusing on model precision where it is mostly needed.

Next, optimality in plantwide control is addressed, with the highlighting that, to be truly optimal, a control system must be optimal in a plantwide scale, considering all future consequences of its control actions and not only locally or for a short time window. This is not usually addressed in plantwide control design and should be included for a true optimal control to be achieved.

The concepts of Nash equilibrium and Pareto optimality are presented, and the importance of a plantwide control system seeking a Pareto optimality instead of a Nash equilibrium is discussed.

An analysis of multiple controllers acting on the same system shows that the cooperation between these controllers needs to be enforced by the plantwide control structure, once simply installing controllers to perform a local optimization does not lead to plantwide optimality.

Multi-objective optimization is also addressed and formally defined, with the proposal of an augmented lexicographic multi-objective optimization technique. The presented formulation can be applied to plantwide control design to allow the optimization of multiple conflicting performance indexes of different natures.

For the integration of control tuning into the plantwide control design, a general optimal control tuning technique is described and can be easily employed in the procedure for all the controllers designed.

The presented tools and results of the associated discussions will be employed in the next chapter to compose a new plantwide control design technique. 


\section{Proposed Techniques for Plantwide Control Design}

With the presented review taken into consideration and applying the described theoretical tools, a novel plantwide control design technique is here proposed. First, the technique is specified to include some novelties. Next, the plantwide control design procedure is described, followed by the proposal of an algorithm for integrated control structure selection and optimal controller tuning.

\subsection{Specification of a Novel Plantwide Control Design Technique}

The plantwide control design problem can be stated as:

Given a plant, a control system needs to be designed so that the controlled system follows a set of requisites and it is optimal according to a set of performance indexes.

While this problem statement is quite analogous to the proposed in (Juliani, 2012) for control tuning, it cannot be directly solved numerically as the latter. The main reason for this limitation is that, while the tuning problem is a search for an optimal tuning vector, the control design problem has an incredibly large number of decision variables, which results in a very large and complex combinatory problem, as previously stated. Moreover, available mathematical theories to solve such problem analytically are not applicable to large or complex cases and cannot be considered of practical usefulness.

Therefore, a procedure that solves the problem in a progressive and efficient way is desired. From a summary of the previous chapters, a plantwide control design technique with the following characteristics and contributions is sought:

Applicable to systems with any dimension;

Applicable to different types of controllers;

Support to true multi-objective control specifications;

Inclusion of system identification (and process modeling) as part of the procedure;

> Support to identification performable in various small experiments; 
$>\quad$ Inclusion of control tuning as part of the procedure;

$>\quad$ Final control system must satisfy Bellman's principle of optimality in a plantwide scale;

$>\quad$ Design of an optimal hierarchical control system;

$>\quad$ Each controller should be locally self-optimizing;

$>\quad$ The designed control must be robust optimal.

A novel plantwide control design technique that follows these specifications is proposed in the next section.

\subsection{Augmented Hierarchical Plantwide Control Design Technique}

The following augmented hierarchical plantwide control design technique is proposed:

I. Specification (top-down) steps:

1. Information gathering:

a) Gather information about the plant (operation data, existing models, models for similar plants, ...);

2. General control specifications:

a) Define control specifications (performance and robustness requirements);

b) Define performance objectives (one or more performance indicators), with respective loss tolerance and prioritizing order;

c) Identify candidate measurements $y$ and candidate manipulated variables $u$;

d) Identify steady-state degrees of freedom and determine the optimal steady-state operation conditions, including active constraints;

3. Plantwide control layer:

a) Identify plantwide objectives (typically a general cost function and operation constraints);

b) Obtain an approximate process model for the plantwide operational objectives; 
c) Determine measurable variables that directly affect the plantwide objective and tag them as candidate controlled variables;

d) Select economic optimization control type and structure for optimization of the main plantwide objectives subject to operation constraints;

4. Supervisory control layer:

a) Identify unit objectives (performance indicators for process units);

b) Obtain approximate dynamic models for the variables that need to be controlled for each unit, including variables with setpoints and constraints defined by the economic optimization layer and variables that affect unit objectives;

c) Identify measurable variables that directly affect the unit objectives and tag them as candidate controlled variables;

d) Select control type and structure for each unit for optimization of the unit objectives subject to the constraints imposed by the higher layer and operational constraints;

5. Regulatory control layer:

a) Identify local objectives (performance indicators for single variables or small groups of variables);

b) Obtain approximate dynamic models for the variables that need to be controlled in the regulatory layer, including variables with setpoints and constraints defined by the higher control layers and variables that affect local objectives;

c) Determine the measurable variables that affect the local objectives and tag them as candidate controlled variables;

d) Determine which process inputs affect each of the candidate controlled variables and tag them as candidate manipulated variables;

e) Select control type and structure for each variable or group of variables, optimizing local objectives subject to the constraints imposed by the higher layers and operational constraints;

II. Design (bottom-up) steps:

6. Regulatory control layer: 
a) Compile control specifications from the decisions taken in previous steps;

b) Obtain a precise dynamic model for the variables specified for the regulatory control layer;

c) Enforce cooperation between controllers through complementary specifications, constraints, or additional variables;

d) Complete control specifications with lacking information (such as robustness requirements);

e) Determine which candidate controlled and candidate manipulated variables will be used in the regulatory control layer and the parings between them;

f) Design and optimally tune the regulatory controllers, respecting the priority order for the objective functions;

g) Review the designed controllers, assess performance and ratify or rectify previous design decisions;

7. Supervisory control layer:

a) Compile control specifications from the decisions taken in previous steps;

b) Obtain a precise dynamic model for the variables specified for the supervisory control layer, including the designed regulatory controllers in closed loop;

c) Enforce cooperation between controllers through complementary specifications, constraints, or additional variables;

d) Include the setpoints for the regulatory layers in the list of candidate manipulated variables, substituting the manipulated variables used by such control layer;

e) Determine the controlled and manipulated variables for the supervisory layer and the pairings between them;

f) Complete control specifications with lacking information;

g) Design and optimally tune the supervisory controllers, respecting the priority order for the objective functions;

h) Review the designed controllers, assess performance and ratify or rectify previous design decisions;

8. Plantwide control layer: 
a) Compile control specifications from the decisions taken in previous steps;

b) Obtain a detailed model for the process, including the previously designed controllers;

c) Enforce cooperation between controllers through complementary specifications, constraints, or additional variables;

d) Complete control specifications with lacking information;

e) Assess the need of an independent plantwide control layer or if the plantwide optimization can be integrated to the controller designed to the supervisory control layer;

f) Include the setpoints for the supervisory control layer in the list of candidate manipulated variables, substituting the candidate variables that were committed to controllers in the lower layers;

g) Design and optimally tune the optimization controllers, respecting the priority order for the objective functions;

h) Review the designed controllers, assess performance and ratify or rectify previous design decisions;

9. General review and enhancements:

a) Review and evaluate the designed control system, making necessary adjustments.

The resulting control structure will enforce cooperation among controllers, Pareto optimality and robust optimality to the plant.

With the proposed technique, the following thesis is formulated:

For optimal operation in a productive system, higher layer controllers must optimize plantwide economic objectives, dynamically determining requirements for controllers subsidiary to them. Lower layer controllers must each optimize a local objective function while following restrictions imposed by controllers in higher layers and with higher priority.

This proposed technique was developed based on the presented discussion and techniques previously designed by other authors, following mainly the Skogestad's 7-step Plantwide Control Procedure (Larsson \& Skogestad, 2000), (Skogestad, 2000a), (Skogestad, 2000b), (Skogestad, 2004a). 


\subsection{Optimal Multi-Objective Technique for Integrated Control Structure Selection and Controller Tuning}

Two of the most important tasks of the plantwide control design are the selection of $\mathrm{I} / \mathrm{O}$ pairings and controller tuning, performed on the design steps for all control layers. This section proposes a technique to perform both tasks in an integrated and simultaneous way.

This technique aims at obtaining a control that is optimal according to a multiobjective specification through the optimization of the variable pairings and the control tuning. Another main characteristic is that the control tuning is performed in a multivariable approach to take into consideration the coupling effects, as initially proposed in the optimal tuning technique described in Section 3.7.

The proposed technique has the advantages of being applicable to any process model and of providing a rigorous formulation, without the need of heuristics. Furthermore, the $\mathrm{I} / \mathrm{O}$ pairing constraints can be easily changed to accept other types of pairings, such as a multi-variable control pairing.

The main limitation of this approach is that the problem formulation and resolution can be too complex for large or complex systems, being more adequate for application to small and simple mid-scale processes. For larger or more complex cases, this must be used to solve sub-problems determined by the more general approach proposed in the previous section.

The proposed formulation (4.1) is based on the multi-objective multi-variable optimal control tuning technique proposed in (Juliani, 2012), (Juliani \& Garcia, 2012) and (Juliani \& Garcia, 2014) and the concepts of an optimization approach to plantwide control presented in (Jørgensen \& Jørgensen, 2000).

In (4.1), $S$ is the set of control tuning parameters, $P$ is the matrix of variable pairings, $j(\cdot)$ are the objective functions to be optimized, $x(t), y(t)$ and $u(t)$ are, respectively, the states, outputs and inputs for the plant (sub index $p$ ) and controller (sub index $c$ ), $d(t)$ are the process disturbances, $r(t)$ is the desired reference and $t$ is the time vector. 


$$
\{S, P\}^{*}=\arg \min _{\mathrm{S}, \mathrm{P}} J=j_{l}\left(x_{p}(t), y_{p}(t), u_{p}(t), r(t), t\right)
$$

subject to:

$$
\begin{aligned}
& \left\{\begin{array}{l}
j_{k}(\cdot)=\alpha_{k} \cdot j_{k}^{*}+\delta_{k} \\
\alpha_{k} \cdot j_{k}^{*}+\delta_{k} \geq j_{k}^{*} \\
k=1, \ldots, l-1
\end{array}\right\} \text { Multiobjective Specification } \\
& \left\{\begin{array}{l}
\dot{x}_{p}(t)=a\left(x_{p}(t), u_{p}(t), d_{x}(t), t\right) \\
y_{p}(t)=h\left(x_{p}(t), u_{p}(t), d_{y}(t), t\right)
\end{array}\right\} \text { Process Model } \\
& \left\{\begin{array}{l}
\dot{x}_{c}(t)=g_{c}\left(x_{c}(t), c_{v}(t), d_{v}(t), r(t), S\right) \\
m_{v}(t)=h_{c}\left(x_{c}(t), r(t), S\right)
\end{array}\right\} \text { Controller Model } \\
& \{S \in \mathcal{S}\} \text { Controller Constructive Restrictions } \\
& \left\{R_{P}\left(y_{p}(t), x_{p}(t), u_{p}(t)\right) \in \Re_{P}\right\} \text { Performance and Operation Constraints } \\
& \left\{R_{R}\left(y_{p}(t), x_{p}(t), u_{p}(t), \varepsilon_{p}\right) \in \mathfrak{R}_{R}\right\} \text { Robustness Constraints } \\
& \left\{\begin{array}{l}
u_{p}=P \cdot y_{c}(t) \\
\sum_{k=1}^{N_{u_{p}}} p_{i k} \leq 1 \\
\sum_{k=1}^{N_{C}} p_{k j} \leq 1 \\
p_{i j} \in\{0,1) \\
i=1: N_{y_{p}} \\
j=1: N_{u_{p}}
\end{array}\right\} \text { Input/Output Pairing }
\end{aligned}
$$

The first set of optimization constraints describes a lexicographic approach to the multi-objective problem. In this approach, the more important objectives are optimized first, and then the less important ones are optimized with the priority objectives kept close to their optimal values according to the relative and absolute tolerances $\alpha$ and $\delta$.

For the process and controller models, a nonlinear state-space representation is considered. Simplifications of this representation can be employed, and even simulation programs for which there is no explicit mathematical model.

Controller constructive restrictions $\mathcal{S}$ describe constraints to the tuning parameters, such as minimum and maximum values and precision. The set of constraints $\mathfrak{R}_{P}$ contains performance and operation constraints specified to the process. 
The $1 / O$ pairing constraint determines that, for the paring matrix $P$, each controller can be assigned to only one plant output and one plant input, and that not all plant inputs and outputs need to be assigned to a controller. This last consideration allows the formulation to be applicable to non-square systems. This constraint should be adjusted according to the plant structure demanded by the employed controller.

This technique can be applied with the plantwide control design procedure proposed in the previous section to determine the $1 / O$ pairing and control tuning for the candidate controlled and measured variables in each design step. Application examples and a complete complex design to illustrate the application of both techniques will be presented in Chapters 5 and 6 .

This technique provides a control that is optimal according to a multi-objective specification through the optimization of the variable pairings and the controller tuning, which is performed in a multi-variable approach in order to take into consideration the coupling effects. It can be applied to any process model, without the need of heuristics. Furthermore, the $\mathrm{I} / \mathrm{O}$ pairing constraints can be easily changed to accept other types of pairings, such as a multi-variable control pairing. ${ }^{2}$

In summary, I/O pairings and controller tuning are simultaneously optimized to satisfy a multi-objective specification.

\subsection{Concluding Remarks}

In this chapter, a new technique for plantwide control design and another for integrated $\mathrm{I} / \mathrm{O}$ pairing and control tuning were proposed.

The main innovations of the proposed Augmented Hierarchical Plantwide Control Design Technique are:

Integration of system identification in the plantwide design procedure, which allows the identification experiments to be better planned, improving the identification results and reducing experiment costs;

\footnotetext{
${ }^{2}$ This proposed technique was published in (Juliani \& Garcia, 2017a).
} 
> Integration of optimal control tuning in the plantwide design procedure, which allows better decisions to be made, taking in consideration the expected behavior of the controllers instead of supposing ideal control. This also results in the final implemented control system behaving being closer to the designed system, reducing the need of adjustments and redesigns;

$>\quad$ Support to multi-objective specifications, which allows any number of objective functions (independent or conflicting objectives) to be specified and optimized in a multi-objective manner.

The main limitation of the proposed technique is that it needs a real or simulated plant to be applied, as does the other existing mathematical and hybrid plantwide control design techniques. So, the proposed technique can be applied after a process has been defined and either built or implemented in a simulator. This approach also admits that the problem of plant operability was properly addressed in the process design and that it can successfully work in at least one operating point.

To deal with the problems of $\mathrm{I} / \mathrm{O}$ pairing and optimal control tuning, the Optimal Multi-Objective Technique for Integrated Control Structure Selection and Controller Tuning was proposed. This technique solves both problems simultaneously, and also allows multi-objective specifications and the use of any process and controller types.

Both proposed techniques will be applied to industrial benchmarks in the next two chapters to validate and illustrate their use. 


\section{Investigatory Tests and Application Examples}

In this chapter, the proposals of this thesis are applied to simulated systems and the results evaluated.

First, five classical industrial benchmarks are considered, and all possible control structures are exhaustively implemented and tested. These experiments seek to evaluate the relation between control variables pairings, tuning and performance.

The second set of experiments applies the proposed techniques to develop a plantwide control system to two larger classic industrial benchmarks, demonstrating its applicability.

\subsection{Investigation of Control Structure Selection and Controller Tuning for PID Controllers Applied to Classical Industrial Benchmarks}

An investigation into PID controllers input/output (I/O) pairing is performed. From the classic I/O pairing techniques encountered in literature, it is expected that only a few configurations will result in acceptable control and that the I/O pairing can be defined independently of the final control tuning, however, it is verified that the controller tuning influences the final performance as much as the $\mathrm{I} / \mathrm{O}$ pairing, and that a bad pairing can be compensated by a good control tuning to achieve a satisfactory control. This investigation demonstrates that an optimal tuning should be sought simultaneously with the best I/O pairing, and not after the pairing has been determined.

This section presents an investigation into I/O pairing for PID controllers and its relationship with the controllers tuning and system performance. The following objectives are established for this investigation:

$>\quad$ Verify the feasibility of different PID I/O pairings;

$>\quad$ Verify the relation between PID I/O pairing and tuning;

$>\quad$ Assess the benefits of including optimal control tuning in the control structure selection procedure.

Classical control structure selection techniques include the RGA (Bristol, 1966) and NI (Niderlinski, 1971) approaches, with many variants of these indexes proposed 
by other authors. Other approaches can also be found, such as optimization-based techniques (Robinson, et al., 2001) and branch and bound methods (Cao \& Prabirkumar, 2005), (Yi \& Vinay, 2008), (Kariwala \& Cao, 2009), (Kariwala \& Yi, 2010).

Such classical techniques suggest that, for a given system, only one or a few I/O variable pairings will result in an acceptable control. The first objective of this investigation is to study and quantify this effect in some simulated examples.

Secondly, the relation between I/O pairing and control tuning is investigated. It is shown that the inclusion of the control tuning in the control structure selection problem can greatly improve the resulting control system quality.

It is also sought to show how the optimal tuning procedure can be coupled with the $\mathrm{I} / \mathrm{O}$ pairing decisions and how the final results can be improved with this approach.

For this investigation, five industrial benchmarks were chosen: a $2 \times 2$ distillation column (Wood \& Berry, 1973), a 2x2 ill-conditioned high purity distillation column (Skogestad, et al., 1988), a binary column (Luyben, 1989), a 3x3 industrial furnace (Zanin, 1993) and a 3x3 distillation column (Ogunnaike, et al., 1983).

PID controllers are optimally tuned for all possible $1 / O$ pairings for all plants, according to the procedure presented in Section 3.7. All controllers are tuned minimizing the settling time and the rise time, keeping the overshoot and undershoot constrained, in this order, prioritizing the controlled variables from $y_{1}$ to $y_{n}$.

All the PID controllers are implemented in the digital form with sampling time of 1 second, unless differently stated. All process inputs are limited according to operation ranges of the plants. When available, a reference tuning is also presented.

In summary, the five chosen plants allow 14 different I/O pairings, which are all evaluated. 21 control systems with 53 PID controllers are tuned and evaluated. For all plants, steps are applied to each setpoint and disturbance. The simulation results are presented and analyzed next.

\subsubsection{Tests with the Wood and Berry Distillation Column Benchmark}

The first benchmark presented is the distillation column (Wood \& Berry, 1973) shown in Figure 5.1. 


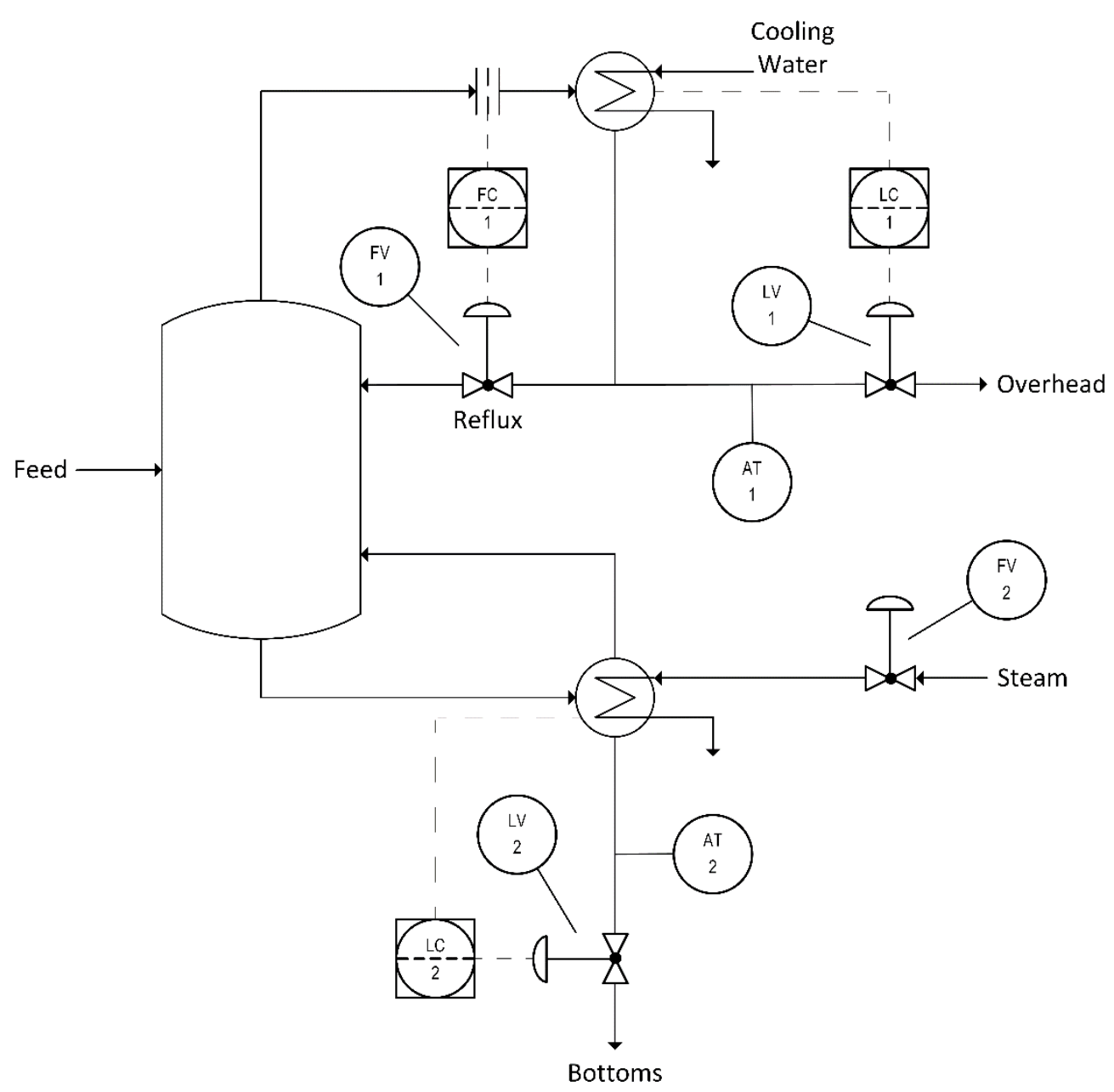

Figure 5.1 - Wood and Berry distillation column (Wood \& Berry, 1973).

This model is linear with time delays and has two controlled variables: top and bottom compositions; two manipulated variables: reflux and steam flows and one disturbance variable: the feed flow. The nominal values for the variables are $96 \%$ and $0.5 \%$ for the top and bottom compositions, respectively, and $0.0325,0.285$ and 0.0408 $\mathrm{lb} / \mathrm{s}$ for the reflux, steam and feed flows. The corresponding process model is shown in Equations (5.1), (5.2) and (5.3), which describe the effects of the manipulated and the disturbance variables, respectively, on the plant outputs.

$$
\begin{aligned}
& y(s)=G(s) \cdot u(s)+H(s) \cdot d(s) \\
& G(s)=\left[\begin{array}{ll}
\frac{768}{1002 \cdot s+1} \cdot e^{-60 \cdot s} & \frac{396}{645 \cdot s+1} \cdot e^{-420 \cdot s} \\
\frac{-1.134}{1260 \cdot s+1} \cdot e^{-180 \cdot s} & \frac{-1.164}{864 \cdot s+1} \cdot e^{-180 \cdot s}
\end{array}\right] \\
& H(s)=\left[\begin{array}{l}
\frac{228}{894 \cdot s+1} \cdot e^{-480 \cdot s} \\
\frac{294}{792 \cdot s+1} \cdot e^{-180 \cdot s}
\end{array}\right]
\end{aligned}
$$


Both possible pairings are tested and PID controllers with PI structure in the parallel form are optimally tuned for each configuration, according to the general specifications previously defined. The resulting optimal tuning parameters are shown in Table 5.1, in engineering units.

Table 5.1 - Optimal PID Tuning Parameters for the Wood and Berry Benchmark

\begin{tabular}{c|c|c}
\hline Controller Parameter & Y1xU1, Y2xU2 & Y1xU2, Y2xU1 \\
\hline$K_{P_{u_{1}}}$ & 0.0057 & -0.0002 \\
\hline$K_{I_{u_{1}}}$ & $1.3 \cdot 10^{-5}$ & $-1.7 \cdot 10^{-6}$ \\
\hline$K_{P_{u_{2}}}$ & -0.0009 & -0.005 \\
\hline$K_{I_{u_{2}}}$ & $-4 \cdot 10^{-6}$ & $-1.1 \cdot 10^{-5}$ \\
\hline
\end{tabular}

To evaluate the controllers, steps are applied to each setpoint and to the feed flow at the instants 500, 6000 and 12000, respectively. Process responses are shown in Figures 5.2 and 5.3.

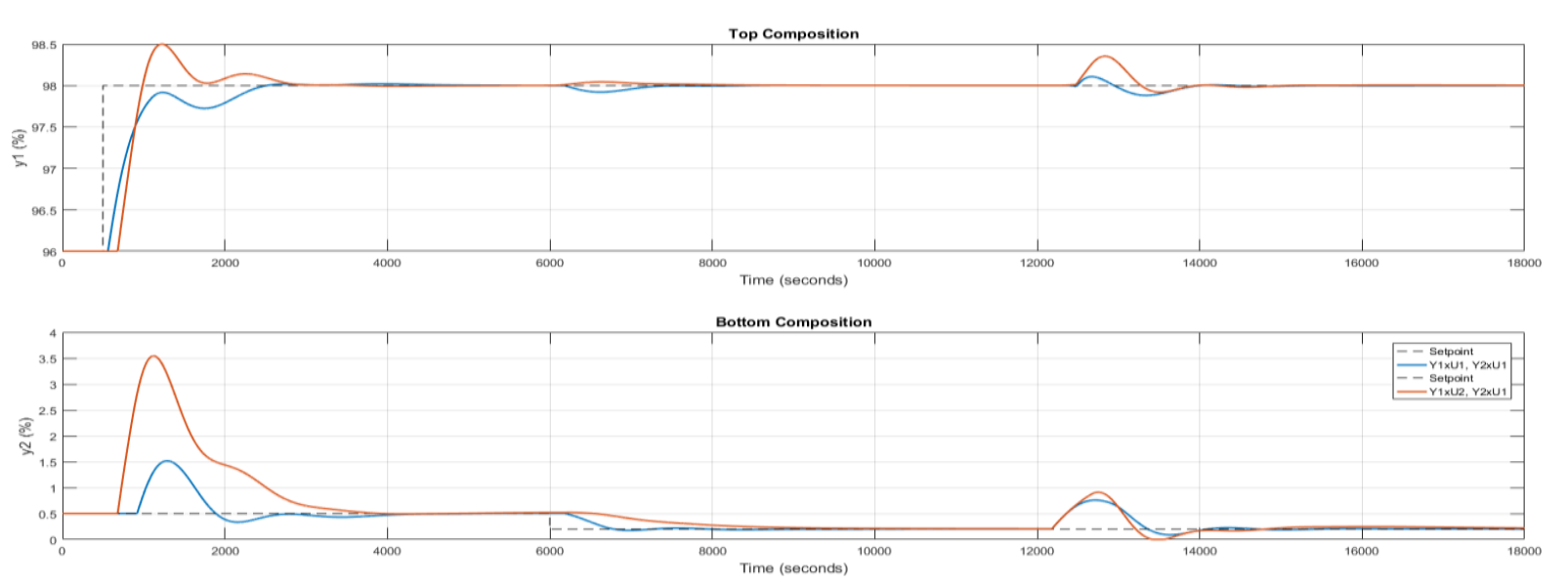

Figure 5.2 - Results for the Wood and Berry Distillation Column - Process Outputs.
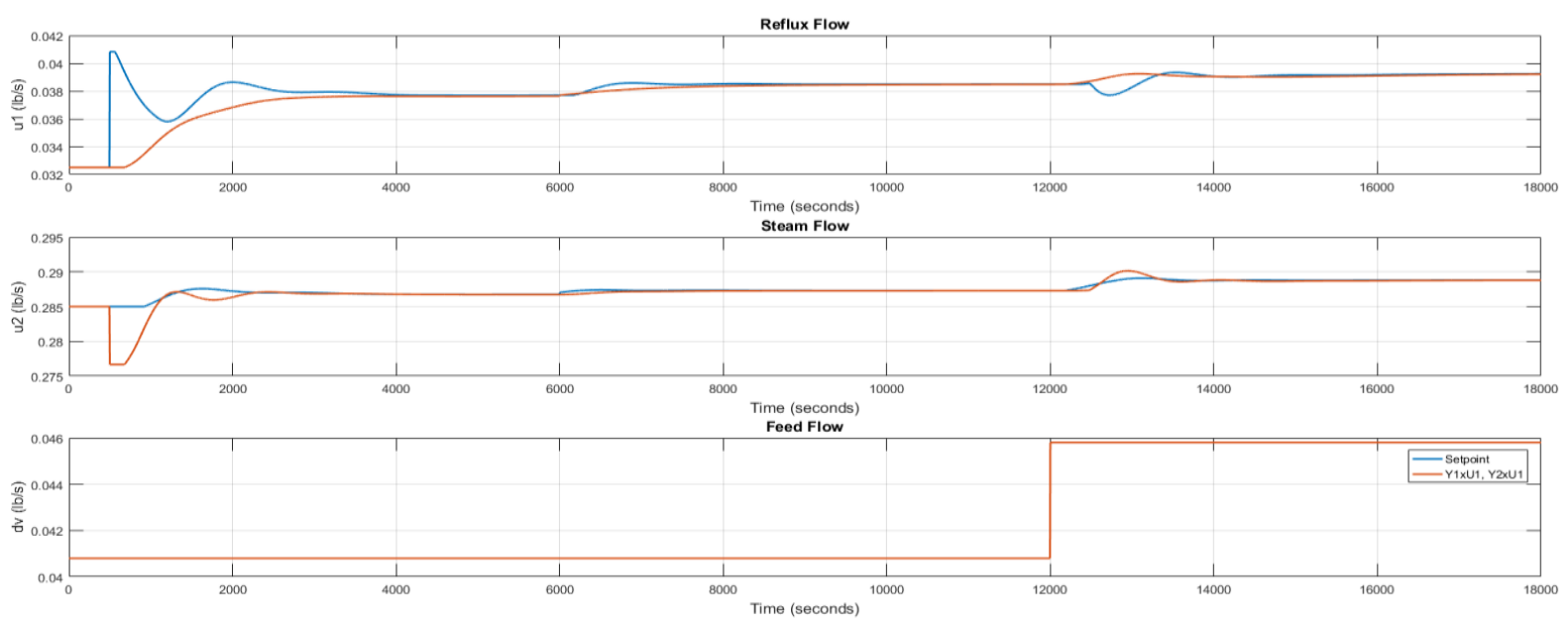

Figure 5.3 - Results for the Wood and Berry Distillation Column - Process Inputs. 
It is possible to note that both configurations result in acceptable controls, but with different dynamic characteristics. The corresponding performance indicators are shown in Table 5.2, with the best indexes highlighted.

Table 5.2 - Performance Indicators for the Wood and Berry Benchmark

\begin{tabular}{|c|c|c|c|}
\hline Process & \multirow[t]{2}{*}{ Performance Indicator } & \multirow[t]{2}{*}{ Y1xU1, Y2xU2 } & \multirow[t]{2}{*}{$\overline{Y 1 x U 2, Y 2 x U 1}$} \\
\hline Variable & & & \\
\hline \multirow{7}{*}{ 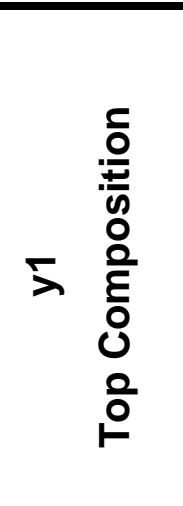 } & Settling Time (s) & 1860 & 2200 \\
\hline & Rise Time (s) & 464 & 240 \\
\hline & Overshoot (\%) & 0.91 & 25 \\
\hline & Returning Time (s) & 986 & 0 \\
\hline & Maximum Deviation (\%) & 0.08 & 0.04 \\
\hline & Regulatory Returning Time (s) & 1740 & 1220 \\
\hline & Regulatory Maximum Deviation (\%) & 0.12 & 0.35 \\
\hline \multirow{7}{*}{ 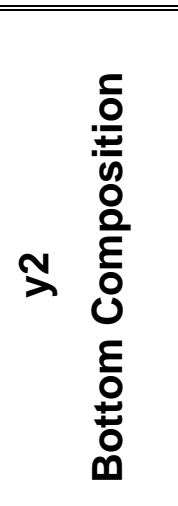 } & Settling Time (s) & 2848 & 3977 \\
\hline & Rise Time (s) & 441 & 1987 \\
\hline & Overshoot (\%) & 0.09 & 0 \\
\hline & Returning Time (s) & 3157 & 2945 \\
\hline & Maximum Deviation & 1.02 & 3.2 \\
\hline & Regulatory Returning Time (s) & 1920 & 1920 \\
\hline & Regulatory Maximum Deviation (\%) & 0.56 & 0.71 \\
\hline
\end{tabular}

In Table 5.2, the returning time corresponds to how long a variable takes to return to its setpoint after a change in the setpoint of the other process variables, and the regulatory returning time corresponds to how long the variable takes to return to its setpoint after a disturbance occurs.

From the analysis of Figures 5.2 and 5.3 and of Table 5.2, it can be concluded that both possible $\mathrm{I} / \mathrm{O}$ pairings can be employed to control this system, if the PID controllers are properly tuned. Therefore, there is not a correct $\mathrm{I} / \mathrm{O}$ pairing for this process, but the choice of pairing to be used depends on the analysis of the expected dynamic behavior of the system.

From the results, the $[\mathrm{Y} 1 \mathrm{xU} 1, \mathrm{Y} 2 \mathrm{xU} 2]$ configuration is better for this system, due to faster response times and lower overshoot and deviations from the setpoints. 


\subsubsection{Tests with the III-Conditioned High Purity Distillation Column Benchmark}

The second benchmark is an ill-conditioned high purity distillation column (Skogestad, et al., 1988), which is used as a linear model with two controlled and two manipulated variables. The plant is shown in Figure 5.4, with its corresponding linear model presented converted to seconds presented in Equations (5.4), (5.5) and (5.6).

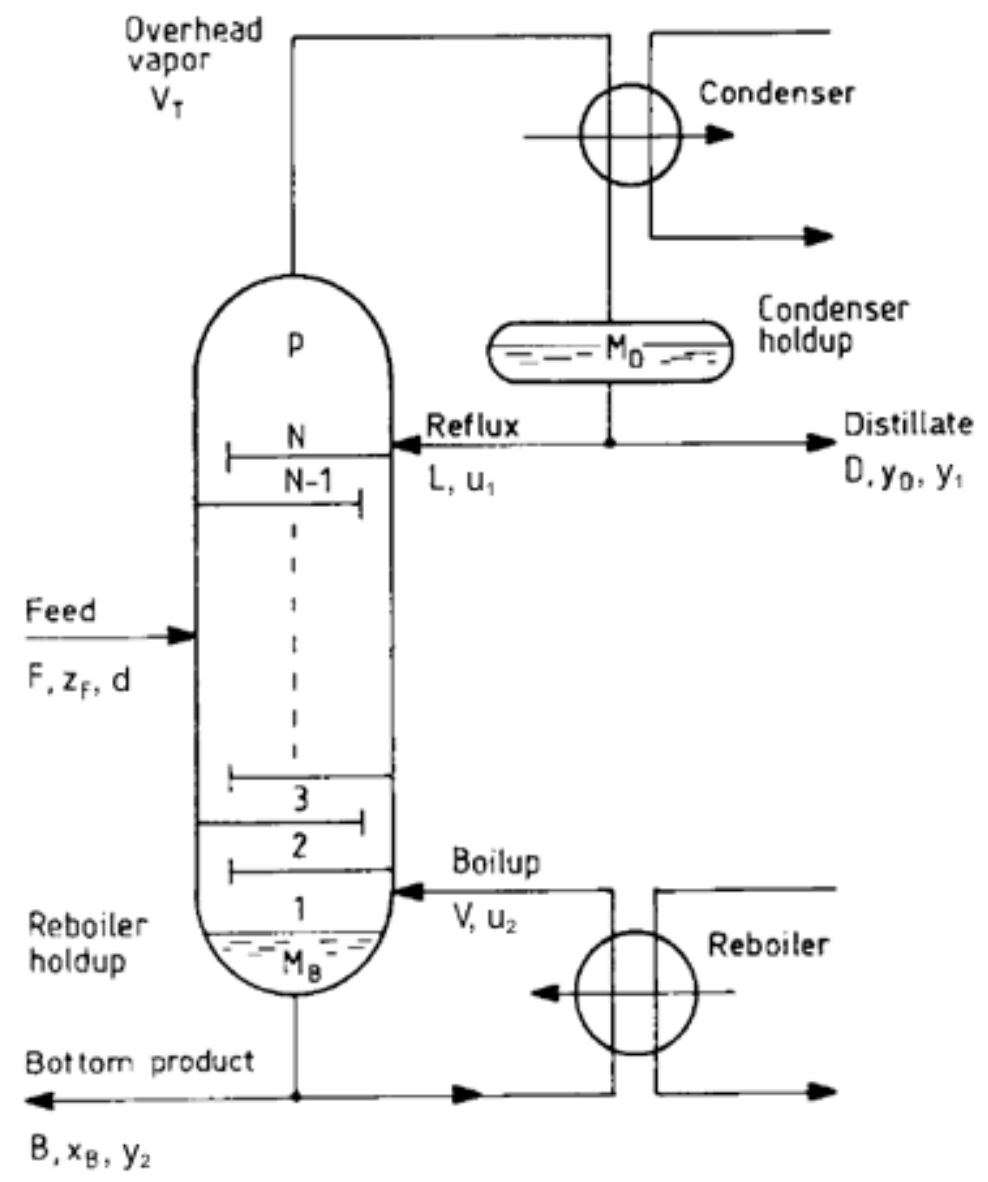

Figure 5.4 - III-Conditioned High Purity Distillation Column (Skogestad, et al., 1988).

$$
\begin{aligned}
& y(s)=G(s) \cdot u(s)+H(s) \cdot d(s) \\
& G(s)=\left[\begin{array}{cc}
\frac{5268}{11640 \cdot s+1} & \frac{-37640 \cdot s-51.84}{1.048 \cdot 10^{7} \cdot s^{2}+12540 \cdot s+1} \\
\frac{6492}{11640 \cdot s+1} & \frac{-6.826 \cdot 10^{6} \cdot s-6576}{1.048 \cdot 10^{7} \cdot s^{2}+12540 \cdot s+1}
\end{array}\right] \\
& H(s)=\left[\begin{array}{c}
\frac{1}{6000 \cdot s+1} \\
\frac{1}{6000 \cdot s+1}
\end{array}\right]
\end{aligned}
$$


The plant outputs are the top $\left(y_{D}\right)$ and bottom $\left(x_{B}\right)$ compositions, the inputs are the reflux $(L)$ and boilup $(V)$ and the disturbance variable is the feed flow $(F)$.

Both possible $\mathrm{I} / \mathrm{O}$ pairings are implemented and PID controllers are optimally tuned for each configuration, following the specifications previously defined. The resulting optimal tuning parameters, in engineering units, are shown in Table 5.3, which includes also the original tuning. All the PID controllers have a sampling time of 1 minute.

Table 5.3 - Optimal PID Tuning Parameters for the Wood and Berry Benchmark

\begin{tabular}{c|c|c|c}
\hline Controller Parameter & Original Tuning & Y1xU1, Y2xU2 & Y1xU2, Y2xU1 \\
\hline$K_{P_{u_{1}}}$ & 0.0038 & 0.03 & 0.03 \\
\hline$K_{I_{u_{1}}}$ & $3.3 \cdot 10^{-7}$ & $5 \cdot 10^{-5}$ & $3 \cdot 10^{-5}$ \\
\hline$K_{P_{u_{2}}}$ & -0.0030 & -0.02 & 0.01 \\
\hline$K_{I_{u_{2}}}$ & $-2.7 \cdot 10^{-7}$ & $-3 \cdot 10^{-5}$ & $3 \cdot 10^{-5}$ \\
\hline
\end{tabular}

To evaluate the controls, steps are applied to each setpoint, at instants 500 and 6000 , respectively, and to the disturbance variable at instant 10000 . Incremental system responses are shown in Figures 5.5 and 5.6.

Both possible configurations are stable and satisfy control requirements. The final choice depends on which impacts more on the overall process performance. For a better analysis and comparison, performance indicators are shown in Table 5.4.
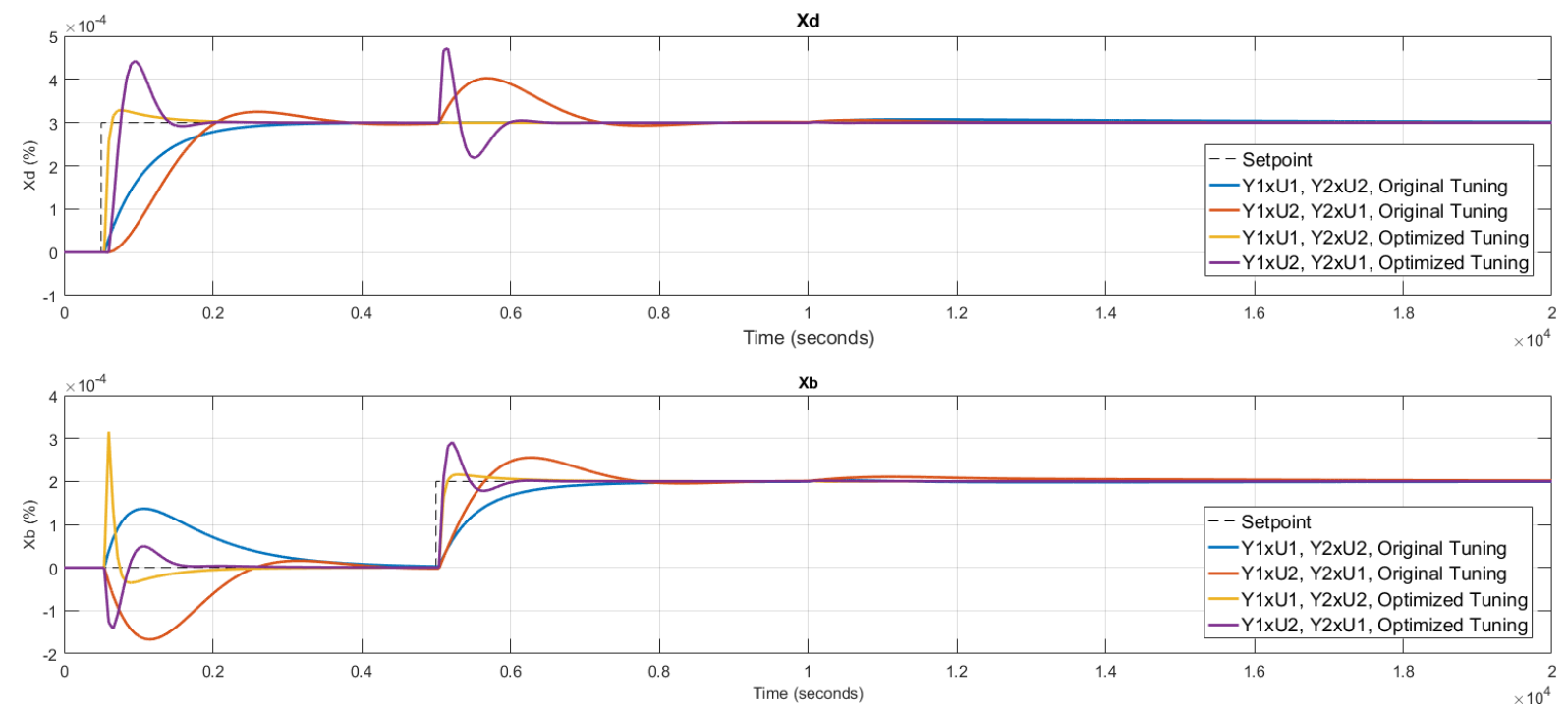

Figure 5.5 - Results for the High Purity Distillation Column - Process Outputs. 


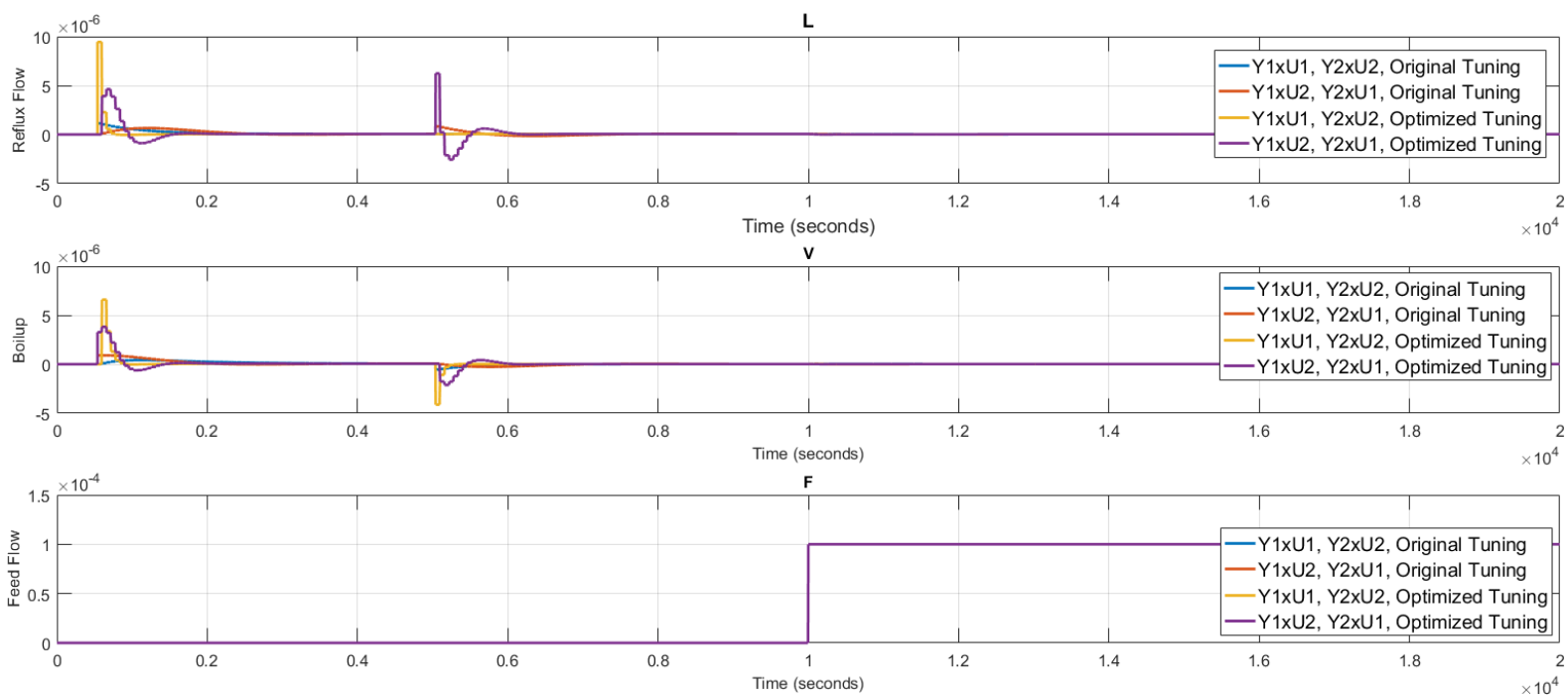

Figure 5.6 - Results for the High Purity Distillation Column - Process Inputs.

Table 5.4 - Performance Indicators for the High Purity Distillation Column Benchmark

\begin{tabular}{|c|c|c|c|c|}
\hline $\begin{array}{l}\text { Process } \\
\text { Variable }\end{array}$ & Performance Indicator & $\begin{array}{l}\text { Original } \\
\text { Tuning }\end{array}$ & $\begin{array}{l}\text { Y1xU1, } \\
\text { Y2xU2 }\end{array}$ & $\begin{array}{l}\text { Y1xU2, } \\
\text { Y2xU1 }\end{array}$ \\
\hline \multirow{7}{*}{ 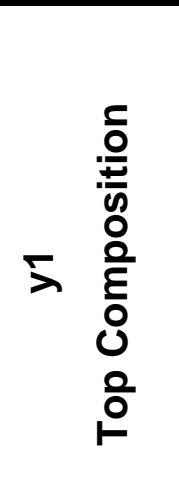 } & Settling Time (s) & 2186 & 1100 & 842 \\
\hline & Rise Time (s) & 2186 & 221 & 105 \\
\hline & Overshoot (\%) & 0 & 9.6 & 47.6 \\
\hline & Returning Time (s) & 0 & 0 & 918 \\
\hline & Maximum Deviation & $1.7 \cdot 10^{-4}$ & 0 & 0 \\
\hline & Regulatory Returning Time (s) & 3460 & 0 & 0 \\
\hline & Regulatory Maximum Deviation & $0.08 \cdot 10^{-4}$ & 0 & 0 \\
\hline \multirow{7}{*}{ 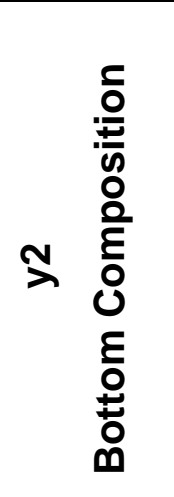 } & Settling Time (s) & 2747 & 1262 & 968 \\
\hline & Rise Time (s) & 2747 & 155 & 98 \\
\hline & Overshoot (\%) & 0 & 8 & 45.7 \\
\hline & Returning Time (s) & 4085 & 1666 & 1081 \\
\hline & Maximum Deviation & $1.37 \cdot 10^{-4}$ & $1.43 \cdot 10^{-4}$ & $3.15 \cdot 10^{-4}$ \\
\hline & Regulatory Returning Time (s) & 0 & 0 & 0 \\
\hline & Regulatory Maximum Deviation & 0 & 0 & 0 \\
\hline
\end{tabular}

From the analysis of Figures 5.5 and 5.6 and Table 5.4, it can be concluded that, as in the previous case, both possible I/O pairings can be employed to control this system, if the PID controllers are properly tuned. 
From the observed results, the $[\mathrm{Y} 1 \mathrm{xU} 1, \mathrm{Y} 2 \mathrm{xU} 2]$ configuration presents the lower deviations from the setpoints, while the [Y1xU2, $Y 2 x U 1]$ configuration presents faster responses, with lower rise, settling and returning times, at the cost of higher overshoots and deviations. The final choice depends on which is more important for the control: speed or smoothness.

\subsubsection{Tests with the Binary Distillation Column Benchmark}

The third plant tested is a binary distillation column with a nonlinear rigorous model containing two controlled and two manipulated variables. The plant is shown in Figure 5.7. The complete nonlinear process model can be found in (Luyben, 1989).

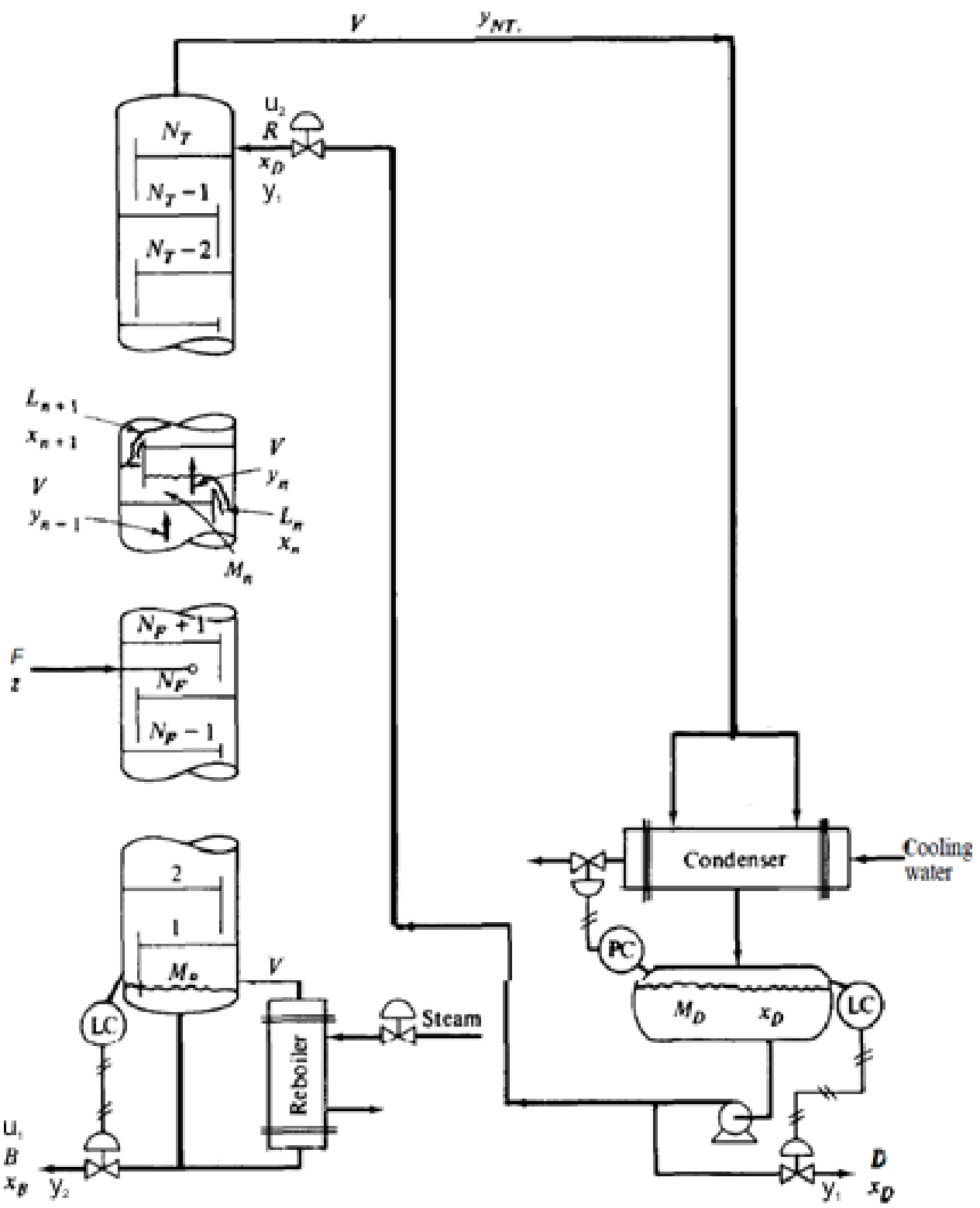

Figure 5.7 - Binary Distillation Column (Luyben, 1989). 
The process controlled variables are the top distillate $\left(x_{D}\right)$ and bottom $\left(x_{B}\right)$ compositions and the manipulated variables are bottom product flow rate $(B)$ and the reflux flow rate $(R)$.

PID controllers are designed for both possible I/O pairings. The final tuning parameters are shown in Table 5.5 in engineering units. For this process, optimal tunings are obtained for sampling times of 1 second and 1 minute.

Table 5.5 - Optimal PID Tuning Parameters for the Binary Distillation Column Benchmark

\begin{tabular}{c|c|c|c:c}
\hline $\begin{array}{c}\text { Controller } \\
\text { Parameter }\end{array}$ & $\begin{array}{c}\text { Y1xU1, Y2xU2 } \\
\text { min }\end{array}$ & $\begin{array}{c}\text { Y1xU2, Y2xU1 } \\
\text { min }\end{array}$ & $\begin{array}{c}\text { Y1xU1, Y2xU2 } \\
\text { sec }\end{array}$ & $\begin{array}{c}\text { Y1xU2, Y2xU1 } \\
\text { sec }\end{array}$ \\
\hline$K_{P_{u_{1}}}$ & -5 & 0.1 & -50 & 15 \\
\hline$K_{I_{u_{1}}}$ & -0.1 & 0.04 & -5 & 1.6 \\
\hline$K_{D_{u_{1}}}$ & -10 & 0 & -10 & 5 \\
\hline$K_{P_{u_{2}}}$ & 1 & 2 & 20 & 50 \\
\hline$K_{I_{u_{2}}}$ & 0.1 & 0.2 & 5 & 5 \\
\hline$K_{D_{u_{2}}}$ & 10 & 0 & 30 & 50 \\
\hline$T_{s}(s)$ & 60 & 60 & 1 & 1 \\
\hline
\end{tabular}

Steps are then applied to each setpoint to evaluate control performance. Results for the PID controllers operating at sampling time of 1 minute are shown in Figures 5.8 and 5.9, with steps applied to the setpoints at instants 100 and 2500. Next, the results for the controllers with sampling time of 1 second are shown in Figures 5.10 and 5.11 .
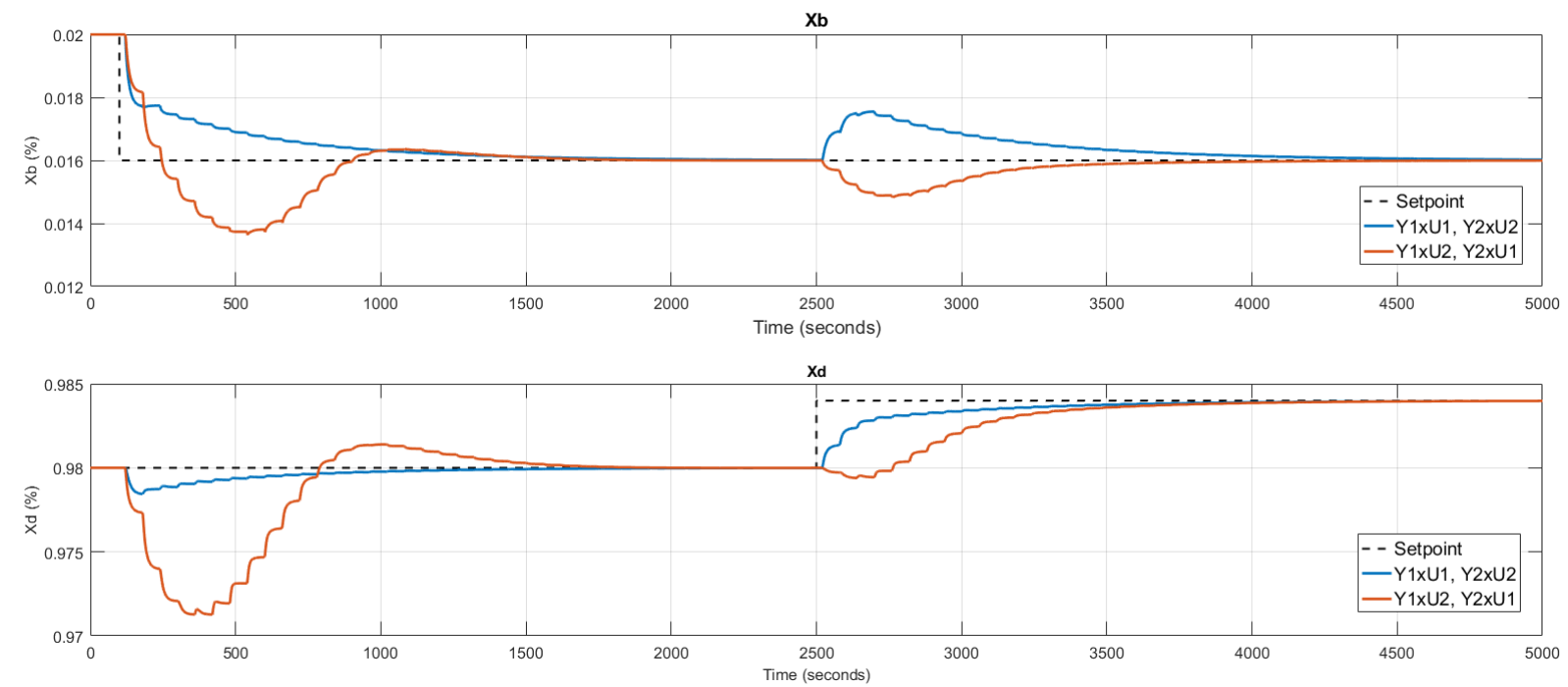

Figure 5.8 - Results for the Binary Column for PID controllers with $\mathrm{T}=60 \mathrm{~s}-$ Process Outputs. 

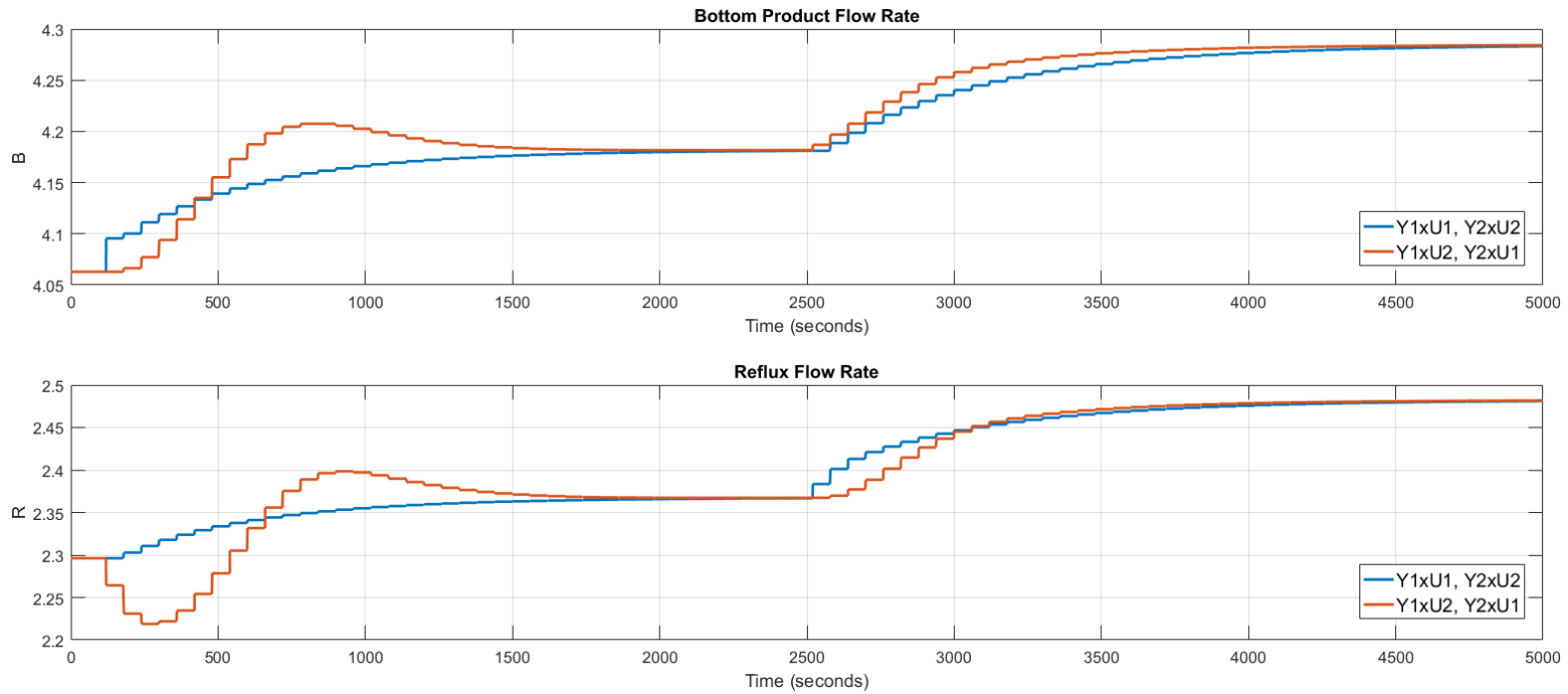

Figure 5.9 - Results for the Binary Column for PID controllers with $T=60 \mathrm{~s}-$ Process Inputs.
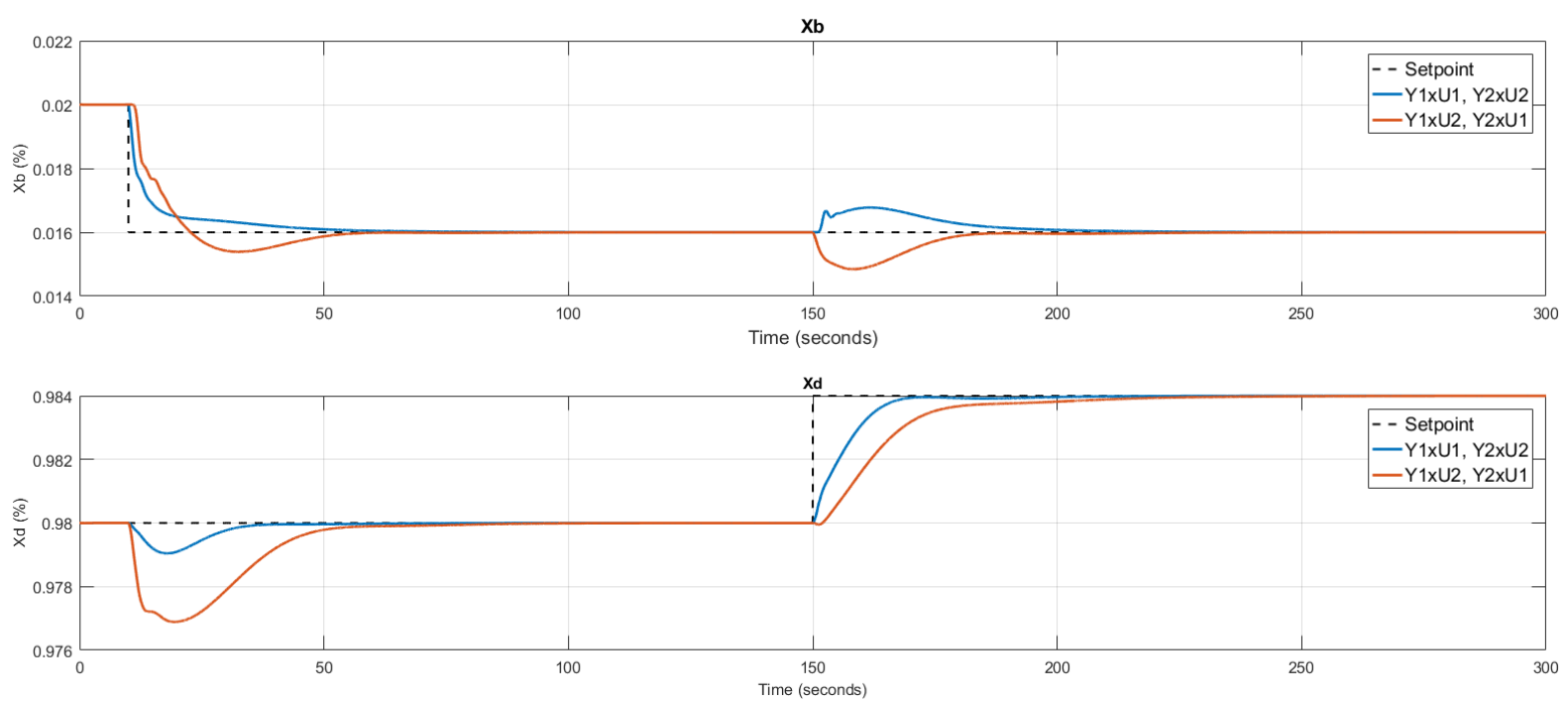

Figure 5.10 - Results for the Binary Column for PID controllers with T = $1 \mathrm{~s}-$ Process Outputs.
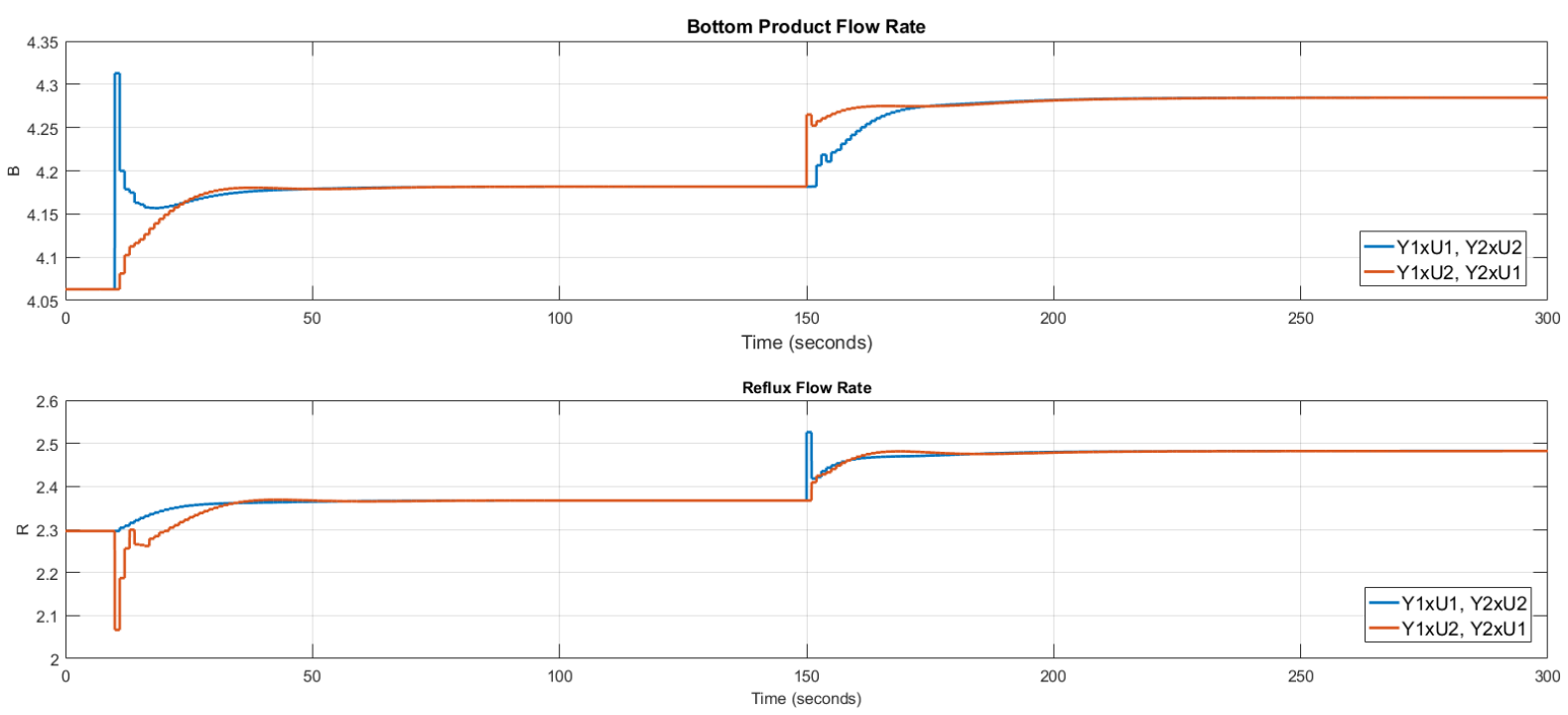

Figure 5.11 - Results for the Binary Column for PID controllers with T = $1 \mathrm{~s}$ - Process Inputs. 
Both I/O pairings are stable and satisfy the requirements, but it observed that the control operating with a sampling time of 1 second is much faster. The final choice of best pairing depends on which impacts more on the overall process performance. For a better analysis and comparison, performance indicators are shown in Table 5.6, in which the best performance indexes are highlighted.

Table 5.6 - Performance Indicators for the Binary Distillation Column Benchmark

\begin{tabular}{|c|c|c|c|c|c|}
\hline $\begin{array}{l}\text { Process } \\
\text { Variable }\end{array}$ & $\begin{array}{c}\text { Performance } \\
\text { Indicator }\end{array}$ & $\begin{array}{c}\text { Y1xU1, } \\
\text { Y2xU2 } \\
\text { min }\end{array}$ & $\begin{array}{c}\text { Y1xU2, } \\
\text { Y2xU1 } \\
\text { min }\end{array}$ & $\begin{array}{c}\text { Y1xU1, } \\
\text { Y2xU2 } \\
\text { sec }\end{array}$ & $\begin{array}{c}\text { Y1xU2, } \\
\text { Y2xU1 } \\
\text { sec }\end{array}$ \\
\hline \multirow{5}{*}{ 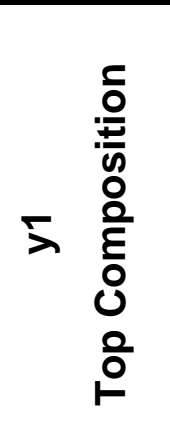 } & Settling Time (s) & 1630 & 1792 & 49 & 45 \\
\hline & Rise Time (s) & 149 & 140 & 16 & 18 \\
\hline & Overshoot (\%) & 0 & 10.63 & 0 & 2.05 \\
\hline & Returning Time (s) & 1046 & 621 & 24 & 22 \\
\hline & $\begin{array}{l}\text { Maximum } \\
\text { Deviation }\end{array}$ & 0.313 & 0.16 & 0.085 & 0.14 \\
\hline \multirow{5}{*}{ 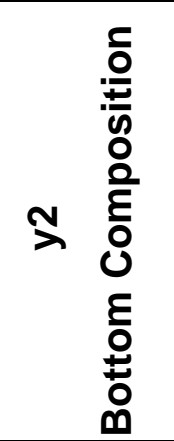 } & Settling Time (s) & 995 & 700 & 16 & 55 \\
\hline & Rise Time (s) & 207 & 654 & 12.5 & 21 \\
\hline & Overshoot (\%) & 5 & 0 & 0 & 0 \\
\hline & Returning Time (s) & 700 & 1736 & 29 & 39 \\
\hline & $\begin{array}{l}\text { Maximum } \\
\text { Deviation }\end{array}$ & 0.36 & 1.34 & 0.12 & 0.41 \\
\hline
\end{tabular}

Figures 5.7, 5.8, 5.10 and 5.11 and Table 5.6 show that, as in the previous cases, both possible $\mathrm{I} / \mathrm{O}$ pairings can be employed to control this system if the PID controllers are properly tuned.

From the observed results, the [Y1xU1, Y2xU2] presents better performance for both tested sampling times, due to their lower deviations from the setpoints, and the controllers with sampling time of 1 second present much better responses than those with sampling time of 1 minute.

\subsubsection{Tests with the Industrial Furnace Benchmark}

The forth evaluated benchmark is an industrial furnace (Zanin, 1993), described by the linear model shown in Equations (5.7), (5.8) and (5.9) with sampling time of 1 
minute, with three controlled, three manipulated and one disturbance variables. The three controlled variables are the vessel level $\left(y_{1}\right)$, convection output temperature $\left(y_{2}\right)$ and radiation output temperature $\left(y_{3}\right)$, the three manipulated variables are the flow to radiation $\left(u_{1}\right)$, flow to convection $\left(u_{2}\right)$ and fuel pressure $\left(u_{3}\right)$ and the disturbance variable is the feed flow $(d)$.

$$
\begin{aligned}
& y(z)=G(z) \cdot u(z)+H(z) \cdot d(z) \\
& G(z)=\left[\begin{array}{ccc}
\frac{0.1736 \cdot 10^{-4}}{1-z^{-1}} & \frac{0.1736 \cdot 10^{-4}}{1-z^{-1}} & 0 \\
0 & \frac{-0.4150 \cdot 10^{-4}}{1-0.9992 \cdot z^{-1}} & \frac{0.0083}{1-0.9992 \cdot z^{-1}} \\
\frac{-3.2+9.7 z^{-1}-9.3 z^{-2}+3 z^{-3}}{1-4 z^{-1}+5.9 z^{-2}-3.9 z^{-3}+z^{-4}} \cdot 10^{-6} & 0 & \frac{-2.4+7.0 z^{-1}-6.9 z^{-2}+2.24 z^{-3}}{1-4 z^{-1}+5.9 z^{-2}-3.9 z^{-3}+z^{-4}} \cdot 10^{-3}
\end{array}\right] \\
& H(z)=\left[\begin{array}{c}
0.1736 \cdot 10^{-4} \\
\frac{1-z^{-1}}{0} \\
0
\end{array}\right]
\end{aligned}
$$

This model has some zeroes in the transfer matrix, which gives two possible $\mathrm{I} / \mathrm{O}$ pairings. PID controllers with sampling time of 1 minute are designed for each one. The resulting parameters are shown, in engineering units, in Table 5.7, which also shows the original tuning.

Table 5.7 - Optimal PID Tuning Parameters for the Industrial Furnace Benchmark

\begin{tabular}{c|c|c|c}
\hline $\begin{array}{c}\text { Controller } \\
\text { Parameter }\end{array}$ & $\begin{array}{c}\text { Y1xU1, Y2xU2, } \\
\text { Y3xU3 Original }\end{array}$ & $\begin{array}{c}\text { Y1xU1, Y2xU2, } \\
\text { Y3xU3 Optimal }\end{array}$ & $\begin{array}{c}\text { Y1xU2, Y2xU3, } \\
\text { Y3xU1 Optimal }\end{array}$ \\
\hline$K_{P_{u_{1}}}$ & -75 & -300 & -10 \\
\hline$K_{I_{u_{1}}}$ & -0.375 & -3 & -0.0075 \\
\hline$K_{D_{u_{1}}}$ & 0 & -1 & -1 \\
\hline$K_{P_{u_{2}}}$ & -2 & -100 & -300 \\
\hline$K_{I_{u_{2}}}$ & -0.05 & -1 & -3 \\
\hline$K_{D_{u_{2}}}$ & 0 & -0.1 & -1 \\
\hline$K_{P_{u_{3}}}$ & 0.15 & 0.1 & 10 \\
\hline$K_{I_{u_{3}}}$ & $1.5 \cdot 10^{-6}$ & $5 \cdot 10^{-5}$ & 0.1 \\
\hline$K_{D_{u_{3}}}$ & 0 & 0.1 & 0.1 \\
\hline
\end{tabular}

To evaluate control performance, steps are applied to each setpoint at instants 500,15000 and 30000, respectively, and to the disturbance variable at instant 45000 . Results are shown in Figures 5.12 and 5.13. 

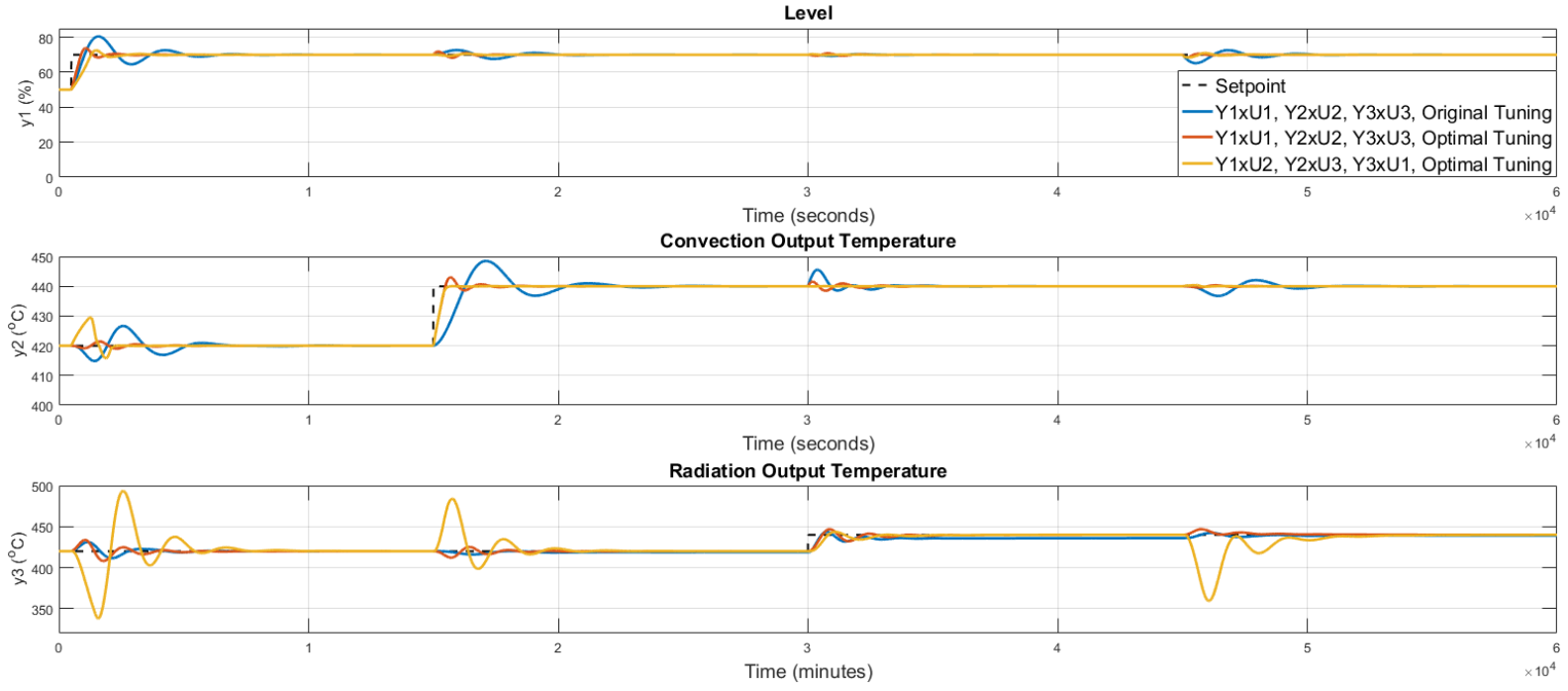

Figure 5.12 - Results for the Industrial Furnace - Process Outputs.

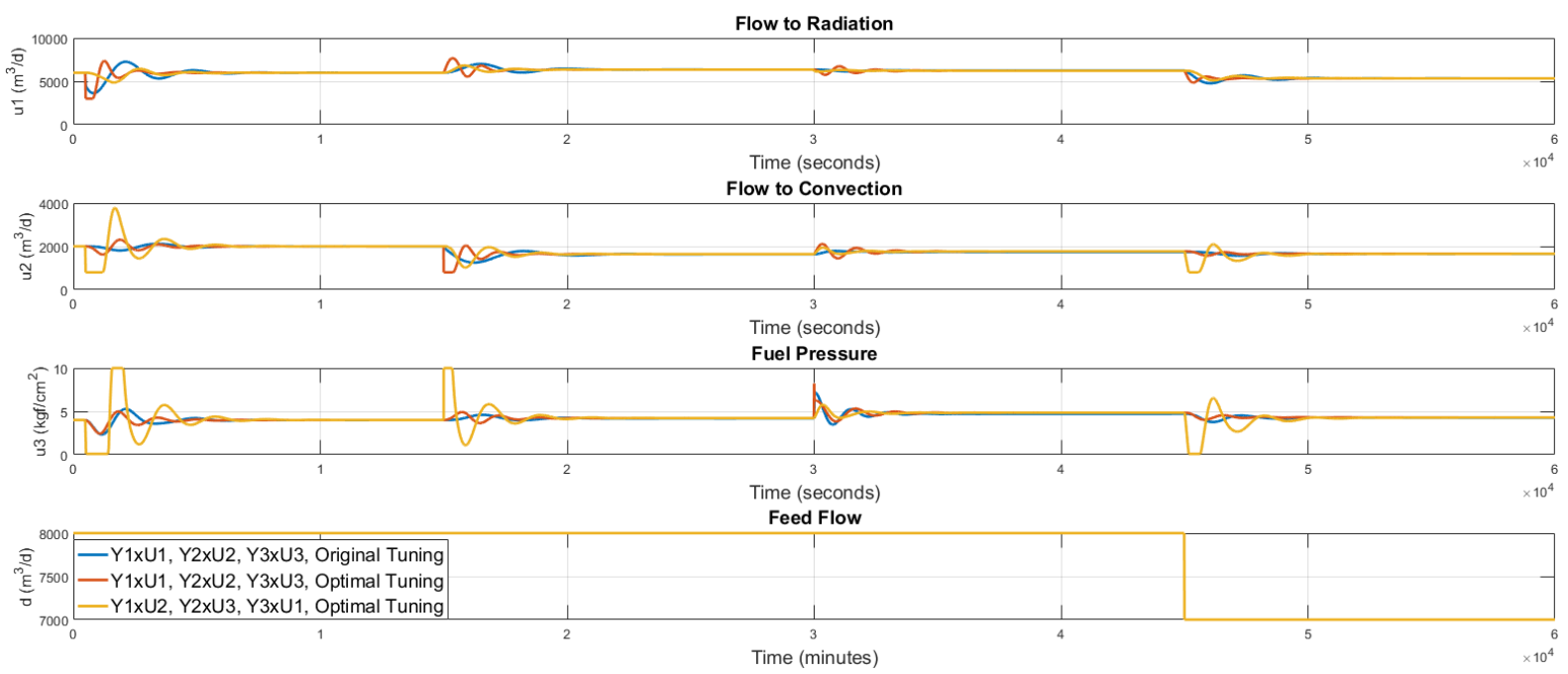

Figure 5.13 - Results for the Industrial Furnace - Process Inputs.

As can be seen, the original tuning is not capable of following the setpoint in $Y 3$ during the complete test, while all optimal tunings can remove the steady-state error. All possible configurations with optimized tunings are stable and satisfactory, but present slightly different dynamic responses.

Table 5.8 shows the performance indicators for each configuration, highlighting the best ones. In Table 5.8, the $1^{\text {st }}$ returning time indicates the time needed for a variable to return to steady-state when a setpoint change is applied to another variable, the $2^{\text {nd }}$ returning time is the same indicator calculated for a second setpoint change on another variable. 
Table 5.8 - Performance Indicators for the Industrial Furnace Benchmark

\begin{tabular}{|c|c|c|c|c|}
\hline $\begin{array}{l}\text { Process } \\
\text { Variable }\end{array}$ & Performance Indicator & $\begin{array}{l}\text { Y1xU1, } \\
\text { Y2xU2, } \\
\text { Y3xU3 } \\
\text { Original }\end{array}$ & $\begin{array}{l}\text { Y1xU1, } \\
\text { Y2xU2, } \\
\text { Y3xU3 } \\
\text { Optimal }\end{array}$ & $\begin{array}{l}\text { Y1xU2, } \\
\text { Y2xU3, } \\
\text { Y3xU1 } \\
\text { Optimal }\end{array}$ \\
\hline \multirow{7}{*}{ 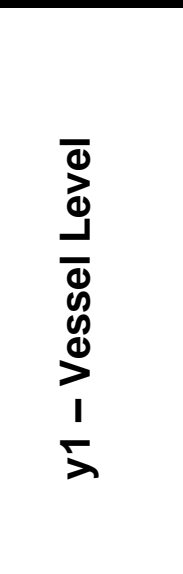 } & Settling Time (s) & 5699 & 1932 & 2068 \\
\hline & Rise Time (s) & 488 & 354 & 734 \\
\hline & Overshoot (\%) & 52.6 & 19.1 & 12.7 \\
\hline & $1^{\text {st }}$ Returning Time (s) & 4750 & 2000 & 1550 \\
\hline & $2^{\text {nd }}$ Returning Time (s) & 1280 & 1570 & 0 \\
\hline & $\begin{array}{l}\text { Regulatory Returning Time } \\
\text { (s) }\end{array}$ & 4940 & 810 & 1150 \\
\hline & Maximum Deviation & 4.8 & 1.82 & 1.52 \\
\hline \multirow{7}{*}{ 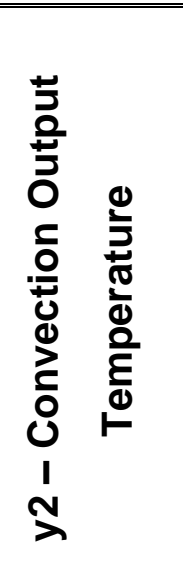 } & Settling Time (s) & 6860 & 2120 & 530 \\
\hline & Rise Time (s) & 1170 & 420 & 420 \\
\hline & Overshoot (\%) & 42.5 & 15.5 & 0 \\
\hline & $1^{\text {st }}$ Returning Time (s) & 5884 & 2709 & 1673 \\
\hline & $2^{\text {nd }}$ Returning Time (s) & 2960 & 2240 & 0 \\
\hline & $\begin{array}{l}\text { Regulatory Returning Time } \\
\text { (s) }\end{array}$ & 5290 & 0 & 0 \\
\hline & Maximum Deviation & 6.7 & 1.5 & 9.6 \\
\hline \multirow{7}{*}{ 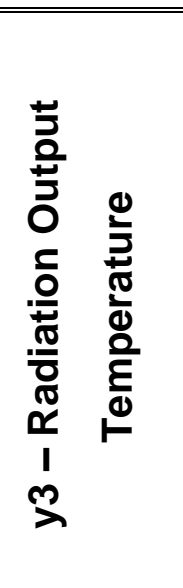 } & Settling Time (s) & $\infty$ & 5350 & 4900 \\
\hline & Rise Time (s) & 490 & 530 & 750 \\
\hline & Overshoot (\%) & 18.5 & 34.0 & 18.5 \\
\hline & $1^{\text {st }}$ Returning Time (s) & 4977 & 4897 & 9012 \\
\hline & $2^{\text {nd }}$ Returning Time (s) & $\infty$ & 4150 & 7410 \\
\hline & $\begin{array}{l}\text { Regulatory Returning Time } \\
\text { (s) }\end{array}$ & $\infty$ & 8760 & 9900 \\
\hline & Maximum Deviation & 11.2 & 13.8 & 73.5 \\
\hline
\end{tabular}

Figures 5.12 and 5.13 and Table 5.8 show that, as in the previous cases, all possible I/O pairings can be employed to control this system, if the PID controllers are properly tuned. The [Y1xU2, Y2xU3, Y3xU1] configuration produces better results for $Y 2$, and results very close to the optimal for $Y 1$, as well as the best step response for 
$\mathrm{Y} 3$, however, it presents the largest deviations for $\mathrm{Y} 3$. The [Y1xU1, Y2xU2, Y3xU3] configuration with optimal tunings presents lower deviations from the setpoints, at the cost of higher overshoots and slower responses. The choice between these two options depends on which variable most affects the overall plant performance.

\subsubsection{Tests with the $3 \times 3$ Distillation Column Benchmark}

The next benchmark investigated is the $3 \times 3$ distillation binary distillation column (Ogunnaike, et al., 1983), with three controlled, three manipulated and two disturbance variables, shown in Figure 5.14.

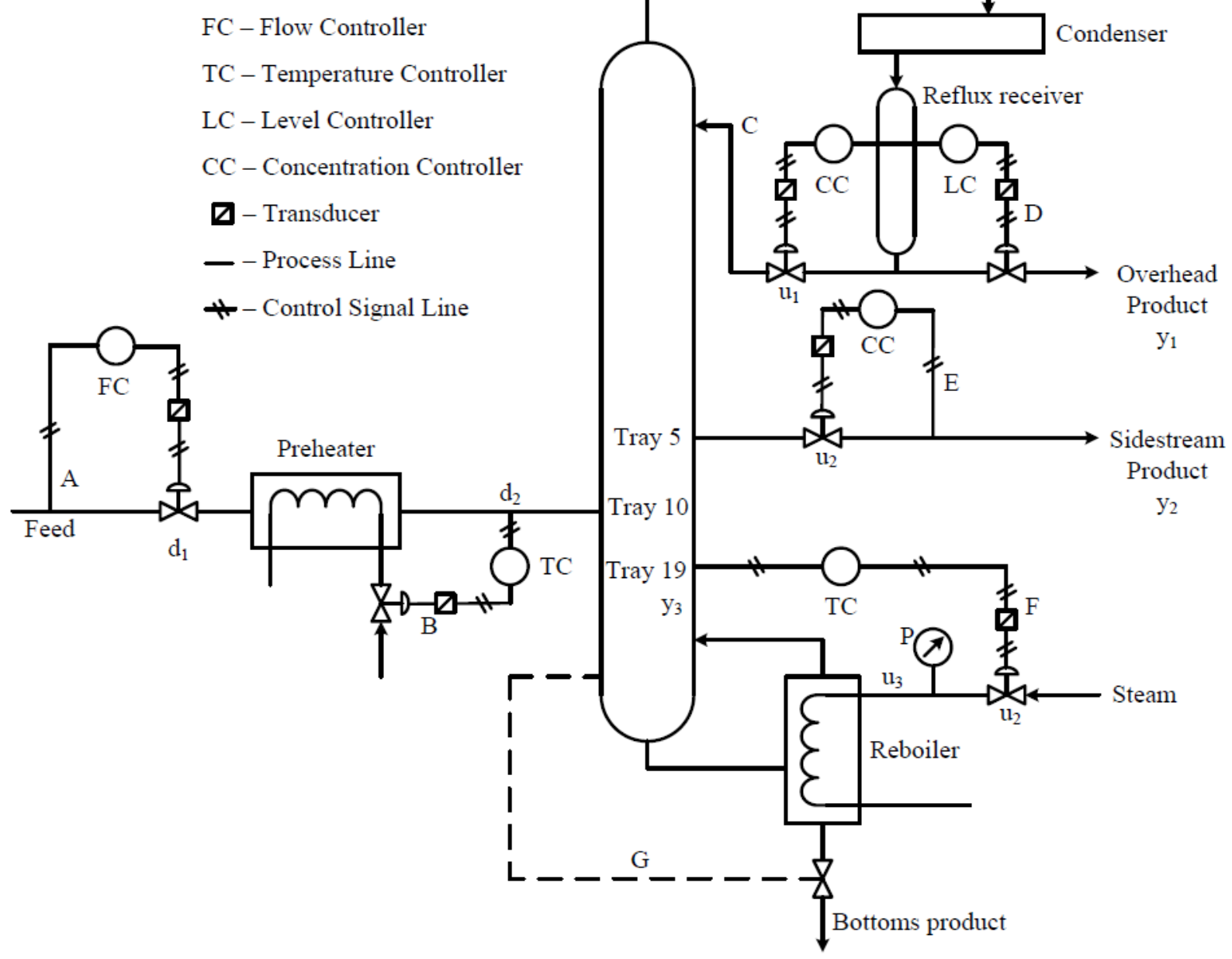

Figure $5.14-3 \times 3$ binary distillation column (Ogunnaike, et al., 1983).

For the ethanol-water system modeled, the controlled variables are the overhead ethanol mole fraction $\left(y_{1}\right)$, the side stream ethanol mole fraction $\left(y_{2}\right)$ and the tray 19 temperature $\left(y_{3}\right)$, corresponding to the bottom composition. The manipulated variables are the reflux flow rate $\left(u_{1}\right)$, the side stream product flow rate $\left(u_{2}\right)$ and the 
reboiler stream pressure $\left(u_{3}\right)$. The unmeasured disturbances are the feed flow rate $\left(d_{1}\right)$ and the feed temperature $\left(d_{2}\right)$. This process is modeled by a linear and full transfer function matrix with time delays, shown in Equations (5.10), (5.11) and (5.12), in minutes.

$$
\begin{aligned}
& y(s)=G(s) \cdot u(s)+H(s) \cdot d(s) \\
& G(s)=\left[\begin{array}{ccc}
\frac{0.66 e^{-2.6 s}}{6.7 s+1} & \frac{-0.61 e^{-3.5 s}}{8.64 s+1} & \frac{-0.0049 e^{-s}}{9.06 s+1} \\
\frac{1.11 e^{-6.5 s}}{3.25 s+1} & \frac{-2.36 e^{-3 s}}{5.0 s+1} & \frac{-0.012 e^{-1.2 s}}{7.09 s+1} \\
\frac{-34.68 e^{-9.2 s}}{8.15 s+1} & \frac{46.2 e^{-9.4 s}}{10.9 s+1} & \frac{0.87 \cdot(11.61 s+1) e^{-s}}{(3.89 s+1) \cdot(18.8 s+1)}
\end{array}\right] \\
& H(s)=\left[\begin{array}{cc}
\frac{0.14 e^{-12 s}}{6.2 s+1} & \frac{-(26.32 s+1) \cdot 0.0011 e^{-2.66}}{(7.85 s+1) \cdot(14.63 s+1)} \\
\frac{0.53 e^{-10.5 s}}{6.9 s+1} & \frac{-(19.62 s+1) \cdot 0.0032 e^{-3.44 s}}{(7.29 s+1)(8.94 s+1)} \\
\frac{-11.54 e^{-0.6 s}}{7.01 s+1} & \frac{0.32 e^{-2.6 s}}{7.76 s+1}
\end{array}\right]
\end{aligned}
$$

This process has six possible I/O pairings. All possibilities are implemented and optimally tuned. The resulting parameters are summarized in Table 5.9.

Table 5.9 - Optimal PID Tuning Parameters for the 3x3 Distillation Column Benchmark

\begin{tabular}{c|c|c|c|c|c|c|c}
\hline $\begin{array}{c}\text { Controller } \\
\text { Parameter }\end{array}$ & Original & $\begin{array}{c}\text { Y1xU1, } \\
\text { Y2xU2, } \\
\text { Y3xU3 }\end{array}$ & $\begin{array}{l}\text { Y1xU1, } \\
\text { Y2xU3, } \\
\text { Y3xU2 }\end{array}$ & $\begin{array}{c}\text { Y1xU2, } \\
\text { Y2xU1, } \\
\text { Y3xU3 }\end{array}$ & $\begin{array}{c}\text { Y1xU2, } \\
\text { Y2xU3, } \\
\text { Y3xU1 }\end{array}$ & $\begin{array}{c}\text { Y1xU3, } \\
\text { Y2xU2, } \\
\text { Y3xU1 }\end{array}$ & $\begin{array}{c}\text { Y1xU3, } \\
\text { Y2xU1, } \\
\text { Y3xU2 }\end{array}$ \\
\hline$K_{P_{u_{1}}}$ & 1.2 & 1.5 & 2 & 0.5 & 0.001 & -0.01 & 0.11 \\
\hline$K_{I_{u_{1}}}$ & 0.24 & 0.5 & 0.5 & 0.05 & 0.001 & -0.001 & 0.11 \\
\hline$K_{D_{u_{1}}}$ & 0 & 3 & 3 & 0.5 & 0.005 & -0.01 & 0.1 \\
\hline$K_{P_{u_{2}}}$ & -0.15 & 1 & -0.008 & 1 & 0.017 & -0.5 & -0.0012 \\
\hline$K_{I_{u_{2}}}$ & -0.015 & 0.35 & $-1 \cdot 10^{-3}$ & 0.04 & 0.015 & -0.05 & -0.0001 \\
\hline$K_{D_{u_{2}}}$ & 0 & 1 & $-1 \cdot 10^{-3}$ & 1 & 0.01 & -0.1 & -0.001 \\
\hline$K_{P_{u_{3}}}$ & 0.6 & 1 & -10 & 2.5 & -10 & 75 & 50 \\
\hline$K_{I_{u_{3}}}$ & 0.15 & 0.35 & -12 & 0.5 & -12 & 7 & 10 \\
\hline$K_{D_{u_{3}}}$ & 0 & 1 & -5 & 0.5 & -5 & 5 & 10 \\
\hline
\end{tabular}

For evaluation, steps are applied to each setpoint at instants 100, 500 and 1000, and to the two disturbance variables at instants 1500 and 2000. Results are shown in Figures 5.15 and 5.16 . 

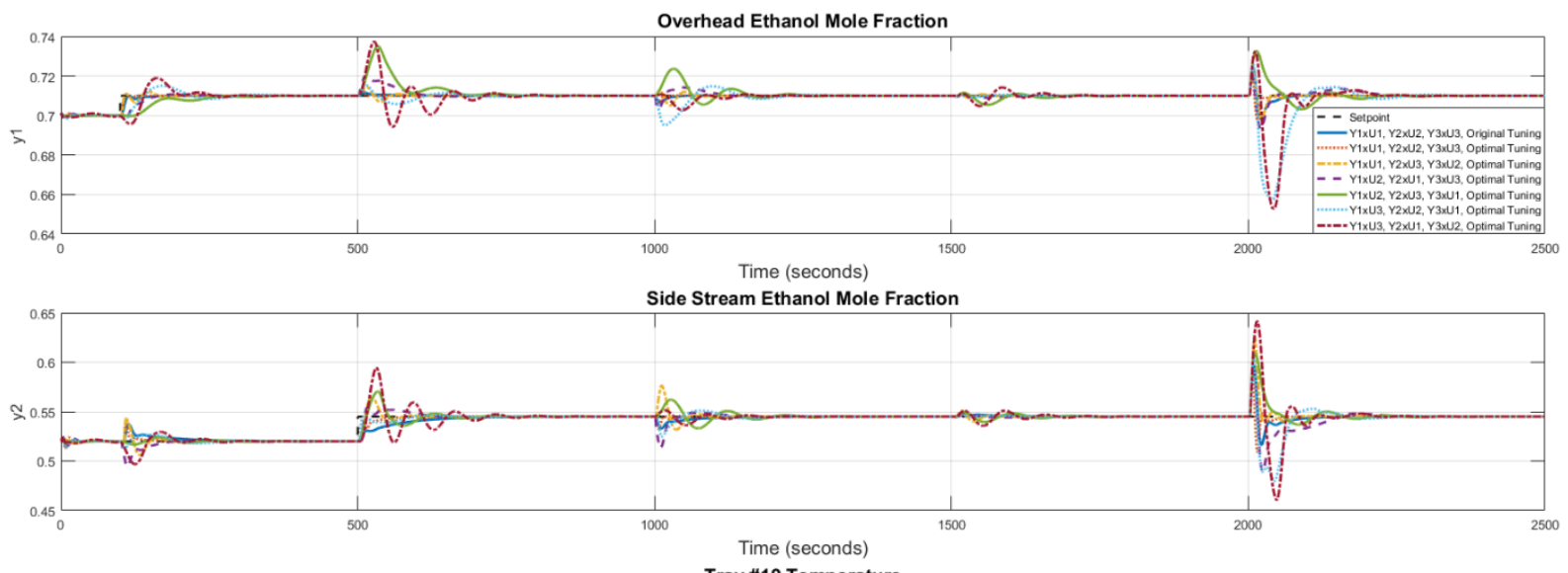

Tray \#19 Temperature

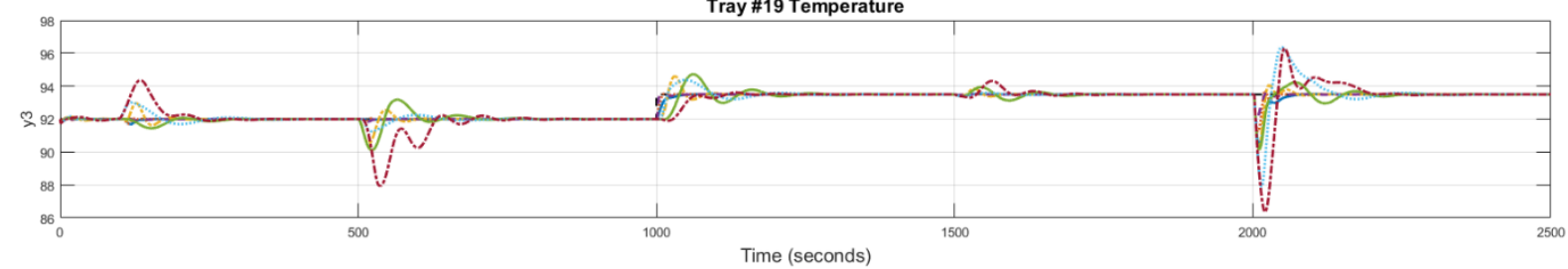

Figure 5.15 - Results for the 3×3 Distillation Column - Process Outputs.
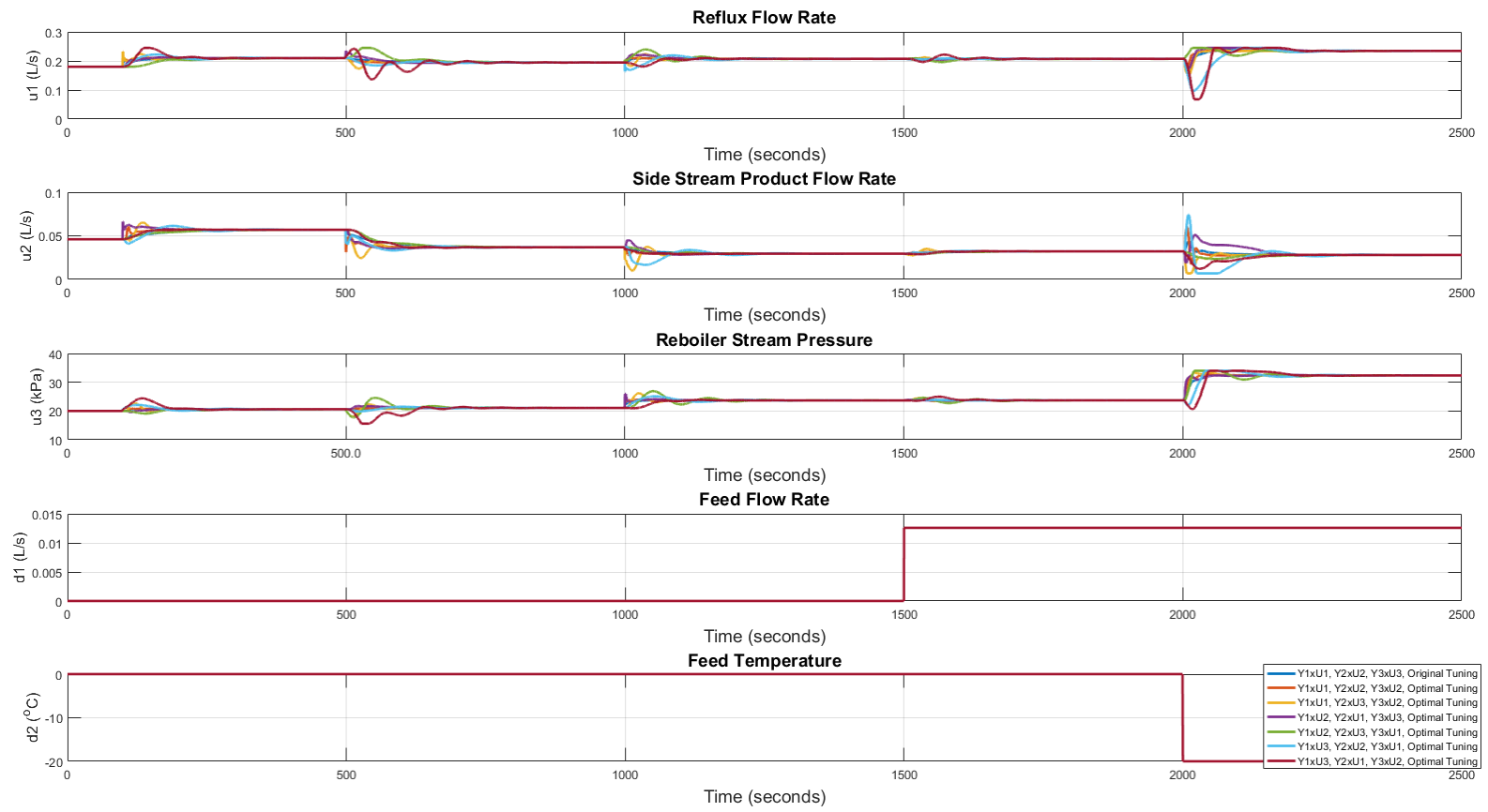

Figure 5.16 - Results for the $3 \times 3$ Distillation Column - Process Inputs.

All I/O pairing possibilities result again in acceptable controls, and the choice between one configuration or another will result in quite different dynamic responses, but the same steady-state values. Therefore, the choice of the best option depends only on dynamic performance criteria. Tables 5.10 and 5.11 summarize the corresponding performance indicators for this plant. 
Table 5.10 - Performance Indicators for the $3 \times 3$ Distillation Column Benchmark [1]

\begin{tabular}{|c|c|c|c|c|c|}
\hline $\begin{array}{l}\text { Process } \\
\text { Variable }\end{array}$ & $\begin{array}{l}\text { Performance } \\
\text { Indicator }\end{array}$ & Original & $\begin{array}{l}\text { Y1xU1, } \\
\text { Y2xU2, } \\
\text { Y3xU3 } \\
\end{array}$ & $\begin{array}{l}\text { Y1xU1, } \\
\text { Y2xU3, } \\
\text { Y3xU2 } \\
\end{array}$ & $\begin{array}{l}\text { Y1xU2, } \\
\text { Y2xU1, } \\
\text { Y3xU3 } \\
\end{array}$ \\
\hline \multirow{8}{*}{ 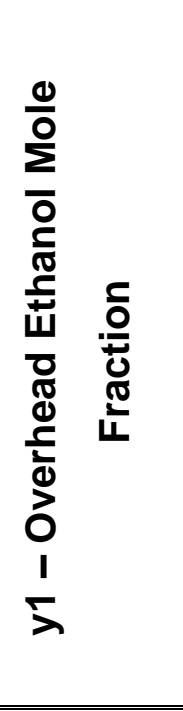 } & Settling Time (s) & 67 & 32 & 190 & 149 \\
\hline & Rise Time (s) & 11 & 7 & 6 & 53 \\
\hline & Overshoot (\%) & 0 & 13 & 22 & 7 \\
\hline & $1^{\text {st }}$ Returning Time (s) & 102 & 37 & 106 & 186 \\
\hline & $2^{\text {nd }}$ Returning Time (s) & 44 & 14 & 98 & 182 \\
\hline & $\begin{array}{l}\text { Regulatory Returning } \\
\text { Time (s) } d_{1}\end{array}$ & 24 & 20 & 66 & 128 \\
\hline & $\begin{array}{l}\text { Regulatory Returning } \\
\text { Time (s) } d_{2}\end{array}$ & 76 & 48 & 90 & 241 \\
\hline & Maximum Deviation & 0.015 & 0.012 & 0.69 & 0.69 \\
\hline \multirow{8}{*}{ 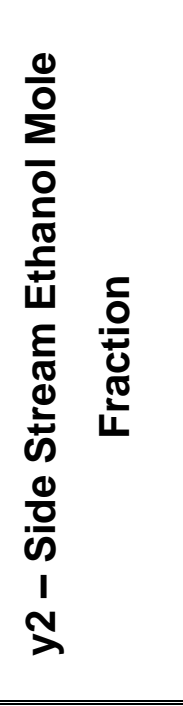 } & Settling Time (s) & 231 & 83 & 102 & 132 \\
\hline & Rise Time (s) & 134 & 45 & 12 & 12 \\
\hline & Overshoot (\%) & 0 & 0 & 79.6 & 38.8 \\
\hline & $1^{\text {st }}$ Returning Time (s) & 366 & 200 & 235 & 279 \\
\hline & $2^{\text {nd }}$ Returning Time (s) & 161 & 51 & 113 & 143 \\
\hline & $\begin{array}{l}\text { Regulatory Returning } \\
\text { Time (s) } d_{1}\end{array}$ & 130 & 58 & 83 & 129 \\
\hline & $\begin{array}{l}\text { Regulatory Returning } \\
\text { Time (s) } d_{2}\end{array}$ & 1533 & 70 & 80 & 252 \\
\hline & Maximum Deviation & 0.053 & 0.042 & 0.081 & 0.056 \\
\hline \multirow{8}{*}{ 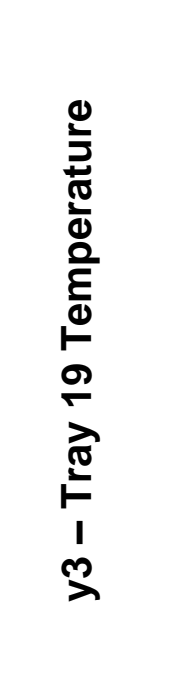 } & Settling Time (s) & 47 & 25 & 108 & 34 \\
\hline & Rise Time (s) & 31 & 9 & 18 & 4 \\
\hline & Overshoot (\%) & 0.7 & 2.7 & 74 & 2.7 \\
\hline & $1^{\text {st }}$ Returning Time (s) & 46 & 25 & 202 & 152 \\
\hline & $2^{\text {nd }}$ Returning Time (s) & 28 & 31 & 119 & 25 \\
\hline & $\begin{array}{l}\text { Regulatory Returning } \\
\text { Time (s) } d_{1}\end{array}$ & 42 & 33 & 76 & 8 \\
\hline & $\begin{array}{l}\text { Regulatory Returning } \\
\text { Time (s) } d_{2}\end{array}$ & 74 & 44 & 97 & 60 \\
\hline & Maximum Deviation & 3.0 & 2.3 & 3.3 & 1.7 \\
\hline
\end{tabular}


Table 5.11 - Performance Indicators for the 3x3 Distillation Column Benchmark [2]

\begin{tabular}{|c|c|c|c|c|}
\hline $\begin{array}{l}\text { Process } \\
\text { Variable }\end{array}$ & $\begin{array}{l}\text { Performance } \\
\text { Indicator }\end{array}$ & $\begin{array}{l}\text { Y1xU2, } \\
\text { Y2xU3, } \\
\text { Y3xU1 }\end{array}$ & $\begin{array}{l}\text { Y1xU3, } \\
\text { Y2xU2, } \\
\text { Y3xU1 } \\
\end{array}$ & $\begin{array}{l}\text { Y1xU3, } \\
\text { Y2xU1, } \\
\text { Y3xU2 } \\
\end{array}$ \\
\hline \multirow{8}{*}{ 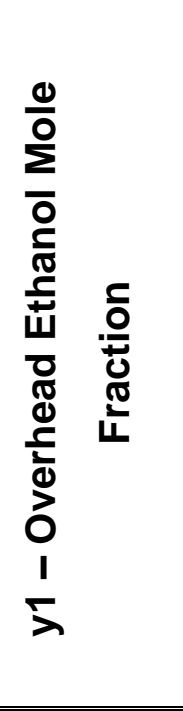 } & Settling Time (s) & 180 & 193 & 186 \\
\hline & Rise Time (s) & 150 & 36 & 39 \\
\hline & Overshoot (\%) & 0 & 50 & 89 \\
\hline & $1^{\text {st }}$ Returning Time (s) & 221 & 257 & 341 \\
\hline & $2^{\text {nd }}$ Returning Time (s) & 285 & 292 & 234 \\
\hline & $\begin{array}{l}\text { Regulatory Returning } \\
\text { Time (s) } d_{1}\end{array}$ & 268 & 128 & 265 \\
\hline & $\begin{array}{l}\text { Regulatory Returning } \\
\text { Time (s) } d_{2}\end{array}$ & 305 & 335 & 302 \\
\hline & Maximum Deviation & 0.025 & 0.053 & 0.057 \\
\hline \multirow{8}{*}{ 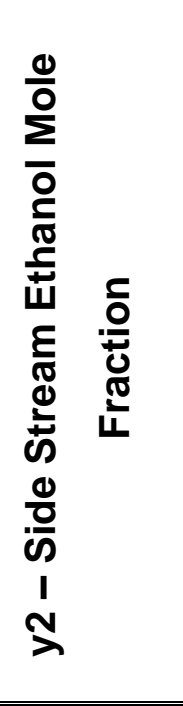 } & Settling Time (s) & 201 & 91 & 312 \\
\hline & Rise Time (s) & 13 & 8 & 15 \\
\hline & Overshoot (\%) & 102.0 & 6.8 & 198.8 \\
\hline & $1^{\text {st }}$ Returning Time (s) & 369 & 350 & 323 \\
\hline & $2^{\text {nd }}$ Returning Time (s) & 280 & 258 & 200 \\
\hline & $\begin{array}{l}\text { Regulatory Returning } \\
\text { Time (s) } d_{1}\end{array}$ & 216 & 97 & 236 \\
\hline & $\begin{array}{l}\text { Regulatory Returning } \\
\text { Time (s) } d_{2}\end{array}$ & 284 & 302 & 269 \\
\hline & Maximum Deviation & 0.064 & 0.066 & 0.085 \\
\hline \multirow{8}{*}{ 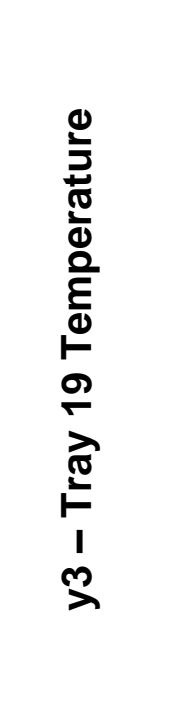 } & Settling Time (s) & 108 & 243 & 210 \\
\hline & Rise Time (s) & 37 & 17 & 60 \\
\hline & Overshoot (\%) & 82 & 58.7 & 7.3 \\
\hline & $1^{\text {st }}$ Returning Time (s) & 282 & 319 & 330 \\
\hline & $2^{\text {nd }}$ Returning Time (s) & 283 & 211 & 317 \\
\hline & $\begin{array}{l}\text { Regulatory Returning } \\
\text { Time (s) } d_{1}\end{array}$ & 243 & 133 & 244 \\
\hline & $\begin{array}{l}\text { Regulatory Returning } \\
\text { Time (s) } d_{2}\end{array}$ & 282 & 342 & 284 \\
\hline & Maximum Deviation & 3.3 & 5.6 & 7.1 \\
\hline
\end{tabular}


From the analysis of the performance indicators, the [Y1XU1, Y2xU2, Y3xU3] configuration presents better results for most of the considered indexes, being the best option for this process. Furthermore, the optimization of the controller tuning causes significant gains in all indexes when compared to the original tuning.

\subsubsection{Result Analysis}

From the simulations results, it can be noticed that all possible $\mathrm{I} / \mathrm{O}$ pairings tested can be used to build feasible controllers with all loops in automatic control, which is apparently a controversy with the widespread statement that there is a correct $1 / 0$ pairing for each process.

In fact, it was observed that there are $\mathrm{I} / \mathrm{O}$ pairings for which the control tuning is easier and classical tuning techniques can provide stable and satisfactory controllers. In other configurations, however, classical tuning approaches do not provide good results and an optimal multivariable tuning technique needs to be employed to obtain a satisfactory dynamic response. While this can be initially considered an advantage for such easier $\mathrm{I} / \mathrm{O}$ pairings, once optimal tuning technique is available, no configuration should be ruled out in order to guarantee a better final control performance.

The most important conclusion from the presented tests is that $1 / O$ pairing and control tuning are two highly intertwined problems. First, because the tuning can be employed to adjust the controllers to a given $\mathrm{I} O \mathrm{O}$ pairing in such a way that, for any $\mathrm{I} / \mathrm{O}$ pairing, an optimal tuning problem can be formulated and solved to find a feasible and optimal control for such configuration. Secondly, because choosing a best pairing should be done considering the final tuned control and the control specifications, i.e., in order to choose which $\mathrm{I} / \mathrm{O}$ pairing will be implemented, the performance of the actual control that will be obtained at the end should be observed.

In summary, better controllers can be achieved if the $\mathrm{I} / \mathrm{O}$ pairing and control tuning problems are solved in an integrated manner and if process dynamics and control behavior are considered in the $\mathrm{I} / \mathrm{O}$ pairing procedure. 


\subsubsection{Concluding Remarks}

This section presented an investigation of $\mathrm{I} / \mathrm{O}$ pairing related to independent controllers and its relationship with the control tuning.

In this study, 21 control systems were designed, implemented and evaluated for 5 different industrial benchmarks. For all possible configurations, an optimal tuning was capable of providing a stable and satisfactory control with all loops in automatic control.

The simulation results presented correspond to the application of the approach proposed in Section 4.3 with the I/O pairing solved exhaustively and the control tuning solved by optimization. This solution method evaluates all possible $\mathrm{I} / \mathrm{O}$ pairings and guarantees that the best pairing will be chosen, but it is limited to small processes.

It was verified that, despite the widespread concept of the existence of a correct I/O pairing for most plants, many different pairings can result in an acceptable behavior with the system in closed loop control. Furthermore, it was shown that the choice of the best pairing depends on the desired performance requirements and the closed loop dynamics, which greatly depends on the control tuning. So, the best I/O pairing should be determined with the corresponding best control tuning for better results.

In the next section, the techniques proposed in Chapter 4 are applied to nonsquare, larger and more complex systems.

\subsection{Applications of the Proposed Techniques}

This section presents the design of control systems for two non-square industrial benchmarks, a 3x7 heavy oil fractionator (HOF) unit (Prett \& Morari, 1987) and a 6x7 fluid catalytic cracking (FCC) unit (Grosdidier, et al., 1993). For both cases, a control system with regulatory and supervisory layers is developed. This hierarchical architecture is well accepted by the industry and widely applied in modern processes (Skogestad, 2000). The presented procedure encompass the complete control design, including the system identification, $\mathrm{I} / \mathrm{O}$ pairings, controller tuning and MPC implementation. 
The proposed Augmented Hierarchical Plantwide Control Design Technique and the proposed Optimal Multi-Objective Technique for Integrated Control Structure Selection and Tuning, both presented in Chapter 4, are applied to both chosen industrial benchmarks.

The augmented hierarchical plantwide proposed in Section 4.2 deals with the design of the complete plantwide control system, integrating system identification, control tuning and multi-objective specifications to the plantwide control design problem.

The optimal multi-objective technique for integrated control structure selection and tuning proposed in Section 4.3 integrates the tasks of $\mathrm{I} / \mathrm{O}$ pairing and controller tuning, and supports any multi-objective specification. It allows the direct use of complex specifications in the control problem, solving the $1 / O$ pairing problem simultaneously with the tuning problem.

\subsubsection{Plantwide Control Design for the HOF Benchmark}

The proposed Augmented Hierarchical Plantwide Control Design Technique is now applied to the Shell Heavy Oil Fractionator Benchmark (HOF) (Prett \& Morari, 1987), following the steps defined in Section 4.2:

I. Specification (top-down) steps:

1. Information gathering: The plant model is presented in (Prett \& Morari, 1987). The most relevant process information is described in Section 5.2.1.1.

2. General control specifications: Process specifications are presented in (Prett \& Morari, 1987). Control objectives and constraints based on such description are shown in Section 5.2.1.1, along with the controlled and manipulated variables of this process.

3. Plantwide control layer: Due to the process dimensions ( 7 controlled and 3 manipulated variables) and linearity, all optimization is performed by the MPC controller in the supervisory layer.

4. Supervisory control layer: A MPC controller is designed in Section 5.2.1.3 to determine the setpoints for the regulatory layer. 
5. Regulatory control layer: PID controllers are employed in the regulatory layer. Their design is presented in Section 5.2.1.2.

The design (bottom-up) steps of the procedure, regarding the controllers' design and evaluation, are presented in the next sections.

\subsubsection{The Shell HOF Benchmark}

The heavy oil fractionator (Prett \& Morari, 1987) is show in Figure 5.17. It has seven controlled, three manipulated and two disturbance variables.

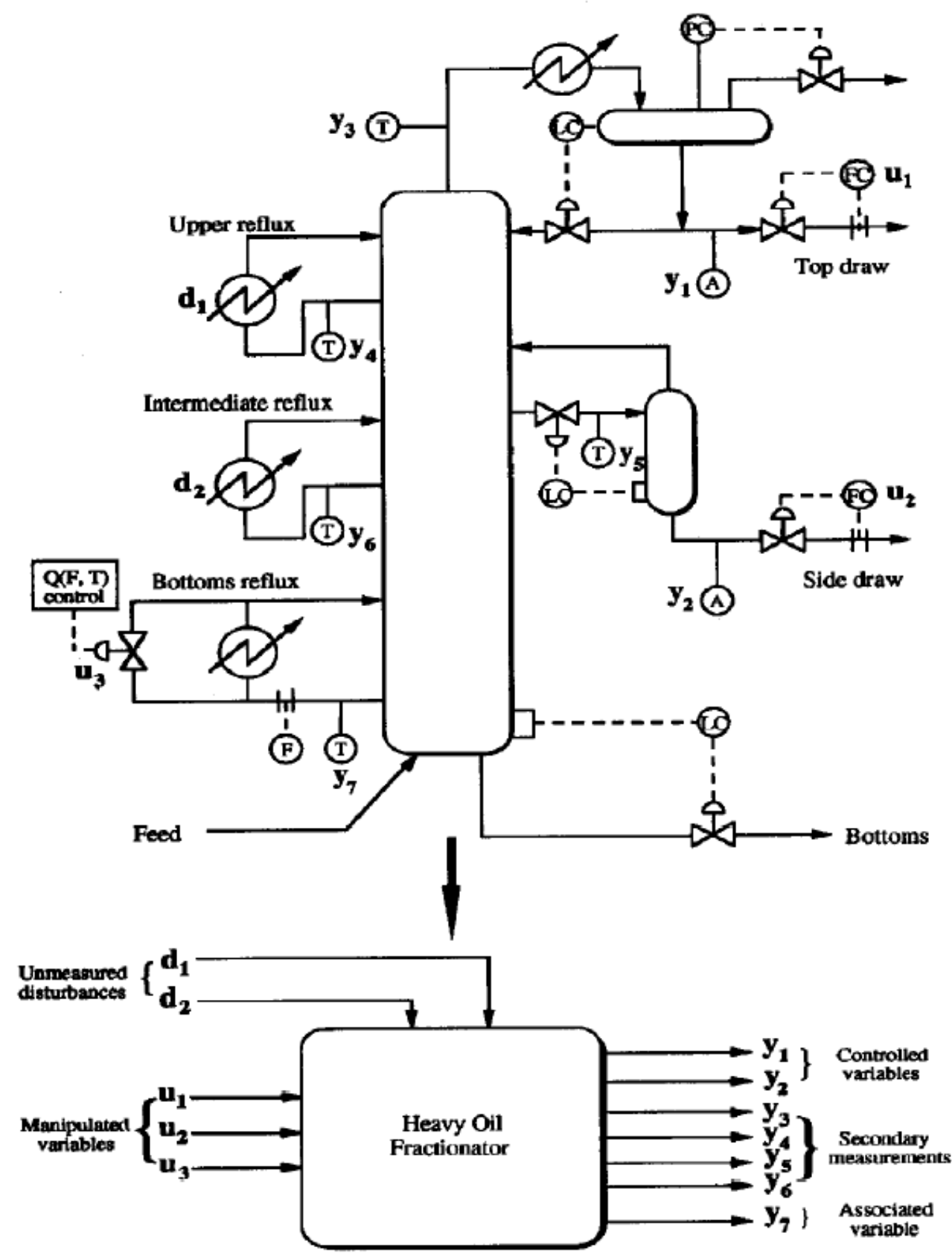

Figure 5.17 - Shell HOF and control problem (Zheng, et al., 1994). 
The main control objective for this plant is to keep the top and the side draw compositions $y_{1}$ and $y_{2}$ at their setpoints, compensating unmeasured disturbances in the upper and intermediate reflux duties $d_{1}$ and $d_{2}$. Top reflux temperature $y_{3}$, upper reflux temperature $y_{4}$, side draw temperature $y_{5}$ and intermediate reflux temperature $y_{6}$ must be kept inside a working region. Bottom reflux temperature $y_{7}$ should also be kept at its setpoint. The manipulated variables are the top drawn $u_{1}$, side drawn $u_{2}$ and reflux duty $u_{3}$.

This process is modeled by a linear and full transfer function matrix with time delays, shown in Equations (5.13), (5.14) and (5.15), in minutes.

$$
\begin{aligned}
& y(s)=G(s) \cdot u(s)+H(s) \cdot d(s) \\
& G(s)=\left[\begin{array}{ccc}
\frac{4.05 \cdot e^{-27 \cdot s}}{50 \cdot s+1} & \frac{1.77 \cdot e^{-28 \cdot s}}{60 \cdot s+1} & \frac{5.88 \cdot e^{-27 \cdot s}}{35 \cdot s+1} \\
\frac{5.39 \cdot e^{-18 \cdot s}}{50 \cdot s+1} & \frac{5.7 \cdot e^{-14 \cdot s}}{60 \cdot s+1} & \frac{6.9 \cdot e^{-15 \cdot s}}{40 \cdot s+1} \\
\frac{3.66 \cdot e^{-2 \cdot s}}{9 \cdot s+1} & \frac{1.65 \cdot e^{-20 \cdot s}}{30 \cdot s+1} & \frac{5.33 \cdot e^{-2 \cdot s}}{40 \cdot s+1} \\
\frac{5.92 \cdot e^{-11 \cdot s}}{12 \cdot s+1} & \frac{2.54 \cdot e^{-12 \cdot s}}{27 \cdot s+1} & \frac{8.10 \cdot e^{-2 \cdot s}}{20 \cdot s+1} \\
\frac{4.13 \cdot e^{-5 \cdot s}}{8 \cdot s+1} & \frac{2.38 \cdot e^{-7 \cdot s}}{19 \cdot s+1} & \frac{6.23 \cdot e^{-2 \cdot s}}{10 \cdot s+1} \\
\frac{4.06 \cdot e^{-8 \cdot s}}{13 \cdot s+1} & \frac{4.18 \cdot e^{-4 \cdot s}}{33 \cdot s+1} & \frac{6.53 \cdot e^{-1 \cdot s}}{9 \cdot s+1} \\
\frac{4.38 \cdot e^{-20 \cdot s}}{33 \cdot s+1} & \frac{4.42 \cdot e^{-22 \cdot s}}{44 \cdot s+1} & \frac{7.2}{19 \cdot s+1}
\end{array}\right] \\
& H(s)=\left[\begin{array}{cc}
\frac{1.20 \cdot e^{-27 \cdot s}}{45 \cdot s+1} & \frac{1.44 \cdot e^{-27 \cdot s}}{40 \cdot s+1} \\
\frac{1.5 \cdot e^{-15 \cdot s}}{25 \cdot s+1} & \frac{1.83 \cdot e^{-15 \cdot s}}{20 \cdot s+1} \\
\frac{1.16}{11 \cdot s+1} & \frac{1.27}{6 \cdot s+1} \\
\frac{1.73}{5 \cdot s+1} & \frac{1.79}{19 \cdot s+1} \\
\frac{1.31}{2 \cdot s+1} & \frac{1.26}{22 \cdot s+1} \\
\frac{1.19}{19 \cdot s+1} & \frac{1.17}{24 \cdot s+1} \\
\frac{1.14}{27 \cdot s+1} & \frac{1.26}{32 \cdot s+1}
\end{array}\right]
\end{aligned}
$$

Setpoint and constraint values are presented in Table 5.12. 
Table 5.12 - Variable constraints for the Shell HOF (Zheng, et al., 1994).

\begin{tabular}{l|c|c|c}
\hline \multicolumn{1}{c|}{ Variable } & Nominal & Minimum & Maximum \\
\hline$y_{1}$ & 0 & -0.5 & 0.5 \\
\hline$y_{2}$ & 0 & - & - \\
\hline$y_{3}$ & - & -1 & 1 \\
\hline$y_{4}$ & - & -1 & 1 \\
\hline$y_{5}$ & - & -1 & 1 \\
\hline$y_{6}$ & - & -1 & 1 \\
\hline$y_{7}$ & -0.5 & -0.5 & - \\
\hline$u_{1}$ & - & -0.5 & 0.5 \\
\hline$u_{2}$ & - & -0.5 & 0.5 \\
\hline$u_{3}$ & - & -0.5 & 0.5 \\
\hline$d_{1}$ (expected) & 0 & -0.5 & 0.5 \\
\hline$d_{2}$ (expected) & 0 & -0.5 & 0.5 \\
\hline
\end{tabular}

\subsubsection{Regulatory Control Layer Design}

The regulatory control layer design follows the steps defined in the proposed Augmented Hierarchical Plantwide Control Design Technique, and employs the proposed Optimal Multi-Objective Technique for Integrated Control Structure Selection and Tuning to define $\mathrm{I} / \mathrm{O}$ pairing and optimal control tuning.

For the control design and tuning, the process model given in (Zheng, et al., 1994) is employed.

The first step in the design is the selection of the controlled variables. With three manipulated variables available, three variables can be controlled by independent PID controllers. Based on the control specifications, $y_{1}, y_{2}$ and $y_{7}$ are straightforward choices for controlled variables.

The next step is to determine the $\mathrm{I} / \mathrm{O}$ pairing and controllers tuning. The process model and PID controllers are implemented in Simulink ${ }^{\circledR}$ and the resulting optimization problem is solved with the Genetic Algorithm from MATLAB ${ }^{\circledR}$.

All the PID controllers are implemented in the digital form with sampling time of 1 second (the original model is appropriately adjusted), with manipulated variables limited according to the specified ranges. Controllers are tuned minimizing settling time and rise time for servo and regulatory modes, keeping the overshoot and undershoot constrained, in this order, prioritizing the controlled variables from $y_{1}$ to $y_{2}$ and $y_{7}$. 
For this setup, there are six possible $\mathrm{I} / \mathrm{O}$ pairings. The pairing is determined simultaneously with the optimal tuning for the PID controllers. Differently from the examples in Section 5.1, a complete optimization approach is employed, rather than exhaustively testing all possible pairings. The two best pairings and tunings are shown in Table 5.13. Step responses for these configurations are presented in Figure 5.18, with steps applied to each setpoint and disturbance variable at an interval of 12 hours.

Table 5.13 - Optimal PID Tuning Parameters for the Shell HOF Benchmark.

\begin{tabular}{c|c|c}
\hline Controller Parameter & Configuration 1 & Configuration 2 \\
\hline Pairing $y_{1}$ & $u_{1}$ & $u_{2}$ \\
\hline Pairing $y_{2}$ & $u_{2}$ & $u_{1}$ \\
\hline Pairing $y_{7}$ & $u_{3}$ & $u_{3}$ \\
\hline$K_{P_{u_{1}}}$ & 0.2 & -0.15 \\
\hline$K_{I_{u_{1}}}$ & 0.0003 & -0.000075 \\
\hline$K_{D_{u_{1}}}$ & 0.2 & -0.5 \\
\hline$K_{P_{u_{2}}}$ & 0.3 & 0.3 \\
\hline$K_{I_{u_{2}}}$ & 0.0003 & 0.0003 \\
\hline$K_{D_{u_{2}}}$ & 0.3 & 0.3 \\
\hline$K_{P_{u_{3}}}$ & 3 & 3 \\
\hline$K_{I_{u_{3}}}$ & 0.03 & 0.03 \\
\hline$K_{D_{u_{3}}}$ & 0.1 & 0.1 \\
\hline
\end{tabular}
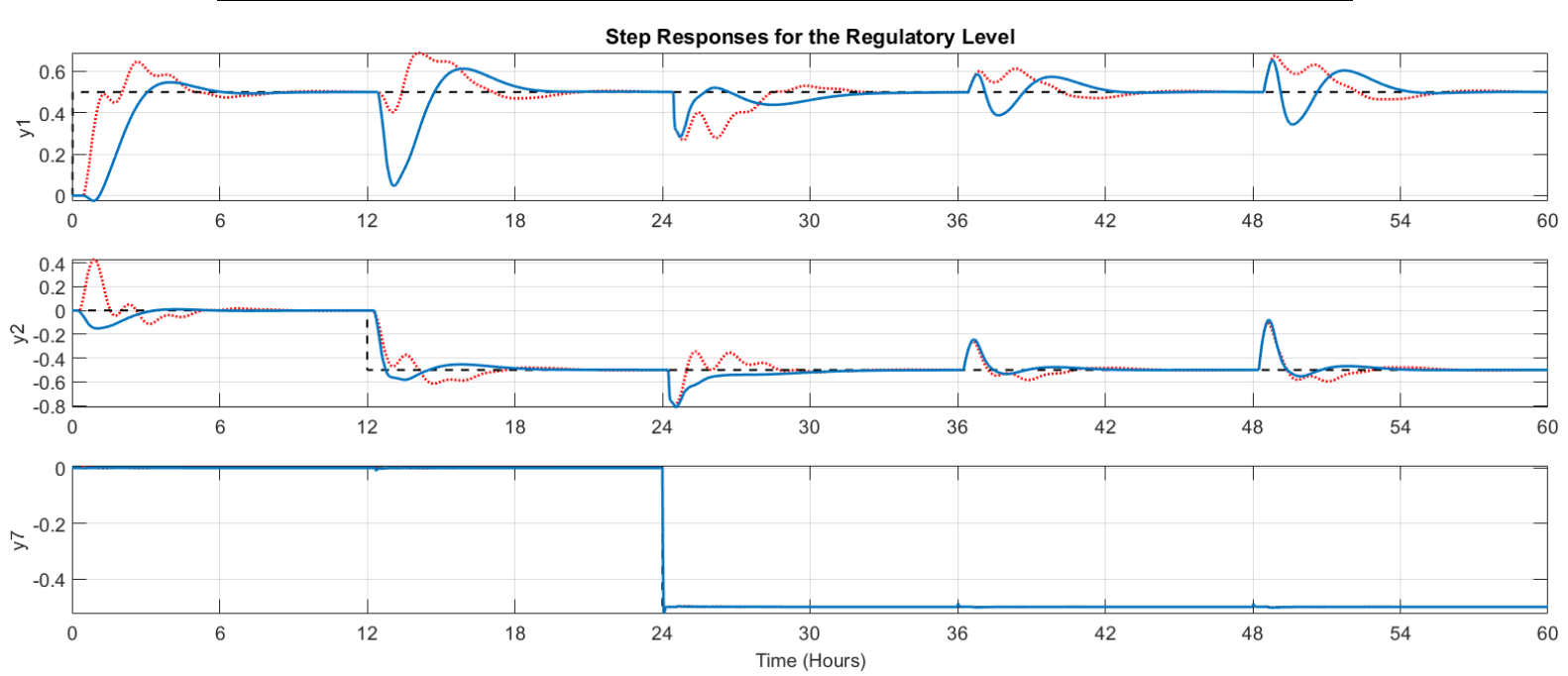

Figure 5.18 - PID control. Configurations 1 (red) and 2 (blue).

It can be noted that both configurations satisfy the control requirements. The corresponding performance indexes are presented in Table 5.14. 
Table 5.14 - Performance Indicators for the Regulatory Control of the Shell HOF Benchmark.

\begin{tabular}{|c|c|c|c|}
\hline $\begin{array}{l}\text { Process } \\
\text { Variable }\end{array}$ & Performance Indicator & $\begin{array}{c}\text { Y1xU1, Y2xU2, } \\
\text { Y7xU3 }\end{array}$ & $\begin{array}{c}\text { Y1xU2, Y2xU1, } \\
\text { Y7xU3 }\end{array}$ \\
\hline \multirow{8}{*}{ 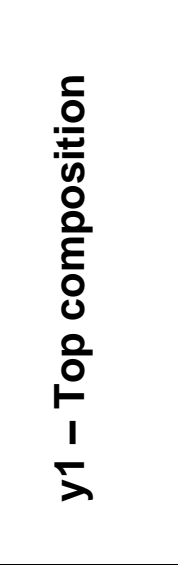 } & Settling Time $(\mathrm{h})$ & 7.8 & 5.6 \\
\hline & Rise Time (h) & 1.1 & 2.7 \\
\hline & Overshoot (\%) & 29.2 & 9.4 \\
\hline & $1^{\text {st }}$ Returning Time $(\mathrm{h})$ & 7.9 & 6.7 \\
\hline & $2^{\text {nd }}$ Returning Time (h) & 7.5 & 8.0 \\
\hline & Regulatory Returning Time $(\mathrm{h}) d_{1}$ & 7.1 & 6.0 \\
\hline & Regulatory Returning Time $(\mathrm{h}) d_{2}$ & 7.2 & 6.0 \\
\hline & Maximum Deviation & 0.19 & 0.46 \\
\hline \multirow{8}{*}{ 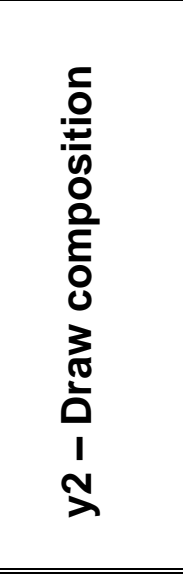 } & Settling Time $(\mathrm{h})$ & 5.3 & 6.4 \\
\hline & Rise Time (h) & 0.9 & 0.7 \\
\hline & Overshoot (\%) & 23.1 & 16.4 \\
\hline & $1^{\text {st }}$ Returning Time $(\mathrm{h})$ & 7.4 & 3.1 \\
\hline & $2^{\text {nd }}$ Returning Time (h) & 7.0 & 7.2 \\
\hline & Regulatory Returning Time $(\mathrm{h}) d_{1}$ & 7.1 & 5.4 \\
\hline & Regulatory Returning Time $(\mathrm{h}) d_{2}$ & 7.3 & 5.5 \\
\hline & Maximum Deviation & 0.4 & 0.4 \\
\hline \multirow{8}{*}{ 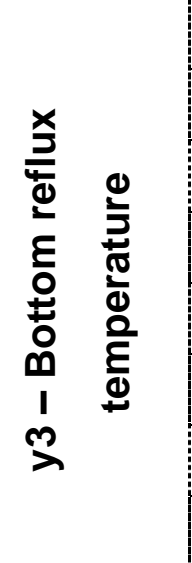 } & Settling Time $(\mathrm{h})$ & 0.1 & 0.1 \\
\hline & Rise Time (h) & 0.04 & 0.04 \\
\hline & Overshoot (\%) & 5.0 & 5.0 \\
\hline & $1^{\text {st }}$ Returning Time $(\mathrm{h})$ & 0 & 0 \\
\hline & $2^{\text {nd }}$ Returning Time (h) & 0 & 0.3 \\
\hline & Regulatory Returning Time $(\mathrm{h}) d_{1}$ & 0.03 & 0.03 \\
\hline & Regulatory Returning Time $(\mathrm{h}) d_{2}$ & 0.03 & 0.03 \\
\hline & Maximum Deviation & 0.01 & 0.01 \\
\hline
\end{tabular}

Both configurations perform well for $y_{3}$, but Configuration 2 presents smoother responses and better settling times for $y_{1}$ and $y_{2}$, being chosen for the PID layer. Configuration 1 is a more intuitive pairing, due to the proximity of the controlled and the manipulated variables, however, Configuration 2 results slightly better due to the I/O variable coupling. 
It is relevant to emphasize that the PID tunings are not optimal for each variable individually, but for all the controlled variables in a multivariable manner, i.e., the employed approach takes into consideration the interference among the loops to optimize the tunings. Furthermore, not only the standard step responses are optimized, but also the disturbance rejection to achieve an overall optimal response.

\subsubsection{Supervisory Control Layer Design}

With the PID controllers set, the MPC layer must be designed. This layer generates setpoints for the PID layer to optimize trajectories and keep the process under operational constraints. For this study, the MATLAB ${ }^{\circledR}$ MPC is employed.

The first and most important step in the MPC design is to obtain a model for the process in closed loop with the PID control layer. This is performed using System Identification.

The process is identified following the procedure and techniques described in (Garcia, et al., 2014). First, the system is excited in an identification experiment. The data collected is then organized and several models are obtained for the outputs, employing different identification algorithms and model orders. Finally, all models are evaluated and compared with the process step responses, being the models with best fitness for each output chosen.

The best MISO models are grouped to compose a MIMO model to be embedded in the MPC. The final model and process step responses for the three PID setpoints are presented in Figure 5.19. This model is considered satisfactory to describe the process, even with some mismatches in some of the dynamics.

The next step in the design of the MPC layer is to build and tune the MPC controller. A main feature of this controller is to allow the use of explicit constraints, also, since the MPC controller is capable of controlling all seven process variables, a variable pairing is not needed for this layer.

A sampling time of 1 minute is specified for the MPC layer. For the other control parameters, the same tuning problem of the PID control layer is applied and solved with the optimal control tuning technique. The resulting parameters are presented in Table 5.15. 


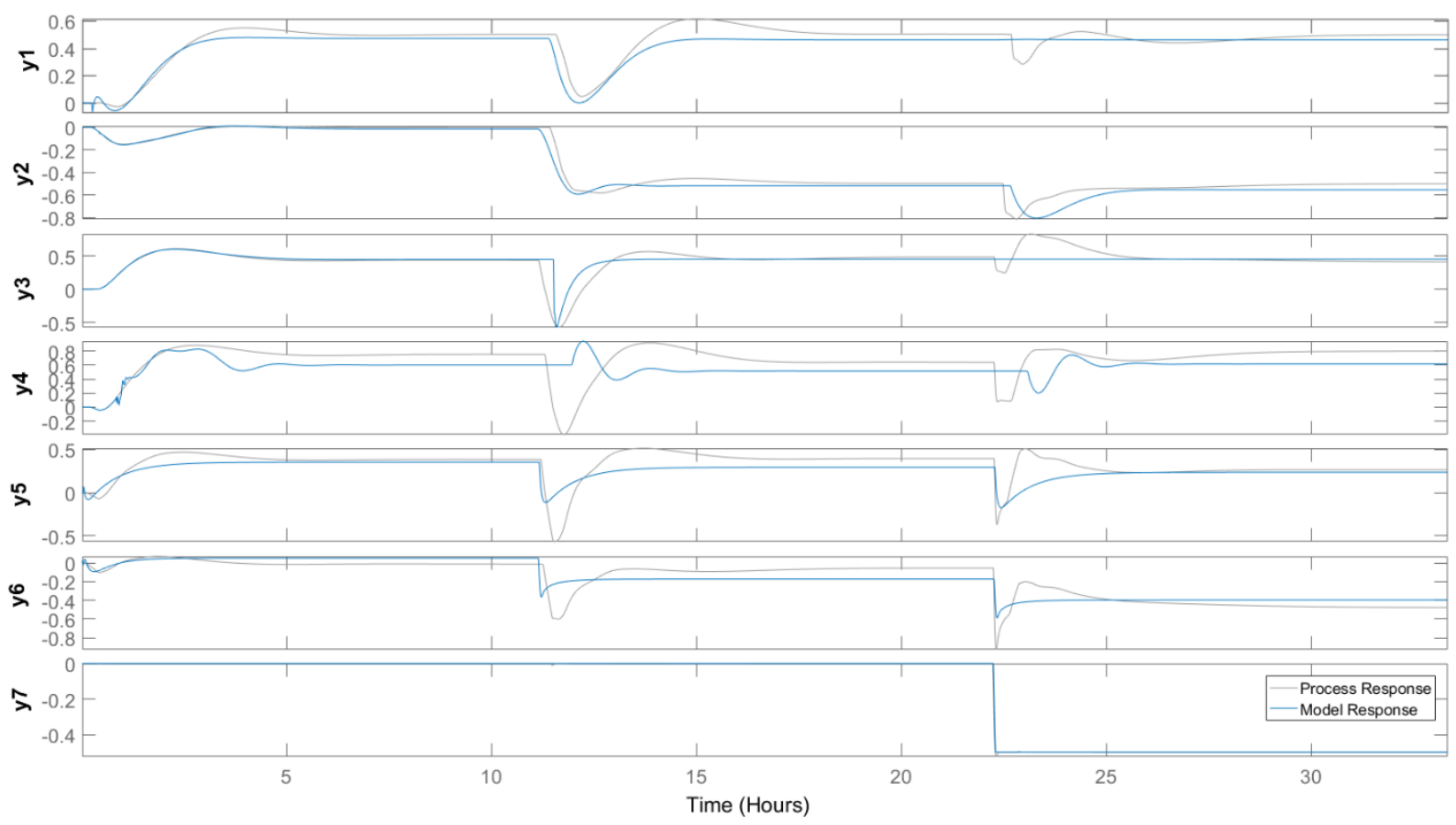

Figure 5.19 - Identified model for the HOF Plant in closed loop with PID controllers.

Table 5.15 - Optimal MPC Tuning Parameters for the Shell HOF Benchmark.

\begin{tabular}{l|c}
\hline \multicolumn{1}{c|}{ Controller Parameter } & Value \\
\hline Sampling Time (s) & 60 \\
\hline Prediction Horizon (steps) & 120 \\
\hline Control Horizon (steps) & 10 \\
\hline$y_{1}$ Weight & 1 \\
\hline$y_{2}$ Weight & 1.5 \\
\hline$y_{3}$ Weight & 0 \\
\hline$y_{4}$ Weight & 0 \\
\hline$y_{5}$ Weight & 0 \\
\hline$y_{6}$ Weight & 0 \\
\hline$y_{7}$ Weight & 3 \\
\hline$S P_{1}$ Weight & 0 \\
\hline$S P_{2}$ Weight & 0 \\
\hline$S P_{7}$ Weight & 0 \\
\hline$\Delta S P_{1}$ Weight & 0.1 \\
\hline$\Delta S P_{2}$ Weight & 0.1 \\
\hline$\Delta S P_{7}$ Weight & 0.1 \\
\hline
\end{tabular}

With the MPC layer designed, the Shell HOF benchmark is controlled by a PID layer operating at 1 second sampling time and an MPC layer operating with sampling time of 1 minute, that generates setpoints for the PID controllers. 
The same steps employed to evaluate the PID controllers are applied to the MPC layer. Results for the process controlled by the two layers of controllers and also by only the PID layer are presented in Figure 5.20.

As can be noted, both control systems follow the specification. For the tested scenarios, the system with only a PID layer performs better than the architecture with the MPC layer, and with smoother responses. This worse behavior of the MPC is attributed to the low controller robustness face to model uncertainties, which can be seen in Figure 5.19.

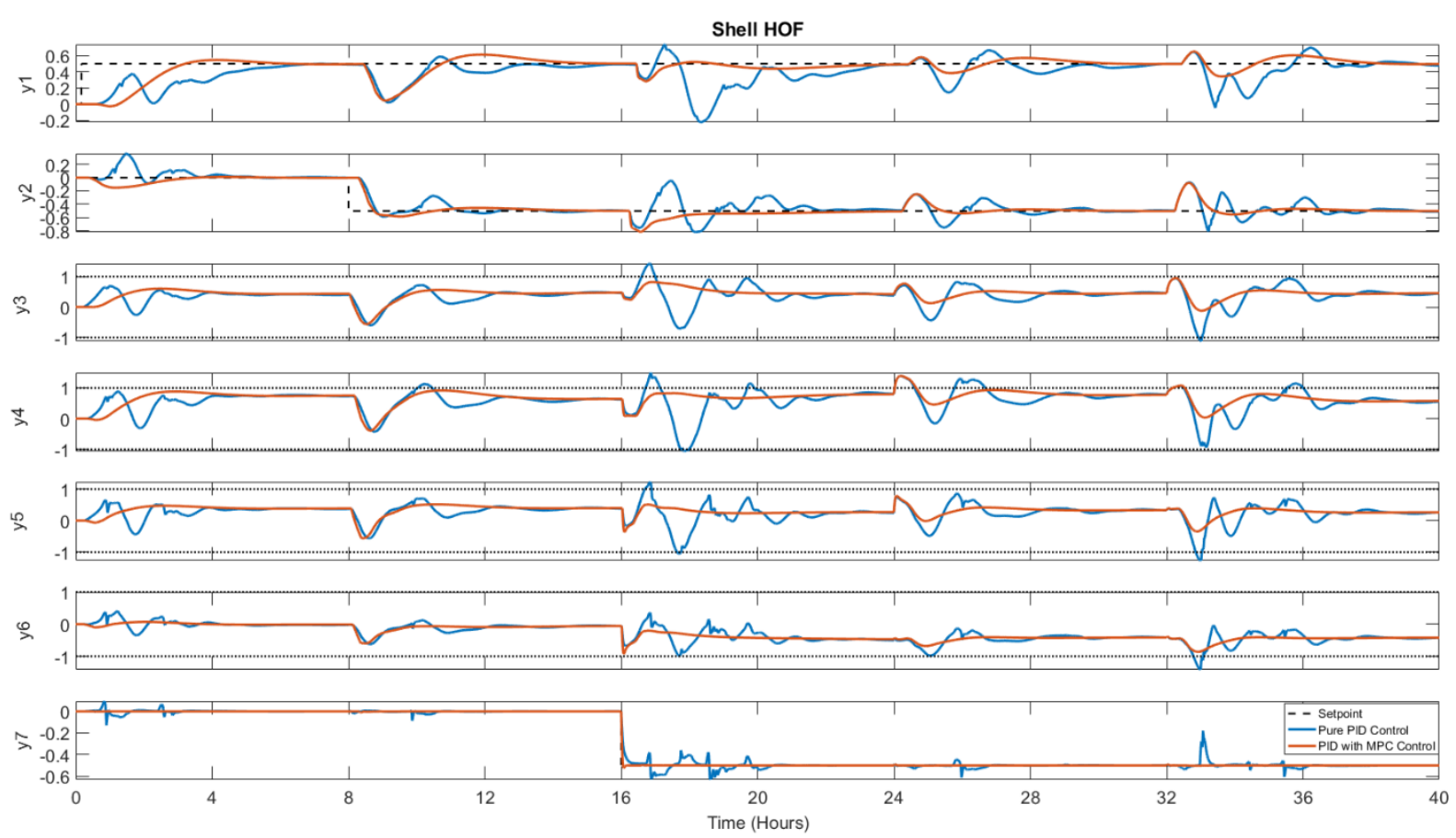

Figure 5.20 - Results for the Shell HOF Benchmark.

Table 5.16 presents the SSE (Sum of Squared Errors) performance index for both architectures.

Table 5.16 - SSE Performance Indexes for the Shell HOF.

\begin{tabular}{c|cc}
\hline Variable & SSE for PID Controllers & SSE for MPC with PID Controllers \\
\hline$y_{1}$ & 2664.9 & 5412.3 \\
\hline$y_{2}$ & 1187.4 & 2299.0 \\
\hline$y_{3}$ & 32006.7 & 33283.8 \\
\hline$y_{4}$ & 74807.1 & 69710.1 \\
\hline$y_{5}$ & 17241.2 & 21471.0 \\
\hline$y_{6}$ & 18958.4 & 21775.9 \\
\hline$y_{7}$ & 11.2 & 125.2 \\
\hline
\end{tabular}




\subsubsection{Results for the Shell HOF Benchmark}

In this final step of the plantwide design procedure, the results are analyzed and the design decisions are reviewed and adjusted.

It was noted that the MPC layer did not improve the overall system performance, and even caused some excessive oscillations in the process, which was caused by the low robustness of the employed controller (the MATLAB ${ }^{\circledR}$ MPC) face to model uncertainties. Based on this, the MPC layer is discarded for this process, and the final control system is composed only by the PID layer, whose controllers are collaborative due to the multivariable tuning performed.

It is relevant to emphasize that the focus of this work is the plantwide control design, and not the evaluation of the specific control algorithms. Although there are other MPC algorithms that could provide better results for the supervisory layer, the objective here is to show that the one employed provided bad results, and that the plantwide design technique can be employed to evaluate the control system and to assess the benefits and need of an MPC layer.

\subsubsection{Plantwide Control Design for the FCC Benchmark}

The proposed Augmented Hierarchical Plantwide Control Design Technique is now applied to the fluid catalytic cracking (FCC) unit (Grosdidier, et al., 1993), following the steps defined in Section 4.2:

I. Specification (top-down) steps:

1. Information gathering: The plant model is presented in (Grosdidier, et al., 1993). The process is briefly described in Section 5.2.2.1.

2. General control specifications: Process specifications are presented in (Grosdidier, et al., 1993). Control objectives and constraints based on such description are also show in Section 5.2.2.1, along with the controlled and manipulated variables of this process.

3. Plantwide control layer: All optimization is performed by the MPC controller in the supervisory layer. 
4. Supervisory control layer: An MPC controller is designed in Section 5.2.2.3 to determine the setpoints for the regulatory layer. This MPC shall include all process variables and optimize the overall plant performance.

5. Regulatory control layer: PID controllers are employed in the regulatory layer. Their design is presented in Section 5.2.2.2. With six available manipulated variables, six PID controllers should compose this layer.

The design (bottom-up) steps of the procedure, regarding the controllers design and evaluation, are presented in the next sections.

\subsubsection{The FCC Benchmark}

The FCC unit is presented in Figure 5.21 (Grosdidier, et al., 1993).

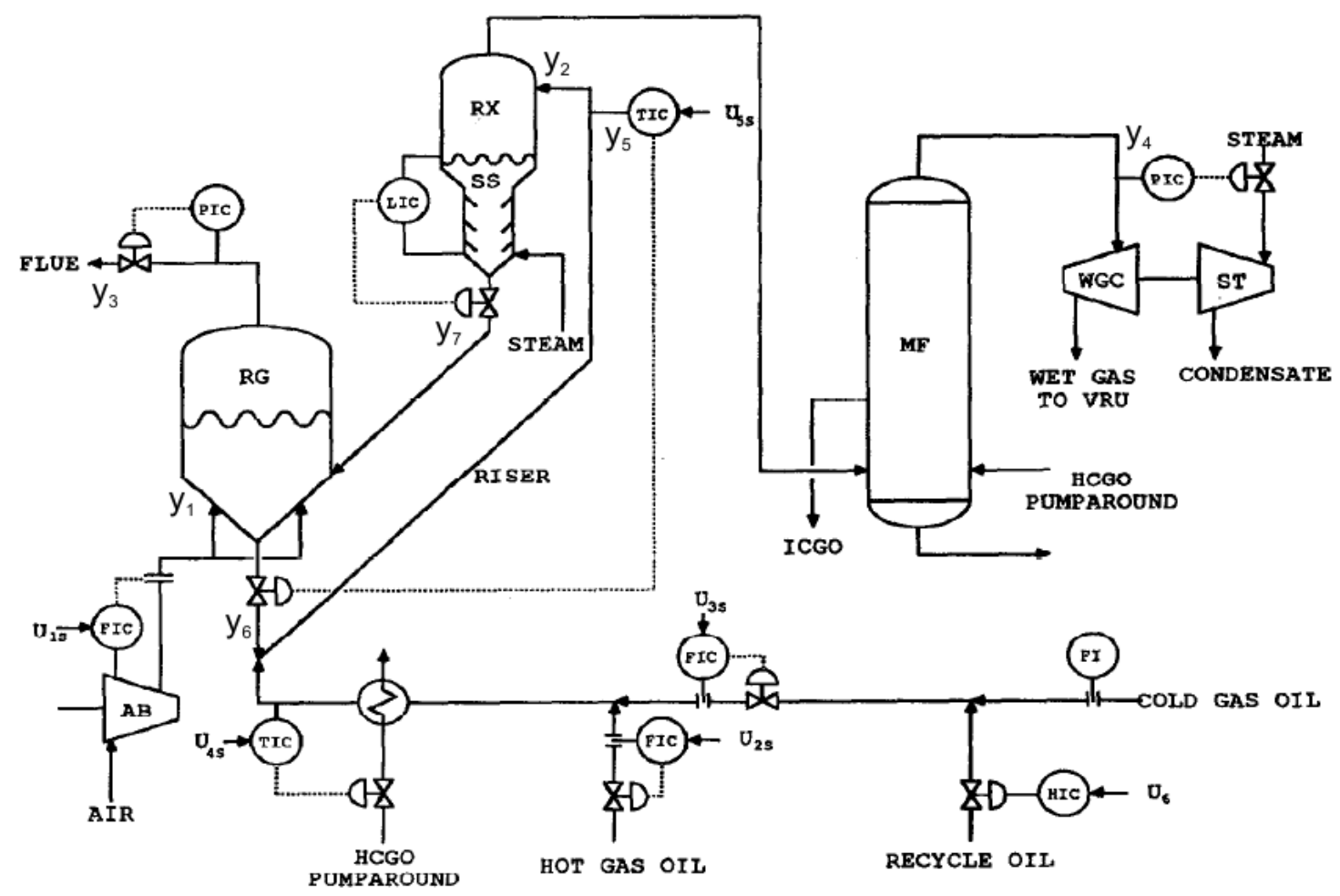

Figure 5.21 - FCC benchmark (Grosdidier, et al., 1993).

This model has six input variables and seven output variables. Plant outputs are the flue gas $O_{2}$ concentration $\left(y_{1}\right)$, regenerator bed temperature $\left(y_{2}\right)$, fuel gas flow $\left(y_{3}\right)$, wet gas compressor suction pressure controller output $\left(y_{4}\right)$, riser outlet temperature $\left(y_{5}\right)$, regenerator catalyst slide valve pressure differential $\left(y_{6}\right)$ and spent catalyst slide valve pressure differential $\left(y_{7}\right)$. The manipulated variables are combustion air flow $\left(u_{1}\right)$, hot gas oil flow $\left(u_{2}\right)$, combined cold gas and recycle oils $\left(u_{3}\right)$, feed preheat temperature 
$\left(u_{4}\right)$, riser outlet temperature $\left(u_{5}\right)$ and recycle oil flow controller output $\left(u_{6}\right)$. Process variables and control specifications are shown in Table 5.17. Further details can be found in (Grosdidier, et al., 1993).

Table 5.17 - Variable constraints for the FCC benchmark (Grosdidier, et al., 1993).

\begin{tabular}{l|c|c|c}
\hline Variable & Nominal & Minimum & Maximum \\
\hline$y_{1}(\%)$ & 1 & - & - \\
\hline$y_{2}\left({ }^{\circ} \mathrm{C}\right)$ & 711 & 705 & 735 \\
\hline$y_{3}(\mathrm{~T} / \mathrm{s})$ & 0.0043 & 0 & 0.5 \\
\hline$y_{4}(\%)$ & 60 & 0 & 70 \\
\hline$y_{5}(\%)$ & 70 & 0 & 80 \\
\hline$y_{6}(\mathrm{kPa})$ & 32 & 22 & - \\
\hline$y_{7}(\mathrm{kPa})$ & 20 & 0 & 24 \\
\hline$u_{1}(\mathrm{~T} / \mathrm{h})$ & 154.3 & 140 & 155 \\
\hline$u_{2}\left(\mathrm{~m}^{3} / \mathrm{h}\right)$ & 100 & 90 & 110 \\
\hline$u_{3}\left(\mathrm{~m}^{3} / \mathrm{h}\right)$ & 100 & 90 & 110 \\
\hline$u_{4}\left({ }^{\circ} \mathrm{C}\right)$ & 240 & 230 & 250 \\
\hline$u_{5}\left({ }^{\circ} \mathrm{C}\right)$ & 520 & 515 & 535 \\
\hline$u_{6}(\%)$ & 50 & 20 & 80 \\
\hline
\end{tabular}

This process is modeled by a linear and full transfer function matrix with time delays, shown in Equations (5.16), (5.17) and (5.18), in which $C(s)$ represents local controllers. Equations are expressed in minutes.

$$
\begin{aligned}
& y(s)=G(s) \cdot C(s) \cdot u(s)
\end{aligned}
$$

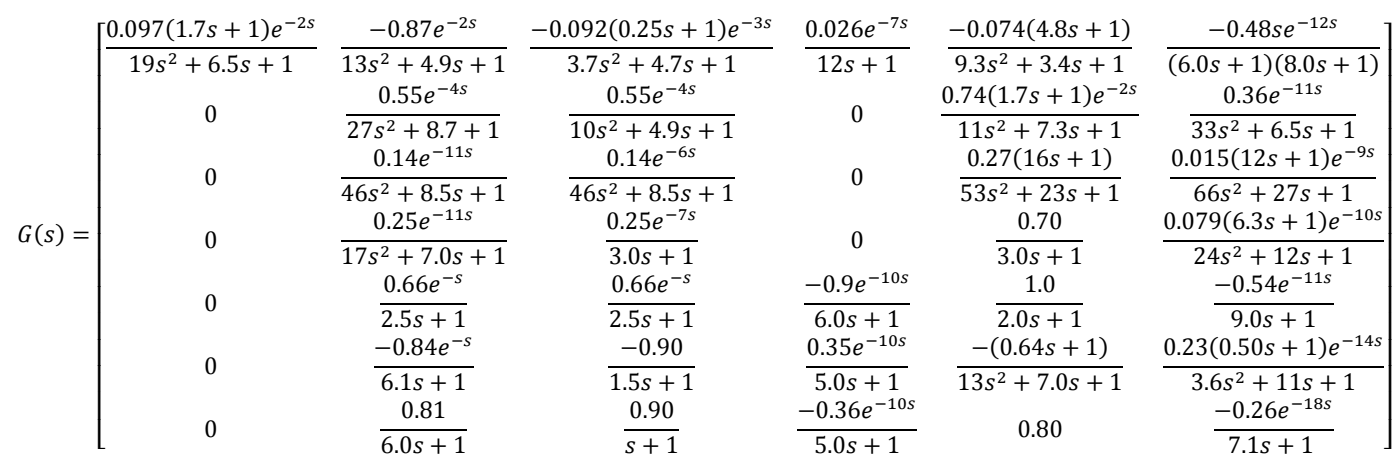

$$
\begin{aligned}
& C(s)=\left[\begin{array}{cccccc}
\frac{1}{(0.75 s+1)(4.5 s+1)} & 0 & 0 & 0 & 0 & 0 \\
0 & \frac{1}{s+1} & 0 & 0 & 0 & 0 \\
0 & 0 & \frac{1}{1.7 s^{2}+2.1 s+1} & 0 & 0 & 0 \\
0 & 0 & 0 & \frac{(3.3 s+1) e^{-s}}{40 s^{2}+13 s+1} & 0 & 0 \\
0 & 0 & 0 & 0 & \frac{0.64 s+1}{13 s^{2}+7.0 s+1} & 0 \\
0 & 0 & 0 & 0 & 0 & 1
\end{array}\right]
\end{aligned}
$$




\subsubsection{Regulatory Control Layer Design}

As for the previous benchmark, the regulatory control layer is designed following the steps defined by the Augmented Hierarchical Plantwide Control Design Technique, and employing the Optimal Multi-Objective Technique for Integrated Control Structure Selection and Controller Tuning.

The first task in the control design is the selection of the controlled variables. For this process, this choice is not quite straightforward as it was in the previous example. To determine which variables to control, the problem in the form (4.1) is built first with a single objective to minimize the overall deviation from the setpoints for all variables, i.e., a control that keeps most variables at their setpoints is sought.

The plant model and the six PID controllers are implemented in Simulink ${ }^{\circledR}$, with the PID in digital form with sampling time of 1 second (the model parameters are appropriately adjusted). Each controller is associated with a manipulated variable and includes limits to keep variables inside operational ranges. The resulting problem is solved with Genetic Algorithm from MATLAB ${ }^{\circledR}$, which varies the controlled variables associated with each controller and the PID tunings. The solution of this first problem shows that it is best to control $y_{1}, y_{2}, y_{4}, y_{5}$ and $y_{6}$, keeping the loops open for $y_{3}$ and $y_{7}$ and keeping $u_{2}$ constant, since this process input have a strong influence in many outputs and should be better used by a multivariable controller in the upper layer.

A second optimal control design problem is then formulated to refine the $1 / 0$ pairing and PID tunings. For this second problem, multiple objectives are stablished. First, the settling and rise times for step responses in $y_{1}$ are optimized with highest priority, followed by the maximum deviation and returning time of $y_{1}$ to its setpoint when it is disturbed by a setpoint change in other variables. The same indexes are then optimized for $y_{2}, y_{4}, y_{5}$ and $y_{6}$, in this order.

The final I/O pairings and tunings obtained, optimized for an overall optimal response, are shown in Table 5.18, and the respective response in Figure 5.22. The optimization produces good results, with the PID controllers performing satisfactorily. Performance indicators are shown and compared with the dual layer configuration in the next section. 
Table 5.18 - Optimal PID Tuning Parameters for the FCC Benchmark.

\begin{tabular}{l|c}
\hline Controller Parameter & Value \\
\hline Pairing $y_{1}$ & $u_{1}$ \\
\hline Pairing $y_{2}$ & $u_{6}$ \\
\hline Pairing $y_{4}$ & $u_{5}$ \\
\hline Pairing $y_{5}$ & $u_{4}$ \\
\hline Pairing $y_{6}$ & $u_{3}$ \\
\hline$K_{P_{u_{1}}}$ & $2.2 \cdot 10^{-3}$ \\
\hline$K_{I_{u_{1}}}$ & $4 \cdot 10^{-6}$ \\
\hline$K_{D_{u_{1}}}$ & $1 \cdot 10^{-2}$ \\
\hline$K_{P_{u_{3}}}$ & $-1 \cdot 10^{-4}$ \\
\hline$K_{I_{u_{3}}}$ & $-1 \cdot 10^{-6}$ \\
\hline$K_{D_{u_{3}}}$ & $-1 \cdot 10^{-6}$ \\
\hline$K_{P_{u_{4}}}$ & $-1 \cdot 10^{-2}$ \\
\hline$K_{I_{u_{4}}}$ & $-7.85 \cdot 10^{-4}$ \\
\hline$K_{D_{u_{4}}}$ & $-1 \cdot 10^{-2}$ \\
\hline$K_{P_{u_{5}}}$ & 1.5 \\
\hline$K_{I_{u_{5}}}$ & $3 \cdot 10^{-3}$ \\
\hline$K_{D_{u_{5}}}$ & 1.3 \\
\hline$K_{P_{u_{6}}}$ & 1.5 \\
\hline$K_{I_{u_{6}}}$ & $1.6 \cdot 10^{-3}$ \\
\hline$K_{D_{u_{6}}}$ & 1.5 \\
\hline &
\end{tabular}
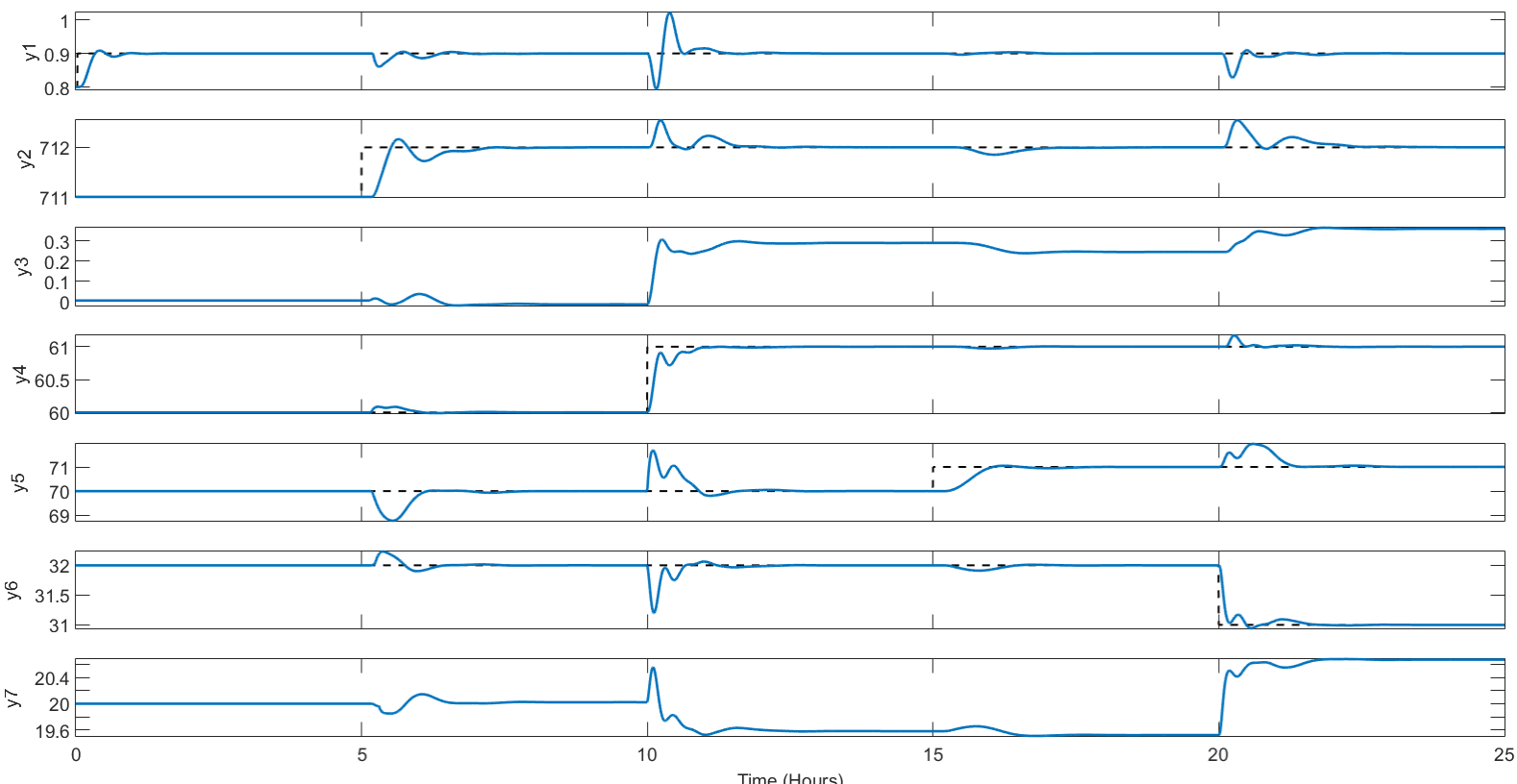

Figure 5.22 - PID Control for the FCC Benchmark. 


\subsubsection{Supervisory Control Layer Design}

The second task is to design the MPC that will generate setpoints for the PID controllers. This task begins with the identification of the process in closed loop, using the same procedure from the previous application, as described in (Garcia, et al., 2014). MISO models are identified for each output and combined into a MIMO model. The final model is compared with the process step response in Figure 5.23, with steps being applied to each PID setpoint and to $u_{2}$ at instants $0,5,10,15,20$ and 25, respectively.

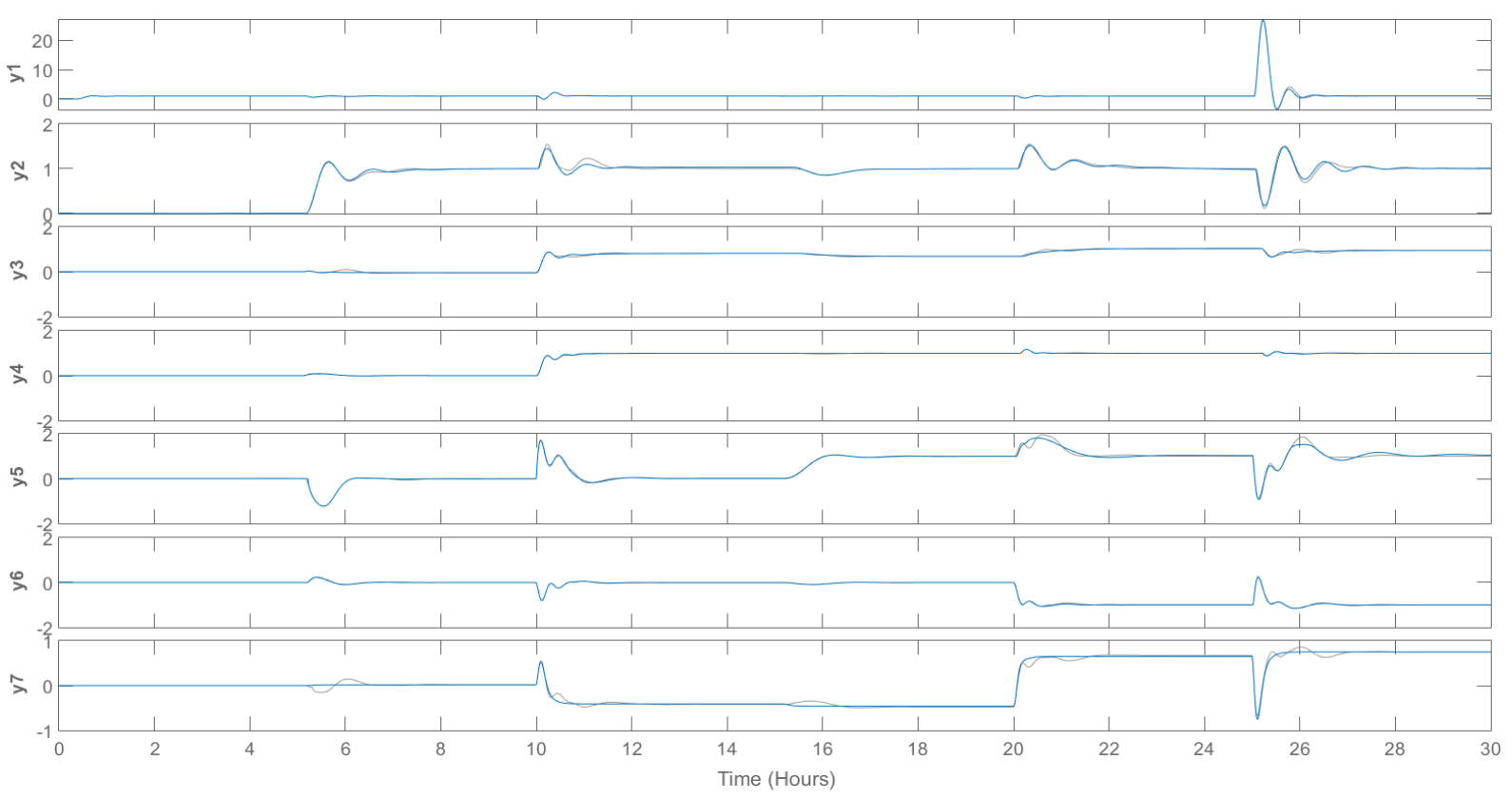

Figure 5.23 - Identified model for the FCC Plant in closed loop with PID controllers. Process (grey) and model (blue) responses.

The final step in the design of this layer is to tune the MPC controller. A sampling time of 1 minute is specified. For the other control parameters, the same tuning problem of the PID layer is applied and solved with the Optimal Control Tuning Technique from Section 3.7. The MPC controller generates setpoints for the five PID controllers and directly manipulates $u_{2}$, which was not associated to any variable in the first step and affects all process outputs. The results are shown in Table 5.19. 
Table 5.19 - Optimal MPC Tuning Parameters for the FCC Benchmark.

\begin{tabular}{l|c}
\hline Controller Parameter & Value \\
\hline Sampling Time $(\mathrm{s})$ & 60 \\
\hline Prediction Horizon & 60 \\
\hline Control Horizon & 10 \\
\hline$y_{1}$ Weight & 0.2 \\
\hline$y_{2}$ Weight & 0.1 \\
\hline$y_{3}$ Weight & 0 \\
\hline$y_{4}$ Weight & 0.1 \\
\hline$y_{5}$ Weight & 0.1 \\
\hline$y_{6}$ Weight & 0.1 \\
\hline$y_{7}$ Weight & 0 \\
\hline$S P_{1}$ Weight & 0 \\
\hline$S P_{2}$ Weight & 0 \\
\hline$S P_{4}$ Weight & 0 \\
\hline$S P_{5}$ Weight & 0 \\
\hline$S P_{6}$ Weight & 0 \\
\hline$u_{2}$ Weight & 0 \\
\hline$\Delta S P_{1}$ Weight & 1 \\
\hline$\Delta S P_{2}$ Weight & 1 \\
\hline$\Delta S P_{4}$ Weight & 1 \\
\hline$\Delta S P_{5}$ Weight & 1 \\
\hline$\Delta S P_{6}$ Weight & 1 \\
\hline$\Delta u_{2}$ Weight & 1 \\
\hline
\end{tabular}

In the final configuration, the FCC is controlled by a PID layer operating at 1 second sampling time and an MPC layer generating setpoints for the PID controllers every 1 minute.

The step responses of the process with the MPC layer are presented in Figure 5.24 , compared with the system with only the PID layer. 


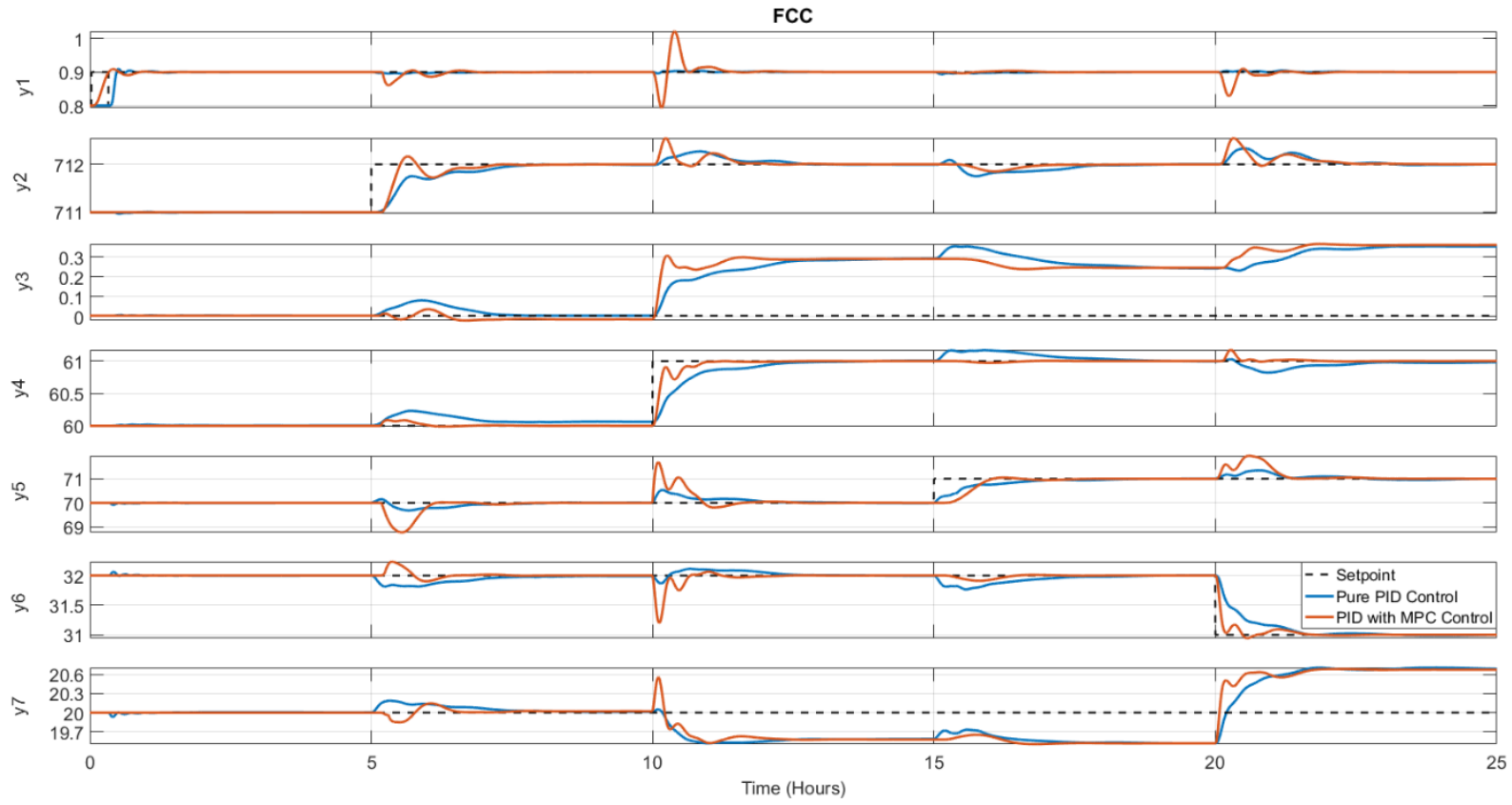

Figure 5.24 - Results for the FCC Benchmark.

For this system, the MPC was capable of enhancing the PID performance, being beneficial for the process. Performance indicators for both configurations are presented in Tables 5.20 and 5.21 and SSE performance indexes for all variables are shown in Table 5.22.

Table 5.20 - Performance Indicators for the FCC Benchmark [1].

\begin{tabular}{|c|c|c|c|}
\hline $\begin{array}{l}\text { Process } \\
\text { Variable }\end{array}$ & Performance Indicator & $\begin{array}{c}\text { Only PID } \\
\text { Control }\end{array}$ & $\begin{array}{c}\text { PID and MPC } \\
\text { Controllers }\end{array}$ \\
\hline \multirow{5}{*}{ 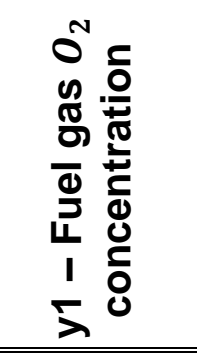 } & Settling Time (min) & 18.0 & 13.1 \\
\hline & Rise Time (min) & 16.5 & 7.7 \\
\hline & Overshoot (\%) & 8.9 & 10 \\
\hline & Highest Returning Time ( $\mathrm{min}$ ) & 133.3 & 83.3 \\
\hline & Maximum Deviation & 0.12 & 0.01 \\
\hline \multirow{5}{*}{ 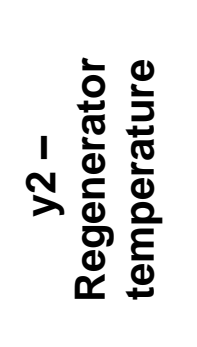 } & Settling Time (min) & 129.83 & 165.8 \\
\hline & Rise Time (min) & 28.3 & 114.3 \\
\hline & Overshoot (\%) & 20 & 0 \\
\hline & Highest Returning Time (min) & 141.7 & 183.3 \\
\hline & Maximum Deviation & 0.5 & 0.3 \\
\hline
\end{tabular}


Table 5.21 - Performance Indicators for the FCC Benchmark [2].

\begin{tabular}{|c|c|c|c|}
\hline $\begin{array}{l}\text { Process } \\
\text { Variable }\end{array}$ & Performance Indicator & $\begin{array}{c}\text { Only PID } \\
\text { Control }\end{array}$ & $\begin{array}{c}\text { PID and MPC } \\
\text { Controllers }\end{array}$ \\
\hline \multirow{5}{*}{$\begin{array}{l}\frac{9}{0} \\
\frac{0}{0} \\
\frac{0}{2} \\
\frac{1}{1} \\
\frac{m}{2}\end{array}$} & Settling Time (min) & - & - \\
\hline & Rise Time (min) & - & - \\
\hline & Overshoot (\%) & - & - \\
\hline & Highest Returning Time (min) & $\infty$ & $\infty$ \\
\hline & Maximum Deviation & 0.36 & 0.35 \\
\hline \multirow{5}{*}{ 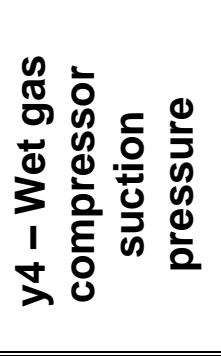 } & Settling Time (min) & 54.0 & 149.7 \\
\hline & Rise Time (min) & 12.7 & 105.3 \\
\hline & Overshoot (\%) & 0 & 0 \\
\hline & Highest Returning Time (min) & 56.7 & $\infty$ \\
\hline & Maximum Deviation & 0.17 & 0.23 \\
\hline \multirow{5}{*}{ 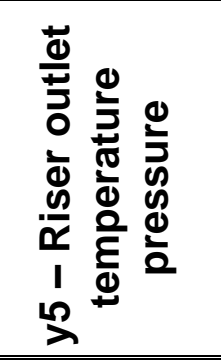 } & Settling Time (min) & 147.5 & 198.3 \\
\hline & Rise Time (min) & 55.5 & 102.5 \\
\hline & Overshoot (\%) & 5 & 0 \\
\hline & Highest Returning Time (min) & 168.3 & 248.8 \\
\hline & Maximum Deviation & 1.69 & 0.54 \\
\hline \multirow{5}{*}{ 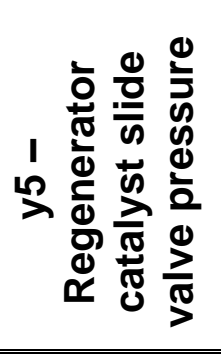 } & Settling Time (min) & 91.8 & 94.2 \\
\hline & Rise Time (min) & 8.5 & 77.3 \\
\hline & Overshoot (\%) & 6 & 0 \\
\hline & Highest Returning Time (min) & 107.7 & 165.0 \\
\hline & Maximum Deviation & 0.8 & 0.23 \\
\hline \multirow{5}{*}{ 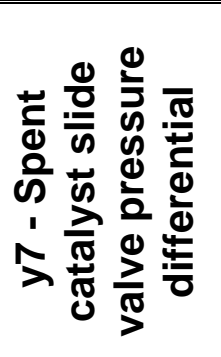 } & Settling Time (min) & - & - \\
\hline & Rise Time (min) & - & - \\
\hline & Overshoot (\%) & - & - \\
\hline & Highest Returning Time (min) & $\infty$ & $\infty$ \\
\hline & Maximum Deviation & 0.68 & 0.7 \\
\hline
\end{tabular}


Table 5.22 - SSE Performance Indexes for the FCC Benchmark.

\begin{tabular}{c|cc}
\hline Variable & SSE for PID Controllers & SSE for MPC with PID Controllers \\
\hline$y_{1}$ & 22.3 & 3.2 \\
\hline$y_{2}$ & 1896.8 & 2321.4 \\
\hline$y_{3}$ & 4637.2 & 11041.6 \\
\hline$y_{4}$ & 408.7 & 1420.9 \\
\hline$y_{5}$ & 8495.0 & 2118.9 \\
\hline$y_{6}$ & 641.1 & 1135.0 \\
\hline$y_{7}$ & 14087.3 & 31014.2 \\
\hline
\end{tabular}

\subsubsection{Results for the FCC Benchmark}

In this final step of the plantwide design procedure, the results are analyzed and the design decisions are reviewed and adjusted.

The control system designed for the FCC benchmark is composed of a regulatory control layer with five PID controllers operating at 1 second sampling time, controlling the main process variables, and a higher MPC layer operating at 1 minute sampling time, generating optimal trajectories for the PID setpoints and directly controlling one of the process inputs.

The designed control is capable of controlling the process according to the specifications. Comparing the results for the system with a higher MPC layer to those with only a PID layer, it can be noted that the MPC significantly reduces the deviation from setpoint for all variables, especially for $y_{1}$, the priority variable, which is kept much closer to its setpoint. This is achieved because the MPC is a native multivariable and predictive controller, acting on all variables simultaneously to optimize the overall system response over time, which highlights the advantage of the MPC for this process.

As in the previous application example, the plantwide control design technique guides the design and all decisions, including the final decision of keeping the initial dual layer architecture. 


\subsubsection{Concluding Remarks}

In this section, the techniques proposed in Chapter 4 were applied to five classical and to two non-square industrial benchmarks, a 5x7 HOF and a 6x7 FCC unit.

The results presented in Subsection 5.2.1 showed that I/O pairing and controller tuning are highly intertwined problems and should be solved simultaneously for better results. The proposed Optimal Multi-Objective Technique for Integrated Control Structure Selection and Tuning does so, and allows the use of multi-objective specifications to design optimal control systems, optimizing several independent or conflicting objectives.

The two control designs presented in Subsection 5.2.2 illustrate how the proposed techniques can be applied to generate optimal control systems for complex problems. Final results show that the MPC layer was necessary for only one of the tested benchmarks, the FCC. For the HOF benchmark, the PID controllers with optimal pairings and tunings were able to control the process, following operational constraints better than the system with the MPC controller. 


\section{Design of a Plantwide Control System to the Tennessee Eastman Challenge}

This chapter describes the application of the proposed Augmented Hierarchical Plantwide Control Design Technique and the proposed Optimal Multi-Objective Technique for Integrated Control Structure Selection and Controller Tuning to the Tennessee Eastman Challenge (Downs \& Vogel, 1993).

The control system design performed encompass the gathering of basic information about the process, the specification of the control system, several identifications of the process dynamics, the selection of variable pairings, the design of the controllers, controller tuning and performance evaluations.

The designed control system contains a regulatory layer with PID controllers and a supervisory layer with MPC controllers, following a standard architecture widely employed in the industry. The novelty resides in the design approach, with the proposed techniques providing new ways to obtain a good control system with a classical and well accepted architecture. The final control system was tested and validated in three different operating points.

Each step of the project and the results obtained are detailed next.

\subsection{The Tennessee Eastman Challenge Process}

The Tennessee Eastman Challenge was first published by (Downs \& Vogel, 1993). It consists of a model of an industrial chemical process for developing, studying, and evaluating process control technology, including plantwide control design techniques. The process has five major unit operations: the reactor, the product condenser, a vapor-liquid separator, a recycle compressor and a product stripper, as depicted in Figure 6.1 (Downs \& Vogel, 1993).

The process has 12 manipulated and 41 measured variables, from which 22 correspond to continuous process variables and 19 to sampled analytical variables. The lists of manipulated variables and continuous and sampled measured variables are presented in Tables 6.1, 6.2 and 6.3, respectively. 


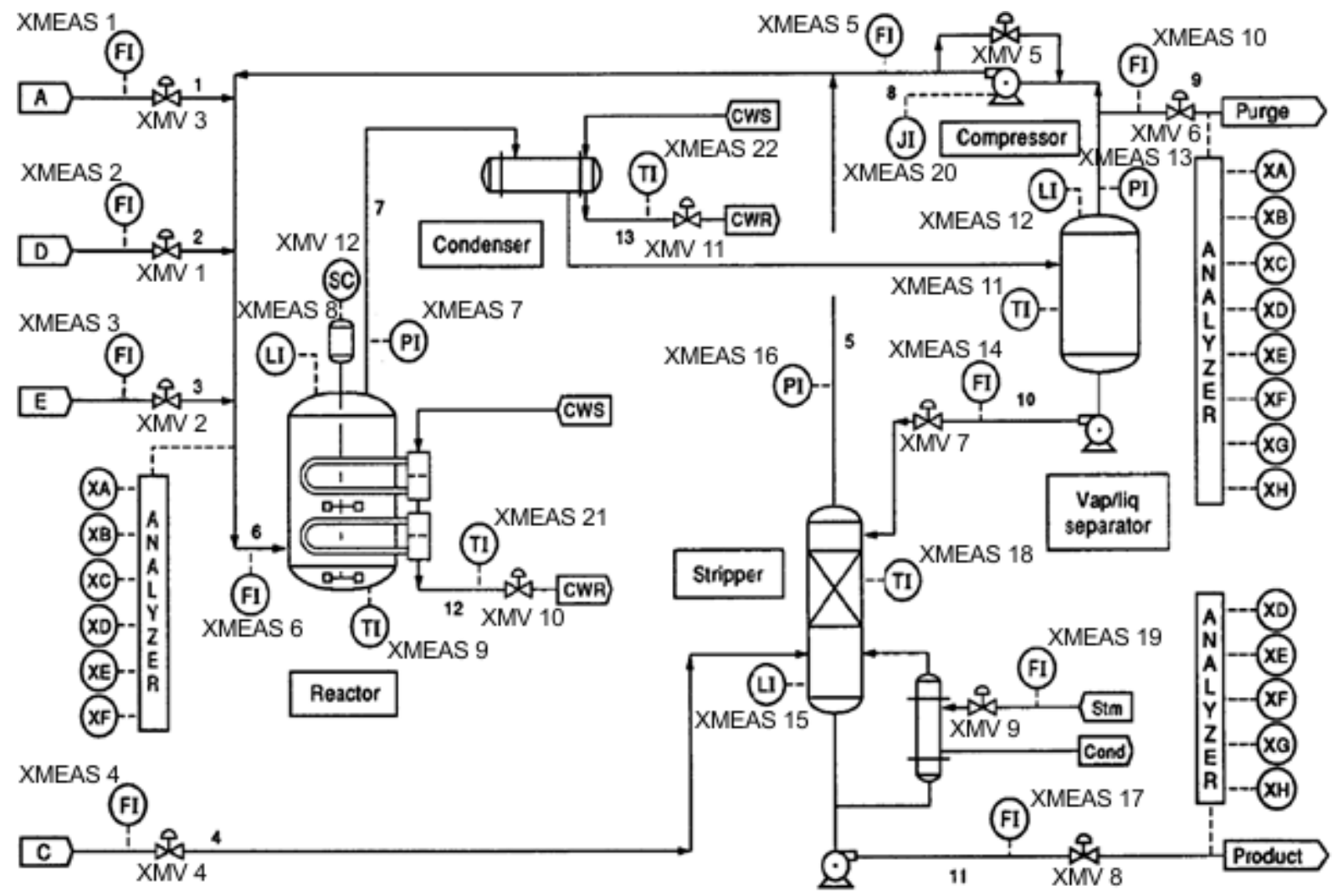

Figure 6.1 - The Tennessee Eastman Challenge Process.

Table 6.1 - Tennessee Eastman Process Manipulated Variables (Downs \& Vogel, 1993).

\begin{tabular}{l|c|c|c|c|c}
\hline \multicolumn{1}{c|}{ Variable Name } & $\begin{array}{c}\text { Variable } \\
\text { Number }\end{array}$ & $\begin{array}{c}\text { Nominal } \\
\text { Value (\%) }\end{array}$ & $\begin{array}{c}\text { Low } \\
\text { Limit }\end{array}$ & $\begin{array}{c}\text { High } \\
\text { Limit }\end{array}$ & Unit \\
\hline D feed flow (stream 2) & XMV 1 & 63.053 & 0 & 5111 & $\mathrm{~kg} / \mathrm{h}$ \\
\hline E feed flow (stream 3) & XMV 2 & 53.980 & 0 & 8354 & $\mathrm{~kg} / \mathrm{h}$ \\
\hline A feed flow (stream 1) & XMV 3 & 24.644 & 0 & 1.017 & $\mathrm{kscmh}$ \\
\hline A and C feed flow (stream 4) & XMV 4 & 61.302 & 0 & 15.25 & $\mathrm{kscmh}$ \\
\hline Compressor recycle valve & XMV 5 & 22.210 & 0 & 100 & $\%$ \\
\hline Purge valve (stream 9) & XMV 6 & 40.064 & 0 & 100 & $\%$ \\
\hline $\begin{array}{l}\text { Separator pot liquid flow (stream } \\
\text { 10) }\end{array}$ & XMV 7 & 38.100 & 0 & 65.71 & $\mathrm{~m} 3 / \mathrm{h}$ \\
\hline $\begin{array}{l}\text { Stripper liquid product flow (stream } \\
\text { 11) }\end{array}$ & XMV 8 & 46.534 & 0 & 49.10 & $\mathrm{~m} 3 / \mathrm{h}$ \\
\hline Stripper steam valve & XMV 9 & 47.446 & 0 & 100 & $\%$ \\
\hline Reactor cooling water flow & XMV 10 & 41.106 & 0 & 227.1 & $\mathrm{~m} 3 / \mathrm{h}$ \\
\hline Condenser cooling water flow & XMV 11 & 18.114 & 0 & 272.6 & $\mathrm{~m} 3 / \mathrm{h}$ \\
\hline Agitator speed & XMV 12 & 50.000 & 150 & 250 & $\mathrm{rpm}$ \\
\hline
\end{tabular}


Table 6.2 - Tennessee Eastman Process Continuous Measured Variables (Downs \& Vogel, 1993).

\begin{tabular}{l|c|c|c}
\hline \multicolumn{1}{c|}{ Variable Name } & Variable & Nominal Value & Unit \\
& Number & $(\%)$ & \\
\hline A feed (stream 1) & XMEAS 1 & 0.25052 & $\mathrm{kscmh}$ \\
\hline D feed (stream 2) & XMEAS 2 & 3664.0 & $\mathrm{~kg} / \mathrm{h}$ \\
\hline E feed (stream 3) & XMEAS 3 & 4509.3 & $\mathrm{~kg} / \mathrm{h}$ \\
\hline A and C feed (stream 4) & XMEAS 4 & 9.3477 & $\mathrm{kscmh}$ \\
\hline Recycle flow (stream 8) & XMEAS 5 & 26.902 & $\mathrm{kscmh}$ \\
\hline Reactor feed rate (stream 6) & XMEAS 6 & 42.339 & $\mathrm{kscmh}$ \\
\hline Reactor pressure & XMEAS 7 & 2705.0 & $\mathrm{kPa}$ \\
\hline Reactor level & XMEAS 8 & 75.0 & $\%$ \\
\hline Reactor temperature & XMEAS 9 & 120.40 & ${ }^{\circ} \mathrm{C}$ \\
\hline Purge rate (stream 9) & XMEAS 10 & 0.33712 & $\mathrm{kscmh}$ \\
\hline Product separator temperature & XMEAS 11 & 80.109 & ${ }^{\circ} \mathrm{C}$ \\
\hline Product separator level & XMEAS 12 & 50.0 & $\%$ \\
\hline Product separator pressure & XMEAS 13 & 2633.7 & $\mathrm{kPa}$ \\
\hline Product separator underflow (stream 10) & XMEAS 14 & 25.160 & $\mathrm{~m} 3 / \mathrm{h}$ \\
\hline Stripper level & XMEAS 15 & 50.0 & $\%$ \\
\hline Stripper pressure & XMEAS 16 & 3102.2 & $\mathrm{kPa}$ \\
\hline Stripper underflow (stream 11) & XMEAS 17 & 22.949 & $\mathrm{~m} 3 / \mathrm{h}$ \\
\hline Stripper temperature & XMEAS 18 & 65.731 & ${ }^{\circ} \mathrm{C}$ \\
\hline Stripper steam flow & XMEAS 19 & 230.31 & $\mathrm{~kg} / \mathrm{h}$ \\
\hline Compressor work & XMEAS 20 & 341.43 & $\mathrm{~kW}$ \\
\hline Reactor cooling water outlet temperature & XMEAS 21 & 94.599 & ${ }^{\circ} \mathrm{C}$ \\
\hline Separator cooling water outlet temperature & XMEAS 22 & 77.297 & ${ }^{\circ} \mathrm{C}$ \\
\hline & & & \\
\hline
\end{tabular}

All the design steps and identification experiments were performed considering the nominal operation conditions and default disturbance scenarios. At the end of the project, alternative operating points were used to test and to validate the plantwide control system, after performing minor adjustments. 
Table 6.3 - Tennessee Eastman Process Sampled Analytical Variables (Downs \& Vogel, 1993).

\begin{tabular}{l|c|c|c}
\hline \multicolumn{1}{c|}{ Variable Name } & Variable Number & Nominal Value (\%) & Unit \\
\hline $\mathrm{A}$ in the reactor feed analysis & XMEAS 23 & 32.188 & $\mathrm{~mol} \%$ \\
\hline $\mathrm{B}$ in the reactor feed analysis & XMEAS 24 & 8.8933 & $\mathrm{~mol} \%$ \\
\hline $\mathrm{C}$ in the reactor feed analysis & XMEAS 25 & 26.383 & $\mathrm{~mol} \%$ \\
\hline $\mathrm{D}$ in the reactor feed analysis & XMEAS 26 & 6.8820 & $\mathrm{~mol} \%$ \\
\hline $\mathrm{E}$ in the reactor feed analysis & XMEAS 27 & 18.776 & $\mathrm{~mol} \%$ \\
\hline $\mathrm{F}$ in the reactor feed analysis & XMEAS 28 & 1.6567 & $\mathrm{~mol} \%$ \\
\hline $\mathrm{A}$ in the purge gas analysis & XMEAS 29 & 32.958 & $\mathrm{~mol} \%$ \\
\hline $\mathrm{B}$ in the purge gas analysis & XMEAS 30 & 13.823 & $\mathrm{~mol} \%$ \\
\hline $\mathrm{C}$ in the purge gas analysis & XMEAS 31 & 23.978 & $\mathrm{~mol} \%$ \\
\hline $\mathrm{D}$ in the purge gas analysis & XMEAS 32 & 1.2565 & $\mathrm{~mol} \%$ \\
\hline $\mathrm{E}$ in the purge gas analysis & XMEAS 33 & 18.579 & $\mathrm{~mol} \%$ \\
\hline $\mathrm{F}$ in the purge gas analysis & XMEAS 34 & 2.2633 & $\mathrm{~mol} \%$ \\
\hline $\mathrm{G}$ in the purge gas analysis & XMEAS 35 & 4.8436 & $\mathrm{~mol} \%$ \\
\hline $\mathrm{H}$ in the purge gas analysis & XMEAS 36 & 2.2986 & $\mathrm{~mol} \%$ \\
\hline $\mathrm{D}$ in the product analysis & XMEAS 37 & 0.01787 & $\mathrm{~mol} \%$ \\
\hline $\mathrm{E}$ in the product analysis & XMEAS 38 & 0.83570 & $\mathrm{~mol} \%$ \\
\hline $\mathrm{F}$ in the product analysis & XMEAS 39 & 0.09858 & $\mathrm{~mol} \%$ \\
\hline $\mathrm{G}$ in the product analysis & XMEAS 40 & 53.724 & $\mathrm{~mol} \%$ \\
\hline $\mathrm{H}$ in the product analysis & XMEAS 41 & 43.828 & $\mathrm{~mol} \%$ \\
\hline
\end{tabular}

This benchmark can be used in several manners and for many different goals. For this study, the following objectives, based in (Downs \& Vogel, 1993), were chosen to be achieved:

1. Maintain process variables at desired values;

2. Keep process operating conditions within equipment constraints;

3. Minimize variability during disturbances;

4. Minimize changes in variables which affect other processes (gas feeds);

5. Recover quickly and smoothly from disturbances and setpoint changes.

The operational constraints are presented in Table 6.4. The high and low shutdown limits are used to shut down the process in instability scenarios. These limits are hardcoded in the plant simulator, which stops if they are reached. 
Table 6.4 - Tennessee Eastman Process Operating Constraints (Downs \& Vogel, 1993).

\begin{tabular}{l|c|c|c|c}
\hline \multirow{2}{*}{ Process Variable } & \multicolumn{2}{|c|}{ Normal Operating Limits } & \multicolumn{2}{c}{ Shutdown Limits } \\
\cline { 2 - 5 } & Low Limit & High Limit & Low Limit & High Limit \\
\hline Reactor Pressure & - & $2895 \mathrm{kPa}$ & - & $3000 \mathrm{kPa}$ \\
\hline Reactor Level & $50 \%$ & $100 \%$ & $2.0 \mathrm{~m}^{3}$ & $24.0 \mathrm{~m}^{3}$ \\
\hline Reactor Temperature & - & $150 \% \mathrm{C}$ & - & $175^{\circ} \mathrm{C}$ \\
\hline Reactor Separator Level & $30 \%$ & $100 \%$ & $1.0 \mathrm{~m}^{3}$ & $12.0 \mathrm{~m}^{3}$ \\
\hline Stripper Base Level & $30 \%$ & $100 \%$ & $1.0 \mathrm{~m}^{3}$ & $8.0 \mathrm{~m}^{3}$ \\
\hline
\end{tabular}

This benchmark model also includes an operation cost measurement, which allows a quantitative evaluation of control performance. The operation cost is determined by the loss of raw materials in the purge gas, the product stream and by side reactions, compressor work and steam used in the stripping column (Downs \& Vogel, 1993). A simplified form of the total cost is presented in Equation (6.1).

$$
\begin{gathered}
\text { Total Costs }=(\text { Purge Costs }) \cdot(\text { Purge Rate })+ \\
(\text { Product Stream Costs }) \cdot(\text { Product Rate })+ \\
(\text { Compressor Costs }) \cdot(\text { Compressor Work })+ \\
(\text { Steam Costs }) \cdot(\text { Steam Rate })
\end{gathered}
$$

\subsection{Implementation of the Tennessee Eastman Model}

For this project, the revised implementation of the Tennessee Eastman Challenge process model described in (Bathelt, et al., 2015) was employed. This revised model improves the model accuracy and stability and includes new output variables, with 73 outputs in total. As stated by the authors in (Bathelt, et al., 2015), although being an old model, the Tennessee Eastman Challenge remains an important benchmark for many disciplines in control theory, including Plantwide Control and System Identification. The main advantage of this benchmark resides in the fact that it was not artificially conceived, but carefully modeled based on a real plant, presenting a complex system with several dynamic relations.

The final version of the control system designed in this work was developed in MATLAB $^{\circledR}$ R2016b (9.1.0.441655). The model code (temexd_mod.c) provided by (Bathelt, et al., 2015) was embedded in a Simulink model, as depicted in Figure 6.2. 


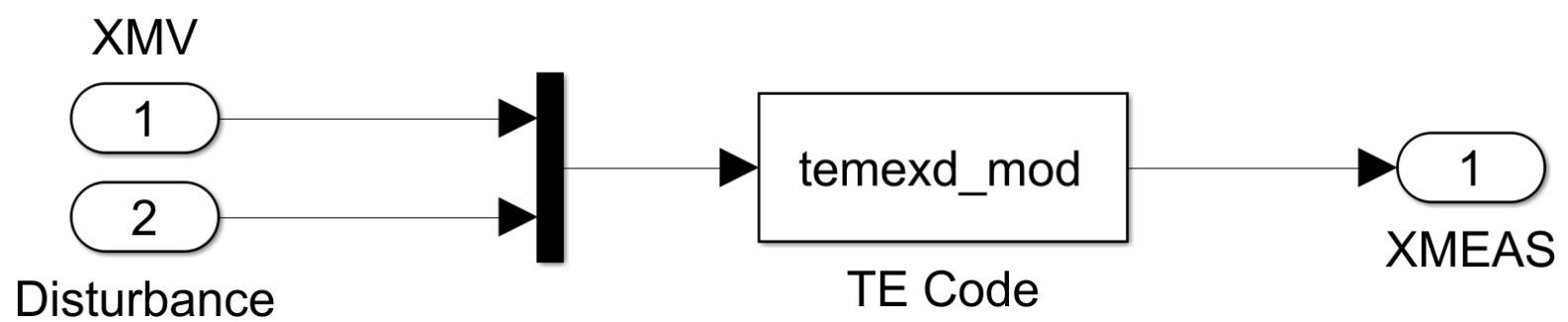

Figure 6.2 - Tennessee Eastman Challenge Model Implementation.

The model function receives as input a vector with the manipulated variables and the disturbance configuration, and returns a vector with the process outputs. It is possible to specify the model initial conditions, seed for the random generator and define additional features of the revised model.

For all design steps and identification experiments, default initial conditions and disturbance profiles were employed. These values were only changed for the final tests in different operation modes, as explicitly described in the final steps of this description.

For the simulation setup, the Dormand-Prince (ode45) algorithm with variablestep and relative tolerance of $10^{-3}$ was used as solver.

\subsection{The Augmented Hierarchical Plantwide Control Design Technique}

The control system design for the Tennessee Eastman Challenge was performed following the Augmented Hierarchical Plantwide Control Design Technique proposed in Section 4.2. The execution of each procedure step is detailed ahead.

The procedure starts with the pure process in open-loop (unstable) and design a plantwide control system for stabilization, operation constraints satisfaction, setpoint tracking, disturbance rejection and economical optimization. The main characteristics of this procedure are the integration of system identification and optimal control tuning to the plantwide control design and support to an optimal multi-objective specification. 


\subsection{The Optimal Multi-Objective Technique for Integrated Control Structure Selection and Controller Tuning}

The tuning of all controllers and final refinement of input-output pairings were performed employing the Optimal Multi-Objective Technique for Integrated Control Structure Selection and Controller Tuning proposed in Section 4.3.

\subsection{The System Identification Platform Used}

To emulate a control design procedure applied to a real plant, the Tennessee Eastman Challenge process was considered as a black-box, i.e., no explicit model relations (from the model code) were taken into consideration during the control design, only basic information provided in the process description from (Downs \& Vogel, 1993).

All dynamic information of the process is obtained using System Identification. All identifications were performed using the Computer-Aided System Identification (CASI) toolbox, developed in a research project by the author and his supervisor.

The identifications performed (and the CASI toolbox) encompass the experiment design, data collection, data pre-processing, selection of order and delay, model estimation, model validation and model selection.

Identified models were employed in the design and tuning of all the controllers.

\subsection{Design of the Control System}

The design of the control plantwide system follows the steps of the proposed Augmented Hierarchical Plantwide Control Design Technique. The first set of steps consists of top-down procedures, in which general decisions are taken and the control is specified. The second set of steps are design steps, in which each controller is designed and tuned in a bottom-up procedure.

The specification (top-down) procedure starts with the gathering of information about the process. Next, the control specifications are defined based on the 
information gathered and on additional considerations. Finally, each control layer is defined and its design requisites are specified.

The design (bottom-up) steps consist of the proper design of each controller, including the identification of process dynamics and optimal controller tuning. It includes the design of all control layers and final adjustments to the control system.

All steps of the procedure are presented next. Section numeration follows the step numeration used in the procedure description in Section 4.2 for better readability.

\subsubsection{I-Specification (Top-Down) Steps}

The first set of steps of the plantwide control design procedure deals with the determination of all specifications for the control system. Each step is presented next.

\section{Information Gathering}

The first step of the procedure is the gathering of information about the process. This includes the description of the process, plant units, process variables and control objectives. The most important information acquired about the Tennessee Eastman process was presented in the previous sections.

The process was depicted in Figure 6.1. It consists of five main units: reactor, product condenser, vapor-liquid separator, recycle compressor and product stripper.

The process has 12 manipulated variables, 28 (unmeasured) disturbance variables, 22 continuous measured variables, 19 sampled analytical variables and 32 auxiliary output variables (secondary sensors and some internal states not normally available, included on the revised form of the model). The process variables and its main properties were presented in Tables 6-1, 6-2 and 6-3.

For this work, based on approaches presented in many publications, the continuous process variables (XMEAS $1-22$ ) and the 12 manipulated variables were used to build the control system. The disturbances were kept active with default configurations in all experiments and tests. 


\section{General Control Specifications}

The control specifications (performance and robustness requirements) chosen to be pursued are:

1. Maintain process variables at desired values;

2. Keep process operating conditions within equipment constraints;

3. Minimize variability during disturbances;

4. Minimize changes in variables which affect other processes (gas feeds);

5. Recover quickly and smoothly from disturbances and setpoint changes.

The operating constraints of the process were presented in Table 6.4.

For the economic optimization, the operation cost, represented by Equation (6.1), is to be minimized by the control system. An analysis of the cost function shows that the following process variables directly affect the operation cost: XMEAS 8, XMEAS 10, XMEAS 19 and XMEAS 20.

The next step is to determine the candidate measurements $y$ and candidate manipulated variables $u$. Since the process has more outputs than manipulated variables, all 12 manipulated variables (MVs) are eligible to be used by the controllers.

A first inspection of the process dynamics in open-loop operation reveals that the following variables are unstable and need to be controlled:

1. XMEAS 7: Reactor pressure;

2. XMEAS 8: Reactor level;

3. XMEAS 9: Reactor temperature;

4. XMEAS 12: Product separator level;

5. XMEAS 15: Stripper base level.

For steady-state operation conditions, the setpoints specified by (Bathelt, et al., 2015) for all variables are employed. For the remainder of the project, the setpoints in nominal operating point are used.

\section{Plantwide Control Layer}

The first step to define the plantwide control layer is to choose the main plantwide control objective. For this process, this objective is well defined as the 
minimization of the objective cost, described by Equation (6.1), subject to the satisfaction of operating constraints.

An MPC controller is chosen to optimize the operation cost and to keep the process under operating constraints, by determining the setpoints to the lower control layer.

\section{Supervisory Control Layer}

The supervisory control layer needs to control and optimize each of the process units (reactor, product condenser, vapor-liquid separator, recycle compressor and product stripper).

To obtain dynamic models for each unit, identification experiments with the regulatory layer in closed-loop were performed at the design step of these controllers.

For this layer, the MPC control structure is chosen. It is necessary to be later determined if an independent MPC will be built for each unit, or if a single MPC for all units is feasible. If this latter option demonstrates to be the best, it will also be considered if this MPC will be able to perform the plantwide optimization of the process (as shown later, this last option was chosen for the control system).

Based on the information gathered about the process, a sampling time of 1 minute is initially chosen for this control layer (this decision was kept in the final design).

\section{Regulatory Control Layer}

The final top-down step is the specification of the regulatory control level. The controllers on this level will directly manipulate the process valves and receive setpoints from the upper layers.

The first objective for this control layer is to stabilize the following unstable process variables:

1. XMEAS 7: Reactor pressure;

2. XMEAS 8: Reactor level;

3. XMEAS 9: Reactor temperature;

4. XMEAS 12: Product separator level;

5. XMEAS 15: Stripper base level. 
The next important objective of the regulatory layer is to minimize the variability of the gas feed, which are:

1. XMEAS 1: A feed flow;

2. XMEAS 2: D feed flow;

3. XMEAS 3: $E$ feed flow;

4. XMEAS 4: A and $C$ feed flow.

After these objectives are achieved, the remaining unused manipulated variables will be screened to be used to control other measured variables and to optimize the operation economics.

PID controllers with sampling time of 1 second are initially chosen for the regulatory layer (this decision was kept in the final control design).

\subsubsection{II - Design (Bottom-Up) Steps}

The second set of steps of the plantwide control design procedure deals with the proper design of the controllers. These steps hold the main novelties of the proposed procedure, which are the integration of System Identification and optimal controller tuning in the plantwide design technique. This allows a better knowledge of the process and the final results to be considered during the design steps, improving the key design decisions and creating a final implemented control system that behaves close to the designed one. Each design step is detailed next.

\section{Regulatory Control Layer}

The first step of the bottom-up design is the creation of the regulatory control layer. The decisions taken on the specification steps are revised and the controllers are properly built.

The first part of this step is to obtain a dynamic model for the variables to be controlled at the regulatory level. Since the process is unstable, the implementation of controllers to stabilize it is necessary, prior to performing the identification experiments. After the process is stabilized, the identification experiments are carried out to obtain a model of the process. The identification results are then used to determine the optimal pairings and the optimal control tuning for the controlled variables. 
The design procedure and results are detailed next.

\section{Stabilization of the Process}

The design of the stabilizing control starts with simple step experiments to determine which variables most affect the five unstable process variables.

A step of low amplitude is applied to each manipulated variable and the responses of the process output variables are collected for a short time (while the process is under safe conditions).

The data collected is then used to determine a matrix of effects between manipulated and unstable measured variables. The resulting $1 / O$ effect matrix is presented in Table 6.5. In it, and in all the next I/O effect matrices that follows, ' + ' indicates an influence with positive gain, '-' indicates a negative gain, ' 0 ' indicates the absence of influence and '*' indicates an uncertainty in the determined influence.

Table 6.5 - I/O effect matrix for unstable variables in open loop.

\begin{tabular}{|c|c|c|c|c|c|}
\hline & \multicolumn{5}{|c|}{ Unstable Measured Variables } \\
\hline & $\begin{array}{c}\text { XMEAS } 7 \\
\text { Reactor } \\
\text { Pressure }\end{array}$ & $\begin{array}{c}\text { XMEAS } 8 \\
\text { Reactor } \\
\text { Level }\end{array}$ & $\begin{array}{c}\text { XMEAS } 9 \\
\text { Reactor } \\
\text { Temperature }\end{array}$ & $\begin{array}{c}\text { XMEAS } 12 \\
\text { Separator } \\
\text { Level }\end{array}$ & $\begin{array}{l}\text { XMEAS } 15 \\
\text { Striper Level }\end{array}$ \\
\hline XMV 1 & - & + & + & - & 0 \\
\hline XMV 2 & - & + & + & + & 0 \\
\hline XMV 3 & + & - & + & + & 0 \\
\hline XMV 4 & + & - & + & + & - \\
\hline XMV 5 & * & * & + & - & 0 \\
\hline XMV 6 & - & - & + & + & - \\
\hline XMV 7 & + & - & 0 & - & + \\
\hline XMV 8 & 0 & 0 & 0 & 0 & - \\
\hline XMV 9 & 0 & 0 & + & 0 & 0 \\
\hline XMV 10 & + & - & - & - & 0 \\
\hline XMV 11 & - & - & - & + & - \\
\hline XMV 12 & 0 & 0 & - & 0 & 0 \\
\hline
\end{tabular}

Next, dynamic models are identified for the unstable variables and the manipulated variables which affect them, using the process responses. 
The Optimal Multi-Objective Technique for Integrated Control Structure Selection and Controller Tuning is then applied to the model, to determine the stabilizing controllers. The following I/O pairs are encountered:

D XMEAS 7 (reactor pressure) $\times$ XMV 6 (purge valve);

$>\quad$ XMEAS 8 (reactor level) $\times$ XMV 11 (condenser cooling water flow);

$>\quad$ XMEAS 9 (reactor temperature) $\times$ XMV 10 (reactor cooling water flow);

$>\quad$ XMEAS 12 (product separator level) $\times$ XMV 7 (separator pot liquid flow);

$>\quad$ XMEAS 15 (stripper level) $\times$ XMV 8 (stripper liquid product flow).

Controllers are implemented to control the unstable variables with the manipulated variables that affect them most strongly. Figure 6.3 shows the Simulink diagram of the plant with the implemented stabilizing controllers.
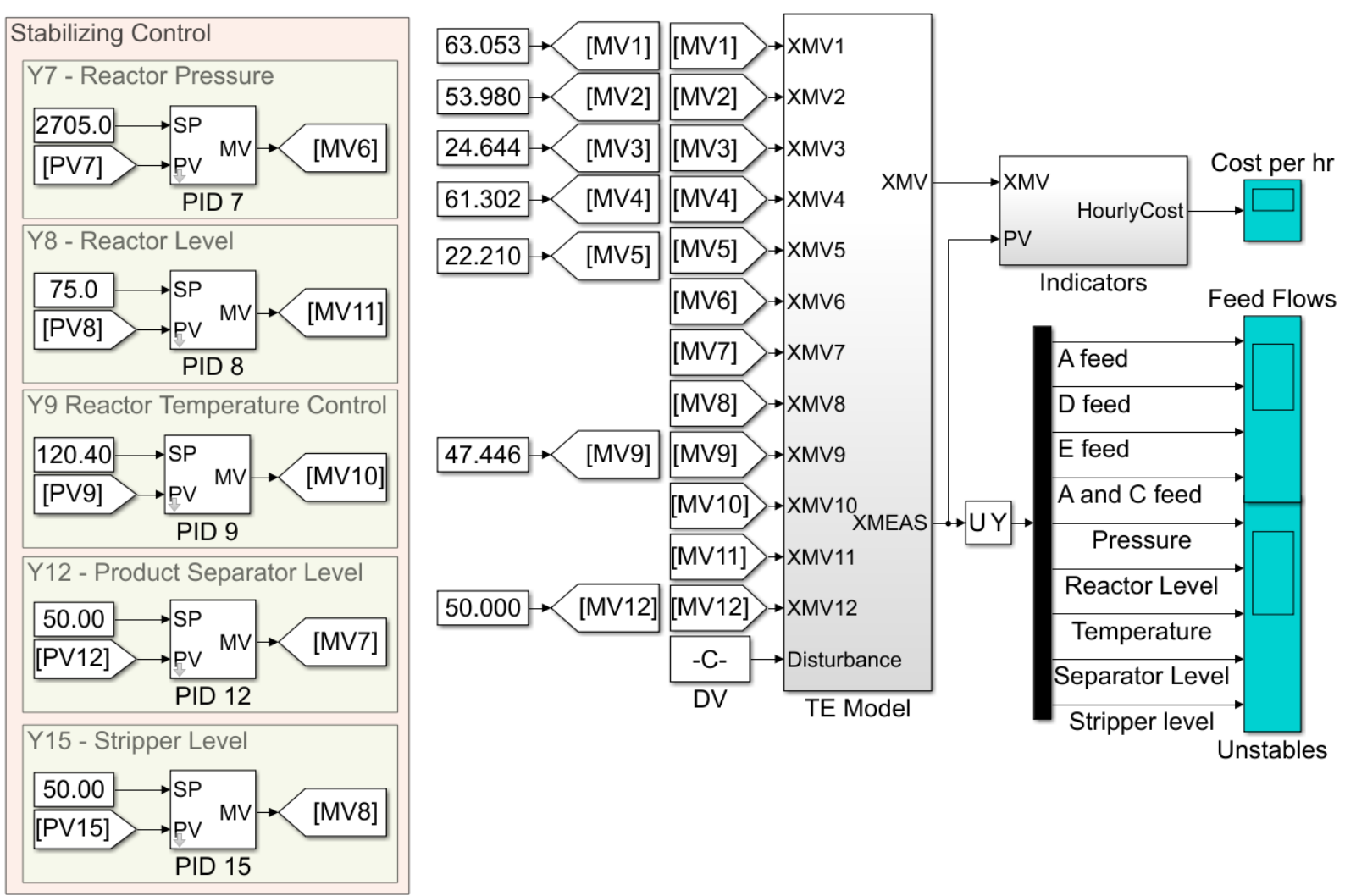

Figure 6.3 - Tennessee Eastman with stabilizing control.

\section{Optimal Pairings for the Unstable Variables}

After a first stabilizing control is implemented, it is possible to perform better identification experiments on the process. 
For these new tests, step sequences are applied to all manipulated variables that affect the unstable outputs and also to the setpoints of the four stabilizing controllers. The responses of all the process outputs are collected and analyzed.

First, a new $\mathrm{I} / \mathrm{O}$ effect matrix is created for all measured variables based on the resulting data. The resulting $\mathrm{I} / \mathrm{O}$ effect matrix is presented in Table 6.6, in which ' $\mathrm{X}$ ' indicates relations for which the influences were not determined (either because the relation was not a candidate for pairing at this stage or because the collected data was not representative enough).

Table 6.6 - I/O effect matrix for the stabilized plant.

\begin{tabular}{|c|c|c|c|c|c|c|c|c|c|c|c|c|c|}
\hline & \multicolumn{12}{|c|}{ Manipulated Variables (XMV) } \\
\hline & & 1 & 2 & 3 & 4 & 5 & 6 & 7 & 8 & 9 & 10 & 11 & 12 \\
\hline \multirow{22}{*}{ 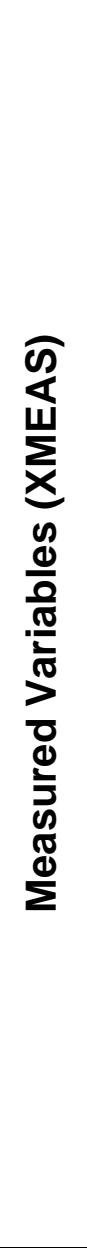 } & 1 & 0 & 0 & + & 0 & 0 & 0 & 0 & 0 & 0 & 0 & 0 & 0 \\
\hline & 2 & + & 0 & 0 & 0 & 0 & 0 & 0 & 0 & 0 & 0 & 0 & 0 \\
\hline & 3 & 0 & + & 0 & 0 & 0 & 0 & 0 & 0 & 0 & 0 & 0 & 0 \\
\hline & 4 & 0 & 0 & 0 & + & 0 & 0 & 0 & 0 & 0 & 0 & 0 & 0 \\
\hline & 5 & 0 & 0 & 0 & + & - & $\mathrm{X}$ & $\mathrm{X}$ & $\mathrm{X}$ & 0 & $\mathrm{X}$ & $\mathrm{X}$ & $++^{*}$ \\
\hline & 6 & 0 & 0 & 0 & + & - & $\mathrm{X}$ & $X$ & $\mathrm{X}$ & 0 & $\mathrm{X}$ & $\mathrm{X}$ & + \\
\hline & 7 & + & + & + & + & + & + & + & 0 & 0 & + & - & $--^{*}$ \\
\hline & 8 & + & + & 0 & + & + & - & - & 0 & 0 & - & - & 0 \\
\hline & 9 & + & 0 & 0 & + & + & + & 0 & 0 & 0 & - & - & - \\
\hline & 10 & - & 0 & + & + & - & + & $X$ & $X$ & 0 & $X$ & $X$ & $-^{*}$ \\
\hline & 11 & - & - & 0 & - & 0 & $X$ & $X$ & $X$ & 0 & $X$ & $X$ & $--^{*}$ \\
\hline & 12 & - & + & 0 & 0 & 0 & + & - & 0 & 0 & - & + & 0 \\
\hline & 13 & - & + & + & + & + & $X$ & $\mathrm{X}$ & $X$ & 0 & $X$ & $X$ & $-^{*}$ \\
\hline & 14 & + & + & 0 & 0 & 0 & $X$ & $X$ & $X$ & 0 & $X$ & $X$ & 0 \\
\hline & 15 & + & + & 0 & - & 0 & - & + & - & 0 & 0 & - & 0 \\
\hline & 16 & - & + & + & + & - & $X$ & $X$ & $X$ & 0 & $X$ & $X$ & $-^{*}$ \\
\hline & 17 & + & + & 0 & + & - & 0 & - & + & 0 & 0 & - & 0 \\
\hline & 18 & - & + & 0 & - & - & $X$ & $X$ & $X$ & + & $X$ & $X$ & 0 \\
\hline & 19 & + & + & 0 & + & + & $X$ & $X$ & $X$ & + & $X$ & $X$ & 0 \\
\hline & 20 & + & + & 0 & + & 0 & $X$ & $X$ & $X$ & 0 & $X$ & $X$ & 0 \\
\hline & 21 & - & $-^{*}$ & 0 & - & 0 & $X$ & $X$ & $X$ & 0 & $X$ & $X$ & + \\
\hline & 22 & - & - & 0 & + & - & $X$ & $X$ & $X$ & 0 & $X$ & $X$ & 0 \\
\hline
\end{tabular}

System Identification is then applied to adjust models to describe the relationship between the measured and the manipulated variables. At this stage, the manipulated variables regulated by the PID controllers are also used in the 
identification, and not the controllers' setpoints. The identification procedure adopted is illustrated in Appendix A. This results in a precise model of the process in open loop.

Next, the Optimal Multi-Objective Technique for Integrated Control Structure Selection and Controller Tuning is applied again, to determine the best regulatory controllers for the unstable variables. As stated in the Augmented Plantwide Control Design Procedure, unlike in most approaches, the stabilizing controllers are not considered final and the unstable variables are again subject to the pairing procedure with all other variables.

This new pairing problem includes the manipulated variables which affect the unstable outputs and all candidate controlled variables affected by such manipulated variables (as determined by the $\mathrm{I} / \mathrm{O}$ effect matrix). This corresponds to the following candidate variables:

> Candidate manipulated variables: XMV 1, XMV 2, XMV 3, XMV 4, XMV 5, XMV 6, XMV 7, XMV 8, XMV 10 and XMV 11.

$>\quad$ Candidate measured variables: XMEAS 1, XMEAS 2, XMEAS 3, XMEAS 4, XMEAS 5, XMEAS 6, XMEAS 7, XMEAS 8, XMEAS 9, XMEAS 10, XMEAS 11, XMEAS 12, XMEAS 13, XMEAS 14, XMEAS 15, XMEAS 16, XMEAS 17, XMEAS 18, XMEAS 19, XMEAS 20, XMEAS 21, XMEAS 22.

The problem solution results in the following I/O pairings:

$>\quad$ XMEAS 7 (reactor pressure) $\times$ XMV 6 (purge valve);

$>\quad$ XMEAS 8 (reactor level) $\times$ XMV 11 (condenser cooling water flow);

$>\quad$ XMEAS 9 (reactor temperature) $\times$ XMV 10 (reactor cooling water flow);

$>\quad$ XMEAS 12 (product separator level) $\times$ XMV 7 (separator liquid product flow);

$>\quad$ XMEAS 15 (stripper level) $\times$ XMV 8 (stripper liquid product flow).

The defined PID controllers are then implemented with the optimal tuning provided by the algorithm and tested. The resulting configuration is able to keep the process stable and the controlled variables at their setpoints.

\section{Design of the Complete Regulatory Control Layer}

With the final PID controllers for the unstable variables defined, a new set of experiments can be performed with the stable process. 
A new set of step sequences is applied to the remaining manipulated variables (not previously excited) and to the setpoints of the implemented controllers.

The collected data is used to update the $\mathrm{I} / \mathrm{O}$ effect matrix and to identify models to the remaining $\mathrm{I} / \mathrm{O}$ relationship. The resulting $\mathrm{I} / \mathrm{O}$ effect matrix is presented in Table 6.7 .

Table 6.7 - I/O effect matrix for the stabilized plant.

\begin{tabular}{|c|c|c|c|c|c|c|c|c|c|c|c|c|c|c|c|c|c|c|}
\hline & \multicolumn{12}{|c|}{ Manipulated Variables (XMV) } & \multicolumn{5}{|c|}{ Setpoints (SP) } \\
\hline & & 1 & 2 & 3 & 4 & 5 & 6 & 7 & 8 & 9 & 10 & 11 & 12 & 7 & 8 & 9 & 12 & 15 \\
\hline \multirow{22}{*}{ 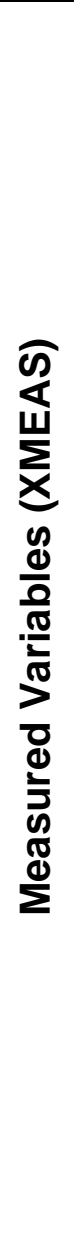 } & 1 & 0 & 0 & + & 0 & 0 & $X$ & $X$ & $X$ & 0 & $X$ & $X$ & 0 & 0 & 0 & 0 & 0 & 0 \\
\hline & 2 & + & 0 & 0 & 0 & 0 & $\mathrm{X}$ & $\mathrm{X}$ & $\mathrm{X}$ & 0 & $\mathrm{X}$ & $\mathrm{X}$ & 0 & 0 & 0 & 0 & 0 & 0 \\
\hline & 3 & 0 & + & 0 & 0 & 0 & $\mathrm{X}$ & $\mathrm{X}$ & $\mathrm{X}$ & 0 & $\mathrm{X}$ & $\mathrm{X}$ & 0 & 0 & 0 & 0 & 0 & 0 \\
\hline & 4 & 0 & 0 & 0 & + & 0 & $\mathrm{X}$ & $\mathrm{X}$ & $X$ & 0 & $X$ & $x$ & 0 & 0 & 0 & 0 & 0 & 0 \\
\hline & 5 & 0 & 0 & 0 & + & - & $X$ & $X$ & $X$ & 0 & $X$ & $X$ & $+^{*}$ & $+^{*}$ & 0 & - & 0 & 0 \\
\hline & 6 & 0 & 0 & 0 & + & - & $X$ & $X$ & $X$ & 0 & $x$ & $x$ & $+^{*}$ & 0 & 0 & - & 0 & 0 \\
\hline & 7 & + & + & + & + & $+\mathrm{R}$ & $\mathrm{CC}$ & + & 0 & 0 & + & - & -* $^{*}$ & $\mathrm{CL}$ & + & - & $-\mathrm{R}$ & 0 \\
\hline & 8 & + & + & 0 & + & $+\mathrm{R}$ & - & - & 0 & 0 & $x$ & $\mathrm{CL}$ & 0 & 0 & $\mathrm{CL}$ & 0 & 0 & 0 \\
\hline & 9 & + & 0 & 0 & + & $+\mathrm{R}$ & + & 0 & 0 & 0 & $\mathrm{CL}$ & - & - & 0 & + & $\mathrm{CL}$ & 0 & 0 \\
\hline & 10 & - & 0 & + & + & $-\mathrm{R}$ & $\mathrm{CL}$ & $x$ & $x$ & 0 & $x$ & $X$ & $-^{*}$ & $\mathrm{CC}$ & + & - & - & + \\
\hline & 11 & - & - & 0 & - & 0 & $x$ & $x$ & $x$ & 0 & $X$ & $\mathrm{CL}$ & $-^{*}$ & - & CC & + & - & + \\
\hline & 12 & - & + & 0 & 0 & 0 & + & $\mathrm{CC}$ & 0 & 0 & - & + & 0 & 0 & $-R$ & 0 & $\mathrm{CL}$ & 0 \\
\hline & 13 & - & + & + & + & + & $x$ & $X$ & $X$ & 0 & $X$ & $x$ & $-^{*}$ & + & $+\mathrm{R}$ & - & $-\mathrm{R}$ & 0 \\
\hline & 14 & + & + & 0 & 0 & 0 & $X$ & $\mathrm{CL}$ & $X$ & 0 & $X$ & $x$ & 0 & 0 & $-\mathrm{R}$ & + & CC & 0 \\
\hline & 15 & + & + & 0 & - & 0 & - & + & $\mathrm{CL}$ & 0 & 0 & - & 0 & 0 & $-\mathrm{R}$ & + & $-\mathrm{R}$ & $\mathrm{CL}$ \\
\hline & 16 & - & + & + & + & - & $x$ & $X$ & $x$ & 0 & $X$ & $x$ & $-{ }^{*}$ & + & $+\mathrm{R}$ & - & $-\mathrm{R}$ & 0 \\
\hline & 17 & + & + & 0 & + & $-\mathrm{R}$ & 0 & - & CC & 0 & 0 & - & 0 & 0 & $-\mathrm{R}$ & + & $-\mathrm{R}$ & CC \\
\hline & 18 & - & + & 0 & - & $-R$ & $x$ & $X$ & $X$ & $\mathrm{CC}$ & $x$ & $x$ & 0 & - & $+\mathrm{R}$ & + & 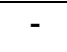 & 0 \\
\hline & 19 & + & + & 0 & + & $+\mathrm{R}$ & $X$ & $X$ & $X$ & $\mathrm{CL}$ & $X$ & $X$ & 0 & - & $+\mathrm{R}$ & + & - & 0 \\
\hline & 20 & + & + & 0 & + & 0 & $X$ & $x$ & $X$ & 0 & $X$ & $x$ & 0 & + & - & - & - & 0 \\
\hline & 21 & - & $-^{*}$ & 0 & - & 0 & $x$ & $X$ & $X$ & 0 & $X$ & $x$ & + & 0 & - & + & + & 0 \\
\hline & 22 & - & - & 0 & + & - & $X$ & $x$ & $x$ & 0 & $x$ & $x$ & 0 & - & + & + & 0 & 0 \\
\hline
\end{tabular}

Table 6.7 shows the final $1 / O$ effect matrix observed with all regulatory controllers implemented. 'CL' indicates a Closed Loop, 'CC' indicates a Cascade Control and ' $R$ ' indicates a regulated response, i.e., a movement of the manipulated variable or setpoint causes a change in the measured variable (positive or negative), but the variable returns to the previous value after a while (this indicates a null steadystate gain with a transient dynamic disturbance response). 
It is observed that the matrix changes when the controllers are operational, which is due to the coupling and uncoupling effects of the PID controllers in the process.

Now, with a model available for the complete process, a new problem is formulated for the Optimal Multi-Objective Technique for Integrated Control Structure Selection and Controller Tuning, considering all measurable and all manipulated variables and using the current control design as initial condition. In this step, the stabilizing loops are opened and rebuilt, using cascade controls. This is due to the fact that the manipulated variables selected for the stabilizing control intensely affect some other variables, being the cascade structure a better option for a faster control.

The unstable output variables are optimized to be kept at their setpoints, the feed flows are optimized to present minimum variability and the remaining variables are screened to optimize overall stability and minimize operational cost. After the optimal pairing is solved, the resulting configuration is as follows:

1. XMEAS 1 (A feed) $>$ XMV 3 (A feed flow);

2. XMEAS 2 ( $D$ feed) $>$ XMV 1 (D feed flow);

3. XMEAS 3 (E feed) $>$ XMV 2 (E feed flow);

4. XMEAS 4 ( $A$ and $C$ feed) $>$ XMV 4 ( $A$ and $C$ feed flow);

5. $\quad$ XMEAS 7 (reactor pressure) $>$ XMEAS 10 (purge rate) $>$ XMV 6 (purge valve);

6. XMEAS 8 (reactor level) $>$ XMEAS 11 (product separator temperature) $>$ XMV 11 (condenser cooling water flow);

7. XMEAS 9 (reactor temperature) $>$ XMV 10 reactor cooling water flow);

8. XMEAS 12 (product separator level) > XMEAS 14 (product separator underflow) > XMV 7 (separator pot liquid flow);

9. XMEAS 15 (stripper level) > XMEAS 17 (stripper underflow) > XMV 8 (stripper liquid product flow);

10. XMEAS 18 (stripper temperature) $>$ XMEAS 19 (stripper steam flow) $>$ XMV 9 (stripper steam valve);

11. XMEAS 21 (reactor cooling water outlet temperature) > XMV 12 (agitator speed).

The final I/O pairing for the regulatory control layer and the respective identified models and controller tunings are presented in Table 6.8. All PID controllers are 
designed to operate at a sampling time of 1 second. For better clarity, only the final pairings are shown (alternative candidates and models for unused relations are omitted).

Table 6.8 - I/O Pairings and PID tunings ( $T s=1 \mathrm{~s}$ ) for the regulatory control layer.

\begin{tabular}{|c|c|c|c|}
\hline $\begin{array}{c}\text { Controlled } \\
\text { Variable }\end{array}$ & $\begin{array}{l}\text { Manipulated } \\
\text { Variable }\end{array}$ & Identified Model & PID Tuning \\
\hline XMEAS 1 & XMV 3 & $G(s)=\frac{0.0017}{s-0.16}$ & $\begin{array}{l}K_{c}=82 \\
T_{i}=5.3\end{array}$ \\
\hline XMEAS 2 & XMV 1 & $G(s)=\frac{7.45}{s+0.13}$ & $\begin{array}{r}K_{c}=0.036 \\
T_{i}=7.75\end{array}$ \\
\hline XMEAS 3 & XMV 2 & $G(s)=\frac{7.45}{s+0.13}$ & $\begin{array}{r}K_{c}=0.036 \\
T_{i}=7.75 \\
\end{array}$ \\
\hline XMEAS 4 & XMV 4 & $G(s)=\frac{0.017}{s+0.11}$ & $\begin{array}{r}K_{c}=8 \\
T_{i}=15\end{array}$ \\
\hline XMEAS 7 & SP 10 & $G(s)=\frac{-0.15 s^{2}-0.014 s-0.00031}{s^{3}+0.027 s^{2}+0.0029 s}$ & $\begin{array}{r}K_{c}=-0.01 \\
T_{i}=3600 \\
\end{array}$ \\
\hline XMEAS 8 & SP 11 & $G(s)=\frac{(-5.48 s-0.19) \cdot 10^{-5}}{s^{2}+0.0019 s}$ & $\begin{array}{l}K_{c}=1.1 \\
T_{i}=600\end{array}$ \\
\hline XMEAS 9 & XMV 10 & $G(s)=\frac{-0.0050 \mathrm{~s}-0.0000047}{\mathrm{~s}^{2}+0.00053 \mathrm{~s}+0.0000025}$ & $\begin{array}{r}K_{c}=-4.5 \\
T_{i}=600 \\
\end{array}$ \\
\hline XMEAS 10 & XMV 6 & $G(s)=\frac{0.0013}{s+0.15}$ & $\begin{array}{r}K_{c}=7.65 \\
T_{i}=10\end{array}$ \\
\hline XMEAS 11 & XMV 11 & $G(s)=\frac{-0.0021 \mathrm{~s}-0.000000092}{\mathrm{~s}^{2}+0.0022 s+0.00000045}$ & $\begin{array}{l}K_{c}=-1 \\
T_{i}=500\end{array}$ \\
\hline XMEAS 12 & SP 14 & $G(s)=\frac{-0.185}{s}$ & $\begin{array}{l}K_{c}=-0.3 \\
T_{i}=3600\end{array}$ \\
\hline XMEAS 14 & XMV 7 & $G(s)=\frac{0.025}{s+0.038}$ & $\begin{array}{r}K_{c}=0.7 \\
T_{i}=6\end{array}$ \\
\hline XMEAS 15 & SP 17 & $G(s)=-\frac{0.0039}{s}$ & $\begin{array}{r}K_{c}=-0.2 \\
T_{i}=600\end{array}$ \\
\hline XMEAS 17 & XMV 8 & $G(s)=\frac{0.10}{s+0.22}$ & $\begin{array}{r}K_{c}=0.6 \\
T_{i}=2 \\
\end{array}$ \\
\hline XMEAS 18 & SP 19 & $G(s)=\frac{\left(0.40 s^{2}+0.016 s\right) \cdot 10^{-4}}{s^{2}+0.017 s+0.000071}$ & $\begin{array}{r}K_{c}=50 \\
T_{i}=1800 \\
\end{array}$ \\
\hline XMEAS 19 & XMV 9 & $G(s)=\frac{0.051}{s+0.012}$ & $\begin{array}{r}K_{c}=0.15 \\
T_{i}=120\end{array}$ \\
\hline XMEAS 21 & XMV 12 & $G(s)=\frac{0.00061 s+0.00012}{s^{2}+0.017 s+0.0012}$ & $\begin{array}{r}K_{c}=30 \\
T_{i}=1800\end{array}$ \\
\hline
\end{tabular}

With this initial configuration, the following 16 output variables are controlled by the regulatory layer: XMEAS 1, XMEAS 2, XMEAS 3, XMEAS 4, XMEAS 7, XMEAS 8, 
XMEAS 9, XMEAS 10, XMEAS 11, XMEAS 12, XMEAS 14, XMEAS 15, XMEAS 17, XMEAS 18, XMEAS 19 and XMEAS 21. This leaves the following 6 variables uncontrolled by this layer: XMEAS 5, XMEAS 6, XMEAS 13, XMEAS 16, XMEAS 20 and XMEAS 22. Such variables will need to be dealt with in the design of the supervisory layer.

\section{Implementation of the Regulatory Control Layer}

The designed controllers (optimized for the process model) are then implemented. Figure 6.4 shows the plant with the complete regulatory control layer implemented in Simulink.

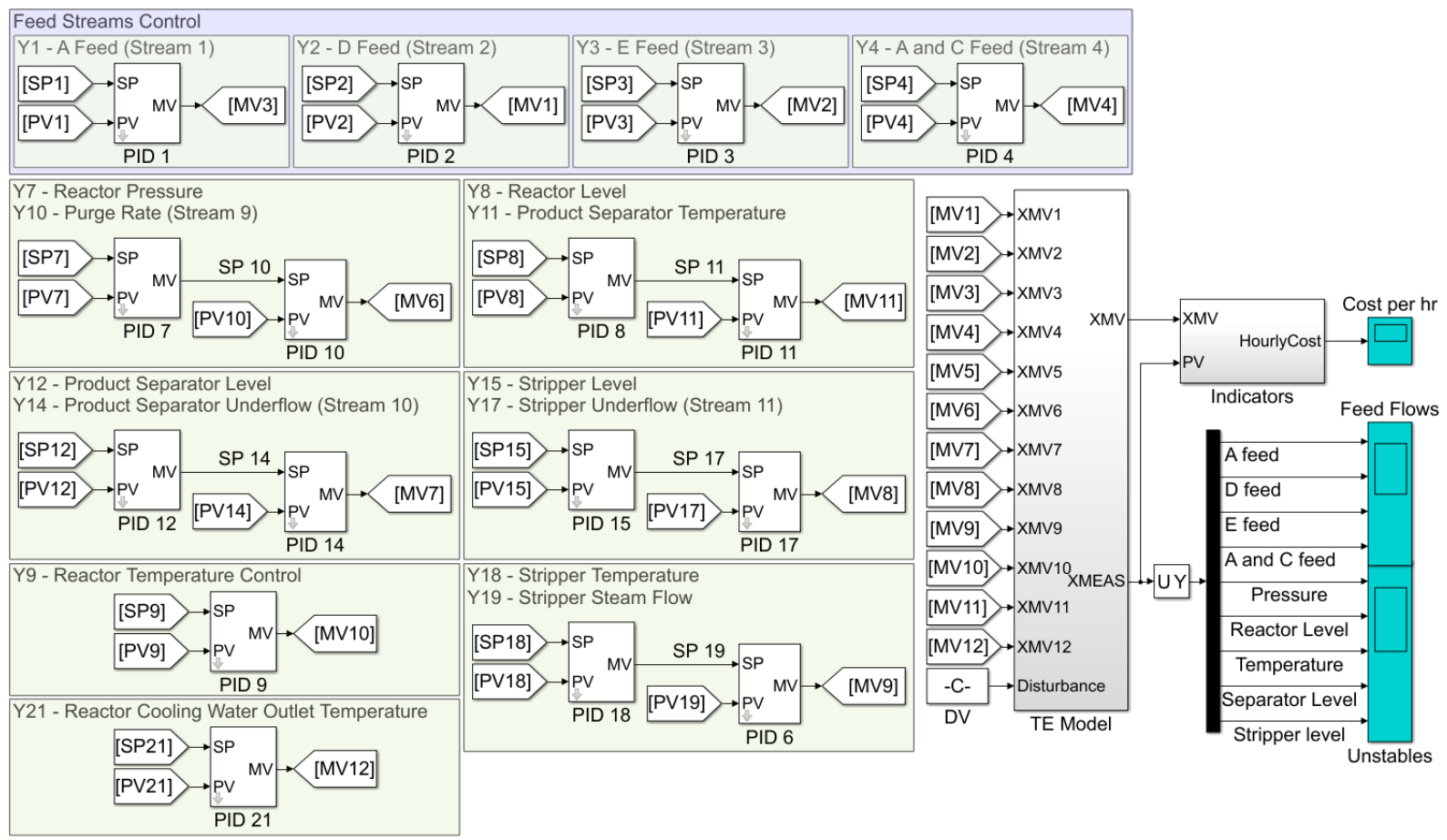

Figure 6.4 - Tennessee Eastman Challenge with regulatory control.

Performance tests are carried out with the process at nominal operating point to validate the designed control system. Finally, minor adjustments are made to the tuning parameters to reduce overshoot and oscillations.

At this point, the process is stable and has a regulatory layer of PID controllers to follow setpoint changes and reject disturbances. The plant can now successfully operate, respecting operational constraints and following setpoints. Process responses are shown in Subsection 6.7. 


\section{Supervisory Control Layer}

Although the regulatory control layer is capable of satisfactorily controlling the plant, a MPC controller may be beneficial for this process, due to the following reasons:

> The MPC is a multivariable controller, which allows it to compensate the undesired effects seen in other controlled variables, when a manipulated variable is moved to adjust a controlled variable;

$>\quad$ The MPC controller employs a process model to predict future states of the plant, allowing it to optimize trajectories based on such predictions;

$>\quad$ This process is very large and complex, which makes it a very good candidate to be improved by an MPC controller.

The first step in the design of the supervisory control is to obtain a detailed dynamic model of the process, including all the regulatory layer controllers operating in closed-loop.

First, simple step tests are performed and the process response is used to create an I/O effect matrix for the process in closed loop.

Next, an identification experiment is performed, with pseudo-random binary excitation signals applied to all 11 setpoints of the regulatory controllers and to the free manipulated variable (XMV 5).

Then, dynamic models are identified for all input-output relations and a single MIMO model with 12 inputs and 22 outputs and sampling time of 1 minute is built.

Next, the control specifications for the supervisory layer are compiled based on decisions taken in the previous steps and on the newly acquired model. XMEAS 8, XMEAS 10, XMEAS 19 and XMEAS 20 directly affect the operation cost and should be optimized by the MPC controller. It is verified that most of the other variables indirectly affect the operational cost too, even if they are not explicitly computed in the cost, and should be optimized as well.

The initial choice for the supervisory layer is to split the process between two multivariable controllers, one for the reactor unit (manipulating SP 1, SP 2, SP 3, SP 7, SP 8 and SP 9), and another for the other units (manipulating SP 4, SP 12, SP 15, 
SP 18 and XMV 5). As will be shown later, this choice was changed for a single controller for the complete process.

It is also determined that the controller tuning for this layer should be performed to optimize the operational cost function, followed by the minimization of the output deviation from the setpoints and the minimization of the amplitude of the movements of the manipulated variables.

The control layer is then designed and tuned, with different options evaluated. At the end, a single MPC controller it is chosen to be used in the supervisory layer, which is also used to optimize the plantwide operating cost.

Each part of the design is detailed next.

\section{Closed-Loop I/O Effect Matrix}

The first step of the design of the supervisory control layer is to determine the I/O effect matrix for the process with regulatory control. For that, short experiments with step sequences are applied to the candidate manipulated variables (the setpoints for the regulatory layer and XMV 5).

The collected data is used to determine the new $/ \mathrm{O}$ effect matrix and it is also prepared to be employed as validation data in the identification. The resulting matrix is presented in Table 6.9. 
Table $6.9-1 / 0$ effect matrix for the process with active regulatory controllers.

\begin{tabular}{|c|c|c|c|c|c|c|c|c|c|c|c|c|c|}
\hline & \multicolumn{11}{|c|}{ Setpoints (SP) } & \multirow{2}{*}{$\begin{array}{c}\mathrm{XMV} \\
5\end{array}$} \\
\hline & & 1 & 2 & 3 & 4 & 7 & 8 & 9 & 12 & 15 & 18 & 21 & \\
\hline \multirow{22}{*}{ 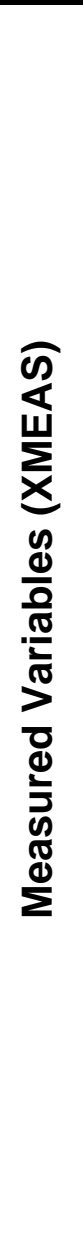 } & 1 & $\overline{\mathrm{CL}}$ & $\overline{0}$ & 0 & 0 & 0 & $\overline{0}$ & $\overline{0}$ & 0 & $\overline{0}$ & 0 & 0 & $\overline{0}$ \\
\hline & 2 & 0 & $\mathrm{CL}$ & 0 & 0 & 0 & 0 & 0 & 0 & 0 & 0 & 0 & 0 \\
\hline & 3 & 0 & 0 & $\mathrm{CL}$ & 0 & 0 & 0 & 0 & 0 & 0 & 0 & 0 & 0 \\
\hline & 4 & 0 & 0 & 0 & $\mathrm{CL}$ & 0 & 0 & 0 & 0 & 0 & 0 & 0 & 0 \\
\hline & 5 & $0^{*}$ & - & - & + & + & 0 & - & 0 & 0 & 0 & - & + \\
\hline & 6 & 0 & - & - & + & + & 0 & - & 0 & 0 & 0 & - & + \\
\hline & 7 & $+\mathrm{R}$ & $-\mathrm{R}$ & $+\mathrm{R}$ & $+\mathrm{R}$ & $\mathrm{CL}$ & $+\mathrm{R}$ & $-\mathrm{R}$ & $+\mathrm{R}$ & $+\mathrm{R}$ & 0 & 0 & $-R$ \\
\hline & 8 & $-R$ & + & + & - & 0 & $\mathrm{CL}$ & $-\mathrm{R}$ & 0 & 0 & 0 & 0 & 0 \\
\hline & 9 & 0 & $+\mathrm{R}$ & 0 & 0 & 0 & $+\mathrm{R}$ & $\mathrm{CL}$ & 0 & 0 & 0 & 0 & 0 \\
\hline & 10 & + & - & + & + & CC & + & - & 0 & 0 & 0 & 0 & 0 \\
\hline & 11 & 0 & - & - & + & + & $\mathrm{CC}$ & + & + & - & 0 & - & + \\
\hline & 12 & 0 & 0 & 0 & 0 & 0 & 0 & 0 & $\mathrm{CL}$ & 0 & 0 & 0 & 0 \\
\hline & 13 & $+R$ & $-R$ & $+\mathrm{R}$ & $+\mathrm{R}$ & + & $+\mathrm{R}$ & $-R$ & $+\mathrm{R}$ & $+\mathrm{R}$ & 0 & 0 & - \\
\hline & 14 & 0 & + & + & - & 0 & $+R$ & $+\mathrm{R}$ & CC & 0 & 0 & 0 & 0 \\
\hline & 15 & 0 & 0 & $+\mathrm{R}$ & 0 & 0 & $-R$ & $+\mathrm{R}$ & $-R$ & $\mathrm{CL}$ & 0 & 0 & 0 \\
\hline & 16 & $+R$ & $-R$ & + & $+\mathrm{R}$ & + & $+\mathrm{R}$ & $-R$ & $+\mathrm{R}$ & $+\mathrm{R}$ & 0 & 0 & + \\
\hline & 17 & 0 & + & + & 0 & 0 & $-R$ & $+\mathrm{R}$ & $-R$ & CC & 0 & 0 & 0 \\
\hline & 18 & 0 & $-R$ & - & $-R$ & 0 & $+R$ & + & $-R$ & 0 & $\mathrm{CL}$ & 0 & 0 \\
\hline & 19 & 0 & + & + & - & - & $-R$ & - & + & - & CC & - & - \\
\hline & 20 & $0^{*}$ & - & - & + & + & 0 & + & + & + & + & - & - \\
\hline & 21 & 0 & $-R$ & 0 & 0 & 0 & $-R$ & $+\mathrm{R}$ & 0 & 0 & 0 & $\mathrm{CL}$ & 0 \\
\hline & 22 & $0^{*}$ & - & - & + & + & $+R$ & + & + & + & + & - & + \\
\hline
\end{tabular}

\section{System Identification}

With the $1 / O$ effect matrix built, a new set of multivariable identification experiments is designed. Two experiments are designed to minimize interference between the variables, with the following group of inputs being excited in each experiment:

D Experiment 1: SP 1, SP 2, SP 7, SP 9, SP 12, XMV 5;

$>$ Experiment 2: SP 3, SP 4, SP 8, SP 15, SP 18, SP 21.

Sets of uncorrelated GBN signals are generated for each experiment and applied to the process. 
The data collected is then employed to identify models for the process in closed loop (with the regulatory controllers active). MISO models are obtained for each output in each experiment, totalizing 44 complete identifications.

For all identifications, the CASI toolbox is employed. The first step is the preparation and pre-processing of the data. The resulting datasets are then given to the OSOD (Optimal Selection of Order and Delay) algorithm, which determines the best model order and time delay for each of the 29 model structures available in the toolbox. The resulting models (1276 in total) are validated and the poor models are excluded. In the final identification step, the good models are inspected and the best model for each dataset is selected. This results in $88 \mathrm{MISO}$ models obtained from the two identification experiments performed. A complete identification procedure for one dataset is presented and detailed in Appendix A.

\section{MIMO Process Model}

With all models identified, a model fusion needs to be performed to build a single model to be used in the MPC controller.

Each model was identified with the best sampling time for each output. For the fusion of the models, all of them are resampled to 1 minute sampling time, the sampling time to be used by the MPC controller.

Each of the models were also generated in a structure most suitable for the corresponding variables. For unifying the models, all of them were converted to the BJ structure. It is important to note that this conversion is lossless, and all models retain all its dynamic characteristics.

Next, the models for each experiment are converted to a single $12 \times 22$ denormalized BJ multivariable model.

For the fusion of models from both experiments, the process models are concatenated and the disturbance model with highest gain is retained for each output.

The final MIMO model is validated using the complete datasets from each experiment, with models and data in engineering units. This is performed to assess if any error was made in the model conversions performed. 
The final identified model is presented in Appendix B.

\section{MPC Design}

After the system identification is completed, the MPC controller needs to be designed. It is decided to evaluate the performance of the system with a single MPC controller. The Model Predictive Control Toolbox of MATLAB ${ }^{\circledR}$ is used for this design.

Fist, the multivariable model is used to build an MPC controller to control the model itself. Nominal values for each input and output, as shown in Table 6.2, are configured in the controllers, as well as scale factors to normalize the variables to the performance function. The scale factors used for the MPC manipulated variables are shown in Table 6.10 and the scale factors used for the output variables are shown in Table 6.11.

Table 6.10 - Scale factors for the MPC manipulated variables.

\begin{tabular}{c|c|c}
\hline MPC Variable & Corresponding Process Variable & Scale Factor \\
\hline MV 1 & SP 1 & 0.4 \\
\hline MV 2 & SP 2 & 732.8 \\
\hline MV 3 & SP 3 & 450.93 \\
\hline MV 4 & SP 4 & 0.93477 \\
\hline MV 5 & SP 7 & 40 \\
\hline MV 6 & SP 8 & 20 \\
\hline MV 7 & SP 9 & 10 \\
\hline MV 8 & SP 12 & 20 \\
\hline MV 9 & SP 15 & 13.1462 \\
\hline MV 10 & SP 18 & 6 \\
\hline MV 11 & SP 21 & 10 \\
\hline MV 12 & XMV 5 &
\end{tabular}


Table 6.11 - Scale factors for the output variables used in the MPC controller.

\begin{tabular}{c|c|c}
\hline MPC Variable & Corresponding Process Variable & Scale Factor \\
\hline OV 1 & XMEAS 1 & 0.4 \\
\hline OV 2 & XMEAS 2 & 732.8 \\
\hline OV 3 & XMEAS 3 & 450.93 \\
\hline OV 4 & XMEAS 4 & 0.93477 \\
\hline OV 5 & XMEAS 5 & 10 \\
\hline OV 6 & XMEAS 6 & 10 \\
\hline OV 7 & XMEAS 7 & 40 \\
\hline OV 8 & XMEAS 8 & 20 \\
\hline OV 9 & XMEAS 9 & 10 \\
\hline OV 10 & XMEAS 10 & 1 \\
\hline OV 11 & XMEAS 11 & 20 \\
\hline OV 12 & XMEAS 12 & 400 \\
\hline OV 13 & XMEAS 13 & 40 \\
\hline OV 14 & XMEAS 14 & 20 \\
\hline OV 15 & XMEAS 15 & 500 \\
\hline OV 16 & XMEAS 16 & 40 \\
\hline OV 17 & XMEAS 17 & 450 \\
\hline OV 18 & XMEAS 18 & 90 \\
\hline OV 19 & XMEAS 19 & 60 \\
\hline OV 20 & XMEAS 20 & 4021 \\
\hline OV 21 & XMEAS 22 & \\
\hline OV 22 & & 40 \\
\hline
\end{tabular}

Based on the information previously gathered, constraints are also configured for each output and input, as well as for the input variation rate limits. Constraints used for the output variables are shown in Table 6.12 and constraints used for the input variables are shown in Table 6.13. 
Table 6.12 - MPC constraints for the controlled variables.

\begin{tabular}{l|c|c|c}
\hline \multicolumn{1}{c|}{ Variable Name } & $\begin{array}{c}\text { Variable } \\
\text { Number }\end{array}$ & $\begin{array}{c}\text { Minimum } \\
\text { Limit }\end{array}$ & $\begin{array}{c}\text { Maximum } \\
\text { Limit }\end{array}$ \\
\hline A feed (stream 1) & XMEAS 1 & 0 & 1 \\
\hline D feed (stream 2) & XMEAS 2 & 2500 & 5500 \\
\hline E feed (stream 3) & XMEAS 3 & 3500 & 5000 \\
\hline A and C feed (stream 4) & XMEAS 4 & 8 & 10 \\
\hline Recycle flow (stream 8) & XMEAS 5 & - & - \\
\hline Reactor feed rate (stream 6) & XMEAS 6 & - & - \\
\hline Reactor pressure & XMEAS 7 & 0 & 2895 \\
\hline Reactor level & XMEAS 8 & 55 & 100 \\
\hline Reactor temperature & XMEAS 9 & 0 & 150 \\
\hline Purge rate (stream 9) & XMEAS 10 & - & - \\
\hline Product separator temperature & XMEAS 11 & - & - \\
\hline Product separator level & XMEAS 12 & 30 & 100 \\
\hline Product separator pressure & XMEAS 13 & - & - \\
\hline Product separator underflow (stream 10) & XMEAS 14 & - & - \\
\hline Stripper level & XMEAS 15 & 30 & 100 \\
\hline Stripper pressure & XMEAS 16 & - & - \\
\hline Stripper underflow (stream 11) & XMEAS 17 & - & - \\
\hline Stripper temperature & XMEAS 18 & 50 & 80 \\
\hline Stripper steam flow & XMEAS 19 & - & - \\
\hline Compressor work & XMEAS 20 & - & - \\
\hline Reactor cooling water outlet temperature & XMEAS 21 & - & - \\
\hline Separator cooling water outlet temperature & XMEAS 22 & 70 & 100 \\
\hline & & & \\
\hline
\end{tabular}


Table 6.13 - Constraints for the MPC manipulated variables.

\begin{tabular}{l|c|c|c|c}
\hline $\begin{array}{c}\text { MPC Manipulated } \\
\text { Variable }\end{array}$ & $\begin{array}{c}\text { Minimum } \\
\text { Limit }\end{array}$ & $\begin{array}{c}\text { Maximum } \\
\text { Limit }\end{array}$ & $\begin{array}{c}\text { Minimum } \\
\text { Rate }\end{array}$ & $\begin{array}{c}\text { Maximum } \\
\text { Rate }\end{array}$ \\
\hline SP 1 & 0 & 1 & -0.5 & 0.5 \\
\hline SP 2 & 3000 & 4500 & -1000 & 1000 \\
\hline SP 3 & 4000 & 5000 & -1000 & 1000 \\
\hline SP 4 & 0 & 15 & -10 & 10 \\
\hline SP 7 & 0 & 3000 & -1 & 1 \\
\hline SP 8 & 50 & 100 & -50 & 50 \\
\hline SP 9 & 0 & 150 & -150 & 150 \\
\hline SP 12 & 30 & 100 & -70 & 70 \\
\hline SP 15 & 30 & 100 & -10 & 10 \\
\hline SP 18 & 0 & 100 & -0.1 & 0.1 \\
\hline SP 21 & 0 & 200 & -0.2 & 0.2 \\
\hline XMV 5 & 0 & 30 & -20 & 20 \\
\hline
\end{tabular}

The controller is then built with a sampling time of 1 minute and tuned based on the control specifications, using the proposed tuning procedure. The tuned parameters include the prediction and control horizons and weights for the output variables (OV Weights), for the input variables (MV Weights) and the rates of change of the input variables (MV Rate Weights). The tuning is performed to minimize the deviation from the setpoints for all 22 output variables, prioritizing first the gas feed (that should have minimum variability), then the variables that directly affect the cost function, then the unstable variables and at last the other outputs. The final tuning parameters are:

$>\quad$ Prediction horizon: 50;

$>\quad$ Control horizon: 7;

> OV Weights: [0.74 0.460.510.67000.770.620.76000.5800.880.5400 $0.88000 .530]$;

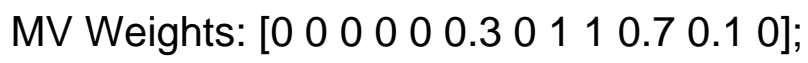

MV Rate Weights: [0.1 0.10 .10 .110 .30 .10 .10 .111 .0 0]. 


\section{MPC Implementation}

The designed MPC is then implemented in the plant. The Model Predictive Control Toolbox of MATLAB ${ }^{\circledR}$ is employed to implement the controller in the plant model.

The controller is inserted in the system, tested and the parameters are adjusted to improve the control performance. The first results show that a single controller can handle the complete process and provides a significantly better control than the process with only the regulatory PID controllers. At this point, it is decided to keep the single MPC controller for the supervisory control layer.

Figure 6.5 shows the process with regulatory and supervisory control layers implemented in Simulink.

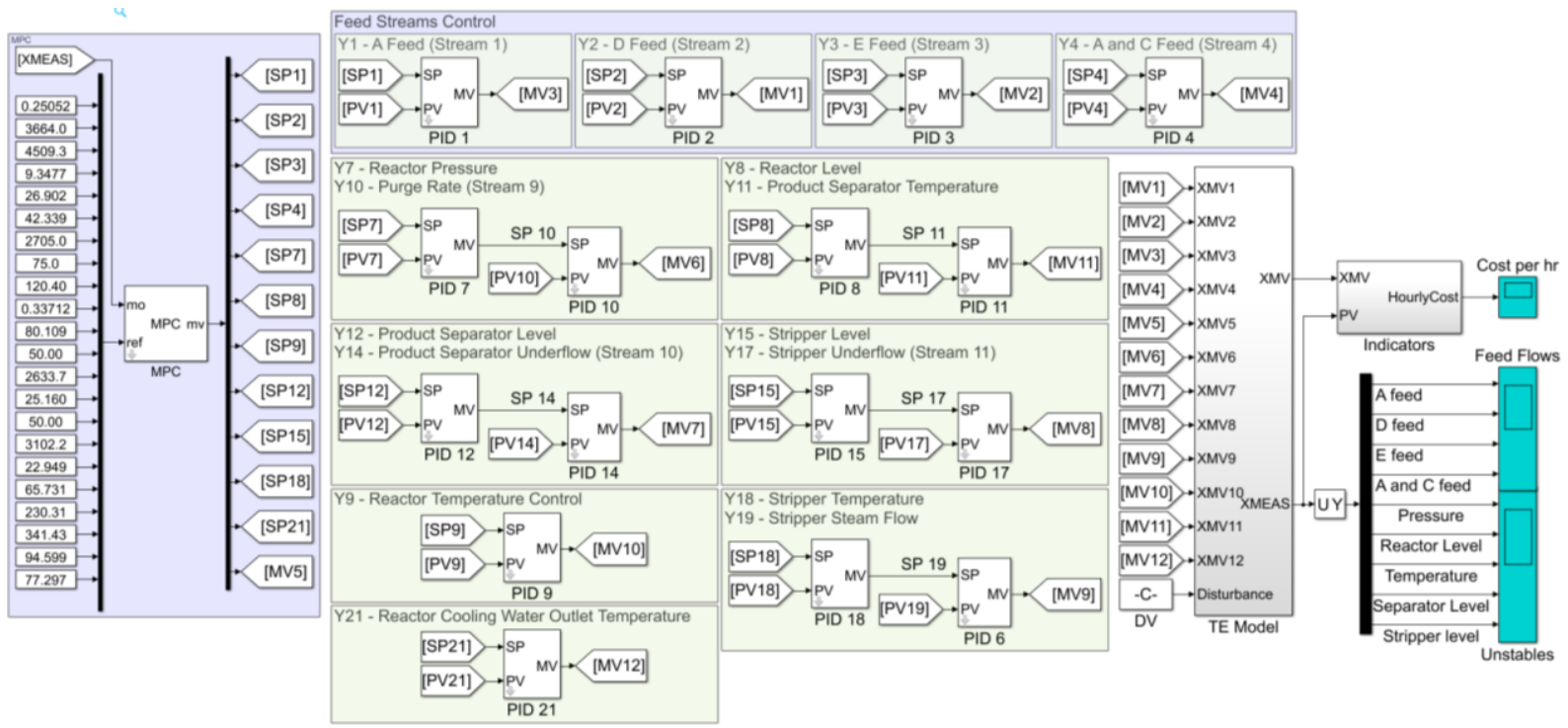

Figure 6.5 - Tennessee Eastman Challenge with regulatory and supervisory control layers.

Responses of the process with the final control system are shown in Subsection 6.7 and compared with those obtained when only a regulatory layer is present.

\section{Plantwide Control Layer}

Since a single MPC controller was able to handle the complete process, this same MPC is used to perform the plantwide economic optimization.

After all variables are stable, their variability minimized and the MPC controller is operating in the plant, the optimization of the operating cost needs to be added to 
the controller. This is done through the adjustment of the controller cost function weights.

First, the MPC controller tuning parameters that were adjusted for the plant are updated in the MPC controlling the identified model. Next, the operating cost function is added to the lexicographic tuning optimization problem for economic optimization. Then, a new optimization step is performed, to minimize the operating cost without causing significant loss in control performance.

The new MPC tuning is then updated in the plant and the system is tested, validated and adjusted to compensate model discrepancies from the process.

Final results are shown in Subsection 6.7.

This results in a control system that stabilizes the process, keeps it under operating constraints, keeps all controlled variables close to their setpoints and optimizes the operating cost of the process. The hierarchical plantwide control system is now complete.

\section{General Review and Enhancements}

The final step of the plantwide control design procedure is to review and evaluate the designed control system, making the necessary adjustments.

First, the complete control system is tested and evaluated. The behavior is considered satisfactory and the variable pairings and controllers' configurations are ratified.

For a final refinement of the control tuning, the following procedure is emulated: the system is put to operate continuously at the nominal operating point and the optimal multi-objective tuning technique is used to update the controllers' tuning parameters, after observing the system performance.

First, the PID tunings are updated after every few hours (depending on the dynamics of the variable being optimized) of operation. Most parameters are kept at their previously optimized values, with a few gains reduced to compensate oscillations and improve response times. 
Next, the MPC tuning parameters are revised. This is done with the parameters being reoptimized and updated after every 24 hours of operation.

These final adjustments complete the design procedure. A complete hierarchical plantwide control was designed and implemented for the Tennessee Eastman Challenge!

\subsection{Final Results}

Results of simulations of the Tennessee Eastman Challenge Process operating with the designed plantwide control system are shown along this section.

First, the responses of the gas feeds are presented. Next, the behavior of the unstable variables is shown, followed by the remaining key controlled variables.

\subsubsection{XMEAS 1, XMEAS 2, XMEAS 3 and XMEAS 4 (Gas Feeds)}

First, the feed flow controllers are evaluated. Figure 6.6 shows the process responses for a change in the feed flow setpoint values.
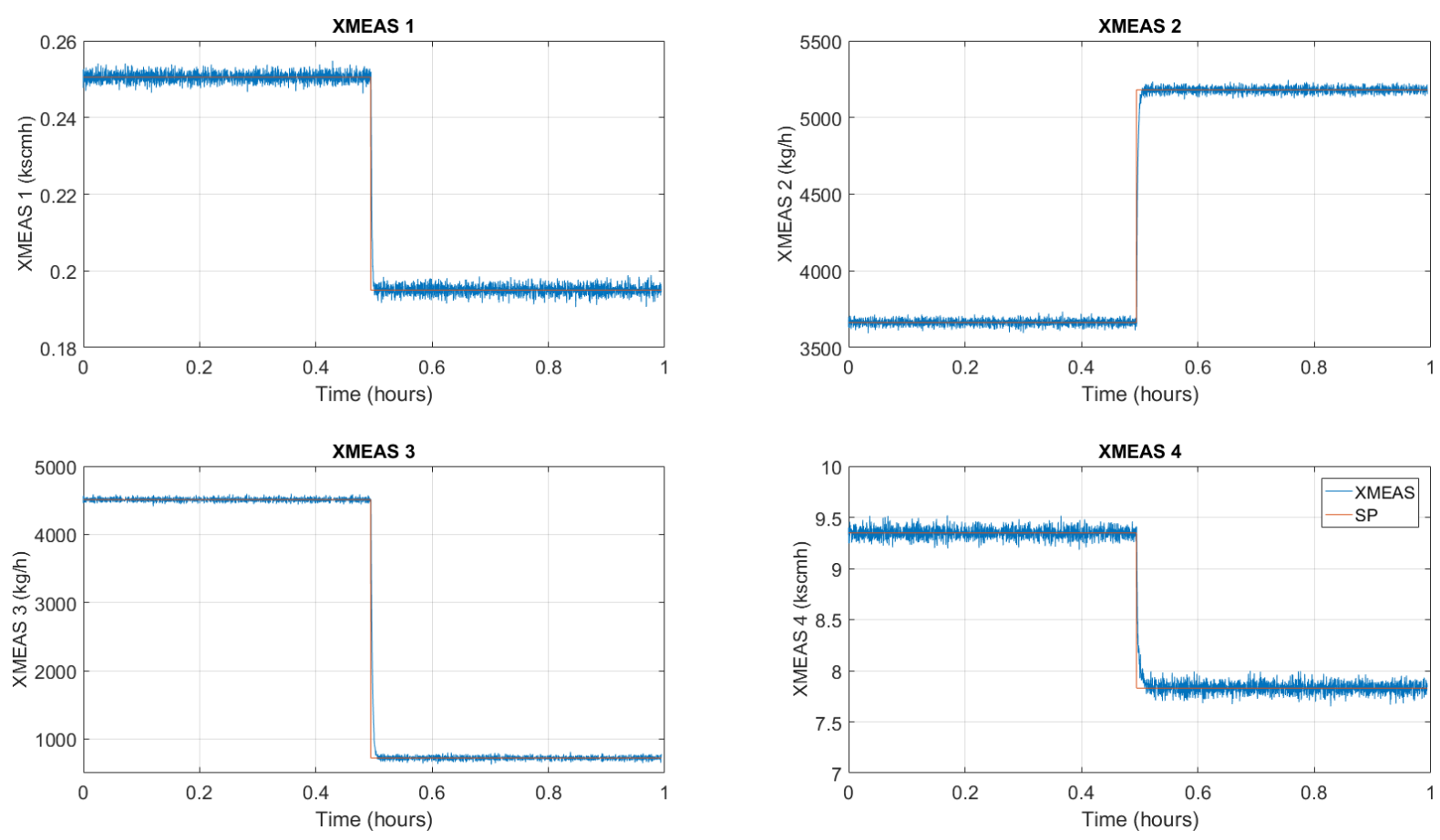

Figure 6.6 - Step responses for the feed flow controllers. 
As observed, the controllers are able to follow the setpoint with a fast and precise response.

Next, Figure 6.7 shows the feed flows for the process with the complete plantwide control active, with only the regulatory PID controllers active and with the feed flow control valves fixed at their nominal values.

It can be noted that the PID control presents a variability similar to the one observed with the XMV variables at fixed values. The MPC control uses these controlled variables in some instants to compensate disturbances, but the deviation from the setpoint is low.
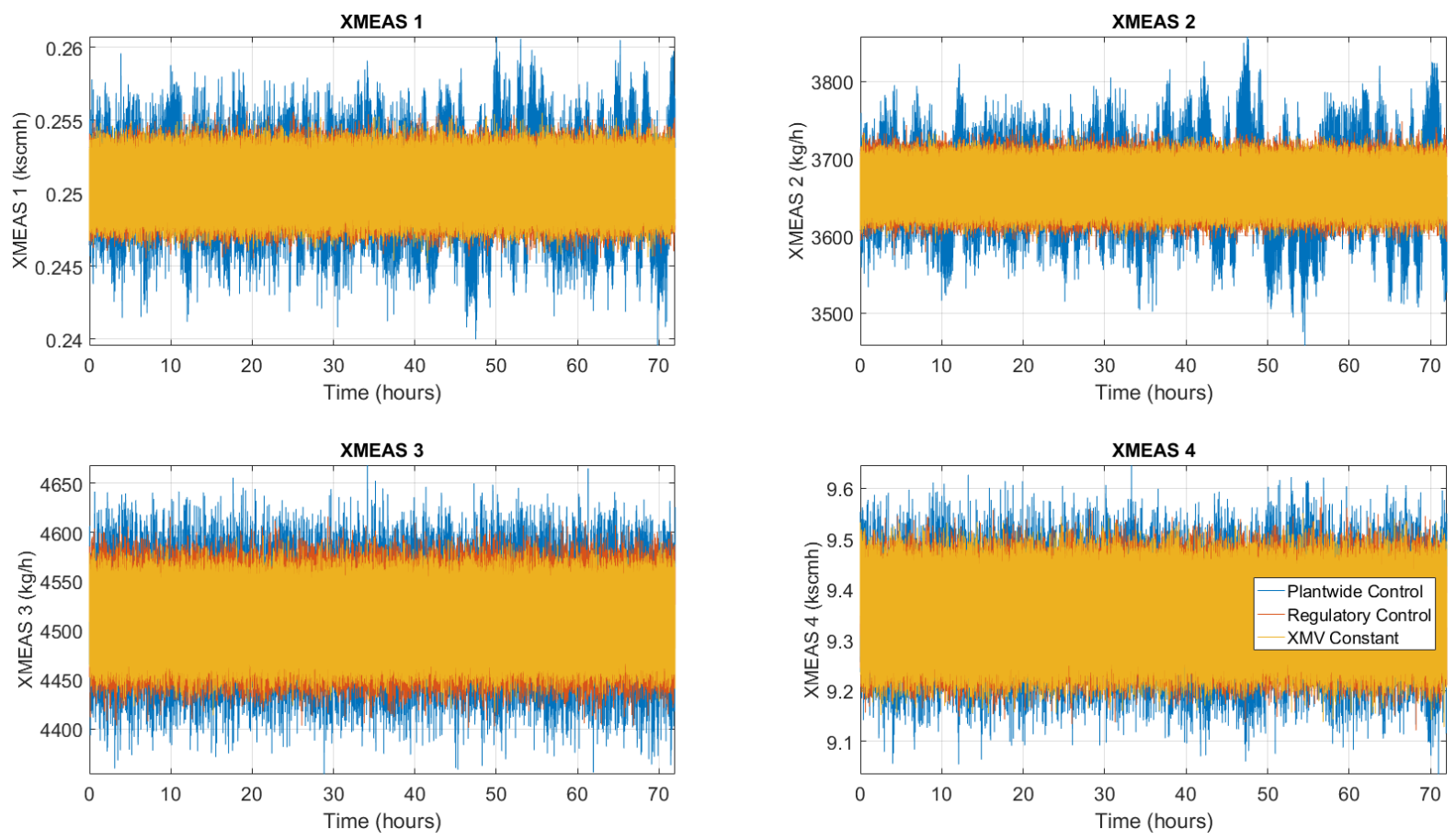

Figure 6.7 - Feed flow regulatory responses.

\subsubsection{XMEAS 7 (Reactor Pressure) and XMEAS 10 (Purge Rate)}

The next variables to be inspected are XMEAS 7 (reactor pressure) and XMEAS 10 (purge rate), which is used in a cascade control to regulate the reactor pressure.

Step responses for the XMEAS 7 are shown in Figure 6.8. As can be seen, both responses follow the setpoint, but the response with all control layers active is much faster and with a much better setpoint tracking. Moreover, the purge rate and purge valve observed in both scenarios present very similar characteristics. 
Regulatory responses for XMEAS 7 are shown in Figure 6.9.
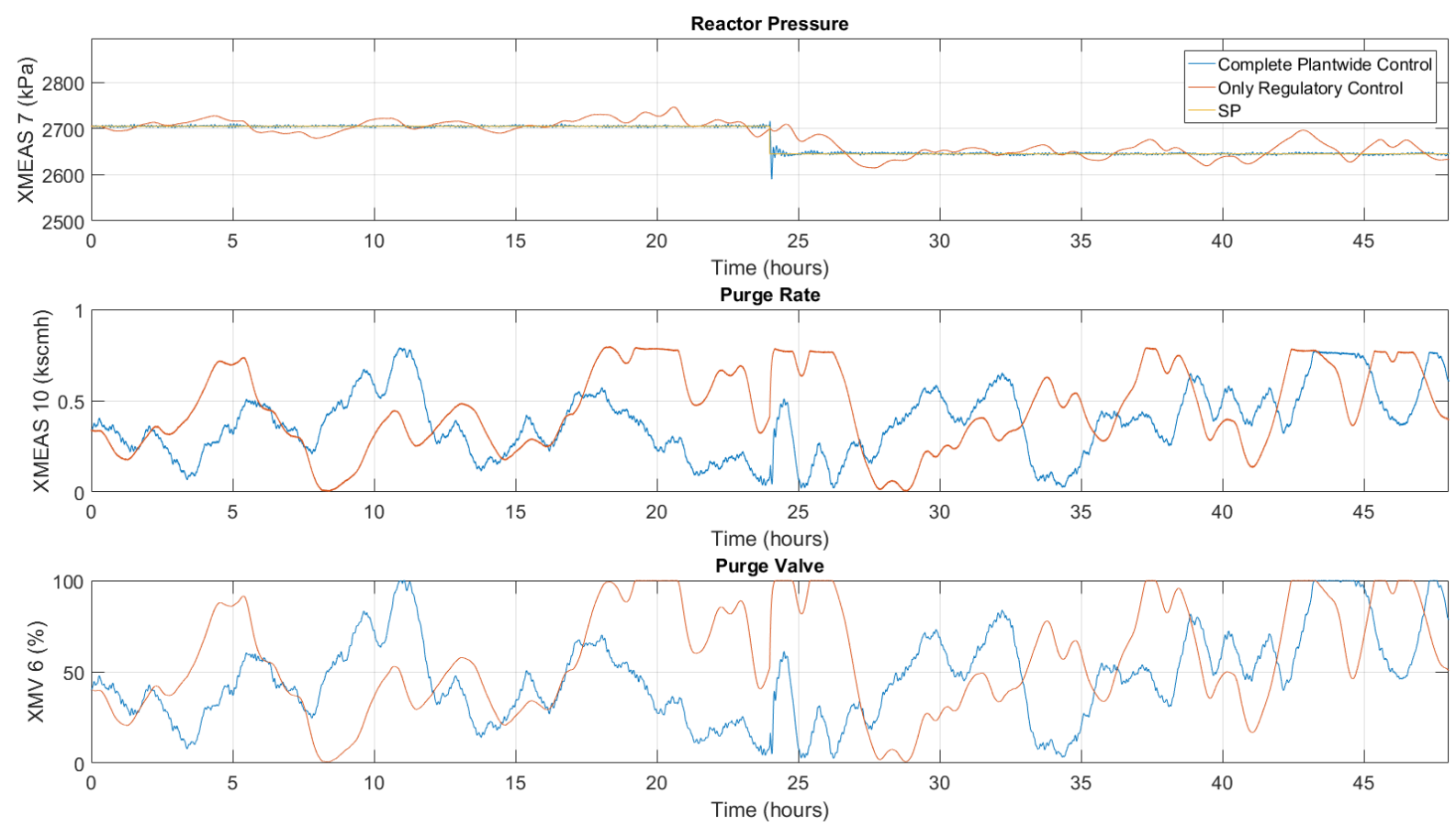

Figure 6.8 - Step response for XMEAS 7.
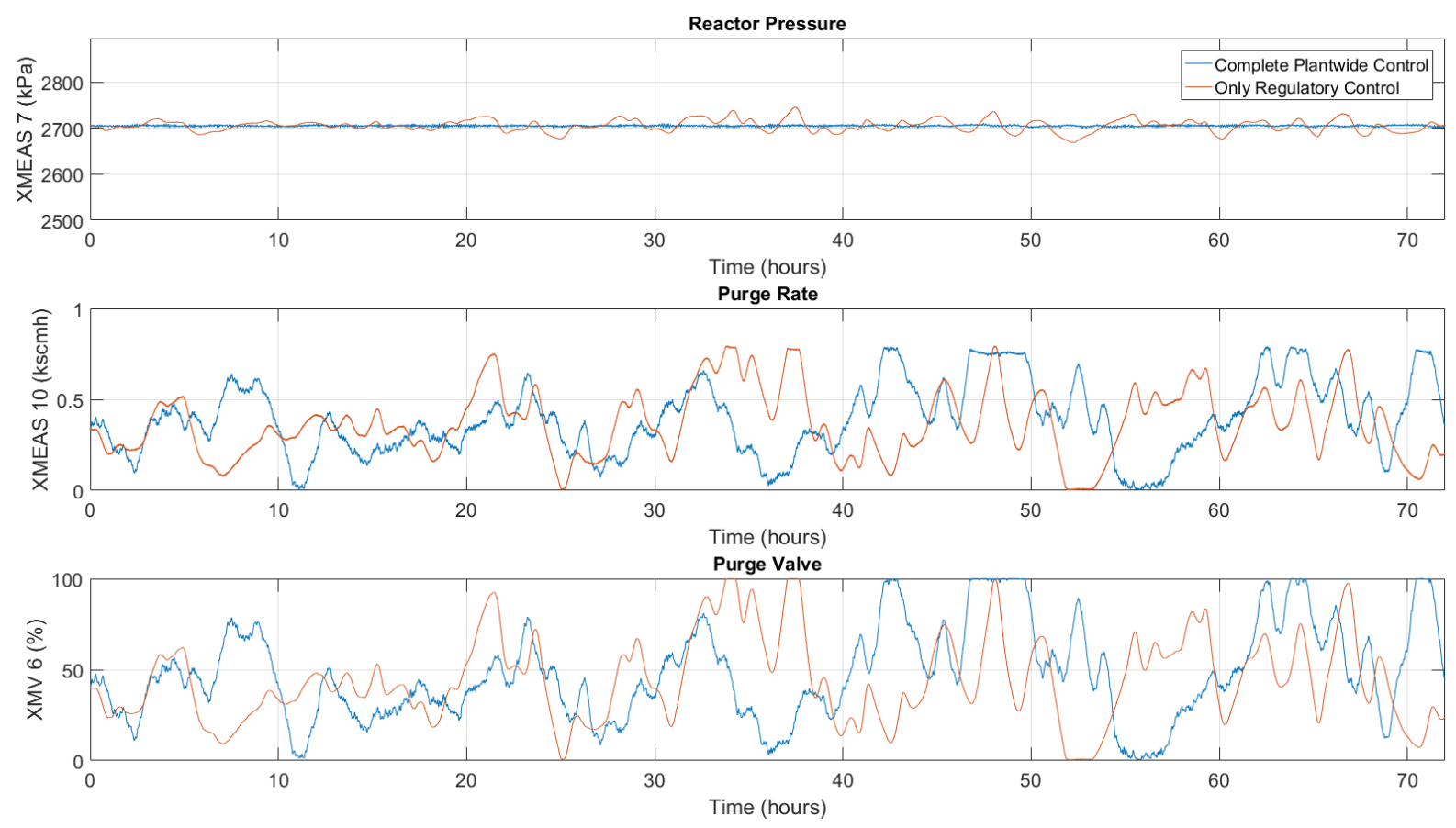

Figure 6.9 - Regulatory response for XMEAS 7 and XMEAS 10.

A control of good quality was achieved, with the system rejecting process disturbances and keeping the controlled variables at their setpoint. It can also be noted that the supervisory control layer greatly enhances the process response. 


\subsubsection{XMEAS 8 (Reactor Level) and XMEAS 11 (Product Separator Temperature)}

Figure 6.10 shows the response of the reactor level for a setpoint change from $75 \%$ to $80 \%$ and then to $70 \%$ and includes the behavior of the disturbed XMEAS 7 .
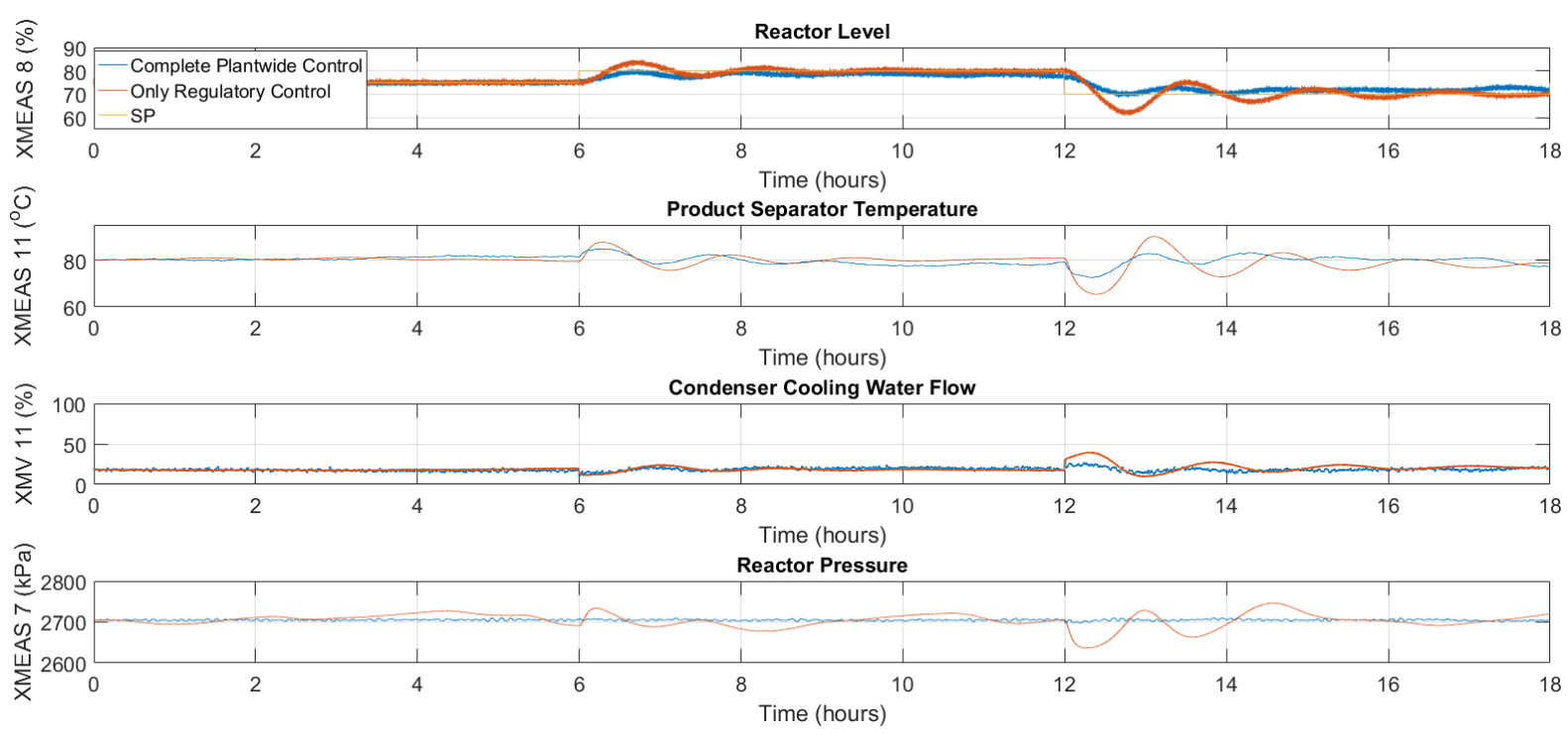

Figure 6.10 - Step Response for XMEAS 8.

Again, the plantwide architecture is able to follow setpoint changes and results in a smoother response when compared to independent PID controllers. Moreover, the multivariable control manages to change the reactor level without disturbing the pressure, which is one of the main advantages of such controller.

Next, Figure 6.11 shows the regulatory response for XMEAS 8 and XMEAS 11 for 72 hours of operation.

The control system manages to keep the variables under operating constraints and at their setpoints. 

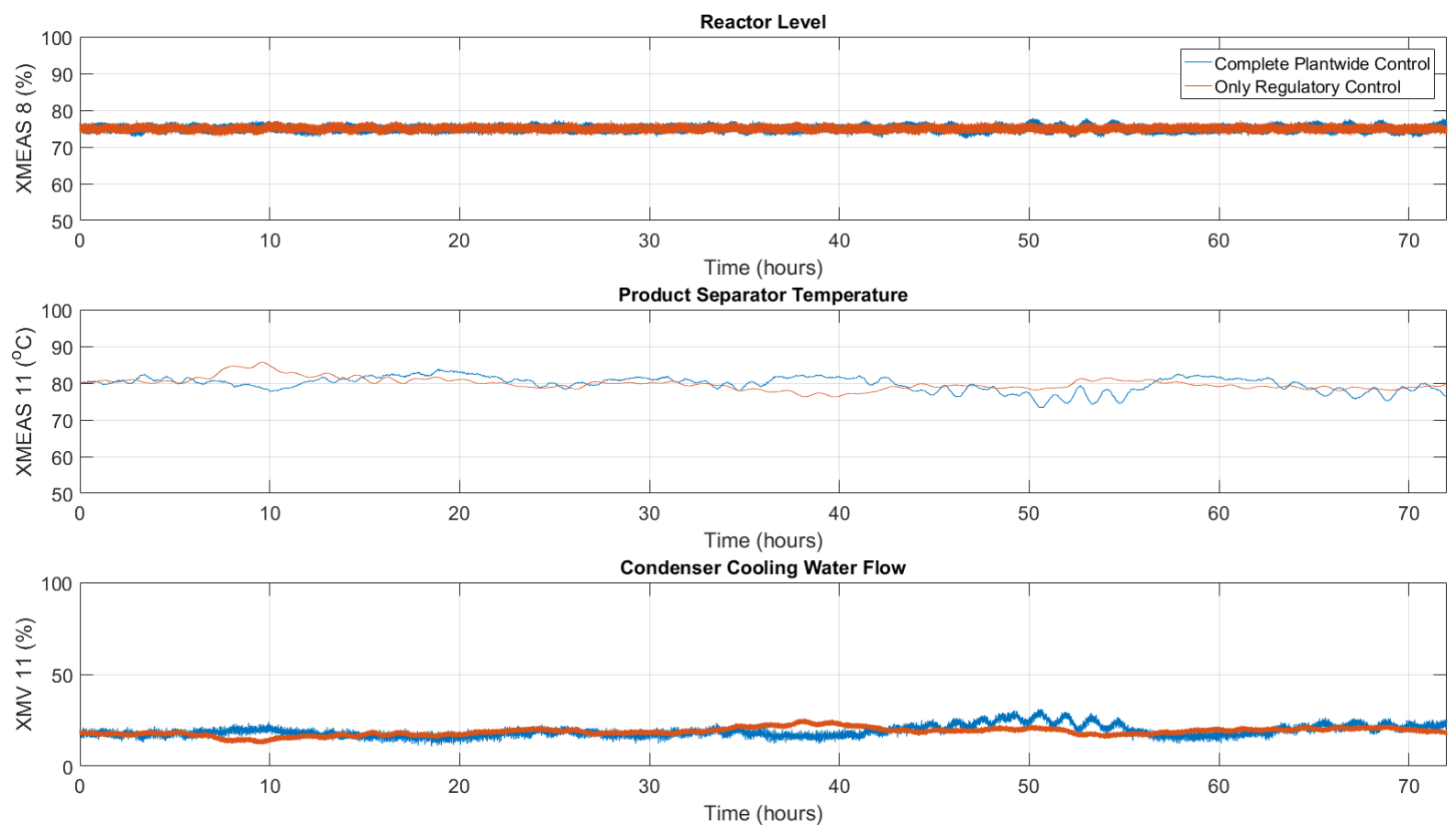

Figure 6.11 - Regulatory response for XMEAS 8 and XMEAS 11.

\subsubsection{XMEAS 9 (Reactor Temperature)}

The process response for a step change in the setpoint of XMEAS 9 from $120.4^{\circ} \mathrm{C}$ (nominal) to $122.9^{\circ} \mathrm{C}$ (Mode 1 ) is shown in Figure 6.12.

For this case, the setpoint tracking for the PID controller is faster and smoother than for the MPC controller. This happens because the MPC uses small variations of the reactor temperature inside the operating limits to regulate the reactor pressure, since this variable affects much less the operating cost than the purge rate paired with the reactor pressure. In the implemented plantwide control system, small variations in the reactor temperature are used to regulate the reactor pressure, and the purge rate is used to compensate strong disturbances, which results in a better setpoint tracking for the pressure with minor variations in the temperature.

The regulatory response for XMEAS 9 is shown in Figure 6.13, in which it can be seen that this controlled variable is kept close to its setpoint even with the PID setpoint changes set by the MPC layer to compensate pressure variations. 

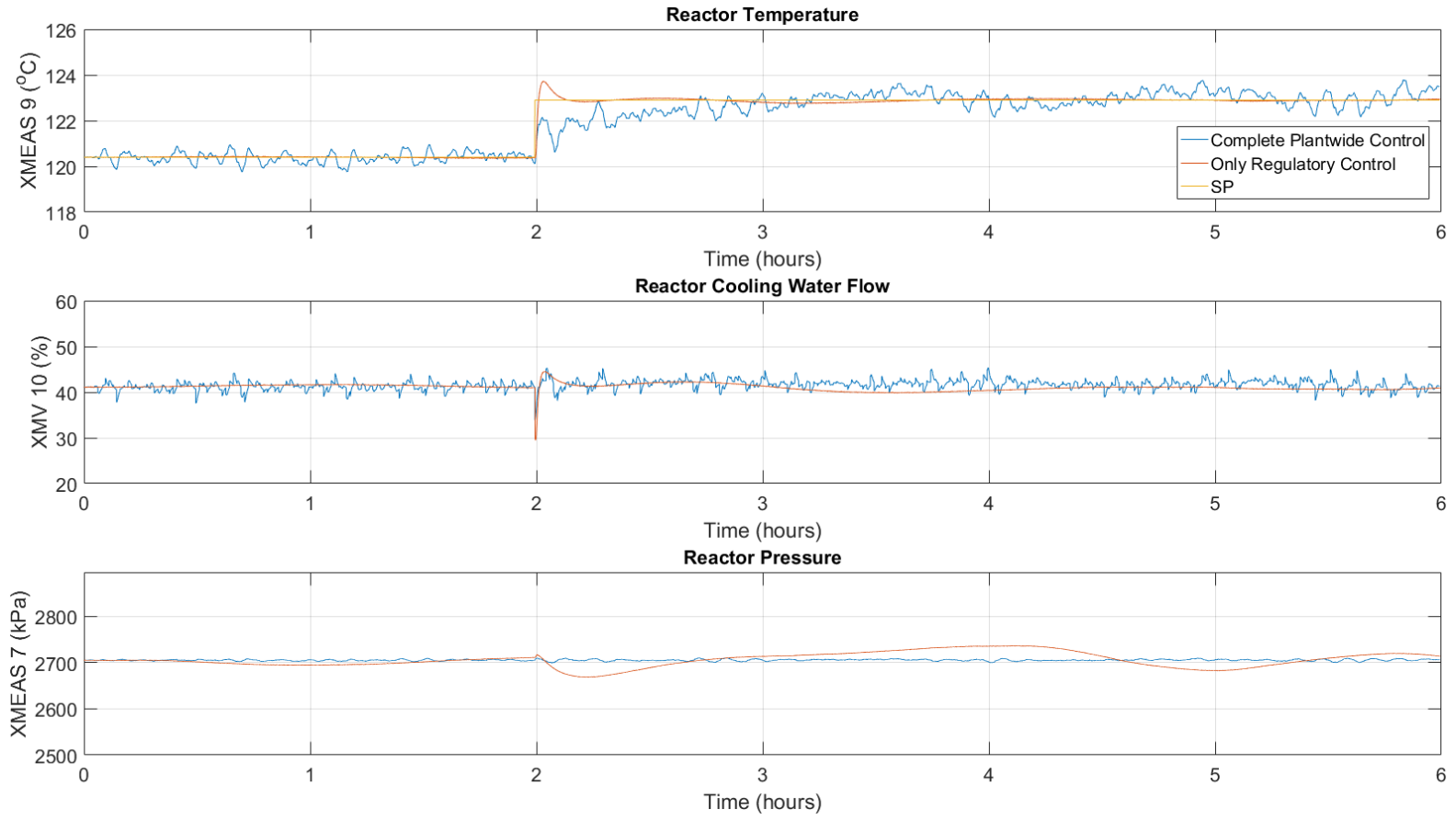

Figure 6.12 - Step response for XMEAS 9.
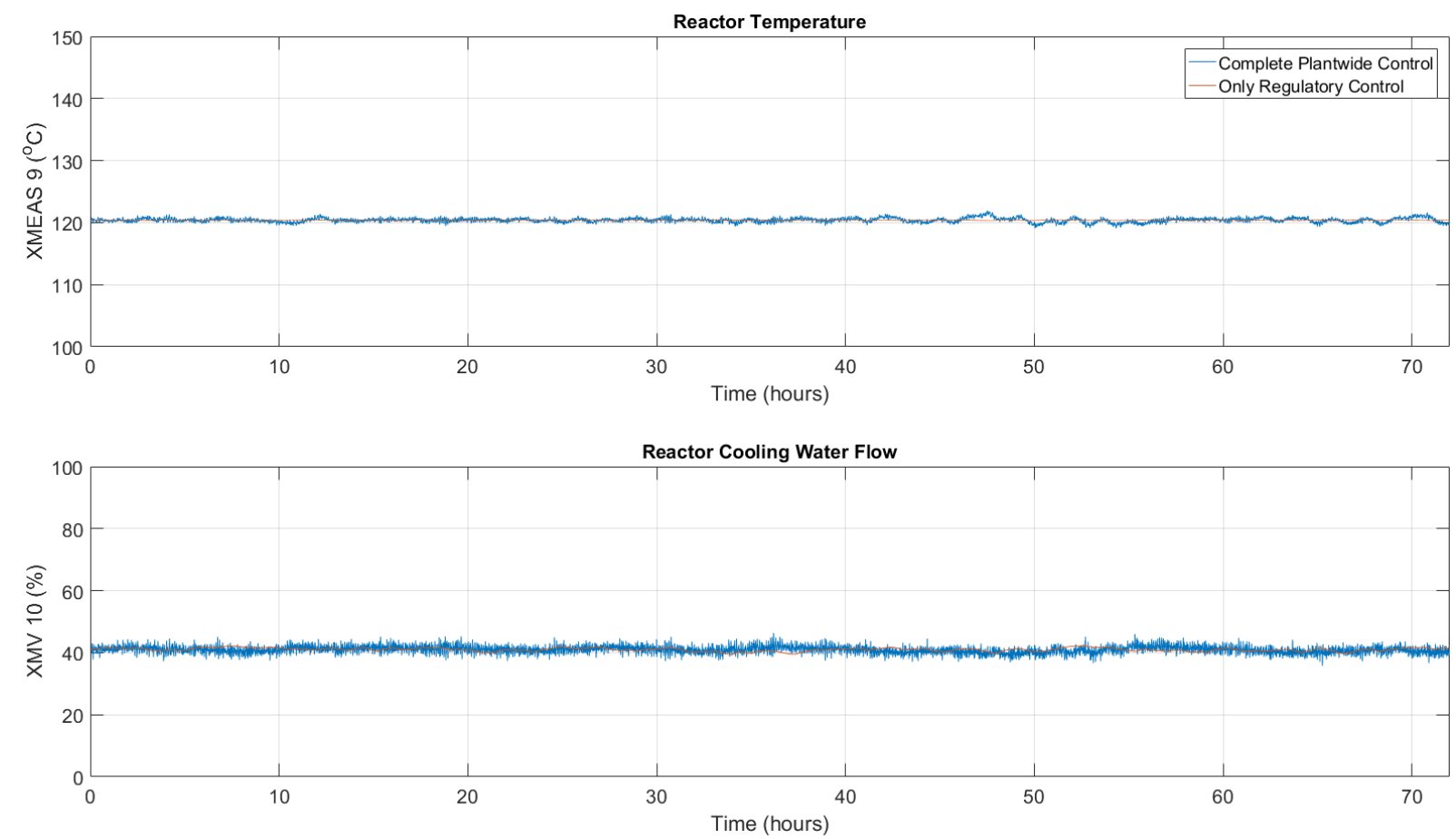

Figure 6.13 - Regulatory Response for XMEAS 9.

\subsubsection{XMEAS 12 (Product Separator Level) and XMEAS 14 (Product Separator Underflow)}

Next, Figure 6.14 shows the servo response for XMEAS 12 and Figure 6.15 shows the regulatory response for XMEAS 12 and XMEAS 14. 

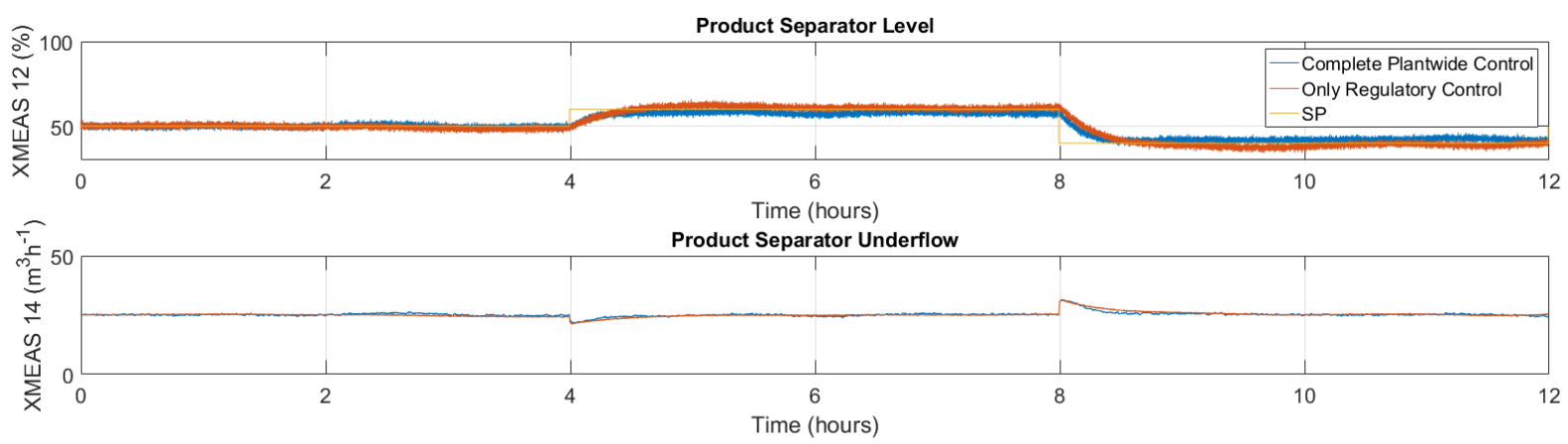

Separator pot liquid liquid flow
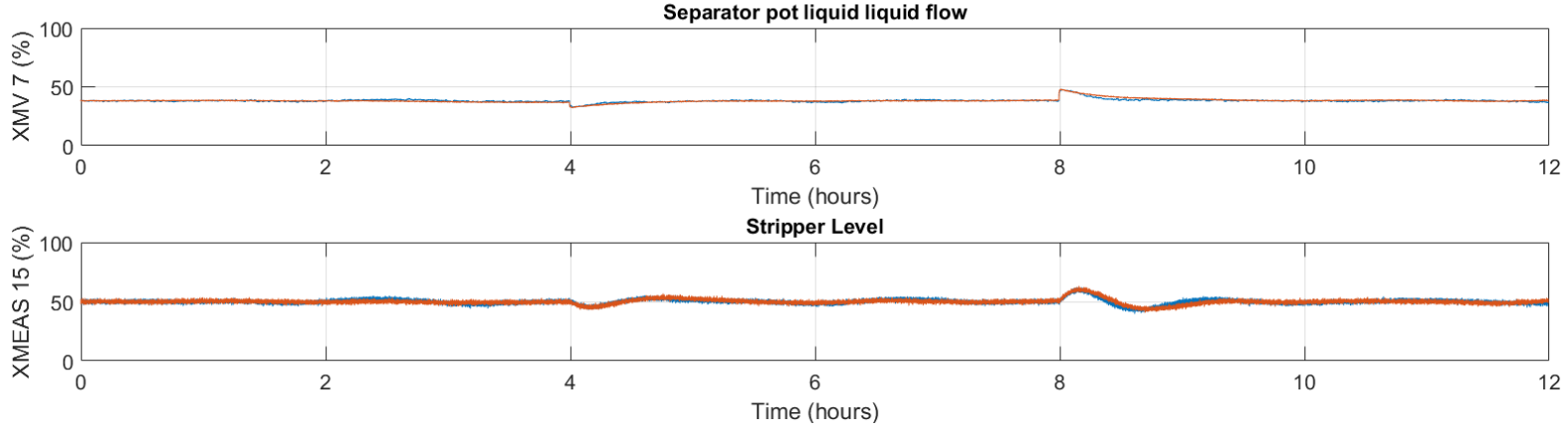

Figure 6.14 - Step response for XMEAS 12.
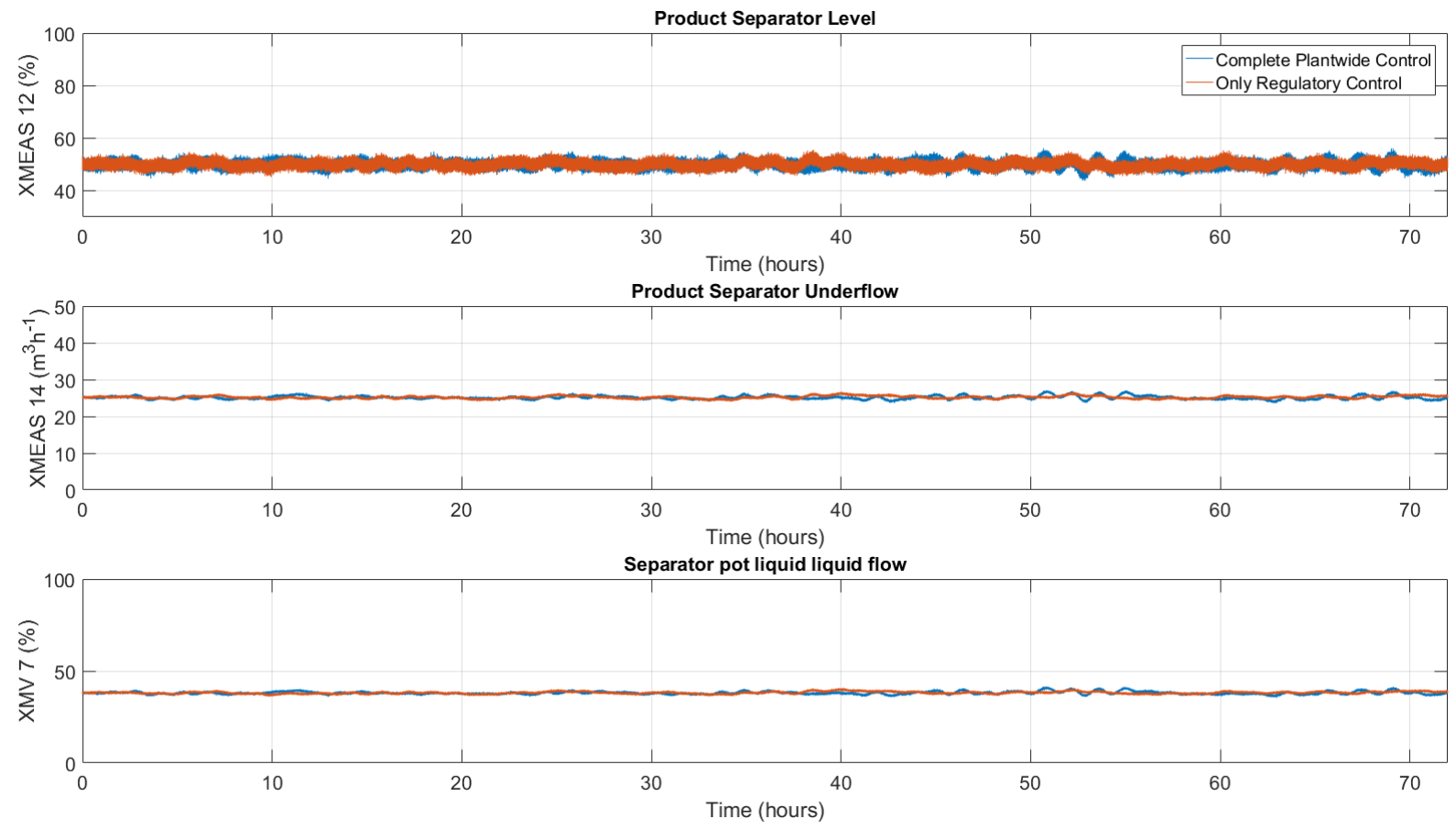

Figure 6.15 - Regulatory response for XMEAS 12 and XMEAS 14.

For these variables, both setups perform satisfactorily and present very similar responses in servo and regulatory modes. 


\subsubsection{XMEAS 15 (Stripper Level) and XMEAS 17 (Stripper Underflow)}

Next, step response for XMEAS 15 and regulatory responses for XMEAS 15 and XMEAS 17 are shown in Figure 6.16 and 6.17, respectively.
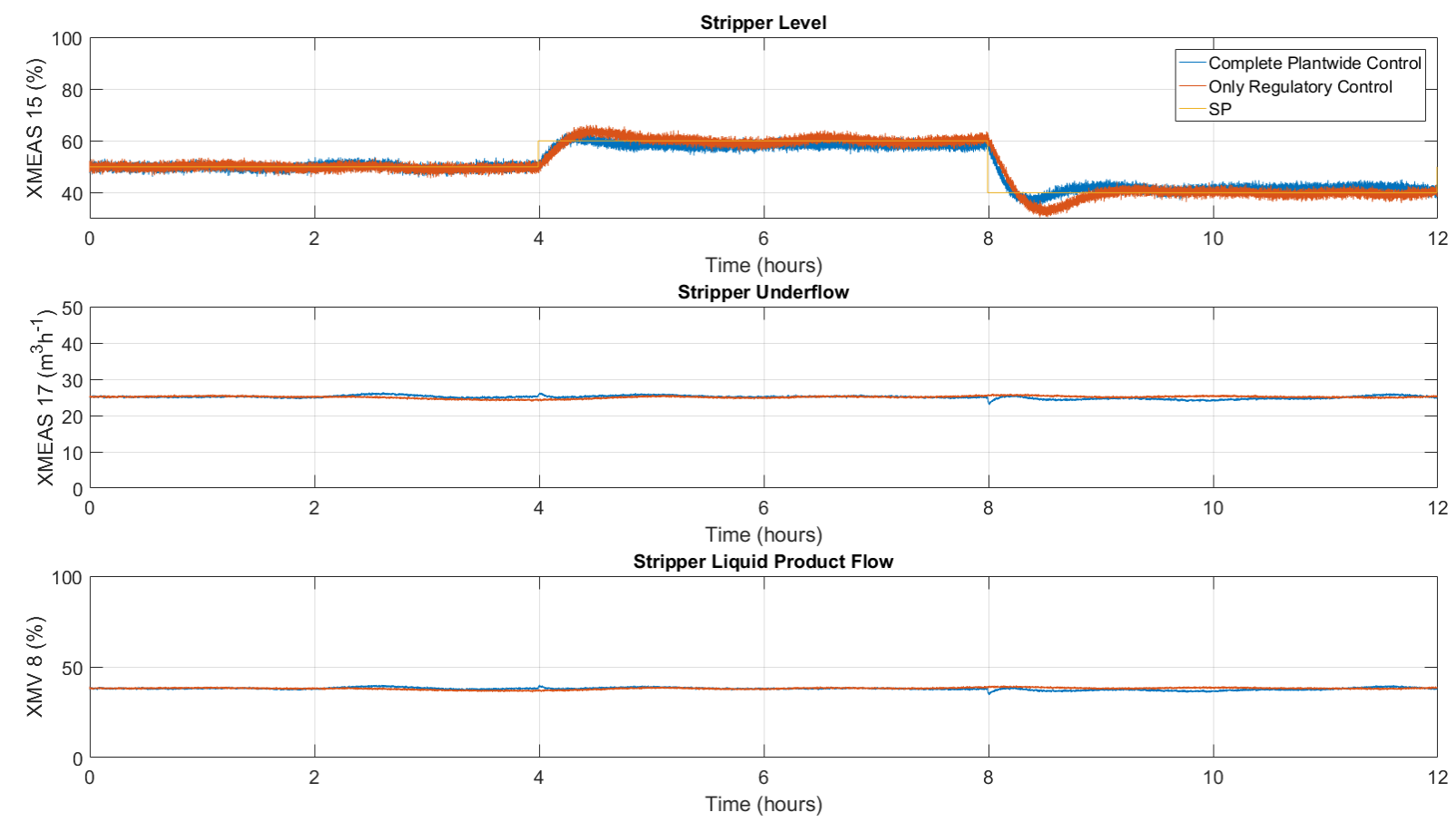

Figure 6.16 - Step response for XMEAS 15.
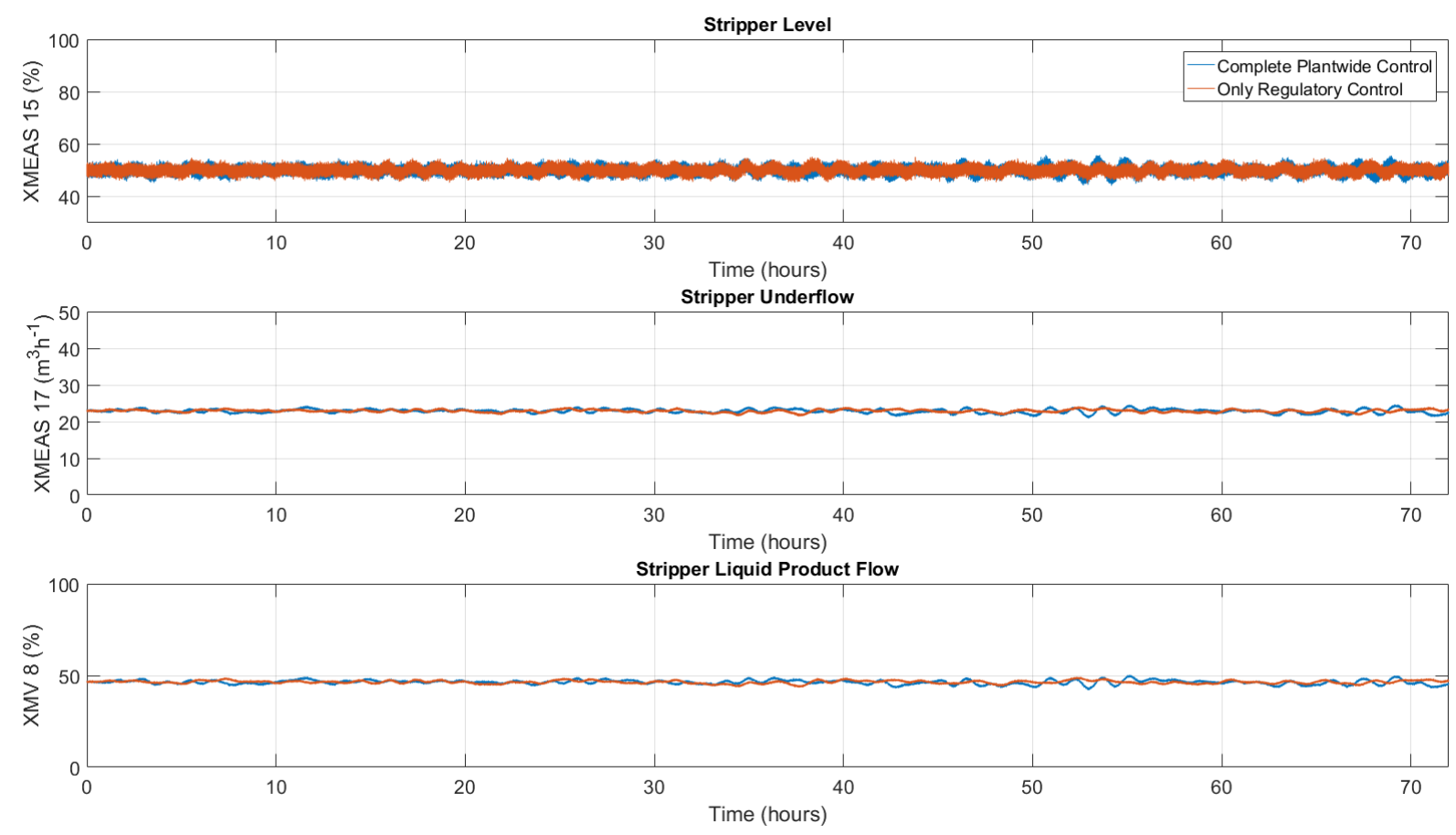

Figure 6.17 - Regulatory response for XMEAS 15 and XMEAS 17.

The control performance is good in servo and regulatory modes. 


\subsubsection{XMEAS 18 (Stripper Temperature) and XMEAS 19 (Stripper Steam Flow)}

The step response for XMEAS 18 is shown in Figure 6.18 and the regulatory responses of XMEAS 18 and XMEAS 19 for 72 hours of operation are shown in Figure 6.19.
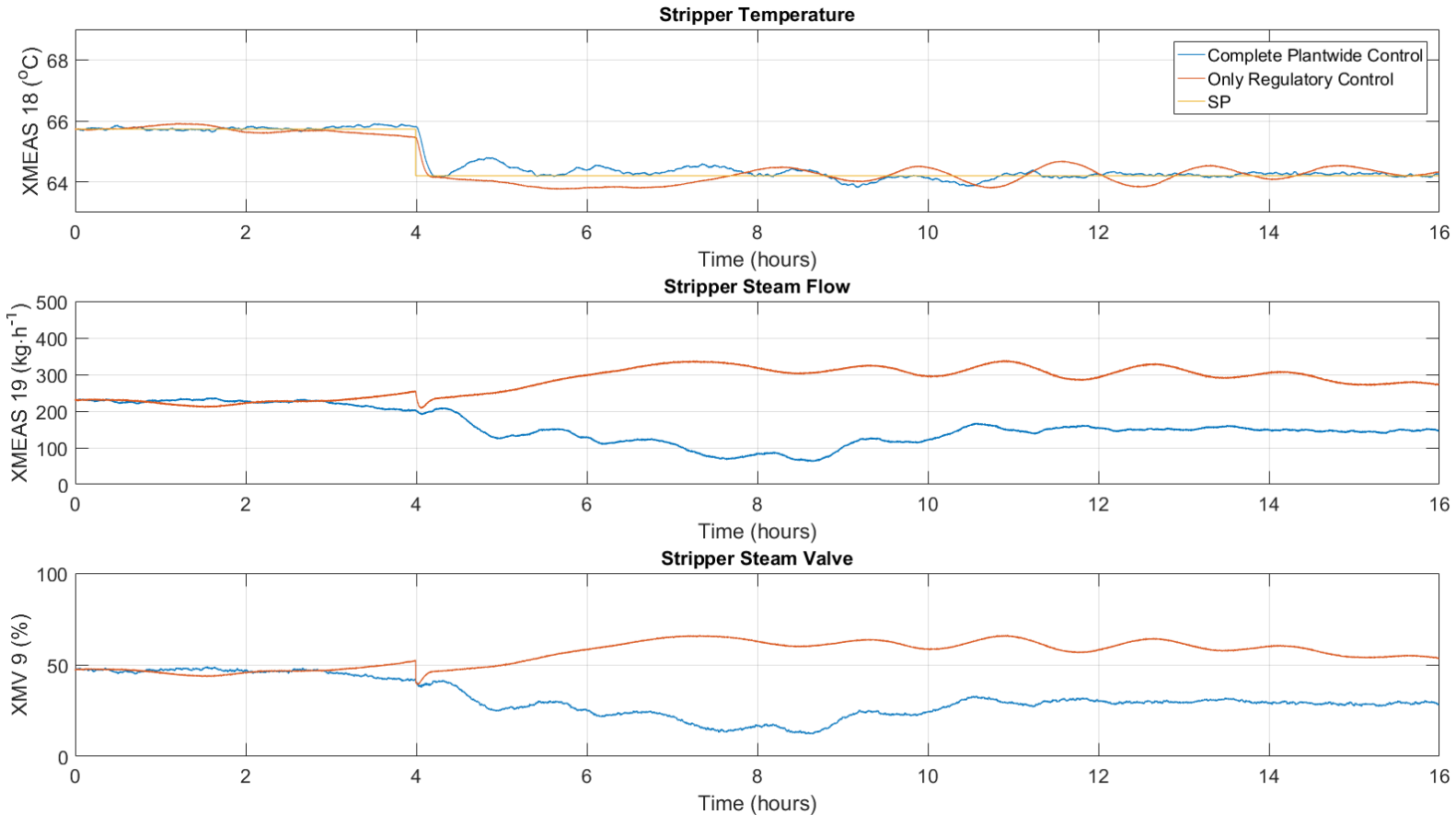

Figure 6.18 - Step response for XMEAS 18.
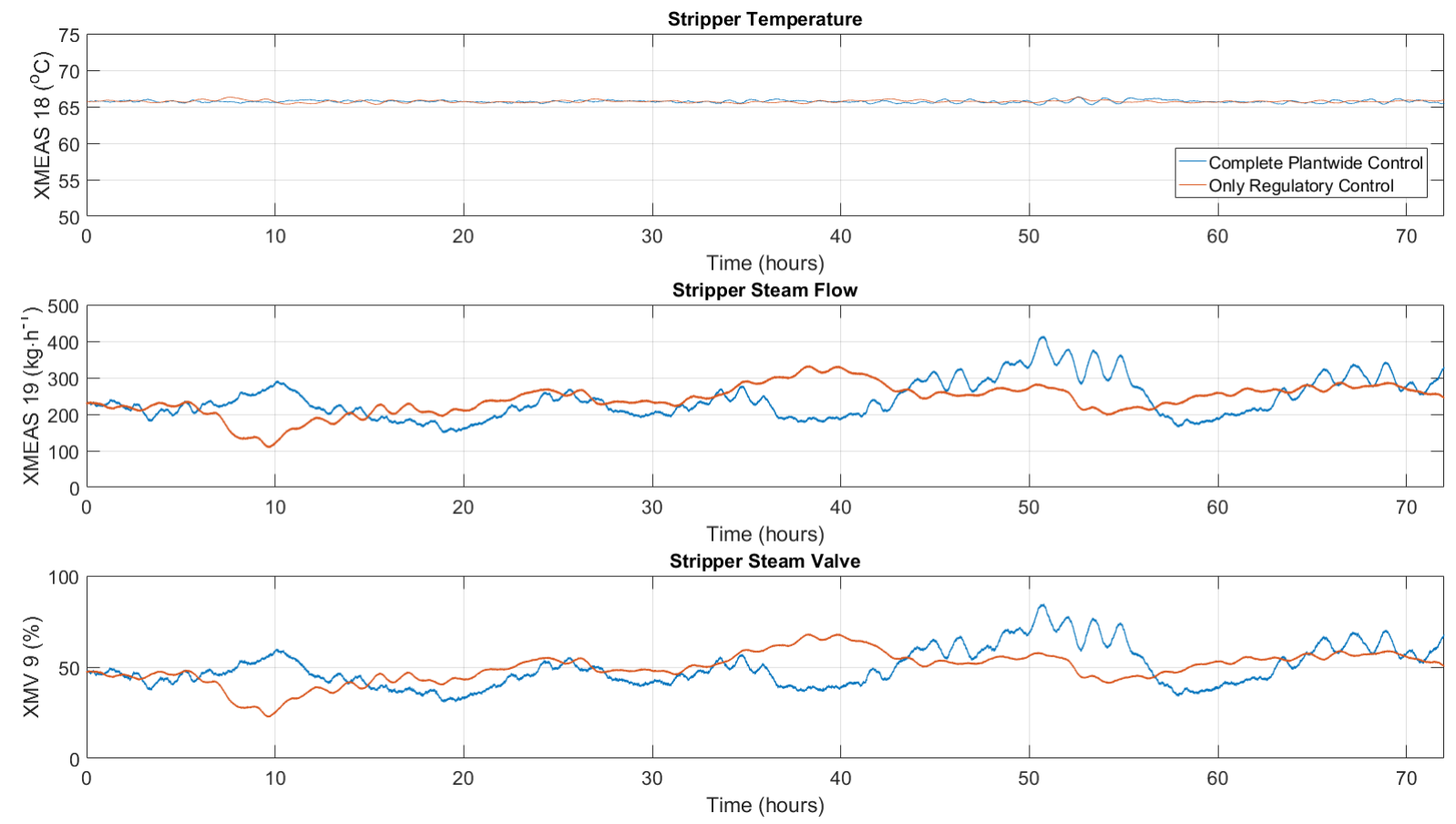

Figure 6.19 - Regulatory response for XMEAS 18 and XMEAS 19. 
The system performance is satisfactory for these variables too. It can be noted that the system with MPC control active presents a better step response, stabilizing the controlled variable at the new setpoint faster and without oscillations.

\subsubsection{XMEAS 21 (Reactor Cooling Outlet Temperature)}

The last inspected variable is XMEAS 21, with its servo mode response presented in Figure 6.20.
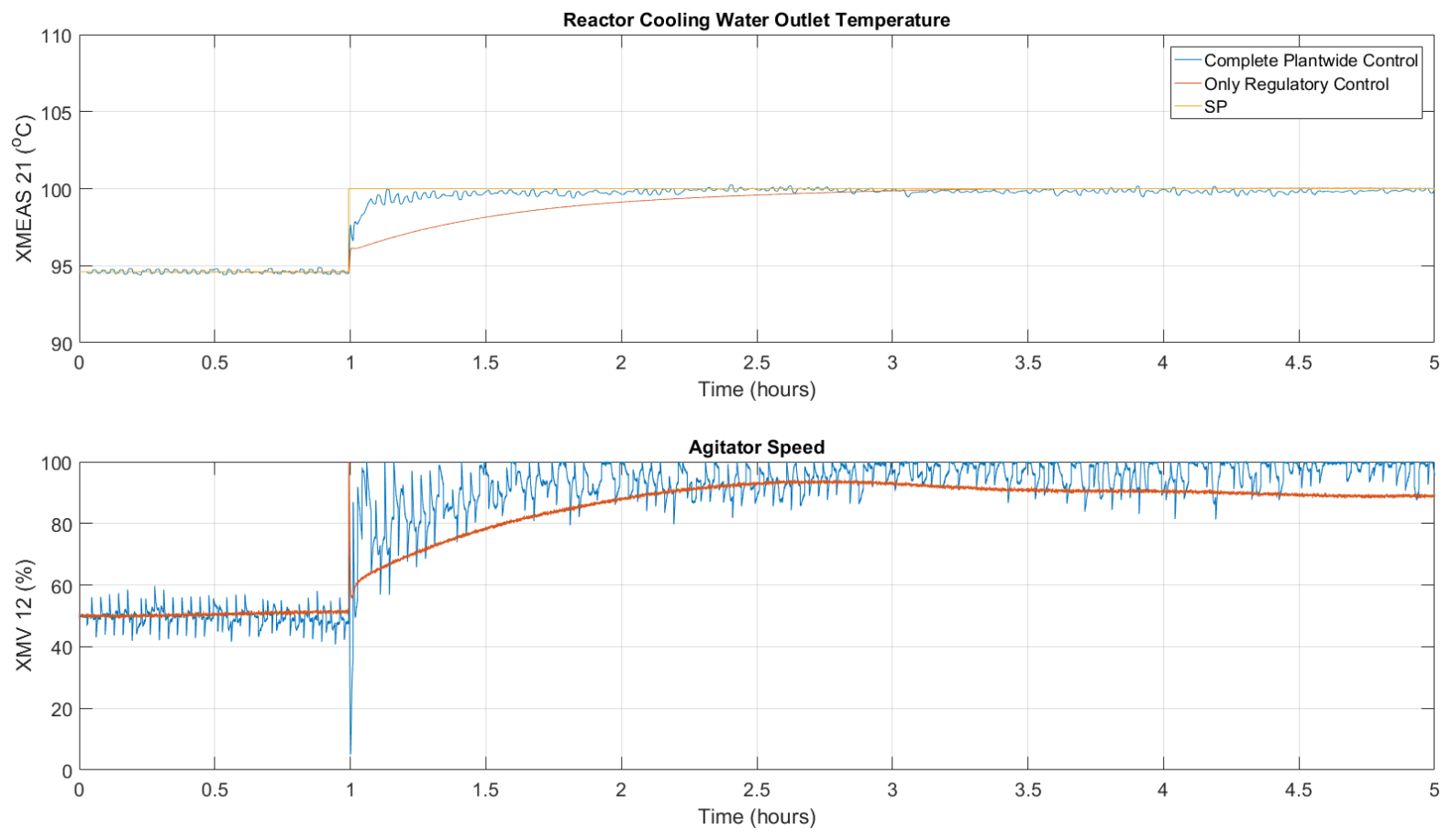

Figure 6.20 - Step response for XMEAS 21.

It can be seen that the MPC control provides a much faster response, but is significantly more oscillatory than the regulatory level control.

The regulatory response for XMEAS 21 is shown in Figure 6.21. Again it can be seen that the MPC causes an increase in oscillations when compared to the pure regulatory control. 

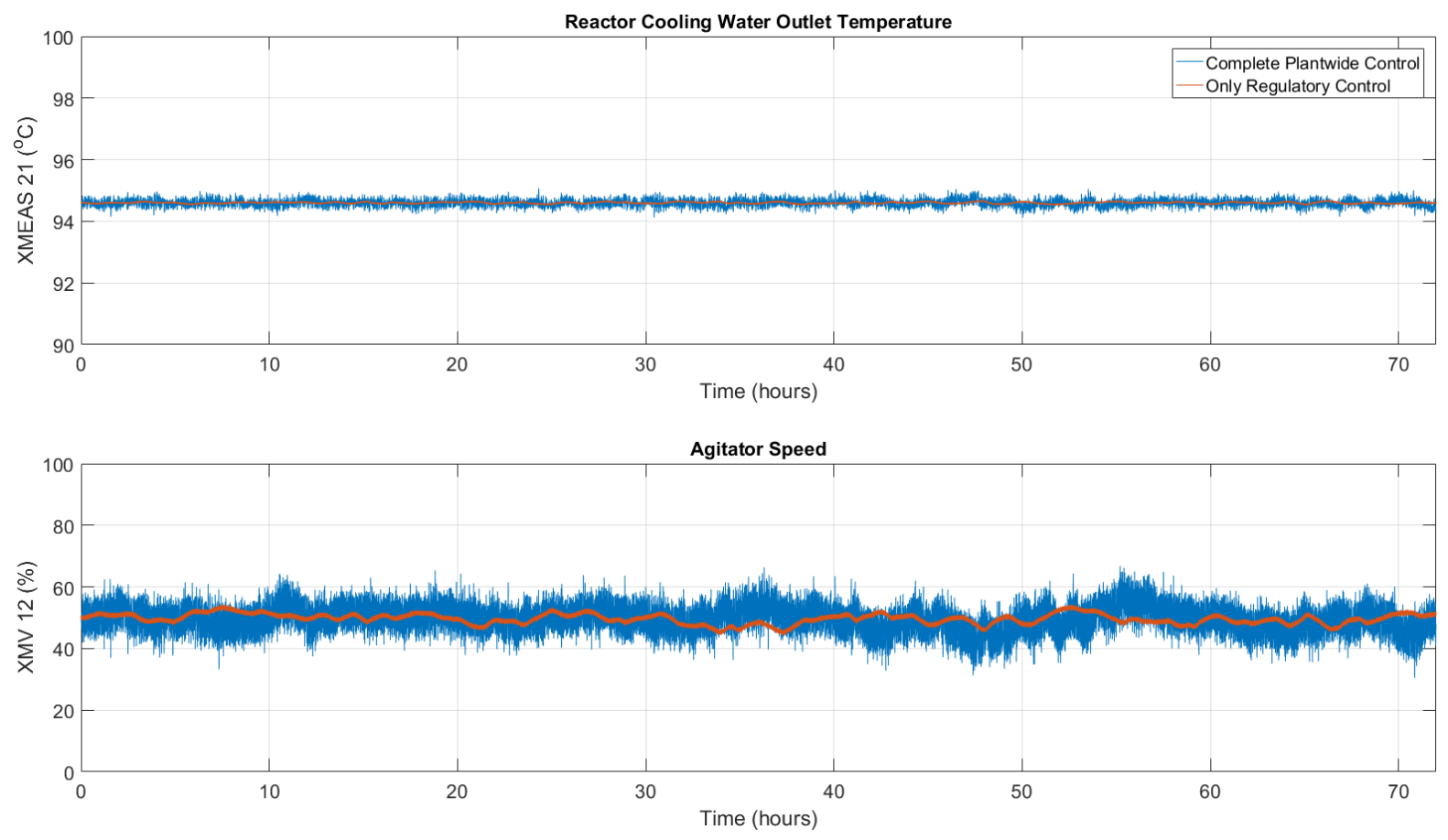

Figure 6.21 - Regulatory response for XMEAS 21.

\subsubsection{Concluding Remarks}

The final plantwide control system was considered satisfactory to control the process following all specifications.

Some controlled variables lost some control quality when the MPC layer was added, which is due to the fact that the controller used such variables to aid on the control of others with higher priority. It was considered that the MPC control provided an overall quality gain, improving the process stability and response times.

\subsection{Operating Tests}

To further evaluate the control system and demonstrate its operability, the plant is setup to operate at three different operating points: nominal (used in the design), Mode 1 and Mode 3; all three following the specifications detailed by (Bathelt, et al., 2015). The setpoints used for each variable are shown in Table 6.14. 
Table 6.14 - Setpoints for the three tested scenarios.

\begin{tabular}{|c|c|c|c|}
\hline \multirow{2}{*}{ Controlled Variable } & \multicolumn{3}{|c|}{ Operating Mode } \\
\hline & Nominal & Mode 1 & Mode 3 \\
\hline$\overline{X M E A S ~} 1$ & 0.25052 & 0.2710 & 0.195 \\
\hline XMEAS 2 & 3664.0 & 3664.0 & 5180 \\
\hline XMEAS 3 & 4509.3 & 4450 & 720.071 \\
\hline XMEAS 4 & 9.3477 & 9.22 & 7.83 \\
\hline XMEAS 5 & 26.902 & 38 & 20.0 \\
\hline XMEAS 6 & 42.339 & 52.5 & 32.16 \\
\hline XMEAS 7 & 2705 & 2800 & 2800 \\
\hline XMEAS 8 & 75 & 65 & 65 \\
\hline XMEAS 9 & 120.44 & 122.9 & 121.9 \\
\hline XMEAS 10 & 0.33712 & 0.22 & 0.08045 \\
\hline XMEAS 11 & 80.109 & 95 & 99 \\
\hline XMEAS 12 & 50 & 50 & 50 \\
\hline XMEAS 13 & 2633.7 & 2718 & 2763 \\
\hline XMEAS 14 & 25.160 & 25.160 & 17.57 \\
\hline XMEAS 15 & 50 & 50 & 50 \\
\hline XMEAS 16 & 3102.2 & 3340 & 2995 \\
\hline XMEAS 17 & 22.949 & 22.89 & 18.04 \\
\hline XMEAS 18 & 65.731 & 66.7 & 62.368 \\
\hline XMEAS 19 & 230.31 & 0 & 5.33 \\
\hline XMEAS 20 & 341.43 & 318 & 273.6 \\
\hline XMEAS 21 & 94.599 & 100 & 101.88 \\
\hline XMEAS 22 & 77.297 & 95 & 45.57 \\
\hline
\end{tabular}

The process and controllers' setup (initial conditions and nominal values) are updated for each simulated condition, and the final tuning refinement is repeated for each operating point.

The MPC weights updated for Mode 1 are:

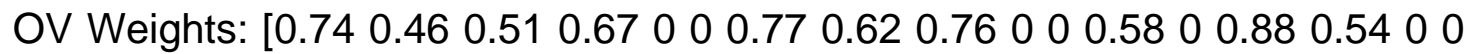
$0.88000 .530]$; 
$>\quad$ MV Weights: $[0.20 .400 .100 .30110 .70 .10]$;

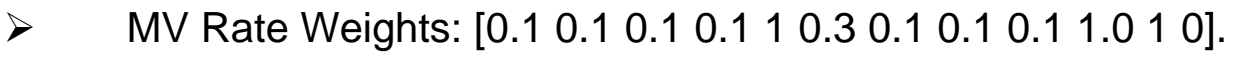

The MPC weights updated for Mode 3 are:

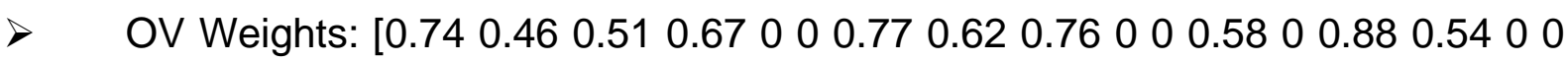
$0.88000 .530] ;$

> MV Weights: [0.7 0.200 .800 .901100 .10$]$;

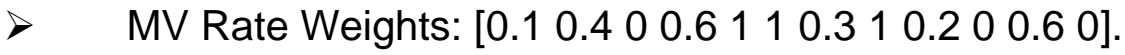

The process operation is tested in the three scenarios.

Figure 6.22 shows the process variables and Figure 6.23 shows the manipulated variables for the system in nominal operating point for 72 hours.
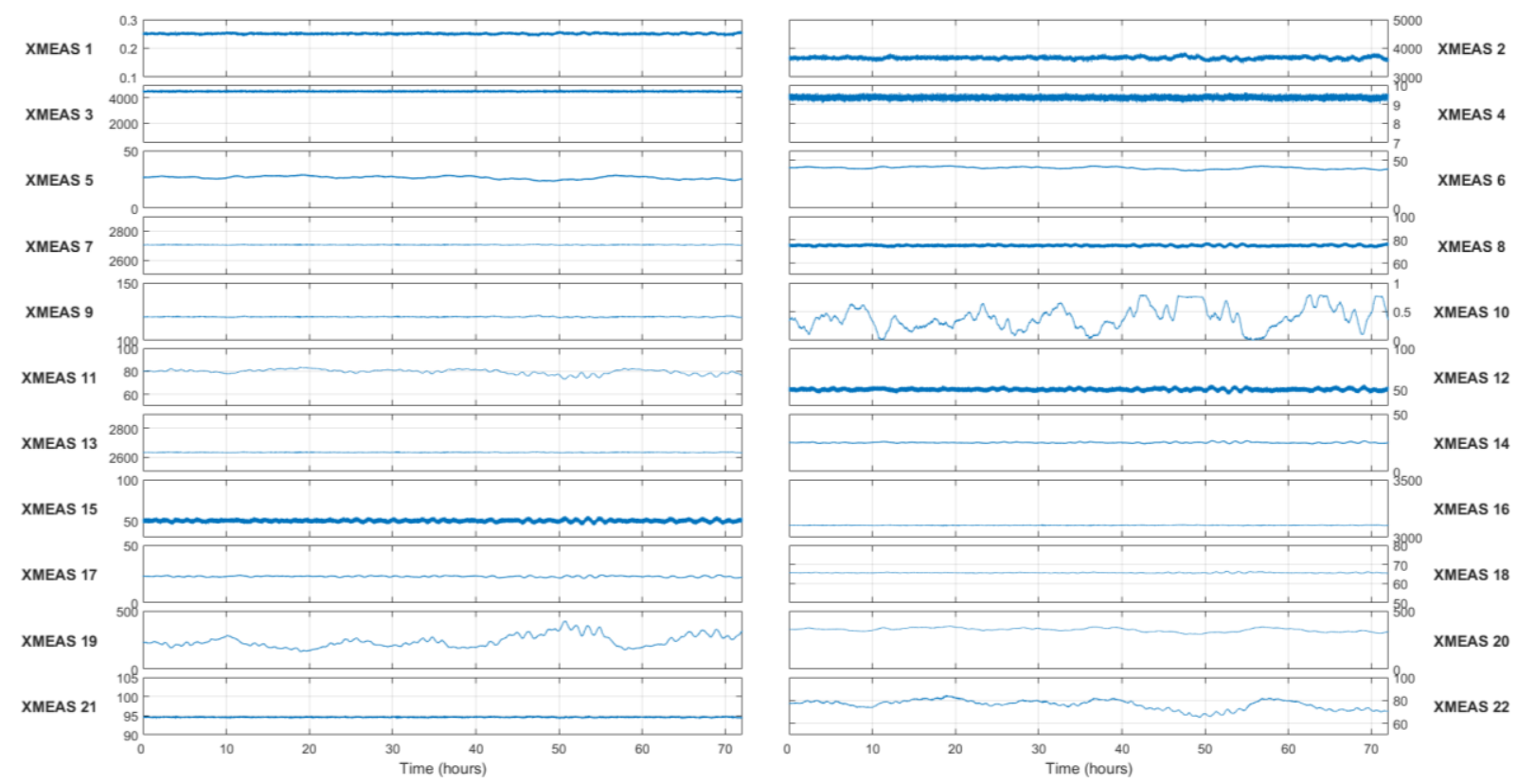

Figure 6.22 - Process variables for the Tennessee Eastman in nominal operation. 

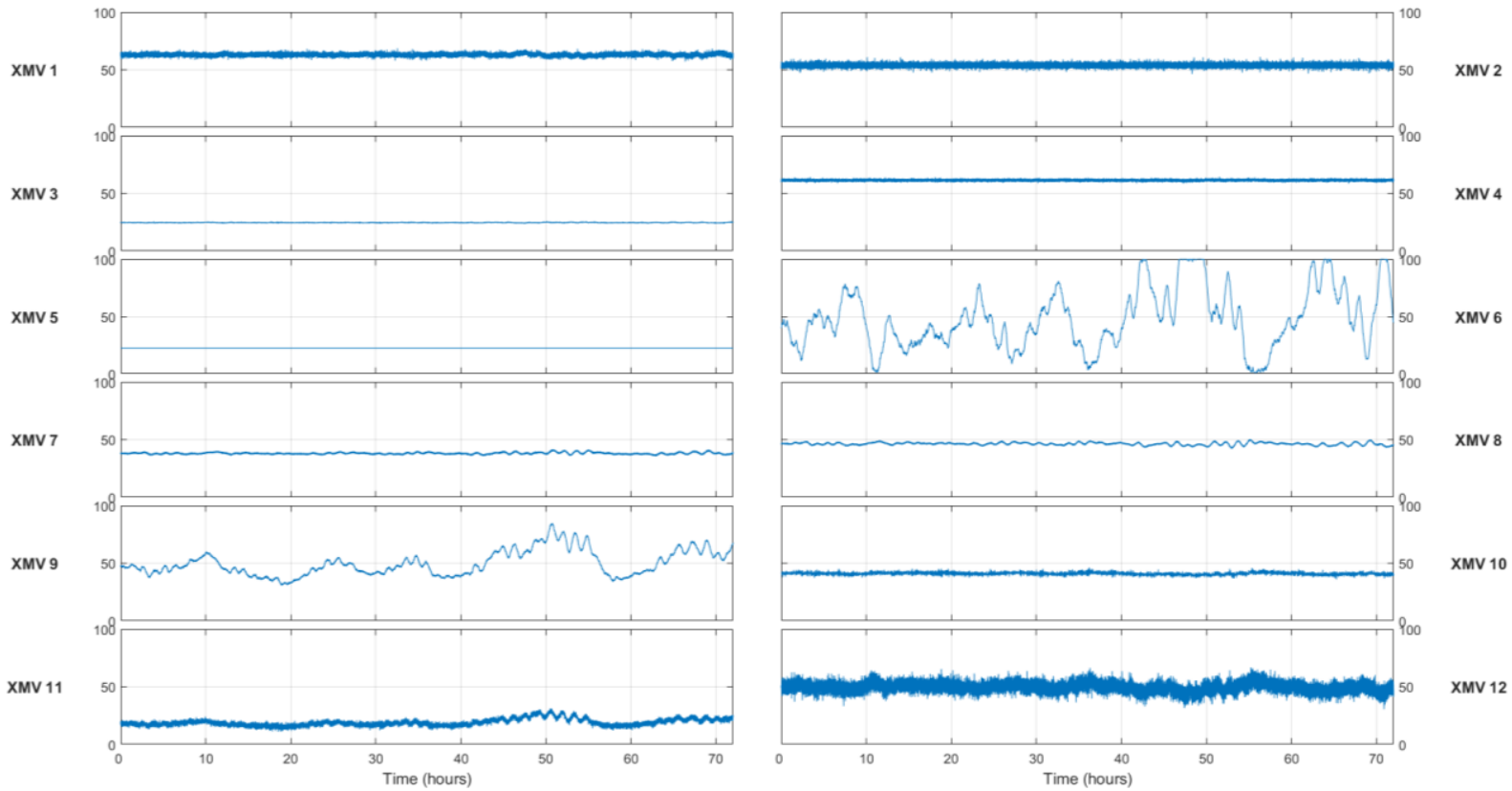

Figure 6.23 - Manipulated variables for the Tennessee Eastman in nominal operation.

Next, Figures 6.24 and 6.25 show the process and manipulated variables for the system operating with Mode 1 setpoints for 72 hours.
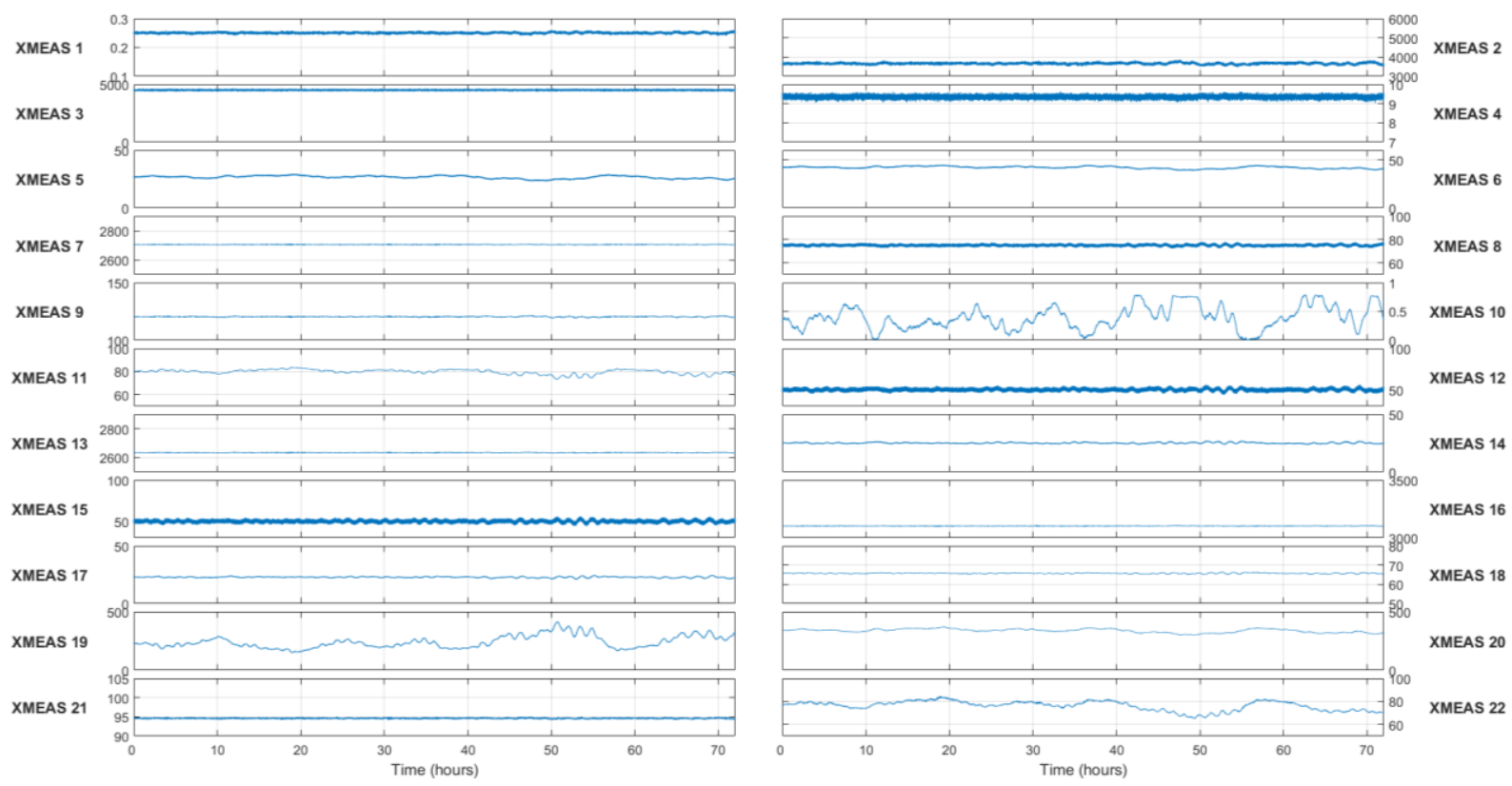

Figure 6.24 - Process variables for the Tennessee Eastman in mode 1 operation. 

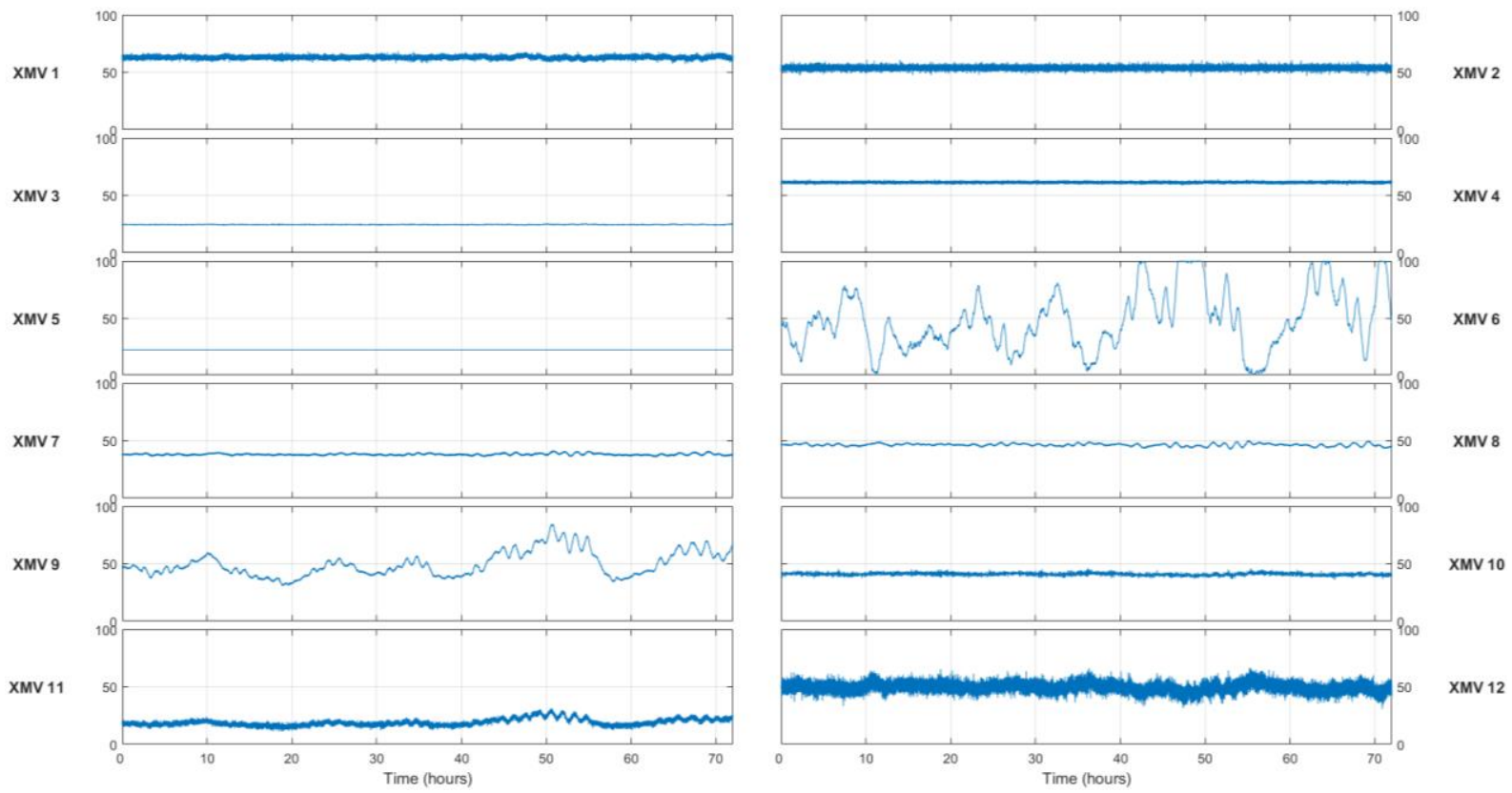

Figure 6.25 - Manipulated variables for the Tennessee Eastman in mode 1 operation.

Next, Figures 6.26 and 6.27 show the process and manipulated variables for the system operating with Mode 3 setpoints for 72 hours.
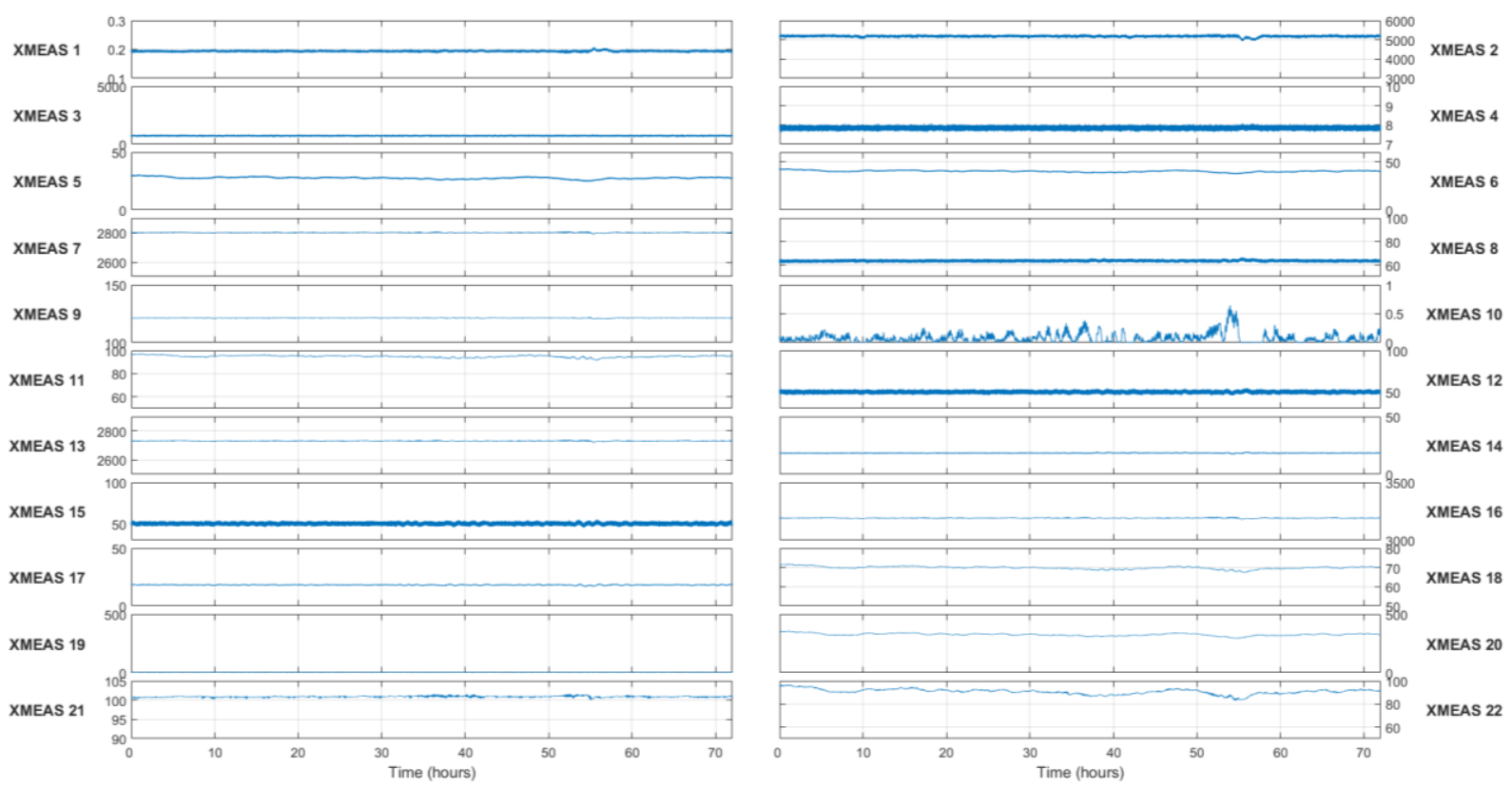

Figure 6.26 - Process variables for the Tennessee Eastman in mode 3 operation. 

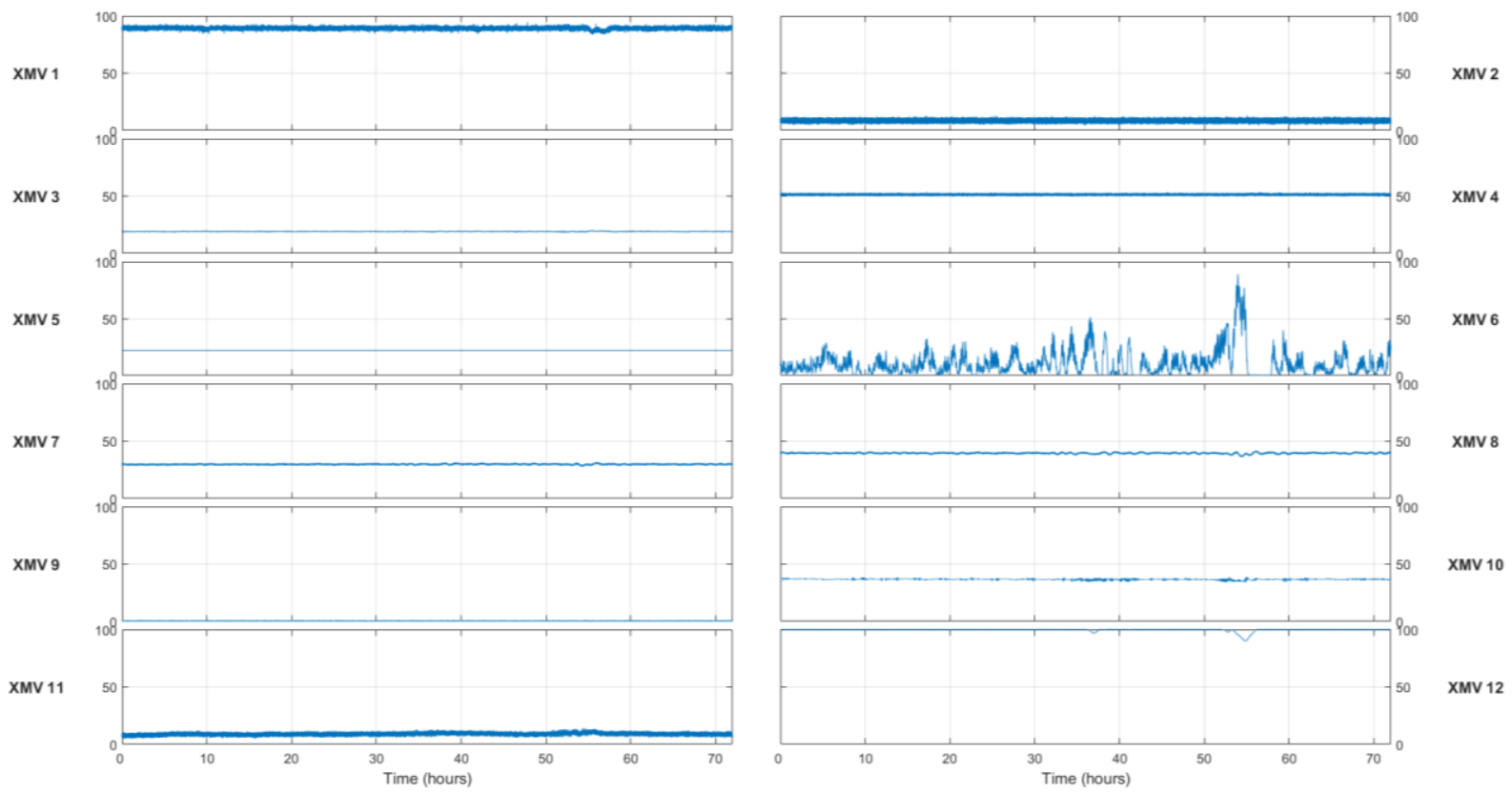

Figure 6.27 - Manipulated variables for the Tennessee Eastman in mode 3 operation.

Finally, the operating costs for the process in the three tested scenarios are shown in Figure 6.28.

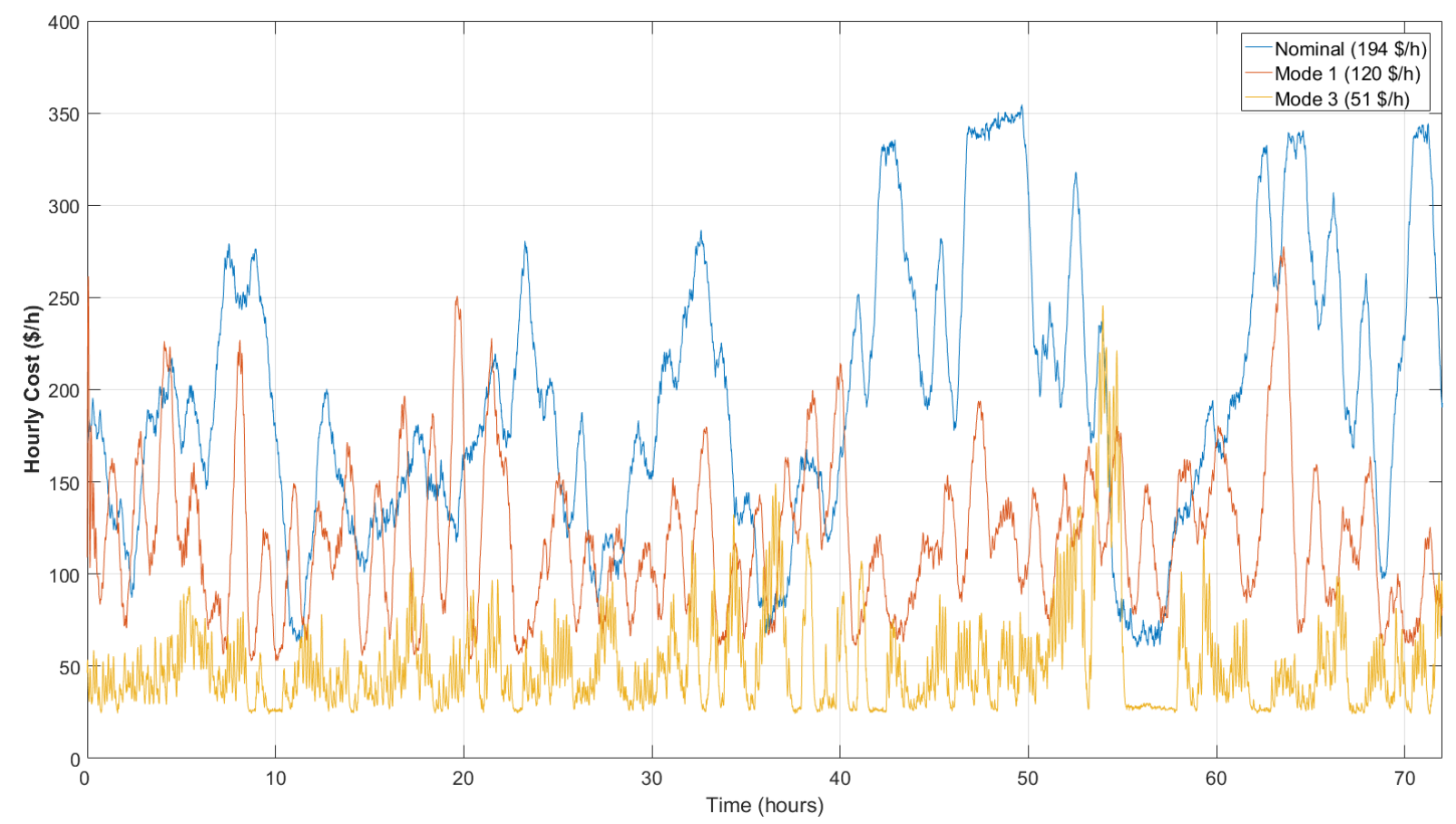

Figure 6.28 - Operating costs for the Tennessee Eastman Challenge Process.

For a quantitative comparison, Table 6.15 presents the average operating cost observed in each case, with results presented for the complete control system active, for only PID controllers active and also using the control system given in (Bathelt, et al., 2015) for comparison. 
Table 6.15 - Average operating cost for $72 \mathrm{~h}$ of operation in three operating modes.

\begin{tabular}{c|c|c|c}
\hline \multirow{2}{*}{ Tested Scenario } & \multicolumn{3}{|c}{ Operating Mode } \\
& Nominal & Mode 1 & Mode 3 \\
\hline MPC control active & $194 \$ / \mathrm{h}$ & $120 \$ / \mathrm{h}$ & $51 \$ / \mathrm{h}$ \\
\hline Only PID controllers active & $184 \$ / \mathrm{h}$ & $120 \$ / \mathrm{h}$ & $58 \$ / \mathrm{h}$ \\
\hline Reference (Bathelt, et al., 2015) & - & $118 \$ / \mathrm{h}$ & $146 \$ / \mathrm{h}$ \\
\hline
\end{tabular}

For the nominal operating point, the MPC layer increases the operating cost by $5 \%$ to better regulate the process variables, when compared to the designed regulatory layer, as seen in the previous section. At Mode 1 operation the control quality gain does not cause any increase in the operating cost, and the designed system presents a similar cost to the reference control system. At Mode 3 operation, the designed system is able to operate at an hourly cost significantly lower than the reference control system, and the complete system presents a cost reduction of $12 \%$ when compared to the pure regulatory control.

The control design was considered successful, and the final control system was successfully validated in three different operating points.

\subsection{Concluding Remarks}

A plantwide control system was designed to the Tennessee Eastman Challenge process. The designed system managed to successfully control the plant and follow the desired specifications.

The design resulted in a classical control architecture with regulatory PID control layer and a supervisory MPC control layer, that also performs the plantwide economic optimization of the process.

The system was tested and evaluated in three different operating modes, operating satisfactorily in all of them. All the design steps and results were detailed along the presented description.

The new design procedure integrated system identification and control tuning in the plantwide design steps. With this, no assumptions about the process dynamics or 
about perfect control were made, in contrast to most plantwide design procedures. The new procedure allowed the identification experiments to be better designed, with focus given to the most important dynamic relations, which resulted in better identified models. With the control tuning also performed during the design procedure instead of after all decisions had been made, such decisions could be better done, by consideration of the real control performance instead of assuming a perfect control, in contrast to what it is usually done in other approaches.

The design results were considered successful and the adopted techniques and tools were considered valuable and could be potentially used in practical applications. 


\section{Conclusions}

The plantwide control design problem was studied and some contributions were proposed in this thesis.

Initially, plantwide control was briefly described, with a focus on the concept and its relevance to both industrial applications and academic development. Next, a broad review of the topic was presented, describing plantwide control in detail and highlighting the major available techniques, the most relevant previous works and open research topics.

While plantwide control has been studied for some decades, starting with the publication of (Buckley, 1964) and regardless of its importance in industrial application, publications concerned with the design of control systems for complete plants, instead of only a simple unit, have appeared only in the last 15 years, making it a new area and with many opportunities for development and innovations.

Even though the number of publications in the subject has been increasing over the years, consolidated literature is scarce, as well as formalized courses, which highlights the relevance of the presented review as an investigation of the current state of the art of plantwide control.

To build a new plantwide control design technique with relevant novelties, some theoretical tools were presented, with the addressing of system identification for large scale systems, definition of optimality, description of Bellman's principle of optimality in the plantwide control context, description of the Nash equilibrium and Pareto optimality concepts and its relevance to plantwide control, discussion of cooperation between multiple controllers acting on the same system, presentation of an optimal control tuning technique and proposal of an augmented multi-objective optimization procedure.

Among the presented tools, the proposed Augmented Lexicographic MultiObjective Procedure allows an efficient optimization of multiple conflicting objectives to achieve a true multi-objective optimal solution with good control of trade-offs.

A novel Augmented Hierarchical Plantwide Control Design Technique was proposed, applying the discussed concepts and the presented tools. The proposed 
technique has the following highlights: inclusion of system identification in the plantwide design procedure to enhance the quality of the identification results and reduce the experimental costs; inclusion of control tuning as part of the design procedure to allow better decisions to be taken and that the final implemented control system behaves close to the designed system; applicability to large systems, to different types of processes and controller; support to multi-objective specifications; and design of a plantwide robust optimal control system.

An Optimal Multi-Objective Technique for Integrated Control Structure Selection and Controller Tuning was also proposed. This technique deals with the problems of I/O pairings and optimal control tuning, solving both problems simultaneously.

Tests with five industrial benchmarks were performed to investigate the relationship between I/O pairing and control tuning. It was verified that these are two highly intertwined problems that should be solved together, and not separately as it is usually done. These tests were the basis for the proposal of the mentioned Optimal Multi-Objective Technique for Integrated Control Structure Selection and Controller Tuning.

To evaluate the proposed techniques, plantwide control systems were designed for two industrial benchmarks, a heavy oil fractionator unit with 3 manipulated and 7 controlled variables and a fluid catalytic cracking unit with 6 manipulated and 7 controlled variables. The results showed that the proposed approaches could be successfully used to build control systems with well accepted architectures.

To further evaluate and improve the proposed techniques, a final application example was performed with the Tennessee Eastman Challenge Process, a complex nonlinear system with 12 manipulated and 22 controlled variables. A plantwide control system was built for this process, with the final system tested and validated in three operating points. This example included all the plantwide control design steps and was presented to show a detailed description of the application of the proposed techniques.

The control systems designed in all examples resulted in a classical and well accepted control architecture, with the novelties of proposed procedures residing in how to design such control system, i.e., the proposals provided new and better ways 
to build and tune widely tested controllers. This characteristic further enhances the applicability of the proposed methods to real industrial plants in the near future.

All the objectives stablished for this work were accomplished, as well as the goal of proposing a new plantwide control design technique. The proposed objective O-1, "review of Plantwide Control", resulted in the review presented in Chapter 2. Objective O-2, "description and solution of the problem of multi-objective optimization", resulted in the concepts summarized in Section 3.6 and the proposal of the Augmented Lexicographic Multi-Objective Optimization Procedure for choosing a solution from the Pareto set. Objective O-3, "formulation of the requisites of a plantwide control technique" resulted in the tools described along Chapter 3 and the technique specification presented in Section 4.1. Objective O-4, "proposal of a novel plantwide control technique" was achieved through the proposal presented in Section 4.2, and also resulted in the complementary technique proposed in Section 4.3 to deal with the I/O pairing and control tuning problems. Objective O-5, "application of the proposed approach to an industrial benchmark", was expanded to the eight case studies presented in Chapter 5 and in Chapter 6, which were crucial to the development and evaluation to the proposals of this thesis. Finally, the main goal of this thesis was achieved through the proposal of the presented Augmented Hierarchical Plantwide Control Design Technique.

Future work can address the problem of designing a plantwide control system to a nonlinear process with multiple operating points and the problem of addressing fault tolerance in the plantwide control design.

As a final contribution of this thesis, it is proposed that, as shown along this work, the concept of Plantwide Control Design can be extended, beyond being only the structural decisions of a control system, to the following:

"Plantwide Control Design is the problem of designing control systems for entire plants. It includes the tasks of process modeling and/or system identification, structural decisions and design and tuning of all the controllers that compose the control system. Such tasks are all related and should always be solved in an integrated manner for best results." 


\section{References}

Abdel-Mooty, M. \& Roorda, J., 1991. Optimal control configuration for active control of structural vibrations. USA, pp. 761-765.

Aguirre, L. A., 2007. Introdução à Identificação de Sistemas: Técnicas Lineares e NãoLineares Aplicadas a Sistemas Reais. 3rd ed. Belo Horizonte (Minas Gerais): Editora UFMG.

Al-Arfaj, M. A. \& Luyben, W. L., 2004. Plant-Wide Control for TAME Production Using Reactive Distillation. AIChE Journal, Volume 50, pp. 1462-1473.

Al-Sulaiman, F. \& Zaman, S., 1994. Actuactor placement in lumped parameter systems subjected to disturbance. Computers \& Structures, July, Volume 52, Issue 1, pp. 4147.

Alves, V. A. O., Juliani, R. C. G. \& Garcia, C., 2012a. Searching the Optimal Order for High Order Models SISO Case. In: Proceedings of the 2012 IEEE Multi-Conference on Systems and Control, MSC 2012, Dubrovnik. 2012.

Alves, V. A. O., Juliani, R. C. G. \& Garcia, C., 2012b. Searching the Optimal Order for High Order Models MIMO Case. In: Proceedings of the 2012 IEEE Multi-Conference on Systems and Control, MSC 2012, Dubrovnik. 2012.

Alves, V. A. O., Juliani, R. C. G. \& Garcia, C., 2013. Optimal Time Delay Estimation for System Identification. In: Proceedings of the 2013 American Control Conference, ACC 2013, Washington DC, USA. 2013. pp. 95-100.

Antelo, L. T., Banga, J. R. \& Alonso, A. A., 2008. Hierarchical design of decentralized control structures for the Tennessee Eastman Process. Computers \& Chemical Engineering, Volume 32, pp. 1995-2015.

Araujo, A. C. B., Govatsmark, M. \& Skogestad, S., 2007a. Application of Plant-Wide Control to the HDA Process. I - Steady-State Optimization and Self-Optimizing Control. Control Engineering Practice, Volume 15, pp. 1222-1237.

Araujo, A. C. B., Hori, E. S. \& Skogestad, S., 2007b. Application of Plant-Wide Control to the HDA Process. II - Regulatory Control. Indutrial \& Engineering Chemical Research, Volume 46, pp. 5159-5174. 
Arkun, Y. \& Downs, J., 1990. A general method to calculate input-output gains and the relative gain array for integrating processes. Computers \& Chemical Engineering, Volume 14, Issue 10, pp. 1101-1110.

Balchen, J. G. \& Mummé, K. I., 1988. Process Control, Structures and applications. Van Nostrand Reinhold.

Baldea, M., Araujo, A., Skogestad, S. \& Daoutidis, P., 2008. Dynamic Considerations in the Synyhesis of Self-Optimizing Control Structures. AIChE Journal, Volume 54, pp. 1830-1841.

Banerjee, A. \& Arkun, Y., 1995. Control Configuration Design Applied to the Tennessee Eastman Plant-Wide Control Problem. Computers \& Chemical Engineering, Volume 19, pp. 453-480.

Bathelt, A., Ricker, N. L. \& Jelali, M., 2015. Revision of the Tennessee Eastman Process Model. IFAC-PapersOnLine, Volume 48, Issue 8, pp. 309-314.

Baughman, D. R. \& Liu, Y. A., 1995. Neural networks in bioprocessing and chemical engineering. San Diego: Academic Press.

Bellman, R. E., 1957. Dynamic Programming. Princeton, NJ: Princeton University Press.

Bildea, C. S. \& Dimian, A. C., 2003. Fixing Flow Rates in Recycle Systems: Luyben's Rule Revisited. Industrial \& Engineering Chemistry Research, Volume 42, pp. 45784585.

Bouton, G. R. \& Luyben, W. L., 2008. Optimum Economic Design and Control of a Gas Permeation Membrane Coupled with the HDA Process. Industrial \& Engineering Chemical Research, Volume 647, pp. 1221-1237.

Bristol, E. H., 1966. On a new measure of interactions for multivariable process control. IEEE Transactions on Automatic Control, Volume AC-11, pp. 133-134.

Buckley, P. S., 1964. Thechniques of Process Control. New York: Wiley. 
Byeongsik, K., Tongue, B. H. \& Packard, A., 1994. A method for determining the optimal location of a distributed sensor/actuator. Shock and Vibration, Volume 1, Issue 4, pp. 357-374.

Cao, Y. \& Kariwala, V., 2008. Bidirectional Branch and Bound for Controlled Variable Selection. Part 1. Principles and Minimum Singular Value Criterion. Computers \& Chemical Engineering, Volume 32, Issue 10, pp. 2306-2319.

Cao, Y. \& Rossister, D., 1997. An Input Pre-Screening Technique for Control Structure Selection. Computers \& Chemical Engineering, Volume 21, pp. 563-569.

Cao, Y. \& Saha, P., 2005. Improved Branch and Bound Method for Control Structure Screening. Chemical Engineering Science, Volume 60, pp. 1555-1564.

Castro, J. J. \& Doyle, F. J., 2002. Plantwide Control of the Fiber Line in a Pulp Mill. Industrial and Chemistry Engineering Research, Volume 41, pp. 1310-1320.

Castro, J. J. \& Doyle, F. J., 2004a. A pulp mill benchmark problem for control: problem description. Journal of Process Control, Volume 14, pp. 17-29.

Castro, J. J. \& Doyle, F. J., 2004b. A pulp mill benchmark problem for control: Application of plantwide control design. Journal of Process Control, Volume 14, pp. 329-347.

Charnes, A. \& Cooper, W. W., 1961. Management models and industrial applications of linear programming. New York: Wiley.

Charnes, A., Cooper, W. W. \& Ferguson, R., 1955. Optimal estimation of executive compensation by linear programming. Management Science, Volume 1, pp. 138-151.

Cheng, R., McAvoy, T. \& Zafirou, E., 2004. Plantwide Control System Design: Extension to Multiple-Forcing and Multiple-Steady-State Operation. Industrial \& Engineering Chemical Research, Volume 43, pp. 3685-3694.

Chen, R. \& McAvoy, T. J., 2003. Plantwide control system design: Methodology and application to a vinyl acetate process. Industral \& Engineering Chemistry Research, Volume 20, Issue 42, pp. 4753-4771. 
Chen, R., McAvoy, T. \& Zafiriou, E., 2003. Plant-Wide Control System Design: Extension to Multiple-Forcing and Multiple-Steady-State Operation. Industrial \& Engineering Chemistry Research, Volume 42, pp. 4586-4598.

Dimian, A. C., Groenendijk, A. J. \& ledema, P. D., 2001. Recycle Interaction Effects on the Control of Impurities in a Complex Plant. Industrial \& Engineering Chemistry Research, Volume 40, pp. 5784-5794.

Douglas, J. M., 1988. Conceptual Design of Chemical Processes. New York: McGrawHill.

Downs, J. J., 1992. Distillation control in a plantwide control enviroment. In: W. L. Luyben, ed. Practical Distillation Control. New York: Van Nostrand Reinhold, pp. 413439.

Downs, J. J. \& Skogestad, S., 2011. An industrial and academic perspective on plantwide control. Annual Reviews in Control, Volume 35, pp. 99-110.

Downs, J. J. \& Vogel, E. F., 1993. A Plant-Wide Industrial Process Control Problem. Computers \& Chemical Engineering, Volume 17, pp. 245-255.

Engell, S., 2007. Feedback control for optimal process operation. Journal of Process Control, Volume 17, pp. 203-219.

Erickson, K. T. \& Hedrick, J. L., 1999. Plant-Wide Process Control. New York: WileyInterscience.

Findeisen, W. et al., 1980. Control and coordination in Hierarquical Systems. John Wiley \& Sons.

Fonyo, Z., 1994. Design Modifications and Proper Plant-Wide Control. Computers \& Chemical Engineering, Volume 18, pp. S483-S492.

Foss, A. S., 1973. Critique of chemichal process control theory. AlChE Journal, Volume 19, pp. 209-214.

Garcia, C., 2005. Modelagem e Simulação de Processos Industriais e de Sistemas Eletromecânicos. 2nd ed. São Paulo(SP): EDUSP. 
Garcia, C., Potts, A. S., Juliani, R. C. G., Alves, V. A. O.; da Silva, T. S., 2014. Algorithms and Methods for Identification of Multivariable Plants. In: Computer Aided Chemical Engineering. Elsevier, pp. 667-672.

Gernaey, K. V., Glassey, J., Skogestad, S., Krämer, S., Weiß, A., Engell, S., Pistikopoulos, E. N., Cameron, D. B., 2012. Process System Engineering, 5. Process Dynamics, Control, Monitoring and Identification. In: Ullmann's Encyclopedia of Industrial Chemistry. Weinheim: Wiley-VCH Verlag GmbH \& Co. KGaA, pp. 10-24.

Groenendijk, A. J., Dimian, A. C. \& ledema, P. D., 2000. Systems Approach for Evaluating Dynamics and Plant-Wide Control of Complex Plants. AIChE Journal, Volume 46, pp. 133-145.

Grosdidier, P., Mason, A., Aitolahti, A., Heinonen, P., Vanhamäki, V., 1993. FCC unit Reactor-Regenerator Control. Computers \& Chemical Engineering, Volume 17, Issue 2, pp. 165-179.

Halvorsen, I. J., Skogestad, S., Morud, J. C. \& Alstad, V., 2003. Optimal selection of coontrolled variables. Industrial \& Engineering Chemical Research, Volume 42, Issue 14, pp. 3273-3284.

Hansen, J. E., Jorgensen, S. B., Heath, J. \& Prekins, J., 1998. Control structure selection for energy integrated distilattion column. Journal of Process Control, Volume 8, pp. 185-195.

Haykin, S., 2009. Neural Networks and Learning Machines. 3rd ed. Pearson.

Heath, J. A., Kookos, I. K. \& Perkins, J. D., 2000. Process control structure based on economics. AIChE Journal, Volume 46, Issue 10, pp. 1998-2016.

Herrmann, G., Spurgeon, S. K. \& Edwards, C. A., 2003. Model-based Sliding Mode Control Methodology Applied to the HDA Plant. Journal of Process Control, Volume 13, pp. 129-138.

Hespanha, J. P., 2009. Linear Systems Theory. Princeton: Princeton.

Hoo, K. A., 2010. Plantwide Control. In: The Control Handbook - Control System Applications. 2nd ed. Boca Raton(FL): CRC Press, pp. 17-8:17-18. 
Ignizio, J. P., 1976. Goal programming and extensions. Lexington, MA: Lexington Books.

ljiri, Y., 1965. Management Goals and Accounting for Control. North-Holland.

Jäschke, J., Cao, Y., Kariwala, V., 2017. Self-optimizing control - A Survey. Annual Reviews in Control, Volume 43, pp.199-223.

Jockenhövel, T., Biegler, L. T. \& Wächter, A., 2003. Dynamic optimization of the Tennessee Eastman process using the OptControlCentre. Computers \& Chemical Engineering, Volume 11, Issue 27, pp. 1513-1531.

Jones, D. \& Tamiz, M., 2010. Practical Goal Programming. Springer.

Jørgensen, J. B. \& Jørgensen, S. B., 2000. Towards Automatic Decentralized Control Structure Selection. Computers \& Chemical Engineering, Volume 24, Issues 2-7, pp. 841-846.

Juliani, R. C. G., 2012. Sintonia Ótima de Controladores, São Paulo, Masters Dissertation. Polytechnic School of the University of São Paulo, São Paulo.

Juliani, R. C. G., Alves, V. A. O. \& Garcia, C., 2013. Time Delay: an alternative definition for optimal System Identification. In: Proceedings of the 52nd IEEE Conference on Decision and Control, CDC 2013, Firenze, Italy, 2013. pp. 3894-3899.

Juliani, R. C. G. \& Garcia, C., 2012. Optimal Multi-Objective and Contrained PID Tuning. In: Anais do XIX Congresso Brasileiro de Automática, CBA 2012, Campina Grande, PB, Brazil, 2012. pp. 5456-5463.

Juliani, R. C. G. \& Garcia, C., 2014. Optimal Multi-Objective Multivariable PID Tuning. In: Anais do XX Congresso Brasileiro de Automática, CBA 2014, Belo Horizonte, MG, Brazil, 2014. pp. 1096-1103.

Juliani, R. C. G. \& Garcia, C., 2017a. Applications of an Optimal Multi-Objective Technique for Integrated Control Structure Selection and Tuning. In: Proceedings of 20th World Congress of the International Federation of Automatic Control, IFAC 2017, Toulouse, France, 2017. 
Juliani, R. C. G. \& Garcia, C., 2017b. Plantwide Control: A Review of Design Techniques, Benchmarks and Challenges. Industrial \& Engineering Chemistry Research, Volume 56, Issue 28, pp. 7877-7887.

Kariwala, V. \& Cao, Y., 2009. Bidirectional branch and bound for controlled variable selection. Part II: Exact local method for self-optimizing control. Computers \& Chemical Engineering, Volume 33, pp. 1402-1412.

Kariwala, V. \& Yi, C., 2010. Branch and bound methods for multiobjective pairing selection. Automatica, Volume 46, pp. 932-936.

Kirk, D. E., 1970. Optimal Control Theory: An Introduction.Prentice Hall.

Konda, N. V. S. N. M., Ragaiah, G. P. \& Krishnaswamy, P. R., 2005. Plantwide control of industrial processes: An integrated framework of simulation and heuristics. Industrial \& Engineering Chemistry Research, Volume 42, pp. 8300-8313.

Konda, N. V. S. N. M., Rangaiah, G. P. \& Lim, D. K. H., 2006. Optimal Process Design and Effective Plant-Wide Control of Industrial Processes by a Simulation-Based Approach. Industrial \& Engineering Chemistry Research, Volume 45, pp. 5955-5970.

Kookos, I. K. \& Perkins, J. D., 2001. Heuristic-Based Mathematical Programming Framework for Control Structure Selection. Industrial \& Engineering Chemistry Research, Volume 40, pp. 2079-2088.

Kookos, I. K. \& Perkins, J. D., 2002. An Algorithm method for selection of multivariable process control structures. Journal of Process Control, Volume 12, pp. 85-99.

Larsson, L., Hestetun, K., Hovland, E. \& Skogestad, S., 2001. Self-optimizing control of a large-scale plant: the Tennessee Eastman process. Industrial \& Engineering Chemistry Research, Volume 40, pp. 4889-4901.

Larsson, T. \& Skogestad, S., 1998. Limitations imposed by lower layer control configurations. Proceedings of the AIChE Annual Meeting, 16-20 November.

Larsson, T. \& Skogestad, S., 2000. Plantwide control: A review and a new design procedure. Modeling, Identification and Control, Volume 21, pp. 209-240.

Lee, S. M., 1972. Goal programming for decision analysis. Auerback, Philadelphia. 
Ljung, L., 1999. System Identification: Theory for the User. 2nd ed. Upper Saddle River, NJ: Prentice Hall.

Luppi, P. A., Zumoffen, D. A. R. \& Basualdo, M. S., 2013. Decentralized plantwide control strategy for large-scale processes. Case study: pulp mill benchmark problem. Computers \& Chemical Engineering, Volume 52, pp. 272-285.

Luppi, P., García, M., Zumoffen, D. \& Basualdo, M., 2011. Computer aided tools for designing plant-wide control structures for large-scale industrial processes. Iberoamerican Journal of Industrial Engineering, Volume 3, Issue 2, pp. 20-35.

Luyben, M. L. \& Luyben, W. L., 1995. Design and Conytol of a Complex Process Involving Two Reaction Steps, Three Distillation Columns, and Two Recycle Streams. Industrial \& Engineering Chemistry Research, Volume 34, pp. 3885-3898.

Luyben, M. L., Tyreus, B. D. \& Luyben, W. L., 1997. Plantwide Control Design Procedure. American Institute of Chemical Engineering Journal, Volume 43, pp. 31613174.

Luyben, W. L., 1989. Process Modeling, Simulation and Control for Chemical Engineers. 2nd ed. USA: Mcgraw Hill.

Luyben, W. L., 1993. Dynamics and Control of Recycle Systems. 1. Simple Open-Loop and Closed-Loop Systems. Industrial \& Engineering Chemistry Research, Volume 32, pp. 446-475.

Luyben, W. L., 1994. Snowball effects in reactor/separator processes with recycle. Industrial \& Engineering Chemistry Research, February, Volume 33, Issue 2, pp. 299305.

Luyben, W. L., 2002. Plant-Wide Dynamic Simulators in Chemical Processing and Control. New York: Marcel Dekker.

Luyben, W. L., Tyreus, B. D. \& Luyben, M. L., 1998. Plantwide Process Control. New York: McGraw-Hill.

Lyman, P. R. \& Georgakis, C., 1995. Plant-Wide Control of the Tennessee Eastman Problem. Computers \& Chemical Engineering, Volume 19, pp. 321-331. 
Marcangöz, M. \& Doyle, F. J., 2008. Real-time optimization of the pulp mill benchmark problem. Computers \& Chemical Engineering, Volume 32, pp. 789-804.

Marler, R. T. \& Arora, J. S., 2004. Survey of multi-objective optimization methods for engineering. Structural and Multidisciplinary Optimization, 23 March, Volume 26, Issue 6, pp. 369-395.

McAvoy, T. J., 1999. Synthesis of Plant-Wide Control Systems Using Optimization. Industrial \& Engineering Chemistry Research, Volume 38, pp. 2984-2994.

McAvoy, T. J. \& Ye, N., 1994. Base Control for the Tennessee Eastman Problem. Computers \& Chemical Engineering, Volume 18, pp. 383-413.

McAvoy, T. J., Ye, N. \& Gang, C., 1996. Nonlinear Inferential Parallel Cascade Control. Industrial \& Engineering Chemistry Research, Volume 35, pp. 130-137.

Mercangöz, M. \& Doyle, F. J., 2006. Model-Based Control in the Pulp and Paper Industry: Caharacterizing the Process from the Digester to the Paper Machine. IEEE Control Systems Magazine, August. pp. 30-39.

Molina, G. D., Zumoffen, D. A. R. \& Basualdo, M. S., 2011. Plant-wide control strategy applied to the Tennessee Eastman process operating points. Computers \& Chemical Engineering, Volume 35, Issue 10, pp. 2081-2097.

Monteiro, L. H. A., 2006. Sistemas Dinâmicos. São Paulo(SP): Livraria da Física.

Morari, M., 1982. Integrated plant control: A solution at hand or a research topic for the. CPC-II, pp. 467-495.

Morari, M., Stephanopoulos, G. \& Arkun, Y., 1980. Studies in the synthesis of control structures for chemical processes. Part I. Formulation of the problem. Process decomposition and the classification of the control task. Analysis of the optimizing control structures. AIChE Journal, Volume 26, pp. 220-232.

Müller, P. C. \& Weber, H. I., 1972. Analysis and optimization of certain qualities of controllability and observability for linear dynamical systems. Automatica, Volume 8, pp. 237-246. 
Narraway, L. \& Perkins, J., 1994. Selection of process control structure based on economics. Computers \& Chemical Engineering, Volume 18, Issue SUPP 1, pp. S511S515.

Narraway, L. T. \& Perkins, J. D., 1993. Selection of control strucuture based on linear dynamic economics. Computers \& Chemical Engineering, Volume 32, Issue 11, pp. 2681-2692.

Ng, C. S. \& Stephanopoulos, G., 1996. Synthesis of Control Systems for Chemical Plants. Computers \& Chemical Engineering, Volume 20, pp. S999-S1004.

Niderlinski, A., 1971. A heuristic approach to the design of linear multivariable interacting control systems. Automatica, Volume 7, pp. 691-701.

Norris, G. A. \& Skelton, R. E., 1989. Selection of dynamic sensors and actuators in the control of linear systems. Journal of Dynamic Systems, Measurements, and Control, September, Volume 111, pp. 389-397.

Ochoa, S., Wozny, G. \& Repke, J.U., 2010. Plantwide Optimizing Control of a continuous bioethanol production process. Journal of Process Control, Volume 20, pp. 983-998.

Ogata, K., 2009. Modern Control Engineering. 5th ed. Prentice Hall.

Ogunnaike, B., Lemaire, J. P., Morari, M. \& Ray, W. H., 1983. Advanced multivariable control of a pilot-plant distillation column. AIChE Journal, Volume 29, Issue 4, pp. 632640.

Olsen, D. G., Svrcek, W. Y. \& Young, B. R., 2005. Plant-Wide Control Study of a Vinyl Acetate Monomer Process Design. Chemical Engineering Communications, Volume 192, pp. 1243-1257.

Ponton, J. W. \& Laing, D. M., 1993. A Hierarchical Approach to Design of Process Control Systems. Transactions of the institute of Chemical Engineering, Volume 71, pp. 181-188.

Prett, D. M. \& Morari, M., 1987. Shell Process Control Workshop. Butterworth, Stoneham, MA. 
Price, R. M., Lyman, P. R. \& Georgakis, C., 1994. Throughput Manipulation in PlantWide Control Structures. Industrial \& Engineering Chemistry Research, Volume 33, pp. 1197-1207.

Psaltis, A., Kookos, I. K. \& Kravaris, C., 2013. Plant-wide control structure selection methodology based on economics. Computers \& Chemical Engineering, Volume 52, pp. 240-248.

Psaltis, A., Kookos, I. K. \& Kravaris, C., 2014. Plantwide control structure design methodology for the benchmark vinyl acetate monomer plant. Computers \& Chemical Engineering, Volume 62, pp. 108-116.

Qiu, Q. F., Rangaiah, G. P. \& Krishnaswamy, P. R., 2003. Application of a Plant-Wide Control Design to the HDA Process. Computers \& Chemical Engineering, Volume 27, pp. 73-94.

Rangaiah, G. P. \& Kariwala, V. eds., 2012. Plantwide Control: Recent Developments and Applications. Chichester: Wiley.

Rawlings, J. R. \& Steward, B. T., 2008. Coordination multiple optimization-based controllers: new opportunities and challenges. Journal of Process Control, Volume 18, pp. 839-845.

Reeves, D. E., 1991. A comprehensive approach to control configuration design for complex systems. Ph.D. thesys presented to the Georgia Tech University.

Ricker, N. L., 1996. Decentralized Control of The Tennessee Eastman Challenge Problem. Journal of Process Control, Volume 6, pp. 205-221.

Ricker, N. L. \& Lee, J. H., 1995. Non-Linear Model-Predictive Control of the Tennessee Eastman Challenge Process. Computers \& Chemical Engineering, Volume 19, pp. 961-981.

Rijnsdorp, J. E., 1991. Integrated Process Control and Automation. Elsevier.

Rinard, I. H. \& Downs, J. J., 1992. Plant wide control: A review and critique. New Orleans, , p. $67 f$. 
Robinson, D., Chen, R., McAvoy, T. \& Schnelle, P. D., 2001. An Optimal Control Based Approach to Designing Plant-Wide Control System Architectures. Journal of Process Control, Volume 11, pp. 223-236.

Samar, R. \& Postlethwaite, I., 1994. Multivariable controller design for a high performance aero-engine. Coventry, UK, pp. 1312-1317.

Seider, W. D., Seader, J. D. \& Lewin, D. R., 2004. Part Four: Plantwide Controllability Assessment. In: Product \& Process Design Principles. 2nd ed. Wiley.

Sharifzadeh, M. \& Thornhill, N. F., 2012. Optimal selection of control structure using a steady-state inversely controlled process model. Computers \& Chemical Engineering, 5 March, Volume 38, pp. 126-138.

Shinskey, F. G., 1984. Distillation Control: For Productivity and Energy Conservation. 2nd Edition ed. New York: McGraw-Hill.

Skogestad, S., 2000a. Plantwide control: The search for the self-optimizing control structure. Journal of Process Control, Volume 10, pp. 487-507.

Skogestad, S., 2000b. The Missing Link between Steady-State Optimization and Control. Computers \& Chemical Engineering, Volume 24, pp. 569-575.

Skogestad, S., 2002. Plantwide control: Towards a systematic procedure. The Hague, The Netherlands, Elsevier.

Skogestad, S., 2004a. Control structure for complete chemical plants. Computers \& Chemical Engineering, Volume 28, Issues 1-2, pp. 219-234.

Skogestad, S., 2004b. Near-optimal operation by self-optimizing control: from process control to marathon running and business systems. Computers \& Chemical Engineering, Volume 29, pp. 127-137.

Skogestad, S., 2012. Economic Plantwide Control. In: Plantwide Control - Recent Developments and Applications. Wiley, pp. 229 - 251.

Skogestad, S., Jacobsen, E. W. \& Morari, M., 1990. Inadequacy of Steady-State Analysis for Feedback Control: Distillate-Bottom of Distillation Columns. Industrial \& Engineering Chemistry Research, Volume 29, pp. 2339-2346. 
Skogestad, S., Manfred, M. \& Doyle, J. C., 1988. Robust Control of III-Conditione Plants: High-Purity Distillation. IEEE Transactions on Automatic Control, Volume 33, Issue 12, pp. 1092-1105.

Skogestad, S. \& Postlethwaite, I., 1996. Multivariable feedback control. New York: Wiley.

Stephanopoulos, G., 1983. Synthesis of control systems for chemical plants - a challenge for creativity. Computers \& Chemical Engineering, Volume 7, Issue 4, pp. 331-365.

Stephanopoulos, G., 1984. Chemical Process Control. New Jersey: Prentice Hall.

Stephanopoulos, G. \& Ng, C., 2000. Perspectives on the synthesis of plant-wide control structures. Journal of Process Control, Volume 10, pp. 97-111.

Tian, Z. H. \& Hoo, K. A., 2005. Multiple Model-Based Control of the Tennessee Eastman Process. Industrial \& Engineering Chemistry Research, Volume 44, pp. 31873202.

Turkay, M., Gurkan, T. \& Ozgen, C., 1993. Synthesis of Regulatory Control Structures for a Styrene Plant. Computers \& Chemical Engineering, Volume 17, pp. 601-608.

Tyreus, B. D., 1998. Dominant variables for partial control. 2. Application to the Tennessee Eastman Challenge Process. Industrial \& Engineering Chemistry Research, Volume 38, pp. 1444-1455.

van de Wal, M. \& Jager, B., 1995. Control structure design: A survey. pp. 225-229.

van de Wal, M. M. J., 1994. Control structure design for dynamic systems: A review.

Vasbinder, E. M. \& Hoo, K. A., 2003. Decision-based approach to plantwide control structure synthesis. Industrial \& Engineering Chemistry Research, Volume 42, pp. 4586-4598.

Vasbinder, E. M., Hoo, K. A. \& Mann, U., 2004. Synthesis of a Plant-Wide Control Structures using a Decision-Based Methodology. In: P. Seferlis \& M. C. Georgiadis, eds. The Integration of Process Design and Control. New York: Elsevier, pp. 375-400. 
Vasudevan, S., Rangaiah, G. P., Konda, N. V. S. N. M. \& Tay, W. H., 2009. Application and Evaluation of Three Methodologies for Plantwide Control of the Styrene Monomer Plant. Industrial \& Engineering Chemistry Research, Volume 48, pp. 10941-10961.

Wang, P. \& McAvoy, T., 2001. Synthesis of Plant-Wide Control Systems Using a Dynamic Model and Optimization. Industrial \& Engineering Chemistry Research, Volume 40, pp. 5732-5742.

Ward, J. D., Mellichamp, D. A. \& Doherty, M. F., 2006. Insight from economically optimal steady-state operating policies for dynamic plantwide control. Industrial \& Engineering Chemistry Research, Volume 45, p. 1343.

Wood, R. K. \& Berry, M. W., 1973. Terminal composition control of a binary distillation column. Chemical Engineering Science, Volume 28, pp. 1707-1717.

Ye, N., McAvoy, T. J., Kosanovich, K. A. \& Piovoso, M. J., 1995. Optimal Averaging Level Control for the Tennessee Eastman Problem. Canadian Journal of Chemical Engineering, Volume 73, pp. 234-240.

Yi, C. \& Vinay, K., 2008. Bidirection branch and bound for controlled variable selection. Part I. Principles and minimun singular value criterion. Computers \& Chemical Engineering, Volume 32, pp. 2306-2319.

Zanin, A. C. \& Odloak, D., 1993. Advanced Control of an Atmospheric Resid Heater. In: Proceedings of the ISA First International Instrumentation, Systemas and Automation Meeting, Salvador, Bahia, 1993.

Zheng, A., Mahajanam, R. V. \& Douglas, J. M., 1999. Hierarchical procedure for plantwide control system synthesis. American Institute of Chemical Engineering Journal, Volume 45, Issue 6, pp. 1255-1265.

Zheng, H. Y., Wei, L. \& Jay, H. L., 1994. State Estimation based Model predictive Control Applied to Shell Control Problem: A Case Study. Chemical Engineering Science, Volume 49, Issue 3, pp. 285-301.

Zhu, G. Y. \& Henson, M. A., 2002. Model Predictive Control of Interconnected Linear and Non-Linear Processes. Industrial \& Engineering Chemical Research, Volume 41, pp. 801-816. 
Zhu, G. Y., Henson, M. A. \& Ogunnaike, B. A., 2000. A Hybrid Model Predictive Control Strategy for Non-Linear Plant-Wide Control. Journal of Process Control, Volume 10, pp. 449-458. 


\section{Appendix A - System Identification Procedure}

This appendix illustrates the procedure adopted for all the system identifications performed for this thesis. The identification of XMEAS 7 (reactor pressure) with the dataset obtained in the second identification experiment performed with the Tennessee Eastman Challenge process is shown.

The performed experiment starts with a period of 30 minutes with no changes in the setpoints to capture the plant conditions in nominal operation point, then, a set of step sequences is applied over a period of 12 hours to be used as validation data and, at last, GBN signals are applied to all setpoints for 24 hours, to capture datasets representative of the process dynamics.

\section{A.1 Identification Experiment}

For this experiment, the following PID setpoints were excited: SP 3, SP 4, SP 8 , SP 15 , SP 18 and SP 21 . The output identified in this detailed procedure is the XMEAS 7. The dataset collected from the experiment is shown in Figure A.1.
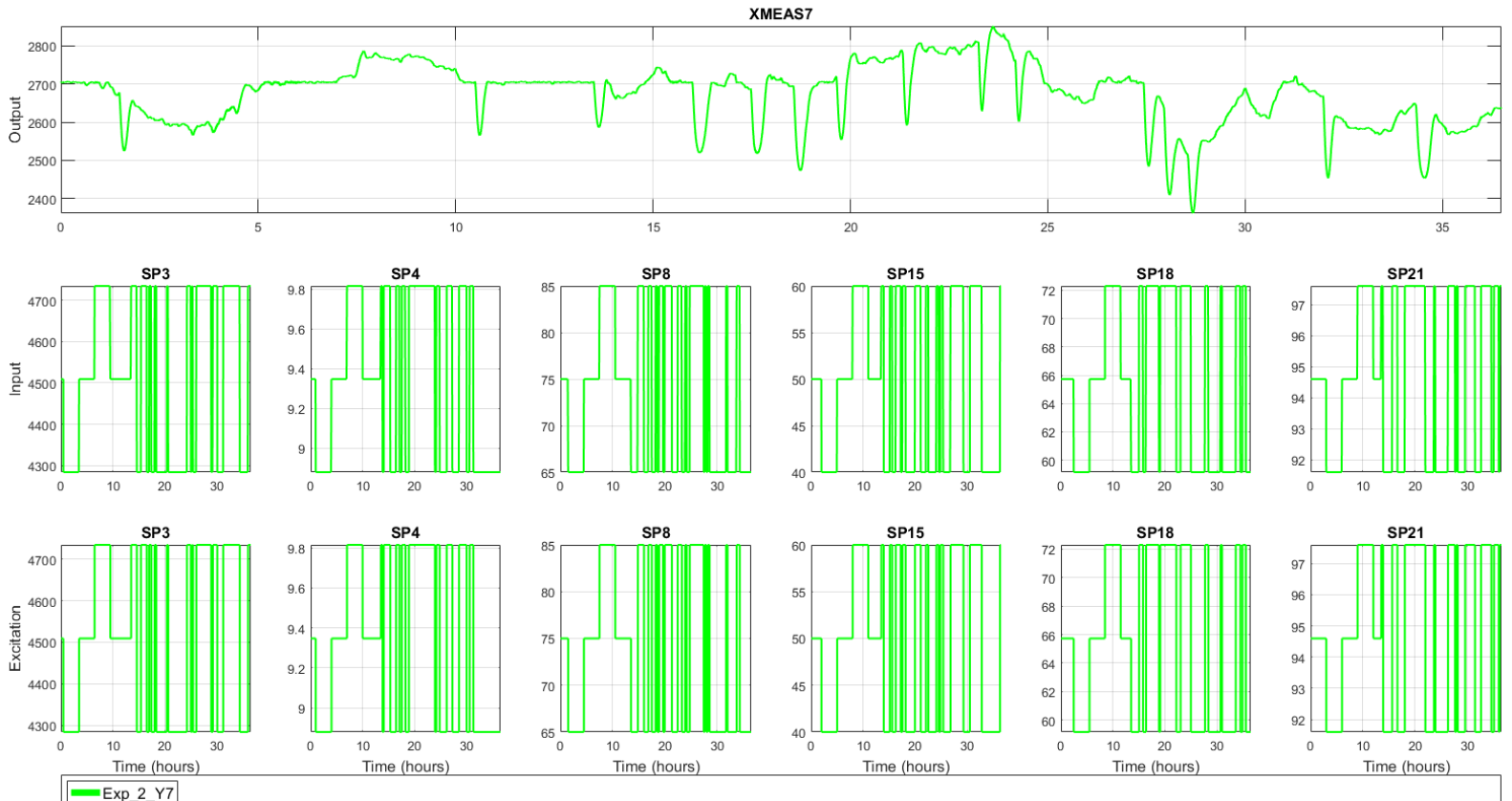

Figure A.1 - Original dataset. 


\section{A.2 Data Processing}

Before proceeding with the identification, the data needs to be pre-processed, to enhance the identification algorithms results.

First, a Phase 0 Butterworth filter is applied to the output signal to remove noise. Next, all signals are normalized to keep the origin of the incremental movements in 0 and equalize all amplitudes to 0.5 . The dataset obtained after these two operations is shown in Figure A.2.

Next, the best sampling time to be used in the identification is determined based on a correlation test and the dataset is resampled from 1 second to 60 seconds (1 minute). The resulting dataset is shown in Figure A.3, in which it can be noted that this operation causes no loss of information.
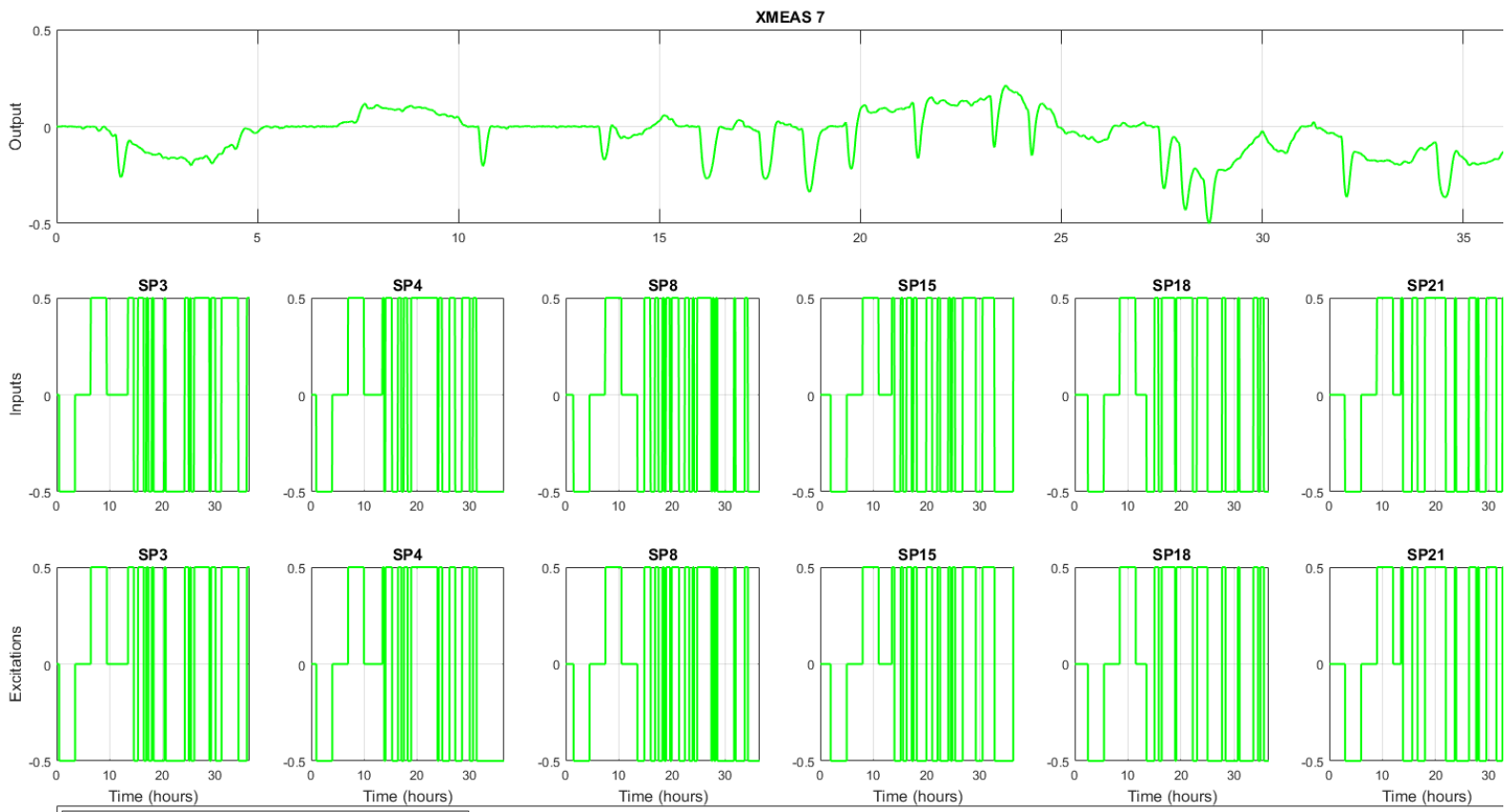

\begin{tabular}{cc}
10 & Time (hours) \\
\hline & Exp 2 Y7 FilteredButterworth_Phase_0 normalize
\end{tabular}

Figure A.2 - Filtered and normalized dataset. 

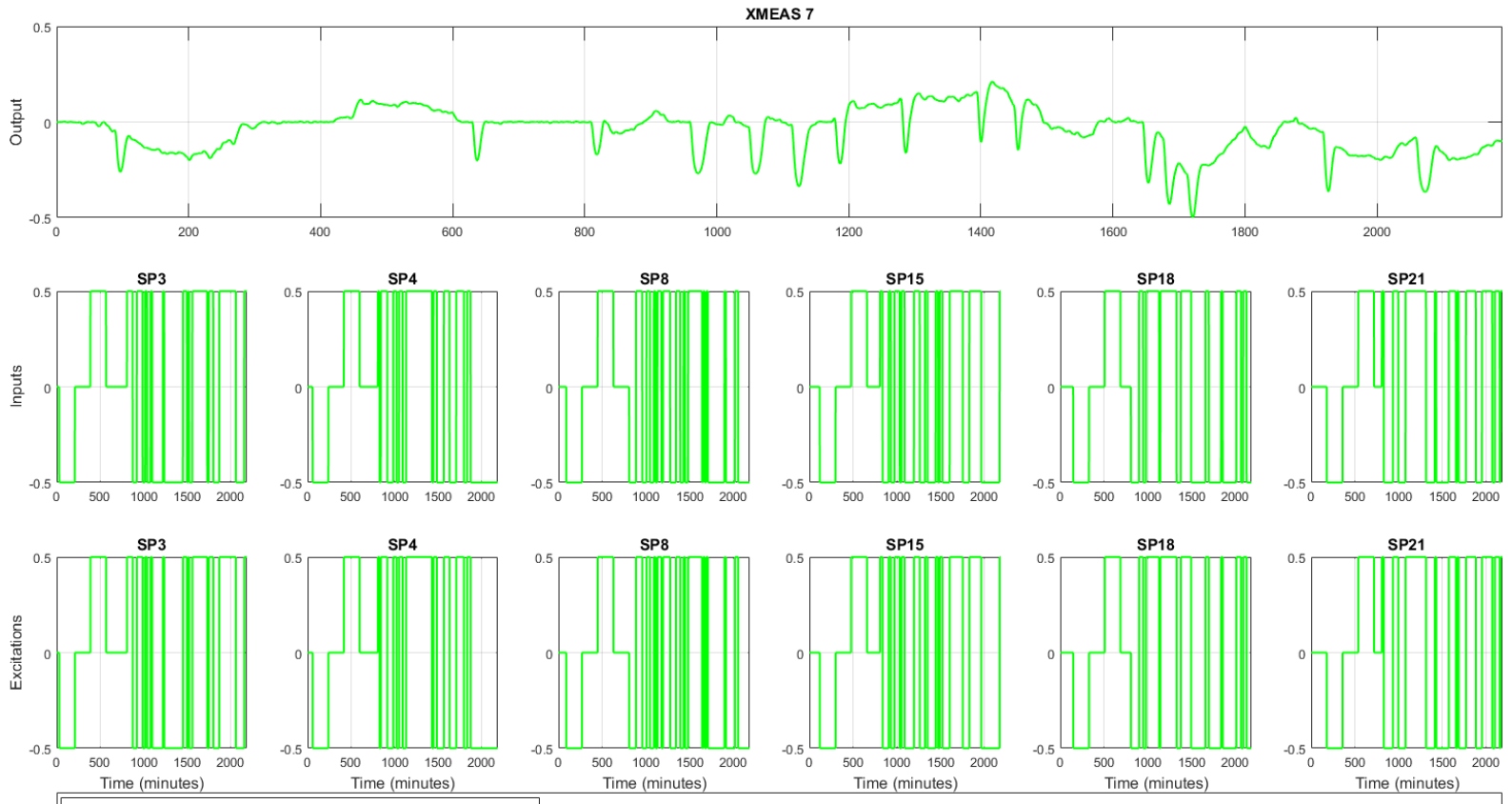

Figure A.3 - Resampled dataset.

\section{A.3 Datasets}

After the data is pre-processed and prepared to be used, it is separated into two datasets, one to be used in identification and another to be used in model validation. Figures A.4 and A.5 show the identification and validation datasets, respectively.
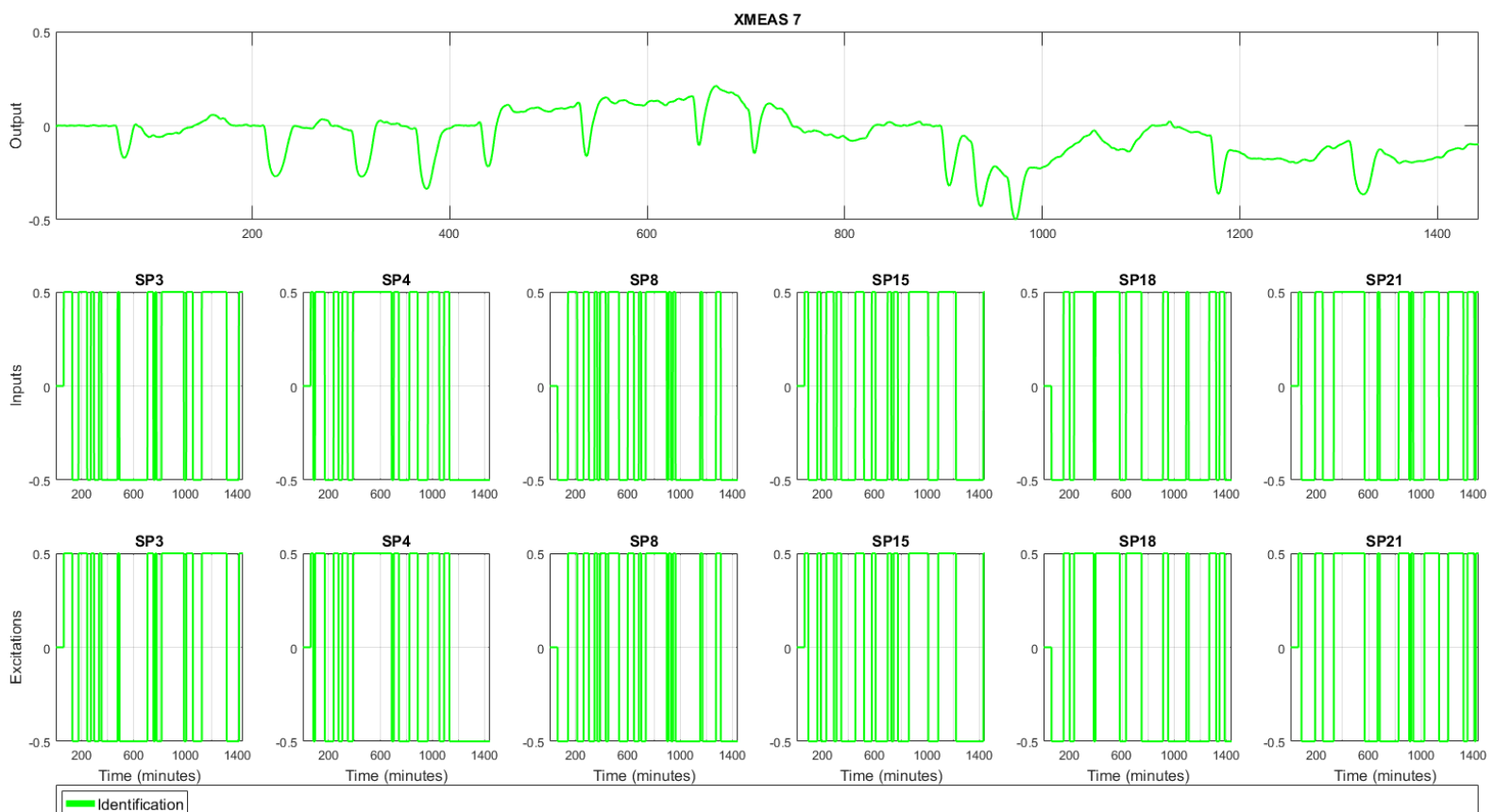

Figure A.4 - Identification dataset. 

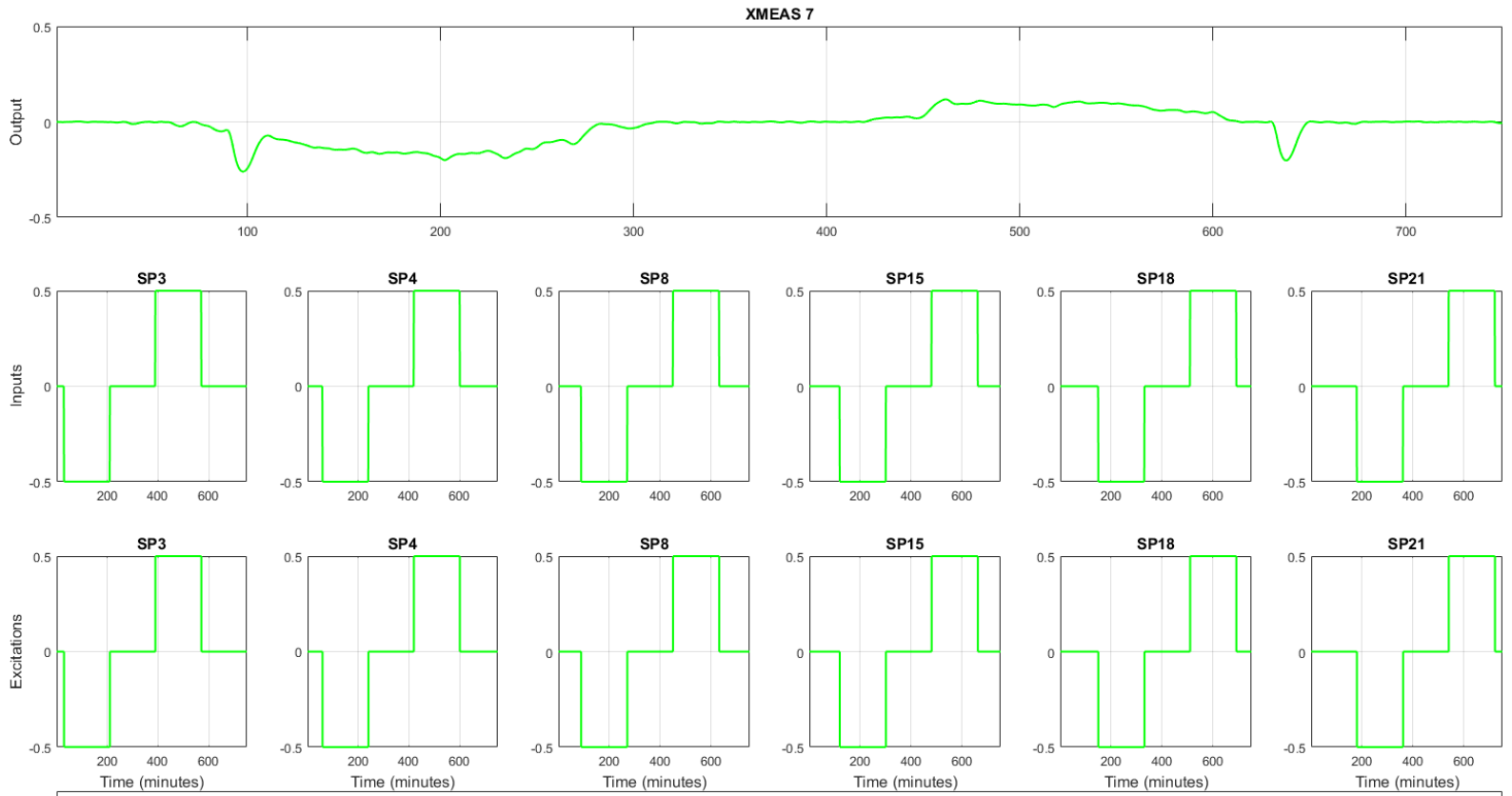

Figure A.5 - Validation dataset.

\section{A.4 Model Identification}

The identification starts with the determination of the no-model I/O relations, i.e., the determination of which process inputs affect the modeled output. For this variable, the resulting no-model I/O relation is $\left[\begin{array}{llllll}1 & 1 & 1 & 1 & 0 & 0\end{array}\right]$, which means that XMEAS 7 is affected by SP 3, SP 4, SP 8 and SP 15, but not by SP 18 or SP 21. This information is used in all subsequent identification steps.

Next, the OSOD algorithm is executed for all 29 available model structures to determine the best order and delay to be used in each structure.

After this step, the 29 candidate models are available and the best one need to be chosen.

\section{A.5 Model Validation and Selection}

Next, all identified models are evaluated using validation techniques. Each technique allows the rejection of some unsatisfactory models and provides a measurement to aid in the selection of the best model. 
Figure A.6 shows the distribution of the fit index for the non-rejected models. Such index indicates the fitting of the outputs predicted by the evaluated model to the validation dataset for different prediction steps.

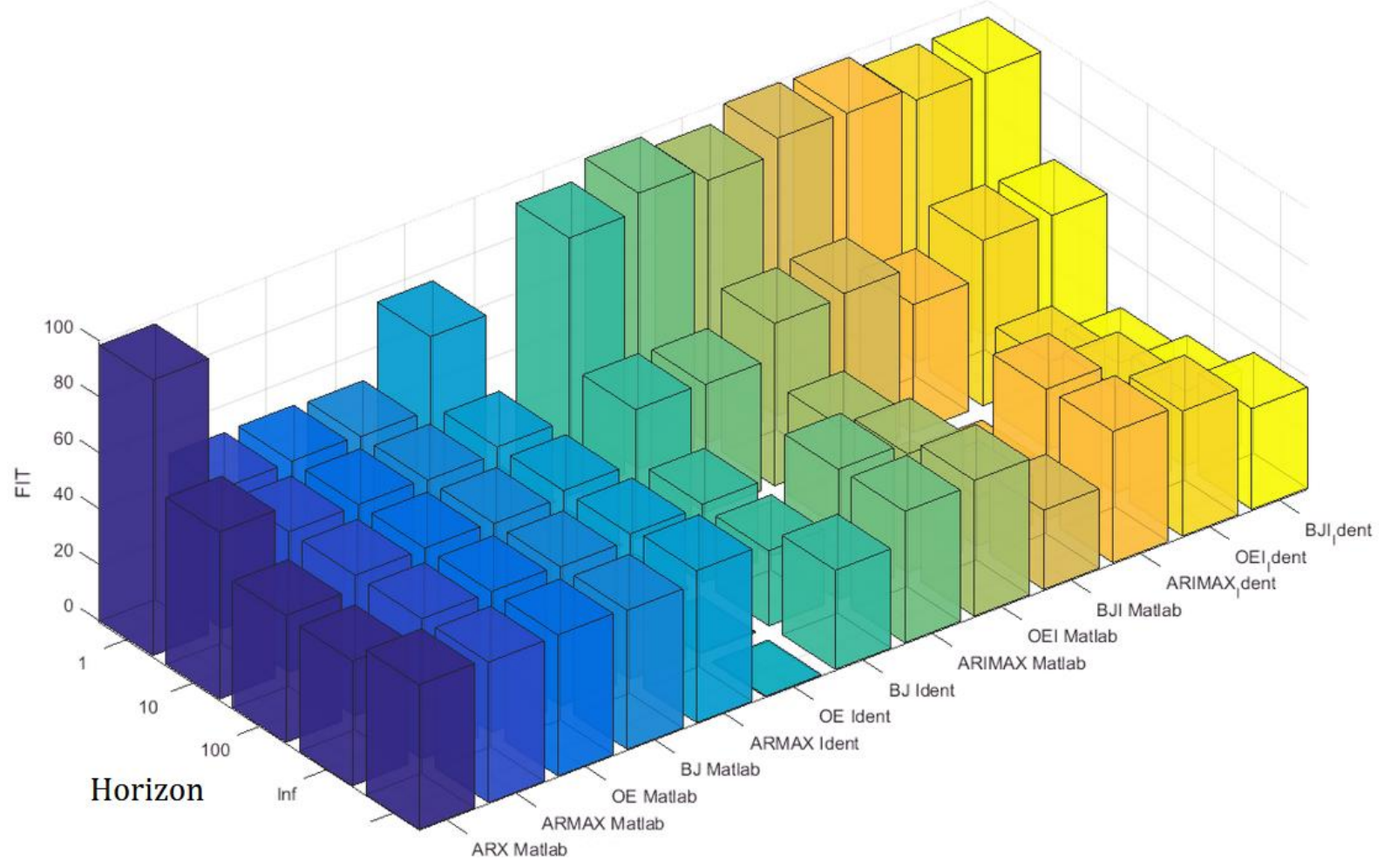

Figure A.6 - Fit index for the accepted models.

Figure A.7 shows the Theil indexes for the accepted models. This validation metric is similar to the fit index and it also indicates how well a predicted dataset is adjusted to the validation dataset.

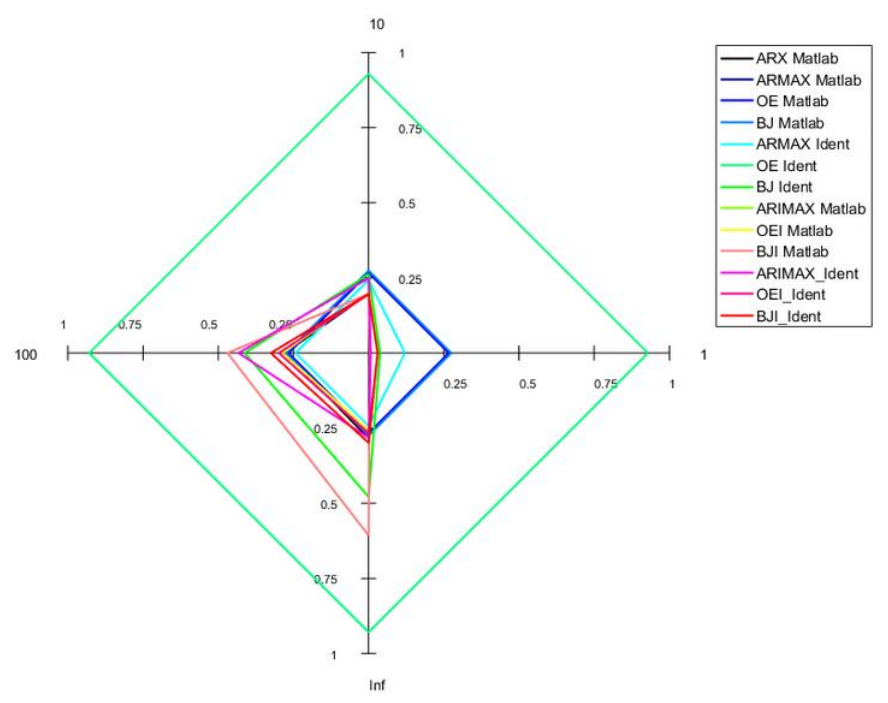

Figure A.7 - Theil Validation. 
The model gains are also observed to detect models that are too discrepant. Figure A.8 shows the gains for the accepted models.
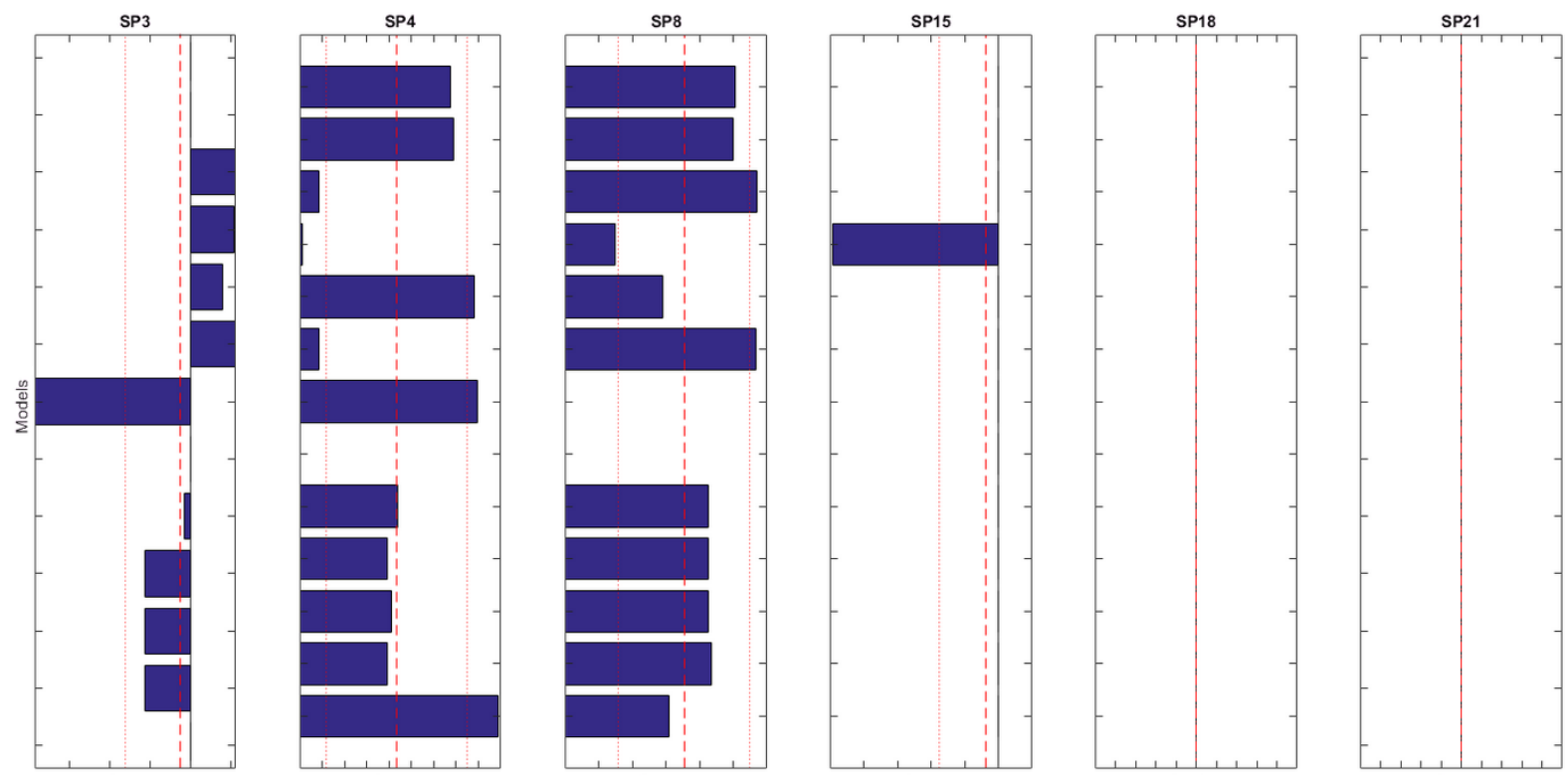

Figure A.8 - Model gain comparison.

Finally, the poles and zeros of the models are observed, as shown in Figure A.9.

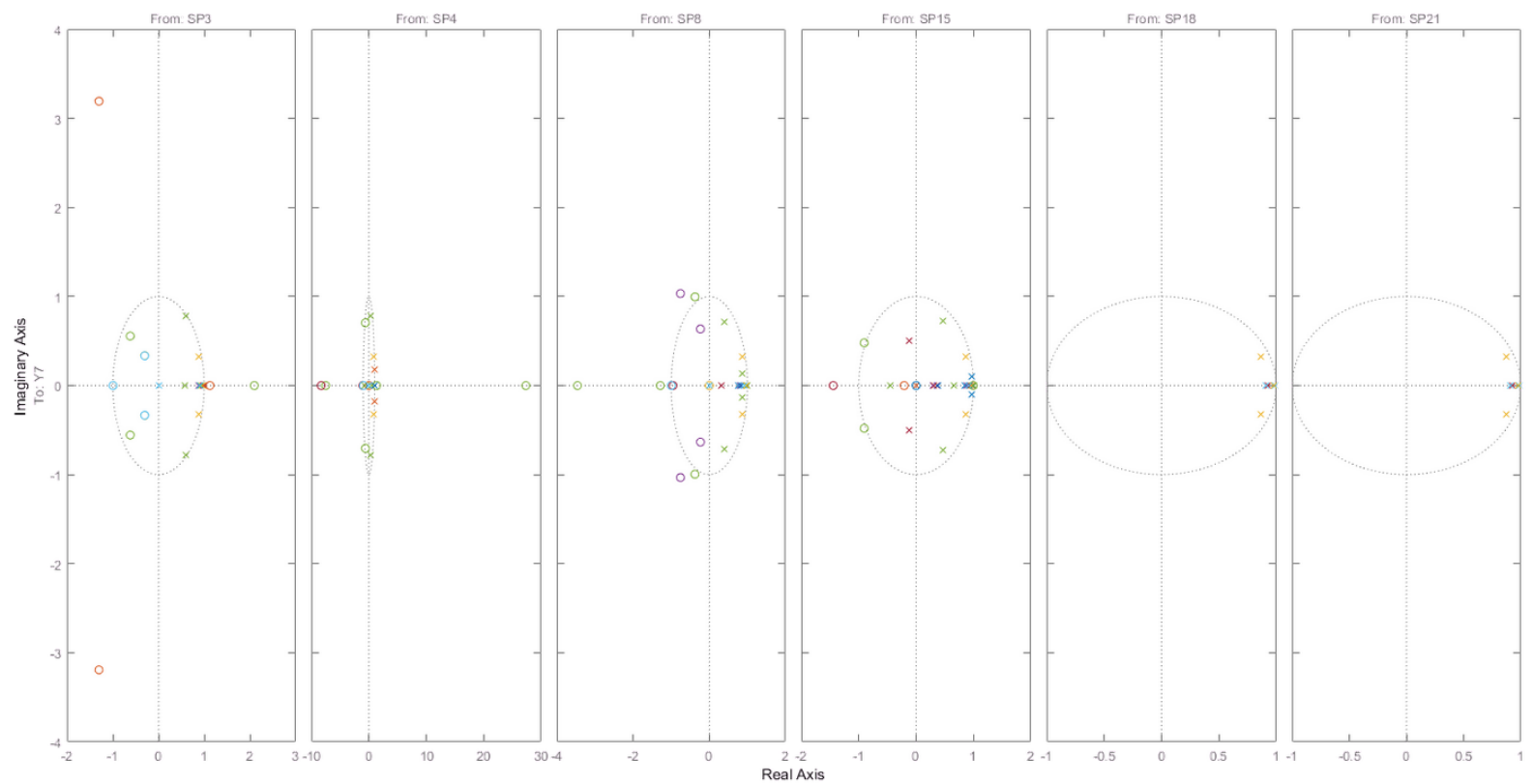

Figure A.9 - Model poles and zeros.

Based on the validation results, the models are classified and the BJ model structure is chosen as the most adequate for this process output. 


\section{A.6 Identified Model}

The BJ model structure was chosen for the output XMEAS 7 and the best model

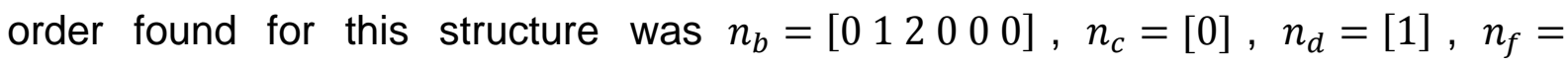
$\left[\begin{array}{llllll}0 & 1 & 3 & 0 & 0 & 0\end{array}\right]$ and $n_{k}=\left[\begin{array}{llllll}0 & 1 & 1 & 0 & 0 & 0\end{array}\right]$.

The resulting model performance is shown in Figure A.10 for the validation dataset and in Figure A.11 for the identification dataset.

The resulting model is archived and later denormalized and integrated with all the other identified models to build a single MIMO model for the whole plant. The final results are shown in Appendix B.
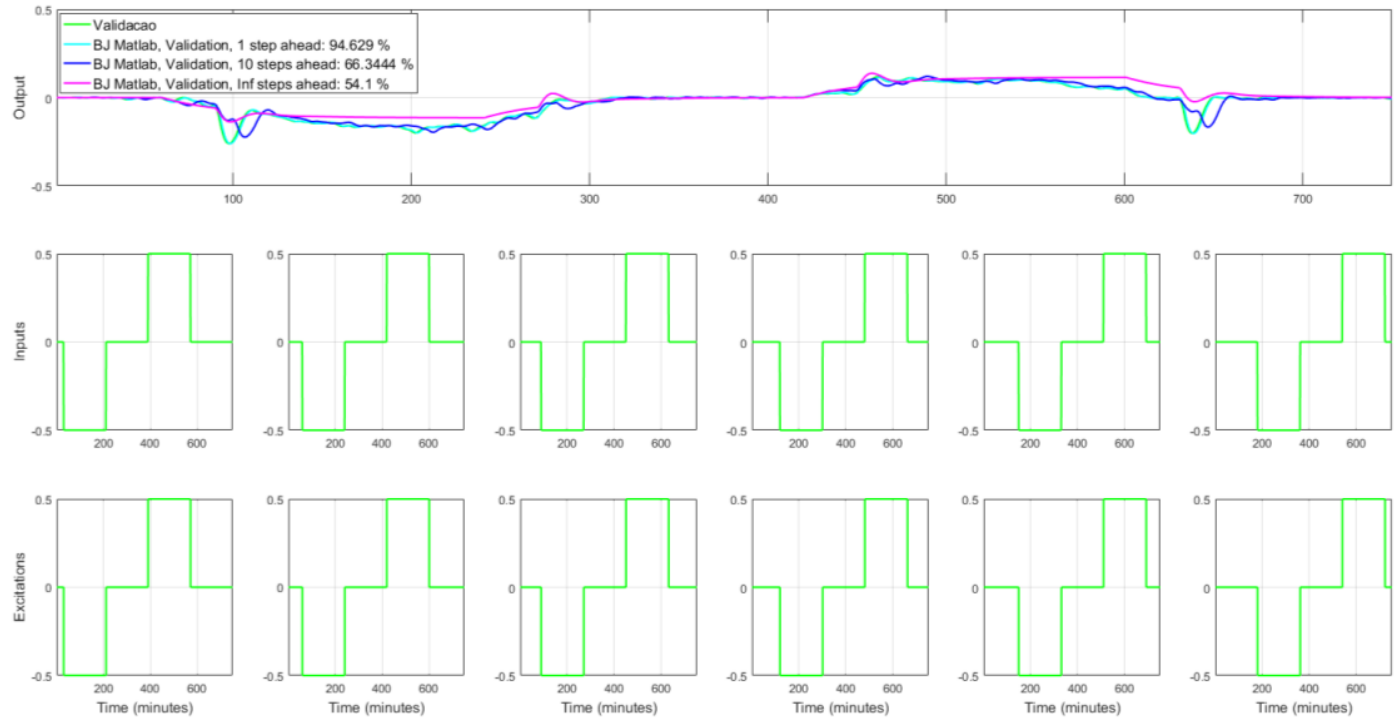

Figure A.10 - Final model performance for the validation dataset.
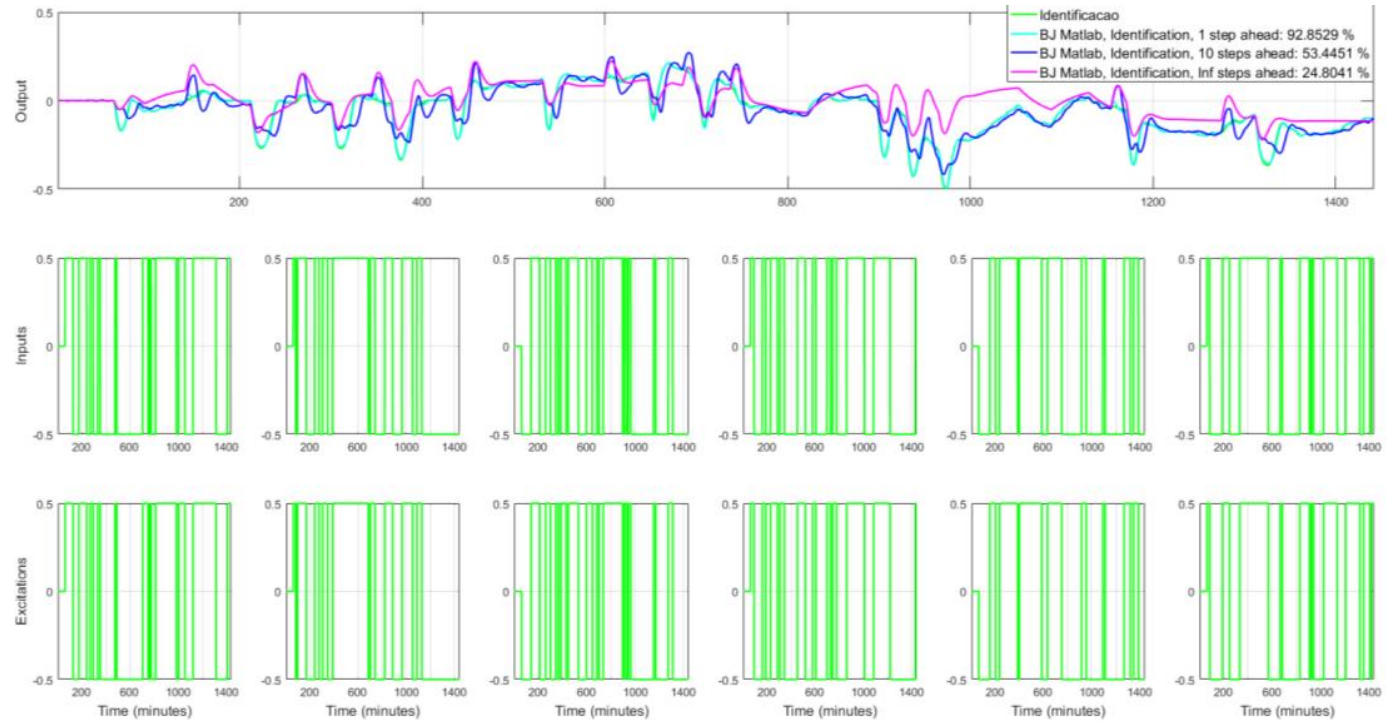

Figure A.11 - Final model performance for the identification dataset. 


\section{Appendix B - Identified Model for the Tennessee Eastman Challenge Process with Regulatory Control in Closed Loop}

The complete model for the process with regulatory control in closed loop that was employed in the design of the MPC control is presented next.

Discrete-time BJ model:

Model for output "Y1": y_1(t) $=[B(z) / F(z)] u(t)+$ e_1 $(t)$

$$
\begin{aligned}
& \mathrm{B} 1(z)=z^{\wedge}-1 \\
& \mathrm{~B} 2(z)=0 \\
& \mathrm{~B} 3(z)=0 \\
& \mathrm{~B} 4(z)=0 \\
& \mathrm{~B} 5(z)=0 \\
& \mathrm{~B} 6(z)=0 \\
& \mathrm{~B} 7(z)=0 \\
& \mathrm{~B} 8(z)=0 \\
& \mathrm{~B} 9(z)=0 \\
& \mathrm{~B} 10(z)=0 \\
& \mathrm{~B} 11(z)=0 \\
& \mathrm{~B} 12(z)=0 \\
& \mathrm{~F} 1(z)=1-0.0002263 z^{\wedge}-1 \\
& \mathrm{~F} 2(z)=1 \\
& \mathrm{~F} 3(z)=1 \\
& \mathrm{~F} 4(z)=1 \\
& \mathrm{~F} 5(z)=1 \\
& \mathrm{~F} 6(z)=1 \\
& \mathrm{~F} 7(z)=1 \\
& \mathrm{~F} 8(z)=1 \\
& \mathrm{~F} 9(z)=1 \\
& \mathrm{~F} 10(z)=1 \\
& \mathrm{~F} 11(z)=1 \\
& \mathrm{~F} 12(z)=1
\end{aligned}
$$

Model for output "Y2": y_2(t) $=[B(z) / F(z)] u(t)+e \_2(t)$ 


$$
\begin{aligned}
& \mathrm{B} 1(\mathrm{z})=0 \\
& \mathrm{~B} 2(\mathrm{z})=\mathrm{z}^{\wedge}-1 \\
& \mathrm{~B} 3(\mathrm{z})=0 \\
& \mathrm{~B} 4(\mathrm{z})=0 \\
& \mathrm{~B} 5(\mathrm{z})=0 \\
& \mathrm{~B} 6(\mathrm{z})=0 \\
& \mathrm{~B} 7(\mathrm{z})=0 \\
& \mathrm{~B} 8(\mathrm{z})=0 \\
& \mathrm{~B} 9(\mathrm{z})=0 \\
& \mathrm{~B} 10(\mathrm{z})=0 \\
& \mathrm{~B} 11(\mathrm{z})=0 \\
& \mathrm{~B} 12(\mathrm{z})=0 \\
& \mathrm{~F} 1(\mathrm{z})=1 \\
& \mathrm{~F} 2(\mathrm{z})=1-9.128 \mathrm{e}-07 \mathrm{z}^{\wedge}-1 \\
& \mathrm{~F} 3(\mathrm{z})=1 \\
& \mathrm{~F} 4(\mathrm{z})=1 \\
& \mathrm{~F} 5(\mathrm{z})=1 \\
& \mathrm{~F} 6(\mathrm{z})=1 \\
& \mathrm{~F} 7(\mathrm{z})=1 \\
& \mathrm{~F} 8(\mathrm{z})=1 \\
& \mathrm{~F} 9(\mathrm{z})=1 \\
& \mathrm{~F} 10(\mathrm{z})=1 \\
& \mathrm{~F} 11(\mathrm{z})=1 \\
& \mathrm{~F} 12(\mathrm{z})=1
\end{aligned}
$$

Model for output "Y3": $y \_3(t)=[B(z) / F(z)] u(t)$

$$
\begin{aligned}
& B 1(z)=0 \\
& B 2(z)=0 \\
& B 3(z)=0.9985 z^{\wedge}-1 \\
& B 4(z)=0 \\
& B 5(z)=0 \\
& B 6(z)=0 \\
& B 7(z)=0 \\
& B 8(z)=0
\end{aligned}
$$




$$
\begin{aligned}
& B 9(z)=0 \\
& B 10(z)=0 \\
& B 11(z)=0 \\
& B 12(z)=0 \\
& F 1(z)=1 \\
& F 2(z)=1 \\
& F 3(z)=1-7.276 e-11 z^{\wedge}-1 \\
& F 4(z)=1 \\
& F 5(z)=1 \\
& F 6(z)=1 \\
& F 7(z)=1 \\
& F 8(z)=1 \\
& F 9(z)=1 \\
& F 10(z)=1 \\
& F 11(z)=1 \\
& F 12(z)=1
\end{aligned}
$$

Model for output "Y4": y_4(t) $=[B(z) / F(z)] u(t)$

$$
\begin{aligned}
& \mathrm{B} 1(z)=0 \\
& \mathrm{~B} 2(z)=0 \\
& \mathrm{~B} 3(\mathrm{z})=0 \\
& \mathrm{~B} 4(\mathrm{z})=0.9899 \mathrm{z}^{\wedge}-1 \\
& \mathrm{~B} 5(\mathrm{z})=0 \\
& \mathrm{~B} 6(\mathrm{z})=0 \\
& \mathrm{~B} 7(\mathrm{z})=0 \\
& \mathrm{~B} 8(\mathrm{z})=0 \\
& \mathrm{~B} 9(\mathrm{z})=0 \\
& \mathrm{~B} 10(\mathrm{z})=0 \\
& \mathrm{~B} 11(\mathrm{z})=0 \\
& \mathrm{~B} 12(\mathrm{z})=0 \\
& \mathrm{~F} 1(\mathrm{z})=1 \\
& \mathrm{~F} 2(\mathrm{z})=1 \\
& \mathrm{~F} 3(\mathrm{z})=1 \\
& \mathrm{~F} 4(\mathrm{z})=1-0.008811 \mathrm{z}^{\wedge}-1
\end{aligned}
$$




$$
\begin{aligned}
& F 5(z)=1 \\
& F 6(z)=1 \\
& F 7(z)=1 \\
& F 8(z)=1 \\
& F 9(z)=1 \\
& F 10(z)=1 \\
& F 11(z)=1 \\
& F 12(z)=1
\end{aligned}
$$

Model for output "Y5": $y \_5(t)=[B(z) / F(z)] u(t)+[C(z) / D(z)] e \_5(t)$

$$
\begin{aligned}
& \mathrm{B} 1(\mathrm{z})=0 \\
& \mathrm{~B} 2(\mathrm{z})=-0.0001384 \mathrm{z}^{\wedge}-1 \\
& B 3(z)=-7.488 e-05 z^{\wedge}-1+9.479 e-05 z^{\wedge}-2 \\
& \mathrm{~B} 4(\mathrm{z})=0 \\
& \mathrm{~B} 5(\mathrm{z})=0.0009764 \mathrm{z}^{\wedge}-1-0.001023 \mathrm{z}^{\wedge}-2 \\
& B 6(z)=0.02963 z^{\wedge}-2-0.01607 z^{\wedge}-3-0.01366 z^{\wedge}-4-0.006615 z^{\wedge}-5 \\
& B 7(z)=-0.08753-0.02791 z^{\wedge}-1 \\
& \mathrm{~B} 8(\mathrm{z})=0 \\
& \mathrm{~B} 9(\mathrm{z})=0 \\
& \mathrm{~B} 10(\mathrm{z})=0 \\
& \mathrm{~B} 11(\mathrm{z})=0.007966 \mathrm{z}^{\wedge}-1 \\
& B 12(z)=0.04405 z^{\wedge}-1-0.000332 z^{\wedge}-2+5.32 e-08 z^{\wedge}-3 \\
& C(z)=1-2.186 z^{\wedge}-1+1.458 z^{\wedge}-2-0.2501 z^{\wedge}-3-0.023 z^{\wedge}-4+0.001218 z^{\wedge}-5- \\
& 1.91 e-06 z^{\wedge}-6+9.814 e-10 z^{\wedge}-7+2.585 e-28 z^{\wedge}-8 \\
& D(z)=1-3.273 z^{\wedge}-1+3.933 z^{\wedge}-2-2.06 z^{\wedge}-3+0.4137 z^{\wedge}-4-0.01356 z^{\wedge}-5+ \\
& \text { 2.1e-05 } z^{\wedge}-6-1.073 e-08 z^{\wedge}-7 \\
& \mathrm{~F} 1(\mathrm{z})=1 \\
& \mathrm{~F} 2(\mathrm{z})=1-0.9388 \mathrm{z}^{\wedge}-1 \\
& F 3(z)=1-0.856 z^{\wedge}-1+0.1581 z^{\wedge}-2-0.08499 z^{\wedge}-3+0.181 z^{\wedge}-4-0.00181 z^{\wedge}-5 \\
& \mathrm{~F} 4(\mathrm{z})=1 \\
& \mathrm{~F} 5(\mathrm{z})=1-1.828 \mathrm{z}^{\wedge}-1+0.8288 \mathrm{z}^{\wedge}-2 \\
& F 6(z)=1-0.856 z^{\wedge}-1+0.1581 z^{\wedge}-2-0.08499 z^{\wedge}-3+0.181 z^{\wedge}-4-0.00181 z^{\wedge}-5 \\
& F 7(z)=1-0.4471 z^{\wedge}-1+0.01564 z^{\wedge}-2 \\
& \mathrm{~F} 8(\mathrm{z})=1
\end{aligned}
$$


$\mathrm{F} 9(\mathrm{z})=1$

$\mathrm{F} 10(\mathrm{z})=1$

$F 11(z)=1-0.856 z^{\wedge}-1+0.1581 z^{\wedge}-2-0.08499 z^{\wedge}-3+0.18 z^{\wedge}-4-0.0018 z^{\wedge}-5$

$F 12(z)=1-0.001601 z^{\wedge}-1+8.313 e-07 z^{\wedge}-2$

Model for output "Y6": $y \_6(t)=[B(z) / F(z)] u(t)+[C(z) / D(z)] e \_6(t)$

$\mathrm{B} 1(\mathrm{z})=0$

$B 2(z)=-1.389 e-05 z^{\wedge}-1-1.869 e-05 z^{\wedge}-2$

$\mathrm{B} 3(\mathrm{z})=-1.849 \mathrm{e}-06 \mathrm{z}^{\wedge}-1+1.209 \mathrm{e}-06 \mathrm{z}^{\wedge}-2$

$B 4(z)=0.0003997 z^{\wedge}-1-0.0002613 z^{\wedge}-2$

$B 5(z)=1.979 e-06 z^{\wedge}-1-0.0005496 z^{\wedge}-2$

$\mathrm{B} 6(\mathrm{z})=0$

$B 7(z)=0.02934 z^{\wedge}-1-0.04329 z^{\wedge}-2$

$\mathrm{B} 8(\mathrm{z})=0$

$\mathrm{B} 9(\mathrm{z})=0$

$\mathrm{B} 10(\mathrm{z})=0$

$B 11(z)=-0.0005079 z^{\wedge}-1+0.000332 z^{\wedge}-2$

$B 12(z)=-0.02354 z^{\wedge}-1+0.02678 z^{\wedge}-2$

$C(z)=1+0.9981 z^{\wedge}-1$

$D(z)=1-0.9962 z^{\wedge}-1$

$\mathrm{F} 1(\mathrm{z})=1$

$F 2(z)=1-1.241 z^{\wedge}-1+0.2412 z^{\wedge}-2$

$F 3(z)=1-1.945 z^{\wedge}-1+0.945 z^{\wedge}-2$

$F 4(z)=1-1.945 z^{\wedge}-1+0.945 z^{\wedge}-2$

$\mathrm{F} 5(\mathrm{z})=1-\mathrm{z}^{\wedge}-1$

$\mathrm{F} 6(\mathrm{z})=1$

$F 7(z)=1-0.9472 z^{\wedge}-1$

$F 8(z)=1$

$\mathrm{F} 9(\mathrm{z})=1$

$\mathrm{F} 10(\mathrm{z})=1$

$F 11(z)=1-1.945 z^{\wedge}-1+0.945 z^{\wedge}-2$

$F 12(z)=1-0.9985 z^{\wedge}-1$

Model for output "Y7": $y \_7(t)=[B(z) / F(z)] u(t)+[1 / D(z)] e \_7(t)$

$\mathrm{B} 1(\mathrm{z})=8.732 \mathrm{z}^{\wedge}-1$ 


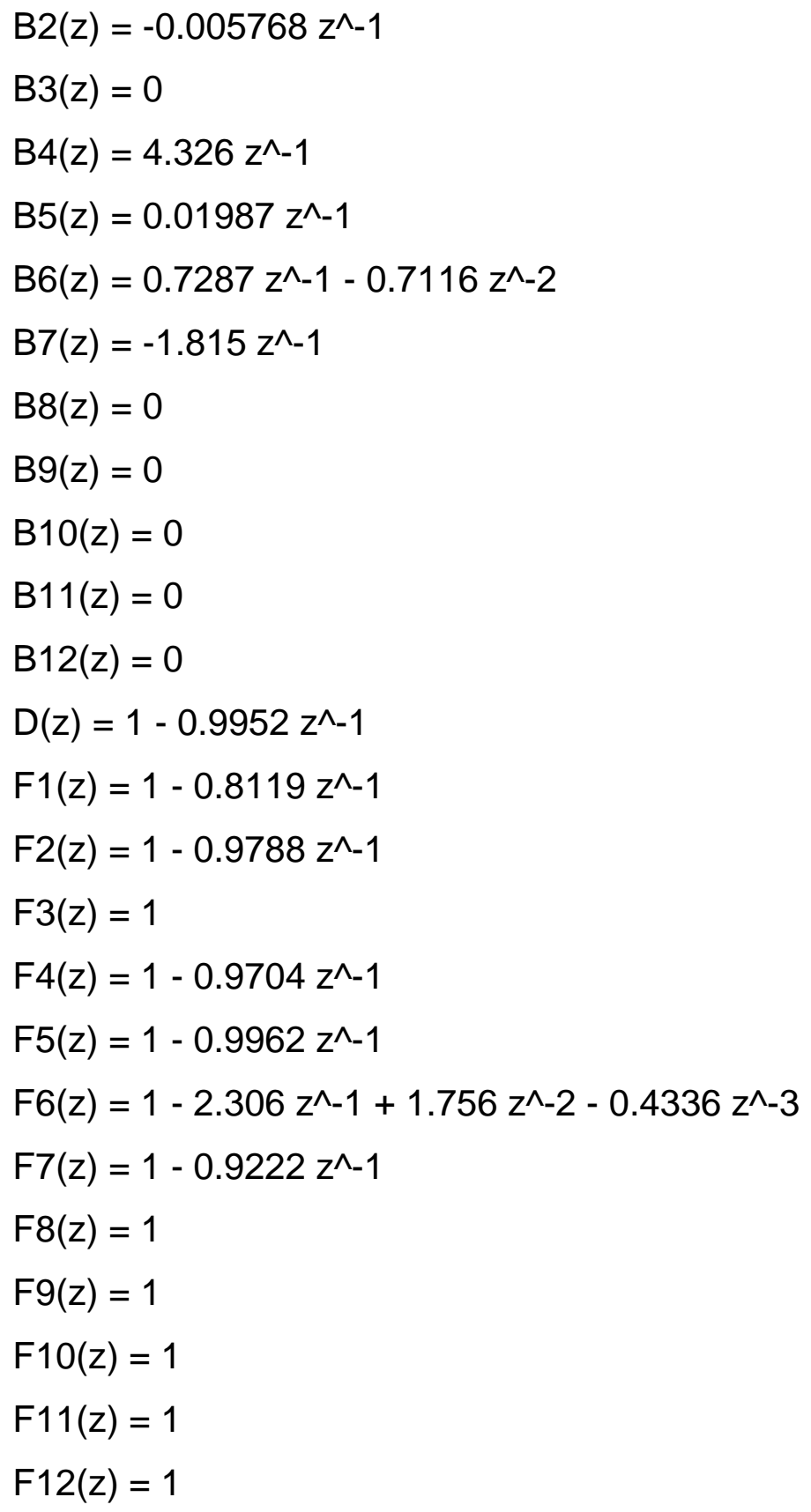

Model for output "Y8": $y \_8(t)=[B(z) / F(z)] u(t)+e \_8(t)$

$B 1(z)=-0.001399 z^{\wedge}-1$

$B 2(z)=0.0001458 z^{\wedge}-1-5.274 e-05 z^{\wedge}-2$

$\mathrm{B} 3(\mathrm{z})=0$

$B 4(z)=0.1603 z^{\wedge}-17$

$\mathrm{B} 5(\mathrm{z})=0$

$B 6(z)=0.002618 z^{\wedge}-1+0.02577 z^{\wedge}-2$

$B 7(z)=0.05836 z^{\wedge}-1-0.1261 z^{\wedge}-2+0.06767 z^{\wedge}-3$

$B 8(z)=0$ 


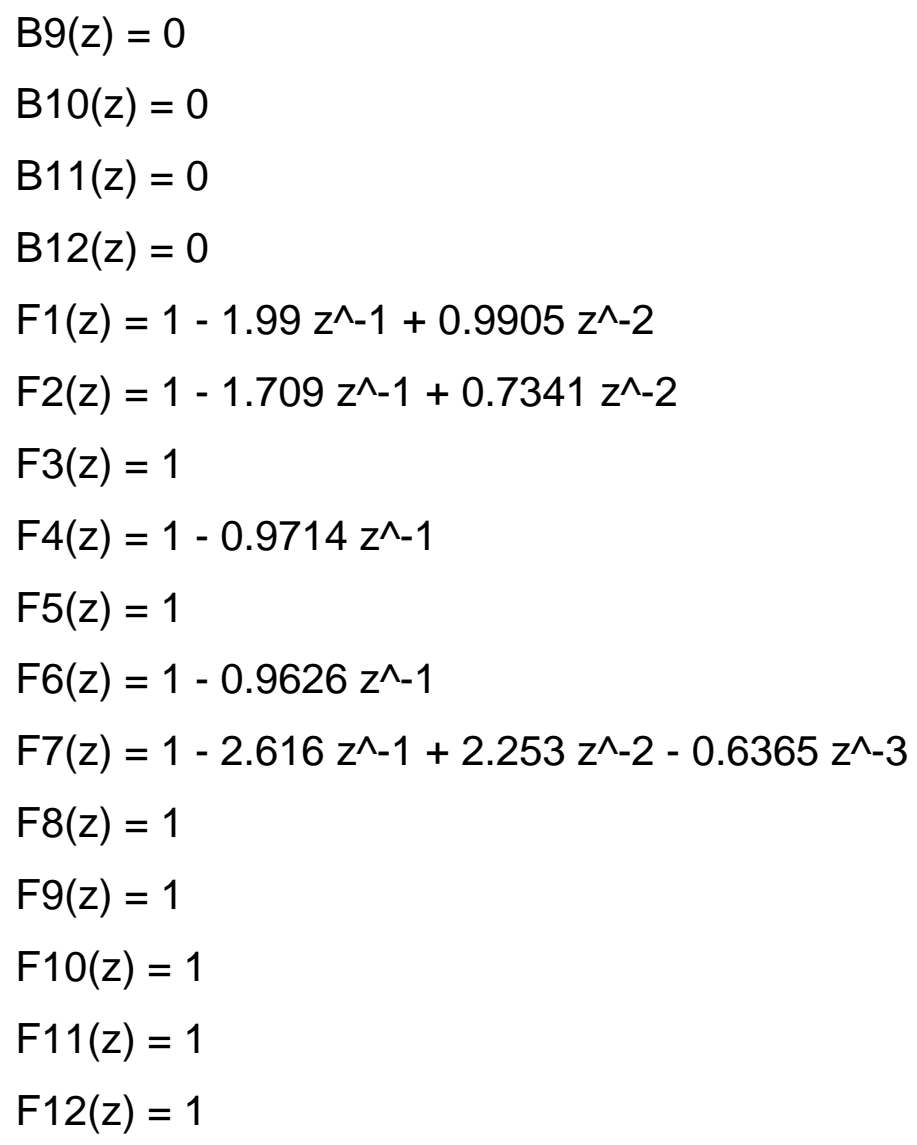

Model for output "Y9": y_9(t) = [B(z)/F(z)]u(t) $+[\mathrm{C}(\mathrm{z}) / \mathrm{D}(\mathrm{z})] \mathrm{e} \_9(\mathrm{t})$

$\mathrm{B} 1(\mathrm{z})=0$

$\mathrm{B} 2(\mathrm{z})=0.0001825 z^{\wedge}-1-0.0001832 z^{\wedge}-2$

$\mathrm{B} 3(\mathrm{z})=0$

$\mathrm{B} 4(\mathrm{z})=0$

$\mathrm{B} 5(\mathrm{z})=0$

$B 6(z)=0.002784 z^{\wedge}-1-0.002944 z^{\wedge}-2$

$B 7(z)=0.428 z^{\wedge}-1$

$\mathrm{B} 8(\mathrm{z})=0$

$\mathrm{B} 9(\mathrm{z})=0$

$\mathrm{B} 10(\mathrm{z})=0$

$\mathrm{B} 11(\mathrm{z})=0$

$B 12(z)=0$

$C(z)=1-1.332 z^{\wedge}-1-0.04689 z^{\wedge}-2+0.4438 z^{\wedge}-3$

$D(z)=1-2.818 z^{\wedge}-1+2.683 z^{\wedge}-2-0.8637 z^{\wedge}-3$

$\mathrm{F} 1(\mathrm{z})=1$

$F 2(z)=1-1.382 z^{\wedge}-1+0.4676 z^{\wedge}-2$ 


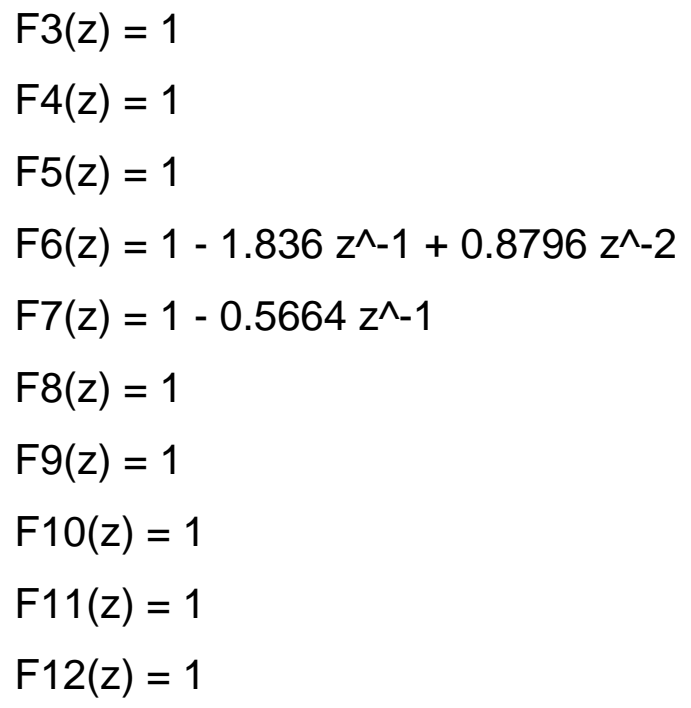

Model for output "Y10": $y \_10(t)=[B(z) / F(z)] u(t)+[C(z) / D(z)] e \_10(t)$

$\mathrm{B} 1(\mathrm{z})=0$

$B 2(z)=-1.395 e-05 z^{\wedge}-1$

$\mathrm{B} 3(\mathrm{z})=0$

$B 4(z)=0.05728 z^{\wedge}-1$

$\mathrm{B} 5(\mathrm{z})=0$

$B 6(z)=0.003655+0.001413 z^{\wedge}-1$

$B 7(z)=-0.003761 z^{\wedge}-1$

$\mathrm{B} 8(\mathrm{z})=0$

$\mathrm{B} 9(\mathrm{z})=0$

$\mathrm{B} 10(\mathrm{z})=0$

$\mathrm{B} 11(\mathrm{z})=0$

$\mathrm{B} 12(\mathrm{z})=0$

$C(z)=1-0.09733 z^{\wedge}-1-0.3036 z^{\wedge}-2$

$D(z)=1-1.584 z^{\wedge}-1+0.5912 z^{\wedge}-2$

$\mathrm{F} 1(\mathrm{z})=1$

$F 2(z)=1-0.9706 z^{\wedge}-1$

$\mathrm{F} 3(\mathrm{z})=1$

$F 4(z)=1-0.9209 z^{\wedge}-1$

$\mathrm{F} 5(\mathrm{z})=1$

$\mathrm{F} 6(\mathrm{z})=1-0.6018 \mathrm{z}^{\wedge}-1$

$F 7(z)=1-0.9514 z^{\wedge}-1$

$F 8(z)=1$ 


$$
\begin{aligned}
& F 9(z)=1 \\
& F 10(z)=1 \\
& F 11(z)=1 \\
& F 12(z)=1
\end{aligned}
$$

Model for output "Y11": y_11(t) = [B(z)/F(z)]u(t)

$$
\begin{aligned}
& \mathrm{B} 1(\mathrm{z})=0 \\
& \mathrm{~B} 2(\mathrm{z})=-0.0002645 \mathrm{z}^{\wedge}-1 \\
& \mathrm{~B} 3(\mathrm{z})=0 \\
& \mathrm{~B} 4(\mathrm{z})=0 \\
& \mathrm{~B} 5(\mathrm{z})=0 \\
& B 6(z)=0.1082 z^{\wedge}-1-0.2198 z^{\wedge}-2+0.1249 z^{\wedge}-3-0.0135 z^{\wedge}-4 \\
& B 7(z)=0.072 z^{\wedge}-1 \\
& \mathrm{~B} 8(\mathrm{z})=0.01046 \mathrm{z}^{\wedge}-1 \\
& \mathrm{~B} 9(\mathrm{z})=0 \\
& \mathrm{~B} 10(\mathrm{z})=0 \\
& B 11(z)=0 \\
& \mathrm{~B} 12(\mathrm{z})=-0.03065 \mathrm{z}^{\wedge}-1+0.01402 \mathrm{z}^{\wedge}-2 \\
& D(z)=1-0.9513 z^{\wedge}-1 \\
& \mathrm{~F} 1(\mathrm{z})=1 \\
& F 2(z)=1-0.9359 z^{\wedge}-1 \\
& \mathrm{~F} 3(\mathrm{z})=1 \\
& \mathrm{~F} 4(\mathrm{z})=1 \\
& \mathrm{~F} 5(\mathrm{z})=1 \\
& \mathrm{~F} 6(z)=1-3.189 z^{\wedge}-1+3.753 z^{\wedge}-2-1.913 z^{\wedge}-3+0.3495 z^{\wedge}-4 \\
& \mathrm{~F} 7(\mathrm{z})=1-0.9608 \mathrm{z}^{\wedge}-1 \\
& F 8(z)=1-0.9408 z^{\wedge}-1 \\
& \mathrm{~F} 9(\mathrm{z})=1 \\
& \mathrm{~F} 10(\mathrm{z})=1 \\
& \mathrm{~F} 11(\mathrm{z})=1 \\
& F 12(z)=1-1.742 z^{\wedge}-1+0.7643 z^{\wedge}-2
\end{aligned}
$$

Model for output "Y12": y_12(t) = [B(z)/F(z)]u(t) + e_12(t)

$$
\begin{aligned}
& \mathrm{B} 1(\mathrm{z})=0 \\
& \mathrm{~B} 2(\mathrm{z})=0
\end{aligned}
$$




$$
\begin{aligned}
& \mathrm{B} 3(\mathrm{z})=0 \\
& B 4(z)=0 \\
& \mathrm{~B} 5(\mathrm{z})=0 \\
& B 6(z)=0 \\
& \mathrm{~B} 7(\mathrm{z})=0 \\
& B 8(z)=0.2862 z^{\wedge}-1 \\
& \mathrm{~B} 9(\mathrm{z})=0 \\
& \mathrm{~B} 10(\mathrm{z})=0 \\
& \mathrm{~B} 11(\mathrm{z})=0 \\
& \mathrm{~B} 12(\mathrm{z})=0 \\
& \mathrm{~F} 1(\mathrm{z})=1 \\
& \mathrm{~F} 2(\mathrm{z})=1 \\
& \mathrm{~F} 3(\mathrm{z})=1 \\
& \mathrm{~F} 4(\mathrm{z})=1 \\
& \mathrm{~F} 5(\mathrm{z})=1 \\
& \mathrm{~F} 6(\mathrm{z})=1 \\
& F 7(z)=1 \\
& F 8(z)=1-0.7101 z^{\wedge}-1 \\
& \mathrm{~F} 9(\mathrm{z})=1 \\
& \mathrm{~F} 10(\mathrm{z})=1 \\
& \mathrm{~F} 11(\mathrm{z})=1 \\
& F 12(z)=1
\end{aligned}
$$

Model for output "Y13": $y \_13(t)=[B(z) / F(z)] u(t)+[C(z) / D(z)] e \_13(t)$

$$
\begin{aligned}
& \text { B1 }(z)=0 \\
& \text { B2(z) }=-0.00609 z^{\wedge}-1 \\
& \text { B3(z) }=0 \\
& \text { B4(z) }=1.04 z^{\wedge}-3 \\
& \text { B5(z) }=0 \\
& \text { B6 (z) }=0.6007 z^{\wedge-1} \\
& \text { B7(z) }=-1.434 z^{\wedge}-1 \\
& \text { B8(z) }=0 \\
& \text { B9(z) }=0 \\
& \text { B10(z) }=0
\end{aligned}
$$




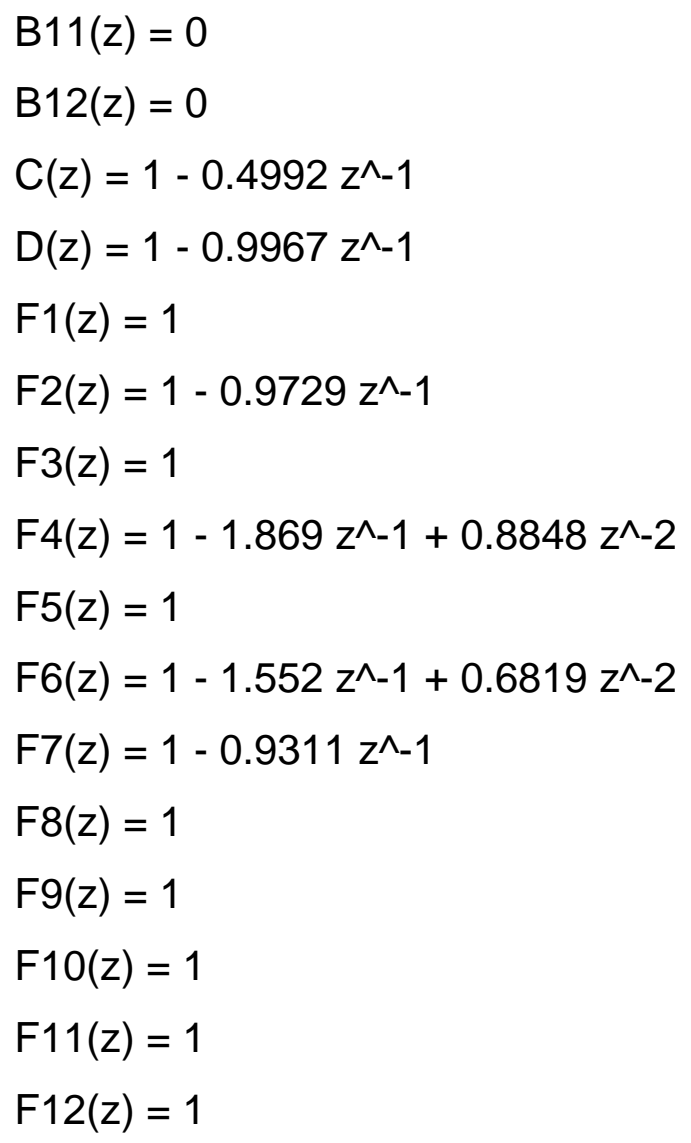

Model for output "Y14": $y_{-} 14(\mathrm{t})=[\mathrm{B}(\mathrm{z}) / \mathrm{F}(\mathrm{z})] \mathrm{u}(\mathrm{t})+[\mathrm{C}(\mathrm{z}) / \mathrm{D}(\mathrm{z})] \mathrm{e} \_14(\mathrm{t})$

$\mathrm{B} 1(\mathrm{z})=0$

$B 2(z)=9.005 e-05 z^{\wedge}-1$

$\mathrm{B} 3(\mathrm{z})=0$

$B 4(z)=-0.1738 z^{\wedge}-26+0.1575 z^{\wedge}-27$

$\mathrm{B} 5(\mathrm{z})=0$

$\mathrm{B} 6(\mathrm{z})=-0.01443 z^{\wedge}-1-0.003173 z^{\wedge}-2-0.005957 z^{\wedge}-3+0.007196 z^{\wedge}-4+$ $0.01201 z^{\wedge}-5+0.004612 z^{\wedge}-6$

$B 7(z)=0.1428 z^{\wedge}-1-0.01932 z^{\wedge}-2-0.1256 z^{\wedge}-3$

$B 8(z)=-1.214 z^{\wedge}-1+1.092 z^{\wedge}-2+0.1307 z^{\wedge}-3$

$\mathrm{B} 9(\mathrm{z})=0$

$\mathrm{B} 10(\mathrm{z})=0$

$\mathrm{B} 11(\mathrm{z})=0$

$B 12(z)=0$

$C(z)=1+0.9779 z^{\wedge}-1$

$\mathrm{D}(\mathrm{z})=1$

$\mathrm{F} 1(\mathrm{z})=1$ 


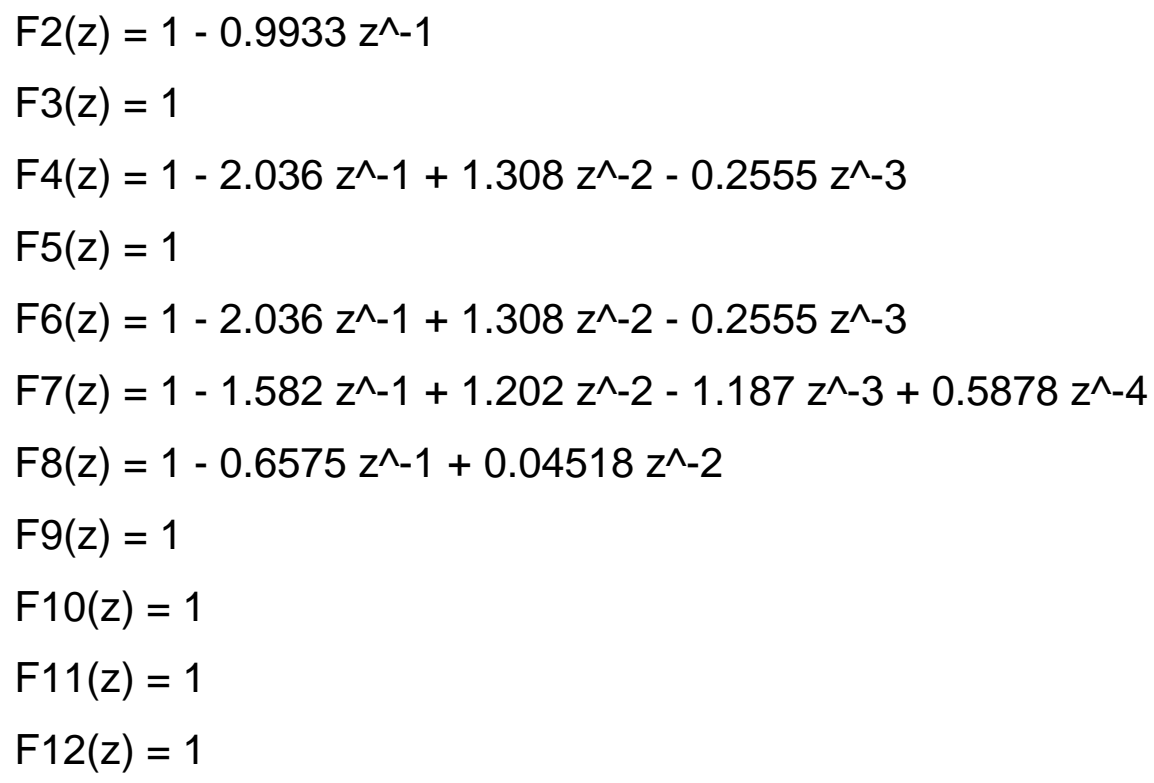

Model for output "Y15": $y \_15(t)=[B(z) / F(z)] u(t)+e \_15(t)$

$B 1(z)=0$

$\mathrm{B} 2(\mathrm{z})=0$

$\mathrm{B} 3(\mathrm{z})=0$

$B 4(z)=0$

$\mathrm{B} 5(\mathrm{z})=0$

$\mathrm{B} 6(\mathrm{z})=0.1015 \mathrm{z}^{\wedge}-1-0.2766 \mathrm{z}^{\wedge}-2+0.1762 \mathrm{z}^{\wedge}-3$

$B 7(z)=0.09022 z^{\wedge}-1-0.09131 z^{\wedge}-2$

$B 8(z)=-0.4159 z^{\wedge}-1+0.8318 z^{\wedge}-2-0.4158 z^{\wedge}-3$

$B 9(z)=0.2725 z^{\wedge}-1-0.2269 z^{\wedge}-2$

$\mathrm{B} 10(\mathrm{z})=0$

$\mathrm{B} 11(\mathrm{z})=0$

$\mathrm{B} 12(\mathrm{z})=0$

$\mathrm{F} 1(\mathrm{z})=1$

$\mathrm{F} 2(\mathrm{z})=1$

$\mathrm{F} 3(\mathrm{z})=1$

$\mathrm{F} 4(\mathrm{z})=1$

$\mathrm{F} 5(\mathrm{z})=1$

$\mathrm{F} 6(\mathrm{z})=1-1.854 \mathrm{z}^{\wedge}-1+0.8838 \mathrm{z}^{\wedge}-2$

$F 7(z)=1-1.834 z^{\wedge}-1+0.8538 z^{\wedge}-2$

$F 8(z)=1-2.362 z^{\wedge}-1+1.854 z^{\wedge}-2-0.4817 z^{\wedge}-3$

$F 9(z)=1-1.615 z^{\wedge}-1+0.5948 z^{\wedge}-2+0.06599 z^{\wedge}-3$ 


$$
\begin{aligned}
& F 10(z)=1 \\
& F 11(z)=1 \\
& F 12(z)=1
\end{aligned}
$$

Model for output "Y16": y_16(t) = [B(z)/F(z)]u(t) + [C(z)/D(z)]e_16(t)

$$
\begin{aligned}
& B 1(z)=9.971 z^{\wedge}-1 \\
& B 2(z)=-0.007481 z^{\wedge}-1+0.00739 z^{\wedge}-2 \\
& \mathrm{~B} 3(\mathrm{z})=0 \\
& B 4(z)=5.276 z^{\wedge}-1 \\
& \mathrm{~B} 5(\mathrm{z})=0.01379 \mathrm{z}^{\wedge}-1 \\
& B 6(z)=0.7355 z^{\wedge}-1-0.714 z^{\wedge}-2 \\
& B 7(z)=-1.638 z^{\wedge}-1 \\
& \mathrm{~B} 8(\mathrm{z})=0 \\
& \mathrm{~B} 9(\mathrm{z})=0 \\
& \mathrm{~B} 10(\mathrm{z})=0 \\
& \mathrm{~B} 11(\mathrm{z})=0 \\
& \mathrm{~B} 12(\mathrm{z})=-0.5922 \mathrm{z}^{\wedge}-1 \\
& C(z)=1-0.7941 z^{\wedge}-1 \\
& \mathrm{D}(\mathrm{z})=1-0.9855 \mathrm{z}^{\wedge}-1 \\
& \mathrm{~F} 1(\mathrm{z})=1-0.8342 \mathrm{z}^{\wedge}-1 \\
& F 2(z)=1-1.956 z^{\wedge}-1+0.9563 z^{\wedge}-2 \\
& \mathrm{~F} 3(\mathrm{z})=1 \\
& \mathrm{~F} 4(\mathrm{z})=1-0.9651 \mathrm{z}^{\wedge}-1 \\
& \mathrm{~F} 5(\mathrm{z})=1-0.998 \mathrm{z}^{\wedge}-1 \\
& F 6(z)=1-2.36 z^{\wedge}-1+1.849 z^{\wedge}-2-0.4739 z^{\wedge}-3 \\
& \mathrm{~F} 7(\mathrm{z})=1-0.9156 \mathrm{z}^{\wedge}-1 \\
& \mathrm{~F} 8(\mathrm{z})=1 \\
& \mathrm{~F} 9(\mathrm{z})=1 \\
& \mathrm{~F} 10(\mathrm{z})=1 \\
& \mathrm{~F} 11(\mathrm{z})=1 \\
& \mathrm{~F} 12(\mathrm{z})=1-0.8457 \mathrm{z}^{\wedge}-1
\end{aligned}
$$

Model for output "Y17": y_17(t) = [B(z)/F(z)]u(t) + [C(z)/D(z)]e_17(t)

$$
\begin{aligned}
& B 1(z)=0 \\
& B 2(z)=-0.0004049 z^{\wedge}-1+0.0004454 z^{\wedge}-2
\end{aligned}
$$




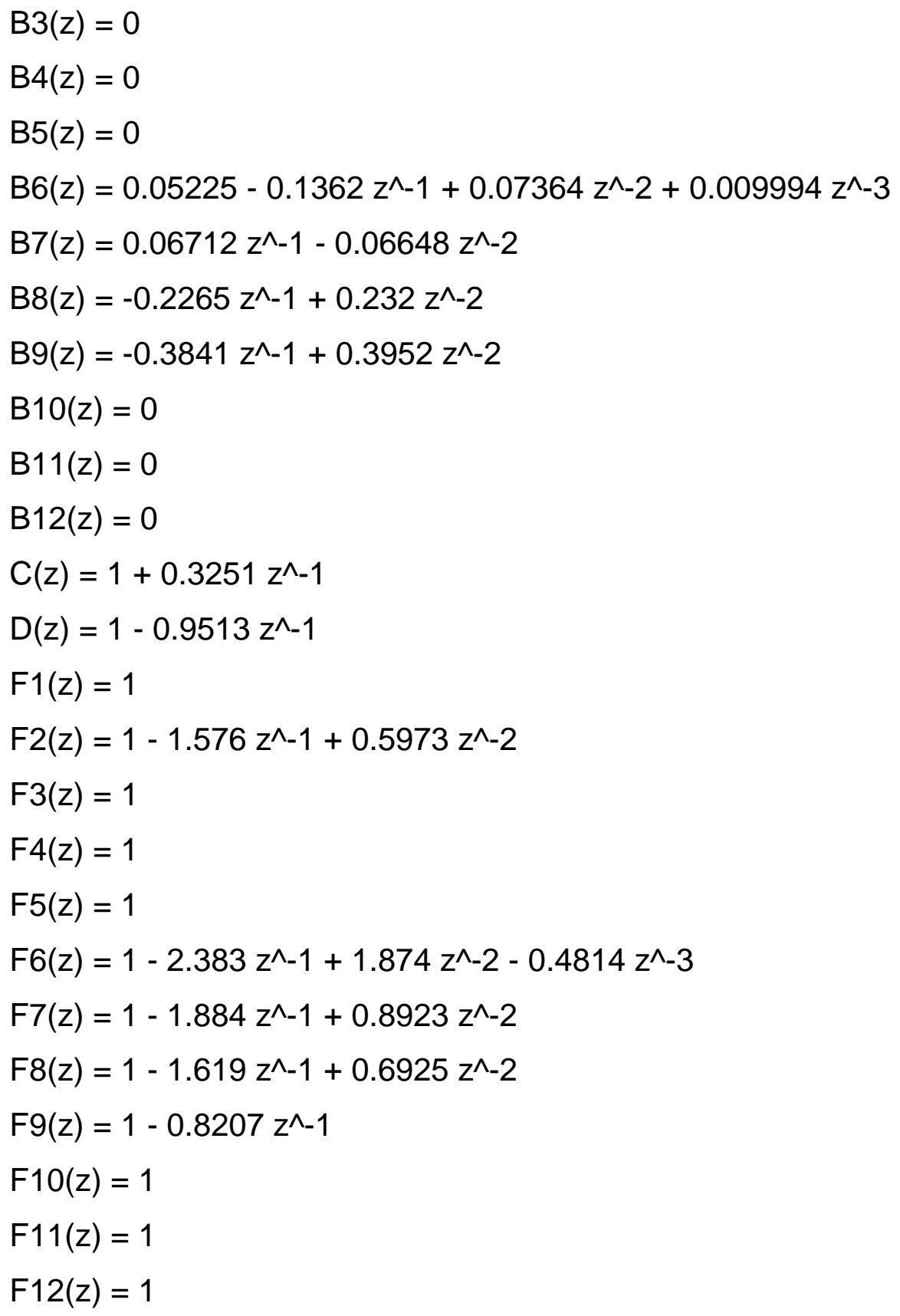

Model for output "Y18": y_18(t) = [B(z)/F(z)]u(t) + [C(z)/D(z)]e_18(t)

$\mathrm{B} 1(\mathrm{z})=0$

$\mathrm{B} 2(\mathrm{z})=0$

$\mathrm{B} 3(\mathrm{z})=0$

$\mathrm{B} 4(\mathrm{z})=0$

$\mathrm{B} 5(\mathrm{z})=0$

$B 6(z)=0.009638 z^{\wedge}-1+0.0001477 z^{\wedge}-2-0.009763 z^{\wedge}-3$

$B 7(z)=0.001772 z^{\wedge}-6$

$B 8(z)=0.0006325 z^{\wedge}-22-0.0006315 z^{\wedge}-23$ 


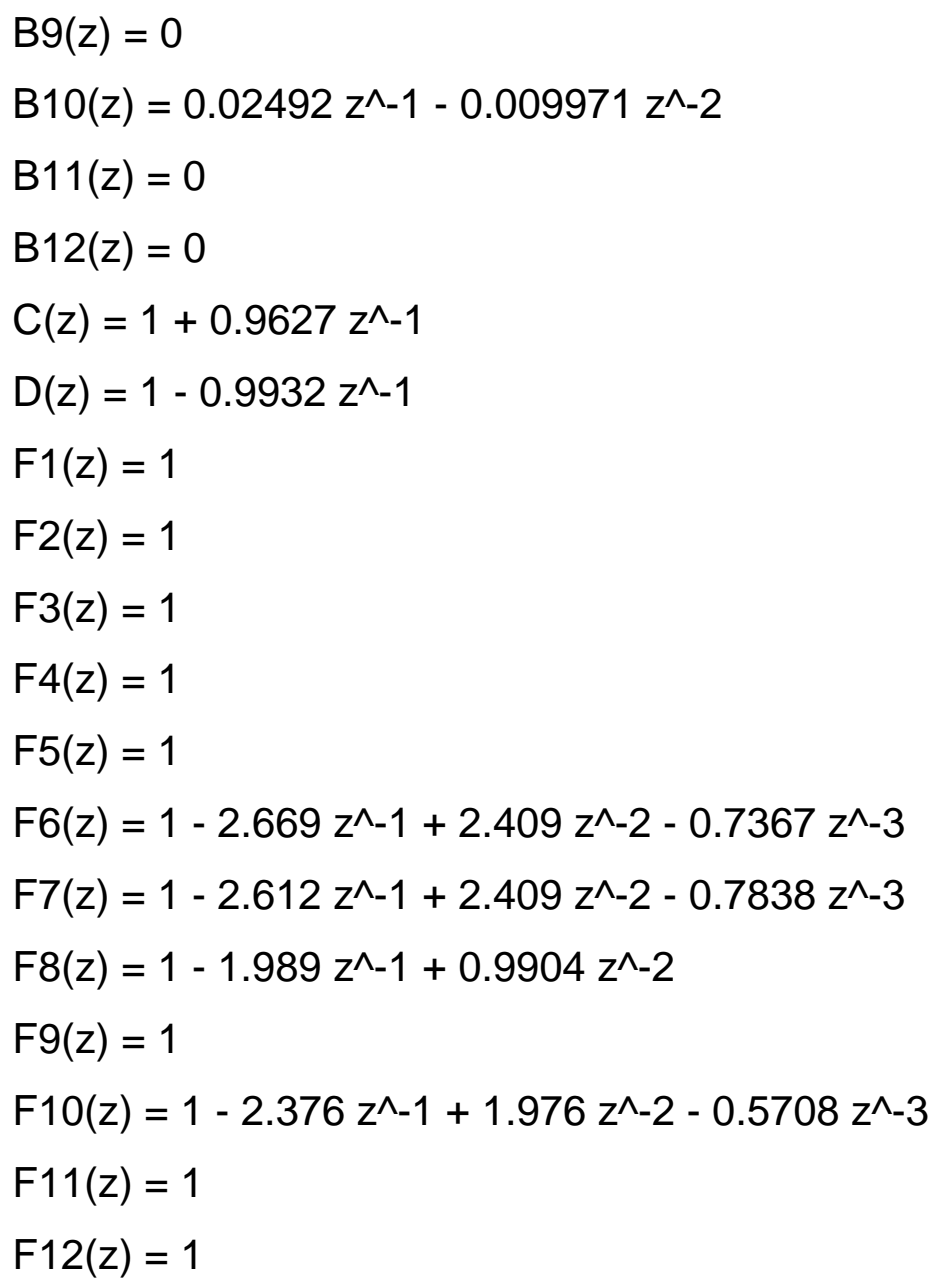

Model for output "Y19": y_19(t) = [B(z)/F(z)]u(t) + [C(z)/D(z)]e_19(t)

$\mathrm{B} 1(\mathrm{z})=0$

$B 2(z)=-0.0001786+0.0007679 z^{\wedge}-1-0.0007538 z^{\wedge}-2+0.0001726 z^{\wedge}-3$

$\mathrm{B} 3(\mathrm{z})=0$

$B 4(z)=9.267-2.344 z^{\wedge}-1-8.277 z^{\wedge}-2$

$\mathrm{B} 5(\mathrm{z})=0$

$B 6(z)=-1.516 z^{\wedge}-1+1.524 z^{\wedge}-2$

$B 7(z)=0.1006-0.2602 z^{\wedge}-1-0.1246 z^{\wedge}-2$

$B 8(z)=-0.02704+0.1578 z^{\wedge}-1-0.1523 z^{\wedge}-2-0.233 z^{\wedge}-3$

$\mathrm{B} 9(\mathrm{z})=0$

$\mathrm{B} 10(\mathrm{z})=10.62 \mathrm{z}^{\wedge}-1-1.67 \mathrm{z}^{\wedge}-2$

$\mathrm{B} 11(\mathrm{z})=0$

$\mathrm{B} 12(\mathrm{z})=0$

$\mathrm{C}(\mathrm{z})=1-0.3333 \mathrm{z}^{\wedge}-1$

$D(z)=1-0.9839 z^{\wedge}-1$ 


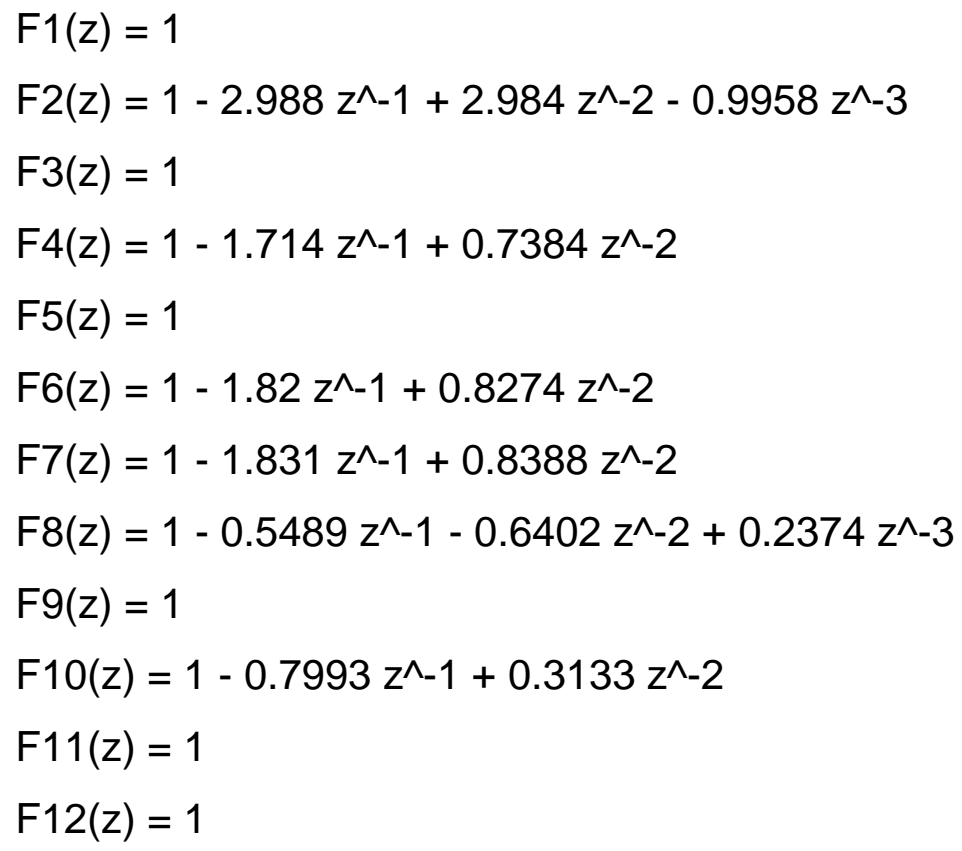

Model for output "Y20": $y \_20(t)=[B(z) / F(z)] u(t)+[C(z) / D(z)] e \_20(t)$

$$
\begin{aligned}
& B 1(z)=0 \\
& B 2(z)=0 \\
& B 3(z)=-0.0003163 z^{\wedge}-1 \\
& B 4(z)=0 \\
& B 5(z)=0.001588 z^{\wedge}-1 \\
& B 6(z)=-0.03177 z^{\wedge}-1+0.02659 z^{\wedge}-2 \\
& B 7(z)=-0.06497 z^{\wedge}-1-0.02553 z^{\wedge}-2 \\
& B 8(z)=0.01491 z^{\wedge}-1-0.01077 z^{\wedge}-2 \\
& B 9(z)=0 \\
& B 10(z)=0 \\
& B 11(z)=0 \\
& B 12(z)=0.4467 z^{\wedge}-1-0.1426 z^{\wedge}-2+1.979 e-18 z^{\wedge}-3 \\
& C(z)=1+0.8573 z^{\wedge}-1 \\
& \text { D }(z)=1-z^{\wedge}-1 \\
& \text { F1 (z) }=1 \\
& \text { F2 }(z)=1 \\
& \text { F3 }(z)=1-z^{\wedge}-1 \\
& \text { F4(z) }=1 \\
& \text { F5 (z) }=1-0.9623 z^{\wedge}-1 \\
& \text { F6 (z) }=1-1.88 z^{\wedge}-1+0.9239 z^{\wedge}-2
\end{aligned}
$$




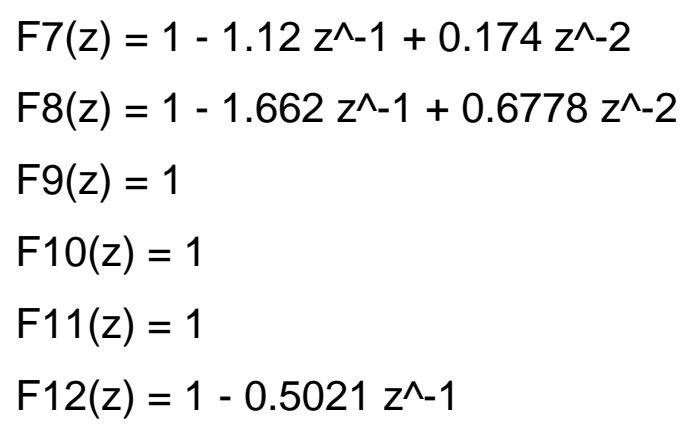

Model for output "Y21": y_21(t) $=[B(z) / F(z)] u(t)+C(z) e \_21(t)$

$$
\begin{aligned}
& \mathrm{B} 1(\mathrm{z})=0 \\
& \mathrm{~B} 2(\mathrm{z})=0 \\
& \mathrm{~B} 3(\mathrm{z})=0 \\
& \mathrm{~B} 4(\mathrm{z})=0 \\
& \mathrm{~B} 5(\mathrm{z})=0 \\
& \mathrm{~B} 6(\mathrm{z})=0 \\
& B 7(z)=0.5132 z^{\wedge}-1-0.507 z^{\wedge}-2 \\
& \mathrm{~B} 8(\mathrm{z})=0 \\
& \mathrm{~B} 9(\mathrm{z})=0 \\
& \mathrm{~B} 10(\mathrm{z})=0 \\
& B 11(z)=0.04311 z^{\wedge}-1 \\
& \mathrm{~B} 12(\mathrm{z})=0 \\
& C(z)=1 \\
& \mathrm{~F} 1(\mathrm{z})=1 \\
& \mathrm{~F} 2(\mathrm{z})=1 \\
& \mathrm{~F} 3(\mathrm{z})=1 \\
& F 4(z)=1 \\
& \mathrm{~F} 5(\mathrm{z})=1 \\
& \mathrm{~F} 6(\mathrm{z})=1 \\
& F 7(z)=1-0.9406 z^{\wedge}-1+4.082 e-05 z^{\wedge}-2 \\
& \mathrm{~F} 8(\mathrm{z})=1 \\
& \mathrm{~F} 9(\mathrm{z})=1 \\
& \mathrm{~F} 10(\mathrm{z})=1 \\
& \mathrm{~F} 11(\mathrm{z})=1-0.9539 z^{\wedge}-1 \\
& \mathrm{~F} 12(\mathrm{z})=1
\end{aligned}
$$

Model for output "Y22": y_22(t) = [B(z)/F(z)]u(t) + [C(z)/D(z)]e_22(t) 


$$
\begin{aligned}
& \mathrm{B} 1(\mathrm{z})=0 \\
& B 2(z)=-0.0004026 z^{\wedge}-1 \\
& \mathrm{~B} 3(\mathrm{z})=-8.36 \mathrm{e}-06 \mathrm{z}^{\wedge}-1 \\
& B 4(z)=-0.47 z^{\wedge}-1+0.5893 z^{\wedge}-2+0.4327 z^{\wedge}-3-0.5484 z^{\wedge}-4 \\
& \mathrm{~B} 5(\mathrm{z})=0.001714 \mathrm{z}^{\wedge}-1 \\
& B 6(z)=0.1742 z^{\wedge}-1-0.303 z^{\wedge}-2+0.08049 z^{\wedge}-3+0.04825 z^{\wedge}-4 \\
& B 7(z)=0.02748 z^{\wedge}-1 \\
& \mathrm{~B} 8(\mathrm{z})=0.004091 \mathrm{z}^{\wedge}-1 \\
& \mathrm{~B} 9(\mathrm{z})=0.0001161 \mathrm{z}^{\wedge}-1 \\
& B 10(z)=-0.002702 z^{\wedge}-1+0.002629 z^{\wedge}-2 \\
& B 11(z)=0.04227 z^{\wedge}-1-0.06346 z^{\wedge}-2+0.00416 z^{\wedge}-3+0.017 z^{\wedge}-4 \\
& B 12(z)=-0.01031 z^{\wedge}-1 \\
& C(z)=1-0.7952 z^{\wedge}-1 \\
& \mathrm{D}(\mathrm{z})=1-0.9882 \mathrm{z}^{\wedge}-1 \\
& \mathrm{~F} 1(\mathrm{z})=1 \\
& \mathrm{~F} 2(\mathrm{z})=1-0.9882 \mathrm{z}^{\wedge}-1 \\
& F 3(z)=1-2.67 z^{\wedge}-1+2.467 z^{\wedge}-2-0.8831 z^{\wedge}-3+0.08611 z^{\wedge}-4 \\
& F 4(z)=1-2.67 z^{\wedge}-1+2.467 z^{\wedge}-2-0.8831 z^{\wedge}-3+0.08611 z^{\wedge}-4 \\
& \mathrm{~F} 5(\mathrm{z})=1-0.9882 \mathrm{z}^{\wedge}-1 \\
& F 6(z)=1-2.67 z^{\wedge}-1+2.467 z^{\wedge}-2-0.8831 z^{\wedge}-3+0.08611 z^{\wedge}-4 \\
& F 7(z)=1-0.9882 z^{\wedge}-1 \\
& \mathrm{~F} 8(\mathrm{z})=1-0.9882 \mathrm{z}^{\wedge}-1 \\
& F 9(z)=1-2.67 z^{\wedge}-1+2.467 z^{\wedge}-2-0.8831 z^{\wedge}-3+0.08611 z^{\wedge}-4 \\
& F 10(z)=1-2.67 z^{\wedge}-1+2.467 z^{\wedge}-2-0.8831 z^{\wedge}-3+0.08611 z^{\wedge}-4 \\
& F 11(z)=1-2.67 z^{\wedge}-1+2.467 z^{\wedge}-2-0.8831 z^{\wedge}-3+0.08611 z^{\wedge}-4 \\
& F 12(z)=1-0.9882 z^{\wedge}-1
\end{aligned}
$$

Sample time: 1 minute

Polynomial orders:

$\mathrm{nb}=$

$\begin{array}{llllllllllll}{[1} & 0 & 0 & 0 & 0 & 0 & 0 & 0 & 0 & 0 & 0 & 0 ; \\ 0 & 1 & 0 & 0 & 0 & 0 & 0 & 0 & 0 & 0 & 0 & 0 ; \\ 0 & 0 & 1 & 0 & 0 & 0 & 0 & 0 & 0 & 0 & 0 & 0 ;\end{array}$




$\begin{array}{llllllllllll}0 & 0 & 0 & 1 & 0 & 0 & 0 & 0 & 0 & 0 & 0 & 0 ; \\ 0 & 1 & 2 & 0 & 2 & 4 & 3 & 0 & 0 & 0 & 1 & 3 ; \\ 0 & 2 & 2 & 2 & 2 & 0 & 2 & 0 & 0 & 0 & 2 & 2 ; \\ 1 & 1 & 0 & 1 & 1 & 2 & 1 & 0 & 0 & 0 & 0 & 0 ; \\ 1 & 2 & 0 & 1 & 0 & 2 & 3 & 0 & 0 & 0 & 0 & 0 ; \\ 0 & 2 & 0 & 0 & 0 & 2 & 1 & 0 & 0 & 0 & 0 & 0 ; \\ 0 & 1 & 0 & 1 & 0 & 2 & 1 & 0 & 0 & 0 & 0 & 0 ; \\ 0 & 1 & 0 & 0 & 0 & 4 & 1 & 1 & 0 & 0 & 0 & 2 ; \\ 0 & 0 & 0 & 0 & 0 & 0 & 0 & 1 & 0 & 0 & 0 & 0 ; \\ 0 & 1 & 0 & 1 & 0 & 1 & 1 & 0 & 0 & 0 & 0 & 0 ; \\ 0 & 1 & 0 & 2 & 0 & 6 & 3 & 3 & 0 & 0 & 0 & 0 ; \\ 0 & 0 & 0 & 0 & 0 & 3 & 2 & 3 & 2 & 0 & 0 & 0 ; \\ 1 & 2 & 0 & 1 & 1 & 2 & 1 & 0 & 0 & 0 & 0 & 1 ; \\ 0 & 2 & 0 & 0 & 0 & 4 & 2 & 2 & 2 & 0 & 0 & 0 ; \\ 0 & 0 & 0 & 0 & 0 & 3 & 1 & 2 & 0 & 2 & 0 & 0 ; \\ 0 & 4 & 0 & 3 & 0 & 2 & 3 & 4 & 0 & 2 & 0 & 0 ; \\ 0 & 0 & 1 & 0 & 1 & 2 & 2 & 2 & 0 & 0 & 0 & 3 ; \\ 0 & 0 & 0 & 0 & 0 & 0 & 2 & 0 & 0 & 0 & 1 & 0 ; \\ 0 & 1 & 1 & 4 & 1 & 4 & 1 & 1 & 1 & 2 & 4 & 1]\end{array}$

$\mathrm{nc}=[0 ; 0 ; 0 ; 0 ; 8 ; 1 ; 0 ; 0 ; 3 ; 2 ; 1 ; 0 ; 1 ; 1 ; 0 ; 1 ; 1 ; 1 ; 1 ; 1 ; 1 ; 1]$

nd $=[0 ; 0 ; 0 ; 0 ; 8 ; 1 ; 1 ; 0 ; 3 ; 2 ; 1 ; 0 ; 1 ; 1 ; 0 ; 1 ; 1 ; 1 ; 1 ; 1 ; 0 ; 1]$

$\mathrm{nf}=$

$\left[\begin{array}{llllllllllll}1 & 0 & 0 & 0 & 0 & 0 & 0 & 0 & 0 & 0 & 0 & 0 ;\end{array}\right.$

$\begin{array}{llllllllllll}0 & 1 & 0 & 0 & 0 & 0 & 0 & 0 & 0 & 0 & 0 & 0 ;\end{array}$

$\begin{array}{llllllllllll}0 & 0 & 1 & 0 & 0 & 0 & 0 & 0 & 0 & 0 & 0 & 0 ;\end{array}$

$\begin{array}{llllllllllll}0 & 0 & 0 & 1 & 0 & 0 & 0 & 0 & 0 & 0 & 0 & 0 ; ; ~\end{array}$

$\begin{array}{llllllllllll}0 & 1 & 5 & 0 & 2 & 5 & 2 & 0 & 0 & 0 & 5 & 3 ;\end{array}$

$\begin{array}{llllllllllll}0 & 2 & 2 & 2 & 2 & 0 & 2 & 0 & 0 & 0 & 2 & 2 ;\end{array}$

$\begin{array}{llllllllllll}1 & 1 & 0 & 1 & 1 & 3 & 1 & 0 & 0 & 0 & 0 & 0 ;\end{array}$

$\begin{array}{llllllllllll}2 & 2 & 0 & 1 & 0 & 1 & 3 & 0 & 0 & 0 & 0 & 0 ;\end{array}$

$\begin{array}{llllllllllll}0 & 2 & 0 & 0 & 0 & 2 & 1 & 0 & 0 & 0 & 0 & 0 ;\end{array}$

$\begin{array}{llllllllllll}0 & 1 & 0 & 1 & 0 & 1 & 1 & 0 & 0 & 0 & 0 & 0 ;\end{array}$

$\begin{array}{llllllllllll}0 & 1 & 0 & 0 & 0 & 4 & 1 & 1 & 0 & 0 & 0 & 2 ;\end{array}$

$\begin{array}{llllllllllll}0 & 0 & 0 & 0 & 0 & 0 & 0 & 1 & 0 & 0 & 0 & 0 ;\end{array}$ 


$\begin{array}{llllllllllll}0 & 1 & 0 & 2 & 0 & 2 & 1 & 0 & 0 & 0 & 0 & 0 ; \\ 0 & 1 & 0 & 3 & 0 & 3 & 4 & 3 & 0 & 0 & 0 & 0 ; \\ 0 & 0 & 0 & 0 & 0 & 2 & 2 & 3 & 3 & 0 & 0 & 0 ; \\ 1 & 2 & 0 & 1 & 1 & 3 & 1 & 0 & 0 & 0 & 0 & 1 ; \\ 0 & 2 & 0 & 0 & 0 & 3 & 2 & 2 & 2 & 0 & 0 & 0 ; \\ 0 & 0 & 0 & 0 & 0 & 3 & 3 & 2 & 0 & 3 & 0 & 0 ; \\ 0 & 3 & 0 & 2 & 0 & 2 & 2 & 3 & 0 & 2 & 0 & 0 ; \\ 0 & 0 & 1 & 0 & 1 & 2 & 2 & 2 & 0 & 0 & 0 & 3 ; \\ 0 & 0 & 0 & 0 & 0 & 0 & 2 & 0 & 0 & 0 & 1 & 0 ; \\ 0 & 1 & 4 & 4 & 1 & 4 & 1 & 1 & 4 & 4 & 4 & 1]\end{array}$
$\mathrm{nk}=$

$\begin{array}{llllllllllll}{[1} & 1 & 1 & 1 & 1 & 1 & 1 & 1 & 1 & 1 & 1 & 1 ; \\ 1 & 1 & 1 & 1 & 1 & 1 & 1 & 1 & 1 & 1 & 1 & 1 ; \\ 1 & 1 & 1 & 1 & 1 & 1 & 1 & 1 & 1 & 1 & 1 & 1 ; \\ 1 & 1 & 1 & 1 & 1 & 1 & 1 & 1 & 1 & 1 & 1 & 1 ; \\ 1 & 1 & 1 & 1 & 1 & 2 & 0 & 1 & 1 & 1 & 1 & 1 ; \\ 1 & 1 & 1 & 1 & 1 & 1 & 1 & 1 & 1 & 1 & 1 & 1 ; \\ 1 & 1 & 1 & 1 & 1 & 1 & 1 & 1 & 1 & 1 & 1 & 1 ; \\ 1 & 1 & 1 & 17 & 1 & 1 & 1 & 1 & 1 & 1 & 1 & 1 ; \\ 1 & 1 & 1 & 1 & 1 & 1 & 1 & 1 & 1 & 1 & 1 & 1 ; \\ 1 & 1 & 1 & 1 & 1 & 0 & 1 & 1 & 1 & 1 & 1 & 1 ; \\ 1 & 1 & 1 & 1 & 1 & 1 & 1 & 1 & 1 & 1 & 1 & 1 ; \\ 1 & 1 & 1 & 1 & 1 & 1 & 1 & 1 & 1 & 1 & 1 & 1 ; \\ 1 & 1 & 1 & 3 & 1 & 1 & 1 & 1 & 1 & 1 & 1 & 1 ; \\ 1 & 1 & 1 & 26 & 1 & 1 & 1 & 1 & 1 & 1 & 1 & 1 ; \\ 1 & 1 & 1 & 1 & 1 & 1 & 1 & 1 & 1 & 1 & 1 & 1 ; \\ 1 & 1 & 1 & 1 & 1 & 1 & 1 & 1 & 1 & 1 & 1 & 1 ; \\ 1 & 1 & 1 & 1 & 1 & 0 & 1 & 1 & 1 & 1 & 1 & 1 ; \\ 1 & 1 & 1 & 1 & 1 & 1 & 6 & 22 & 1 & 1 & 1 & 1 ; \\ 1 & 0 & 1 & 0 & 1 & 1 & 0 & 0 & 1 & 1 & 1 & 1 ; \\ 1 & 1 & 1 & 1 & 1 & 1 & 1 & 1 & 1 & 1 & 1 & 1 ; \\ 1 & 1 & 1 & 1 & 1 & 1 & 1 & 1 & 1 & 1 & 1 & 1 ; \\ 1 & 1 & 1 & 1 & 1 & 1 & 1 & 1 & 1 & 1 & 1 & 1] \\ 1\end{array}$




\section{Index}

\section{B}

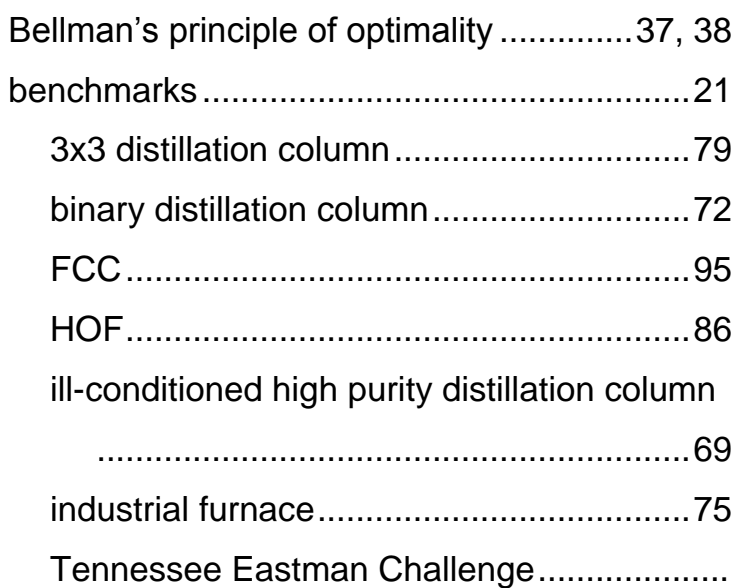

$.22,106,178$

Wood and Berry distillation column .65

\section{C}

control objectives 2,23

controlled variables .27

\section{D}

degrees of freedom

\section{M}

model representations 30

multi-objective optimization augmented lexicographic multi-objective

optimization 45,46

composite function methods . .43

definition .42

goal programming . .44

lexicographic multi-objective optimization. 45

\section{$N$}

Nash equilibrium.

\section{0}

optimal control tuning 50 optimality

robustness .35

sensitivity. .35

$\boldsymbol{P}$
Pareto
front $\ldots \ldots \ldots \ldots \ldots$
optimality
set

Augmented Hierarchical Plantwide Control

Design Technique 56,153

benchmarks 21

definition $4,9,55,155$

design techniques $12,13,14,20$

hybrid techniques 12

literature. 3

mathematical and optimization-based techniques 12

Optimal Multi-Objective Technique for Integrated Control Structure Selection and Controller Tuning 60,154 process-oriented techniques ..................... 12 reviews 9 process modeling 29

\section{$\boldsymbol{R}$}

recycling systems 28

RGA 25

$S$

self-optimizing control 25

snowball effect 28,37

steady-state 25

system identification .................................... 33

procedure 171

$U$

unstable units. 29 